\title{
Erweiterte wissenssoziologische Gattungsanalyse
}

Die erweiterte Gattungsanalyse, welche für die Auswertung der vielfältigen erhobenen Daten herangezogen wurde, ist das Resultat der engen Verzahnung von Theorie- und Methodenarbeit. Mit Luckmann (1986) werden kommunikative Gattungen als verfestigte Formen der Vermittlung sozialer Wirklichkeit verstanden (ebd.: 196). Es handelt sich dabei um „historisch und kulturell spezifische, gesellschaftlich verfestigte und formalisierte Lösungen kommunikativer Probleme“ (Knoblauch \& Schnettler 2010: 4). Die Gattungsanalyse bildet den methodischen Weg, um die Elemente eben dieser Muster herauszuarbeiten. Ziel der nachfolgenden Ergebnisdarstellung der Analyse ist nicht die Etablierung des Laborexperiments als eine kommunikative Gattung - die gattungsförmige Ordnungshaftigkeit des sozialen Phänomens wird vielmehr als gegeben angenommen. Die vorgenommene Anwendung der Gattungsanalyse zielt darauf ab, eben diesen Vermittlungsprozess als den empirisch beobachtbaren Prozess der Rahmenbildung im Labor zu erfassen. Die Gattungsanalyse wurde zu diesem Zwecke als ein wissenssoziologisch motiviertes Verfahren der Daten- und Methodentriangulation genutzt. Dieses erlaubt es, die unterschiedlichen Datenerhebungsmethoden konsistent und gegenstandsadäquat in die Analyse einzubinden. Zugleich erweist sich dieses Verfahren in seiner hier genutzten Form als anschlussfähig an die konzeptionelle Ausrichtung der eigenen Forschungsperspektive, deren Entwicklung im Kapitel 3 dargestellt wurde.

Ein zentrales Moment meiner konzeptionellen Perspektive besteht darin, dass der Vollzug von Interaktionssituationen den Brennpunkt des analytischen Interesses bildet. Die soziale Ordnung wird nicht als etwas Feststehendes betrachtet, sondern als ein Resultat ihrer (Re-)Produktion in empirisch beobachtbaren Handlungen. Soziale Wirklichkeit ist ein gemeinsames Produkt der Handelnden. Dies gilt somit gleichsam für den Prozess der Rahmenbildung im ökonomischen Laboratop, der Gegenstand dieser Arbeit ist. In empirischen Situationen 
tritt Wissen dabei stets als prozessuales Wissen in Erscheinung und entäußert sich in seiner Anwendung in konkreten Handlungssituationen. Eine kommunikative Gattung wird von mir als eine musterhafte und typische Form der Entäußerung von Wissenselementen verstanden, auf welche die Handelnden in ihrem Tun Bezug nehmen. Die drei Ebenen der Gattungsanalyse fokussieren dabei unterschiedliche Arten dieser Wissenselemente und musterhafte Formen ihrer Entäußerung. Die Differenzierung in die drei Ebenen der Außenstruktur, Binnenstruktur und situativen Realisierungsebene, welche die Gattungsanalyse vornimmt, ist eine analytische Trennung. Diese verfolgt das Ziel, die Strukturmerkmale von kommunikativen Gattungen entsprechend ihrer unterschiedlichen ,Qualitäten' voneinander zu differenzieren. In der empirischen Wirklichkeit der Handelnden wirken die drei Ebenen der Gattung jedoch gleichzeitig und sind miteinander verwoben.

Um dies zu verdeutlichen, erfolgt in einem ersten Schritt dieses Kapitels (Abschnitt 5.1) die exemplarische Darstellung der Analyse einer Anfangssequenz eines Experiments. Für diesen Zwischenschritt, der vor der eigentlichen Ergebnisdarstellung getätigt wird, gibt es zwei Gründe. Einerseits wird so die Verwobenheit der Gattungsmerkmale in der empirischen Situation illustriert und andererseits wird hier beispielhaft das analytische Vorgehen dargestellt, welches in dieser Arbeit Anwendung findet.

Die daran anschließenden drei Unterkapitel (5.2, 5.3, 5.4) widmen sich den Ergebnissen des Analyseprozesses. Sie zielen entsprechend nicht darauf $\mathrm{ab}$, die Analyse selbst darzustellen, sondern sind auf die Beantwortung der Forschungsfrage ausgerichtet. Sie stellen die musterhaften Strukturmerkmale in systematisierter Form dar, die den Rahmenbildungsprozess im Vollzug der wirtschaftswissenschaftlichen Laborexperimente charakterisieren. Hier wurde die Analyseheuristik der Ebenendifferenzierung aus der Gattungsanalyse übernommen, um die unterschiedlichen Merkmale des Untersuchungsgegenstandes zu separieren und dem oben skizzierten eigenen Anspruch der gegenstandsadäquaten Integration der unterschiedlichen Datensorten zu genügen.

Die Außenstruktur (Abschnitt 5.2) umfasst solche rahmungsrelevanten Aspekte, die als Kontextelemente der sozialen Veranstaltung des wirtschaftswissenschaftlichen Experiments eigen sind. Es handelt sich hier um rahmungsrelevante Elemente, die den Kontext der empirischen Situation erweitern und auf Wissensbestände Bezug nehmen, die außerhalb der beobachteten Situation liegen. Es wird hier beispielsweise auf das Set der sozialen Rollen und das soziale Milieu eingegangen, welche wirtschaftswissenschaftlichen Experimenten zugehörig sind. Zudem werden paradigmatische Annahmen der experimentellen WirtschaftswissenschaftlerInnen expliziert, welche sich in der Durchführung und Konzeption 
ihrer Experimente als handlungsleitend erweisen. Die Ebene der Außenstruktur bildet dabei eine zentrale Grundlage für die Entschlüsselung typischer Versuche und Strategien der Rahmenbildung und -verankerung, welche sich in der Durchführung von wirtschaftswissenschaftlichen Laborexperimenten entäußern.

Die Ebene der Binnenstruktur und der situativen Realisierungsebene umfasst musterhafte Merkmale der Rahmenbildung, die den empirischen Vollzug des wirtschaftswissenschaftlichen Experimentierens im Labor prägen. Es handelt sich dabei um musterhafte Formen kommunikativen Handelns, die als Ethnomethoden der handelnden Akteure, technische oder materielle Objektivationen in Erscheinung treten. Die Ebene der Binnenstruktur, welche Gegenstand des Abschnitts 5.3 ist, thematisiert Merkmale des beobachtbaren Rahmenbildungsprozesses, die vom situativen Vollzug unabhängig sind. Der Abschnitt 5.4 widmet sich den Merkmalen des Rahmenbildungsprozesses, welche der Ebene der situativen Realisierung zugeordnet werden können. Bei diesen handelt es sich um eben solche typischen Merkmale des Rahmenbildungsprozesses, die sich der interaktiven Handlungskoordination der Laborsituation zuordnen lassen. Die Merkmale der Ebene der situativen Realisierung und der Binnenstruktur bezeichnen musterhafte Formen kommunikativen Handelns, welche für den Prozess der Rahmenbildung im Labor charakteristisch sind. Um den spezifischen Beitrag dieser einzelnen Merkmale genauer zu erfassen, wurde eine Analyseheuristik entwickelt, die zwischen vier unterschiedlichen Ebenen des „Doing“ hinsichtlich ihres Beitrags zur Rahmenbildung differenziert. ${ }^{1}$ Auf diese Weise wird deutlich, welche Rahmungs- bzw. Modulationseigenschaften diese Merkmale für die Herausbildung des ökonomischen Laboratops besitzen.

Der fünfte Teil dieses Kapitels (5.5) widmet der Anwendung des im Abschnitt 4.1.3 eingeführten qualitativen Experiments. Die Anwendung des qualitativen Experiments zielt hier darauf ab, ausgewählte Ergebnisse des eigenen Forschungsprozesses einer Überprüfung zu unterziehen. Es handelt sich somit einerseits um einen Validitätstest ausgewählter Forschungsergebnisse. Darüber hinaus zielt die Darstellung darauf ab, den Wert dieses qualitativen und zugleich experimentellen Verfahrens anhand seiner Anwendung zu illustrieren.

Abschließend fasst Abschnitt 5.6 die Ergebnisse des Forschungsprozesses und der Analyse zusammen. In diesem Rahmen werden in einem ersten Schritt die konzeptionellen und methodologischen Erkenntnisse des Forschungsprozesses dargelegt (Abschnitt 5.6.1). Anschließend wird durch die systematische Zusammenfassung der inhaltlichen Ergebnisse (Abschnitt 5.6.2) eine resümierende Beantwortung der Forschungsfrage vollzogen.

\footnotetext{
${ }^{1}$ Die Analyseheuristik wurde zu Beginn des Abschnitt 5.3 eingeführt.
} 


\subsection{Exemplarische Darstellung der Verwobenheit der Gattungsebenen}

In einem ersten Schritt wird nun das, an der Videographie orientierte, analytische Vorgehen dargestellt, welches in dieser Arbeit einen zentralen Bezugspunkt der Analyse bildete. Der im Folgenden betrachtete Datenausschnitt stellt die Anfangssequenz eines Laborexperiments dar. Dieser wurde mittels einer Deckenkamera im Labor aufgezeichnet. In einem ersten Schritt wird ein Basistranskript (I) der verbalen Kommunikation präsentiert. Anschließend werden die beobachtbaren Handlungsvollzüge der Interaktionssituation analysiert (II).

Der Inhalt des Datenausschnitts teilt sich dabei in drei Handlungskomplexe:

(A) Die Abstimmung der ExperimentatorInnen über die Beendigung der Vorbereitungen und den Auftakt des Einlasses.

(B) Den Einlass der Teilnehmenden und das Suchen und Einnehmen ihrer Arbeitsplätze.

(C) Die Begrüßung und Belehrung durch das Laborteam.

Diese drei Abschnitte (A, B, C) werden zur besseren Leseorientierung sowohl im nachfolgenden Basistranskript als auch in der Analyse der Interaktionssituation ausgewiesen. Wie in der Einleitung des Hauptkapitels bereits erwähnt, zielt dieses Abschnitt 5.1 darauf ab, die kumulierte analytische Gesamtsituation beispielhaft aufzuzeigen. Die dichte Darstellung der empirischen Situation dient dabei dazu, exemplarisch die Verwobenheit der Gattungsebenen in der empirischen Situation aufzuzeigen. Dies erfolgt, indem im Zuge der sich vollziehenden Handlungssequenzen an den jeweiligen Stellen einzelne rahmungsrelevante Aspekte in Unterabschnitten thematisiert werden. Bei diesem Vorgehen kommt es unweigerlich zu Redundanzen mit den Aspekten der nachfolgenden Darstellung der Ergebnisse der Gattungsanalyse. Diese besitzen jedoch einen anderen Erklärwert, da nicht ihre sequenzielle Positionierung von Interesse ist, sondern ihr spezifischer Beitrag zum Prozess der Rahmenbildung und -verankerung.

\section{(I) Basistranskript}

Im Transkript wurden die Personennamen anonymisiert. Die Transkription erfolgte in gemischter Transkriptionsschreibweise in Anlehnung an Kruse (2008). Es wurde alles kleingeschrieben, um bei Bedarf durch Großschreibung auf eine verstärkte Betonung von Wörtern oder Wortteilen hinzuweisen.

In der betrachteten Kommunikationssituation treten folgende Handelnde auf: 
Juliane (Experimentatorin), Tim (Experimentator), Silke (Probandin 1), Ina (Probandin 2), Jana (Probandin 3), Unid1, Unid2, Unid3 (Unid = unidentifizierte Person vor dem Labor).

\section{(A) Die Abstimmung der ExperimentatorInnen}

1 Juliane:können wir?

2 Tim: ja (.) Super

(B) Einlass und Platzsuche der ProbandInnen, Einnehmen der Arbeitsplätze

3 Tim: dann (.) ähm (1.0)

4 bitte eintreten und die einverständniserklärungen mir geben

5 Unid1: und an die nummer setzen?

6 Tim: GENAU, AUF DEN PLATZ SETZEN (.) MIT DER NUMMER AUF DEM CHIP

7 Unid2: (...)

8 Tim: ${ }^{\circ}>$ stehen an der wand $<{ }^{\circ}$

9 Jana: du bist hier ((wendet sich zu Ina und macht eine Zeigegeste))

10 ach Gott und ich bin hier

11 das ist ja auch was ((lacht))

12 Unid3: ((unverständliches Gemurmel vor dem Labor))

13 Tim: die anwesenheitsliste ist jetzt voll.

14 bitte füll das noch aus

15 danach komm ich gleich raus.

16 Tim: (...) ((macht mit der linken Hand eine Zeigegeste in Julianes Richtung))

(C) Begrüßung und Belehrung durch das Laborteam

17 Juliane:SO: herzlich willkommen zu dem experiment.

18 wenn ihr ((räuspern)) euch jetzt alle eingefunden habt, dann kann es ja jetzt losgehen.

19 ihr habt ja auf eurem platz alle ein paar instruktionen zu liegen

20 und wir werden auch gleich damit starten.

21 kurz noch zu den ALLgemeinen regeln (.)

22 grundsätzlich ist es so, dass ihr bitte nicht miteinander kommunizieren sollt.

23 jeder macht das sozusagen für sich allein (.)

24 ähm des weiteren sollen bitte keine elektronischen geräte benutzt werden 
25 und sollte es fragen geben unklarheiten $>$ wie auch immer<

26 dann gebt mir bitte ein zeichen oder uns

27 >damit wir dann zwischendurch nicht so einen zeitdruck haben<

28 und dann kommen wir einzeln zu euch und beantworten die fragen.

29 sollte die frage so wichtig sein, dass sie für alle geklärt werden muss, dann stellen wir

30 sie noch einmal laut oder beantworten sie noch mal laut

31 und dann lassen wir alle anderen das auch wissen.

32 ansonsten könnt ihr jetzt bitte starten die instruktionen zu lesen und wenn wir dann

33 fertig sind, dann beginnt das experiment.

34 danke

\section{Transkriptionskonventionen:}

\begin{tabular}{l|l}
\hline \multicolumn{1}{c|}{$=$ Emphase } &,$=$ leicht steigende Intonation \\
\hline$(1.0)=1$-sekündige Pause &.$=$ fallende Intonation \\
\hline$((\ldots))=$ Analysekommentar & {$[=$ Überlappung } \\
\hline$()=$. kurze Pause & $>\ldots<=$ schneller \\
\hline$?=$ steigende Intonation & ${ }^{\circ}=$ ruhiger \\
\hline$(\ldots)=$ unverständlich & $\mathrm{UND}=$ lauter \\
\hline$:=$ Dehnung & \\
\hline
\end{tabular}

\section{(II) Die Analyse der Handlungsabläufe}

Während das Basistranskript (I) die Abfolge der verbalsprachlichen Redezüge fokussiert, widmet sich die folgende Analyse den im Experimentallabor beobachtbaren Handlungsabläufen. Die Videosequenz, die hier als „Eingangssequenz“ bezeichnet wird, lässt sich dabei in weitere kleine Handlungssequenzen unterteilen. Auftakt und Ende dieser Handlungssequenzen werden dabei durch die kommunikativen Handlungen der Akteure selbst definiert und angezeigt und sind auf diese Weise auch im Datenmaterial ersichtlich. Die Darstellung der einzelnen Handlungssequenzen wird dabei jeweils in einen deskriptiven und einen interpretativen Teil getrennt. Die Deskription zum Zwecke der Videographie erhebt dabei nicht den Anspruch, alle im Videomaterial ersichtlichen Aspekten zu beschreiben. Sie ist vielmehr an der detaillierten Explikation und der prozesshaften Abfolge der analytisch relevanten Aspekte interessiert (Wilke \& Lettkemann 2018). Eingeleitet wird jede betrachtete Handlungssequenz durch zwei bis drei Fotos, welche die analytisch zentralen Momente illustrieren. Im Fall der Vergrößerung einzelner Bildausschnitte stellt ein zweites Bild dabei jeweils die Totale dar. 
Im zweiten Teil der jeweiligen Darstellung wird die Aufmerksamkeit auf exemplarisch ausgewählte Aspekte gelenkt, die in der späteren Analyse noch detaillierter betrachtet werden. Es handelt sich dabei um charakteristische Merkmale der Rahmenbildung des wirtschaftswissenschaftlichen Laboratops, auf welche in der nachfolgenden Gattungsanalyse Bezug genommen wird. Unter Hinzunahme ethnographischen Wissens wird auf handlungsrelevante Details der Situation verwiesen, die sich einer einfachen Beobachtung aus der Kameraperspektive entziehen.

\section{(A) Abstimmung über die Abgeschlossene Vorbereitung}

Zur Etablierung der Analysesituation wird in einem ersten Schritt die materielle Struktur des Laborraums beschrieben, in welchem sich die nachfolgenden Handlungssequenzen abspielen.

\section{Das materielle Setting des Laborraum}

Abb. 5.1a Laborraum (Totale)

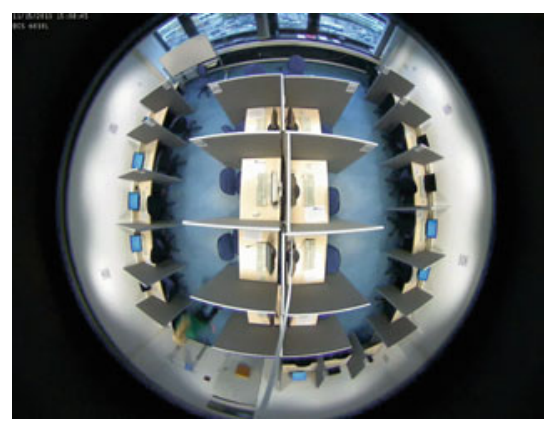

Abb. 5.1b Laborraum (Zoom)

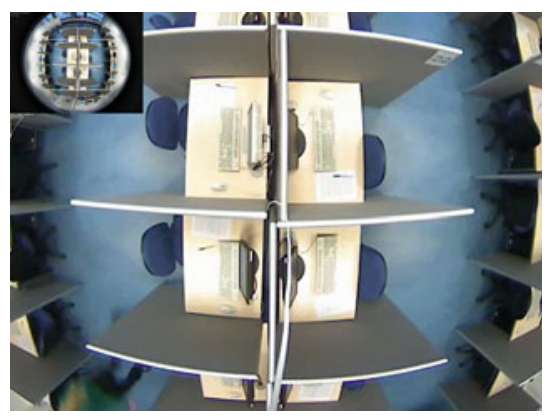


Die Eingangssituation der analysierten Sequenz zeigt ein Experimentallabor mit unbesetzten Arbeitsplätzen. Im Laborraum befindet sich nur eine Person mit grünem Oberteil und schwarzer Hose. Da den materiellen Aspekten der Handlungssituation im Rahmen dieser Arbeit für die kommunikative Konstruktion eine große Bedeutung beigemessen wird, wird der Laborraum mit seiner speziellen räumlichen Struktur und Ausstattung im Folgenden einführend beschrieben.

Abbildung 5.1a und 5.1b vermitteln einen Eindruck des Laborraums. Die Wände des Raums sind weiß gestrichen, sein Fußboden ist blau. An der unteren Wand des Raums befindet sich eine Tür, an der eine braune Kiste befestigt ist. An der gegenüberliegenden Seite des Raums ist eine große Fensterfront zu sehen, vor der einige Unterlagen auf dem Fensterbrett abgelegt sind.

Betrachtet man diese Abbildungen genauer, so fällt ins Auge, dass durch die Nutzung grauer Trennwände eine weitere Unterteilung des Raums realisiert wurde. Spezifischer wurden im Laborraum durch den Einsatz von 30 grauen Trennwänden 25 einzelne Kabinen geschaffen. Es handelt sich bei diesen Kabinen um die Arbeitsplätze für die Versuchsteilnehmer. Diese sind jeweils von drei Seiten geschlossen und können somit nur von einer Seite aus betreten werden.

An der linken sowie an der rechten Wand des Raumes befinden sich jeweils sieben Kabinen und an der unteren Seite des Raumes, rechts neben der Tür, drei weitere Arbeitsplätze. In der Mitte des Raumes sind in einer Zweierreihe acht weitere Arbeitsplätze eingerichtet. Zwischen den an der Wand positionierten Arbeitsplätzen und jenen in der Mitte verläuft ein Gang, der es ProbandInnen und ExperimentatorInnen ermöglicht, die Arbeitsplätze zu erreichen. Die einzelnen Kabinen sind mit Zahlen von 1 bis 25 versehen. An der linken Wandseite des Raums befinden sich die Kabinen mit den Nummer 1 bis 7, auf der gegenüberliegenden rechten Wandseite diejenigen mit den Nummern 8 bis 14. Im mittleren Achterblock sind links die Kabinen 17 bis 20 und rechts diejenigen mit der Nummer 21 bis 24 angeordnet. An der unteren Wandseite, rechts neben der Tür, sind die Kabinen 15, 16 und 25 positioniert. Die Beschriftung mit Arbeitsplatznummern ist dabei stets so angebracht, dass sie aus der Laufrichtung von der Eingangstür zur Fensterfront eingesehen werden kann.

Die 25 Kabinen sind jeweils gleichartig ausgestattet (siehe Abb. 5.1b). Sie verfügen über einen Tisch mit einer hellen Arbeitsplatte, der in allen Kabinen jeweils die gleiche Größe besitzt, ${ }^{2}$ sowie einen blauen Drehstuhl und einen Desktopcomputer. Auf der Tischplatte befinden sich in allen Kabinen ein Computerbildschirm,

\footnotetext{
${ }^{2}$ Dass die Arbeitsplätze in der Mitte auf dem Bild größer erscheinen, ist der Kameraperspektive geschuldet. Die Kamera wurde an der Decke des Laborraums über dem mittleren Block von Arbeitsplätzen befestigt und verfügte über ein Fischaugenobjektiv, welches in der Lage ist, in einem $360^{\circ}$ Winkel den gesamten Raum zu filmen. Mit diesem Vorteil gehen jedoch eine
} 
eine Tastatur und eine Computermaus. Zur weiteren Ausstattung der Laborarbeitsplätze gehören vor Beginn eines Experiments ein Stift und einige Seiten bedrucktes Papier. Es handelt sich bei den ausliegenden Unterlagen um die spezifischen Instruktionen für das jeweilige Experiment. Einen weiteren Aspekt der Laborausstattung stellt ein mobiles Whiteboard mit einem rollbaren Gestell dar, welches sich in der linken oberen Ecke des Raumes befindet. In der linken unteren Ecke stehen zudem ein Papierkorb und ein Kleiderständer. Im Rahmen des Experiments, dem der analysierte Datenausschnitt entstammt, wurden an der rechten und linken Wand des Raumes jeweils zwei Hinweisschilder angebracht und ein weiteres an der Tür des Laborraums. Diese zeigten den Schriftzug „Achtung, Videoüberwachung!“ sowie die Abbildung einer Videokamera. ${ }^{3}$ An der oberen Seite des mittigen Achterblocks von Kabinen, nahe der Fensterfront, befindet sich ein weiterer Sitzbereich. Aus der Kameraperspektive sind lediglich zwei blaue Stühle zu erkennen.

Aufgrund der Relevanz für das Setting des Labors und für das Verständnis der nachfolgenden Handlungssequenzen werden nun - exemplarisch für weitere materielle Aspekte - den Ausführungen zu dem Sitzbereich an der Fensterfront weitere Details hinzugefügt. Diese beruhen auf teilnehmenden Beobachtungen des Laborgeschehens und auf dem Kontextwissen über die Forschung im Labor.

Die beiden gerade thematisierten blauen Stühle vor der Fensterfront stehen an einem großen Tisch, welcher mit zwei Computerbildschirmen und Desktopcomputern ausgestattet ist. Es handelt sich dabei um den Sitzbereich der ExperimentatorInnen. Dieser Ort nimmt in der Koordination und Überwachung des Handlungsgeschehens im Labor eine zentrale Stellung ein. Wie die späteren Ausführungen zu den Gliederungsmerkmalen des Laborexperiments (Abschnitt 5.3.1) zeigen werden, umfasst das Phänomen eine Abfolge typischer Phasen. Die einzelnen Programmabschnitte und Dateien, die den Phasen der Experimente entsprechen, werden dabei vom Arbeitsplatz der ExperimentatorInnen aus gestartet. Der Übergang zwischen den einzelnen Phasen wird dabei durch eine spezifische Verbindung von verbalen Äußerungen und technischen Handlungen realisiert. In den von mir beobachteten Experimenten, wie auch in vielen anderen Laboren, wird dafür bevorzugt auf ein spezielles Softwareprogramm namens z-Tree (Fischbacher 2007) zurückgegriffen. Dieses zielt unter anderem darauf ab, eine Standardisierung der Kommunikation mit den ProbandInnen zu erleichtern, indem

gewisse Verzerrung der Größenverhältnisse sowie eine Krümmung der gefilmten Aspekte in den Randbereichen der Aufnahme einher.

${ }^{3}$ Die Hinweisschilder sind nicht Teil der typischen Ausstattung des Labors, sondern wurden aufgrund meiner Datenerhebung angebracht. 
allen ProbandInnen gleichartige Informationen zur Verfügung gestellt werden und diese in gleicher Weise auf ihren Computerbildschirmen abgebildet werden. Wie in späteren Ausführungen zum Aspekt der Mediation des kommunikativen Handlungsgeschehens (Abschnitt 5.3.8) noch detaillierter dargestellt wird, werden die einzelnen Entscheidungssituationen und Informationen den Teilnehmenden am Computerbildschirm angezeigt. Die Auswahlmöglichkeiten oder Eingaben, die die ProbandInnen tätigen können, sind dabei maßgeblich durch die programmierte Oberfläche der Bildschirmanzeigen vorstrukturiert. Die Bedeutung, die der Arbeitsplatz der ExperimentatorInnen für den Auftakt des Experiments besitzt, besteht darin, dass die dort stattfindende technische Koordination der zu besetzenden Arbeitsplätze vorbereitet werden muss. Es werden hier bereits vor dem Einlass der ProbandInnen, die im Folgenden mit ProbandInnen zu besetzenden Kabinen ausgewählt und dort die benötigten Computerprogramme gestartet. Dies ist erforderlich, da in vielen Experimenten nicht alle 25 Kabinen des Labors besetzt werden. Ein häufiger Grund dafür ist, dass die Teilnehmenden im Rahmen der Experimente in Gruppen zusammenspielen oder es eine angestrebte Stichprobengröße gibt. Auch in dem Experiment, dessen Anfangssequenz hier betrachtet wird, wurden nur 20 der 25 Arbeitsplätze besetzt. Aus diesem Grund war es auch hier notwendig, die Auswahl der Arbeitsplätze zu koordinieren, bevor die ProbandInnen das Labor betraten.

\section{Der Auftakt -Abstimmung über abgeschlossene Vorbereitungen}

Abb. 5.2a Auftakt (Totale)

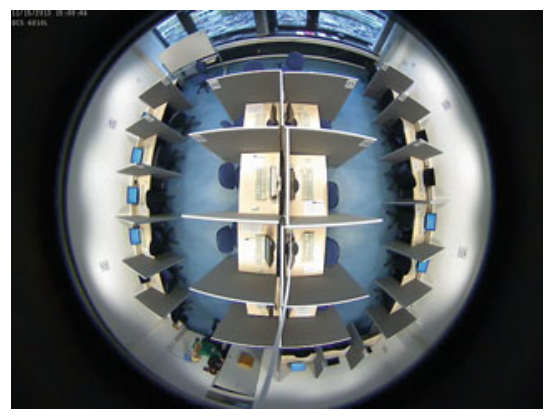


Abb. 5.2b Auftakt (Zoom)

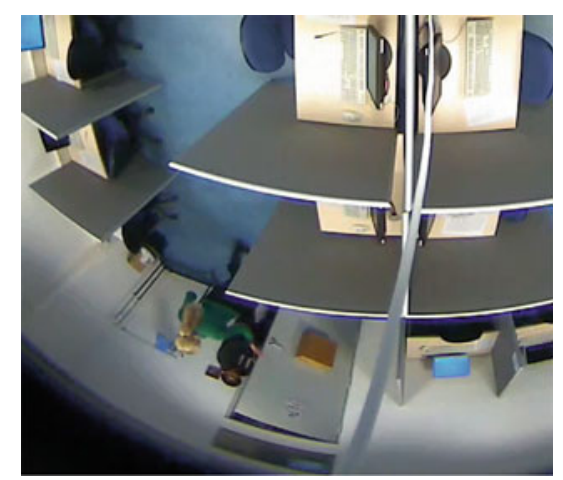

$\mathrm{Zu}$ Beginn der analysierten Videosequenz befindet sich die Experimentatorin Juliane allein im eingangs beschriebenen Laborraum (Abb. 5.2a.). Schnellen Schrittes läuft sie den linken Gang des Raumes entlang Richtung Tür. Die Tür ist bereits einen Spalt breit geöffnet. Noch bevor sie an der Tür angekommen ist, streckt sie ihre linke Hand in Richtung der Tür aus. Zeitgleich mit ihrem Erreichen der Tür wird diese von außen durch den Experimentator Tim ein kleines Stück geöffnet. Julianes Hand verharrt an der Türkante und verhindert damit, dass sich die Tür weiter öffnet. Der Experimentator Tim bleibt in der Tür stehen und schaut zu Juliane (Abb. 5.2a/5.2b). Diese blickt ihn wiederum an und eröffnet die Konversation mit der Frage:

Z 1: Juliane: können wir?

Durch diese Frage wird Tim als nächster Sprecher adressiert. Bezugnehmend auf Julianes Frage antwortet Tim kurz:

\section{Z 2: Tim: ja (.) Super}

Während er dies sagt, wendet er seinen Blick bereits von Juliane ab und dreht sich seitlich von ihr weg. Er verlässt das Labor und tritt wieder vor die Tür, welche halbgeöffnet bleibt. Juliane schaut Tim noch kurz nach, wendet sich dann aber auch ab und geht mit schnellen Schritten wieder den Gang entlang Richtung Fensterfront.

Die handlungskoordinierende Funktion dieses kurzen Redezugswechsels wird erst durch die Bezugnahme auf das Kontextwissen über die Situation des Experiments verständlich. Die Ankunft der ProbandInnen und ihre Registrierung vor 
Beginn des Experiments ereignen sich vor der geschlossenen Tür des Labors. Es handelt sich hierbei um Aspekte, die vor der fokalen Situation der Analyse liegen, jedoch eine deutliche Relevanz für das Verständnis des Handlungsvollzugs haben. Sie schaffen zudem zentrale Vorbedingungen für den erfolgreichen und regelkonformen Ablauf des Experiments und werden aus diesem Grund im Rahmen der Binnenstruktur als Präsequenz im Zusammenhang mit den Gliederungsmerkmalen (Abschnitt 5.3.1) genauer thematisiert. Im dargestellten Redezugwechsel von Juliane und Tim bestätigen sich die beiden ExperimentatorInnen gegenseitig, dass sie die notwendigen Vorbereitungen für das Experiment abgeschlossen haben und die Teilnehmenden nun das Labor betreten könnten. Juliane überprüft dabei mit ihrer an Tim gerichteten Frage „können wir?“ (Zeile 1), ob dieser die Überprüfung der Personalien der eingeladenen ProbandInnen bereits abgeschlossen hat und eine ausreichende Anzahl an ProbandInnen erschienen ist, um das Experiment wie geplant durchzuführen. Tims bestätigende Antwort ,ja (.) Super“ (Zeile 2) setzt Juliane darüber in Kenntnis, dass diese Handlungsschritte erfüllt sind. Zugleich gibt auch Juliane mit ihrer Frage preis, dass von ihrer Seite aus alle relevanten Vorarbeiten erledigt wurden und somit alle Arbeitsplätze für den Beginn des Experiments vorbereitet und mit den notwendigen Utensilien wie schriftlichen Instruktionen und Stiften ausgestattet wurden. Dass sich beide Akteure voneinander abwenden, signalisiert hier zugleich das Ende der Handlungssequenz.

Da sich die beiden Handelnden in unterschiedlichen Räumen befunden haben und aus diesem Grund eine visuelle Überprüfung der Handlungen des anderen nicht möglich war, nutzen sie hier in typischer Weise eine musterhafte Floskel zur situativen Koordination des weiteren Handlungsgeschehens. Dieser Aspekt stellt ein Merkmal der situativen Realisierungsebene dar, da sich die ExperimentatorInnen hier auf musterhafte Weise darüber abstimmen, ob die notwendigen Vorbereitungen im Labor sowie vor dem Labor abgeschlossen sind. Es handelt sich hierbei somit um ein Merkmal der situativen Handlungskoordination, welches dem Übergang von der Präsequenz der Vorbereitungen zur fokalen Situation des Experiments im Labor dient. 
(B) Einlass und Platzsuche der ProbandInnen, Einnehmen der Arbeitsplätze

Die Regulation des Einlasses und Einverständniserklärungen

Abb. 5.3a Einlass (Totale)

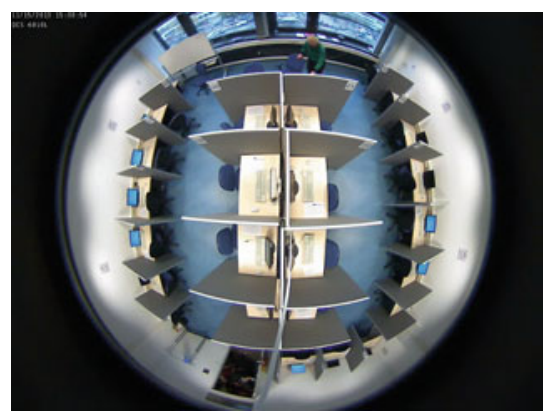

Abb. 5.3b Einlass (Zoom)

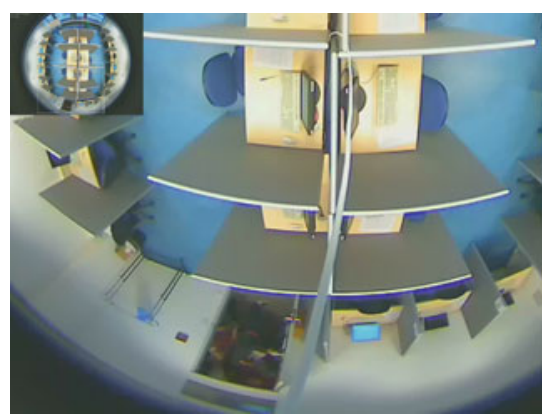

Die Tür schwingt langsam auf und gibt den Blick auf eine Gruppe von Personen frei die sich vor dem Labor befinden (Abb. 5.3a/ 5.3b). Tim ist diesen Personen zugewandt. Er wählt sich selbst als Sprecher und richtet sich mit folgender verbalen Äußerung an die vor dem Labor befindlichen Personen:

Z 3: Tim: dann (.) ähm (1.0)

Z 4: $\quad$ bitte eintreten und die einverständniserklärungen mir geben 
Tim informiert die wartenden ProbandInnen mit seinem Redezug darüber, dass die Phase der Anmeldung und des Wartens vor dem Labor nun beendet und der Zeitpunkt gekommen ist, das Labor zu betreten. Während er die Worte „dann (.) ähm (1.0)“ (Zeile 3) spricht, tritt er in den offenen Türbereich und nimmt eine für die interaktive Koordination des Handlungsgeschehens regulierende Körperposition ein. Sein Rücken ist dabei zur linken Türseite gerichtet und nur wenig Platz verbleibt zwischen diesem und dem Türrahmen. An seiner Vorderseite ist hingegen deutlich mehr Platz. Er signalisiert durch diese Körperposition auf welcher Seite die ProbandInnen an ihm vorbeigehen sollen.

Mit seiner Aussage „bitte eintreten und die Einverständniserklärungen mir geben“" (Zeile 4) verweist er darauf, dass die Teilnehmenden ihm vor Betreten des Labors ihre Einverständniserklärungen aushändigen müssen. Durch seine Körperposition ist Tim in der Lage, visuell zu kontrollieren, dass die eintretenden Personen seiner Handlungsaufforderung nachkommen. Die ProbandInnen verstehen die verbalen und körperlichen Zeichen, die Tim ihnen gibt, und kommen seinen Handlungsaufforderungen in entsprechender Weise nach. Sie schreiten vorn an ihm vorbei und reichen ihm im Vorbeigehen die Einverständniserklärungen.

Die hier adressierte Einverständniserklärung stellt einen Sonderfall dar, der durch die Art meiner Datenerhebung bedingt ist. Wie im Methodenkapitel im Rahmen der Diskussion unterschiedlicher Reaktanzformen (Abschnitt 4.2.2.2) kurz ausgeführt, erachteten die ökonomischen LaborforscherInnen die Erhebung von Videodaten als eine potenzielle Störquelle für nachfolgende Erhebungen. Aus diesen sowie aus datenschutzrechtlichen Gründen wurden die Teilnehmenden bereits im Rahmen der versendeten Einladungen darüber informiert, dass Videoaufnahmen der Experimente angefertigt werden. Zudem mussten sie vor dem Eintreten ins Labor eine Einverständniserklärung für die Videoaufzeichnungen unterschreiben. Wie oben im Zuge der materiellen Ausstattung des Laborraums dargestellt, wurden zusätzlich im Labor fünf Hinweisschilder angebracht, um auf die Videoaufnahme zu verwiesen.

Die teilnehmenden ProbandInnen sind angemeldete Mitglieder eines ProbandInnenpools, welchen die Forschenden nutzen, um passende Forschungssubjekte für ihre Experimente einzuladen. Auf die Zusammensetzung des ProbandInnenpools und die Akquisetechniken, die genutzt werden, um neue ProbandInnen anzuwerben, wird im Zuge der Außenstruktur noch vertiefend eingegangen (siehe Abschnitt 5.2.4). Auch wenn es datenschutzrechtlich und forschungsethisch in jedem Fall notwendig gewesen wäre, auf die Videoaufzeichnung hinzuweisen, ist der Nachdruck, mit dem dies im Analysebeispiel erfolgte, maßgeblich als ein Ausdruck der epistemisch begründeten Bedenken der ökonomischen ForscherInnen zu 
interpretieren. Die Reinhaltung der Laborsituation von Störungen, die die Datenerhebung beeinflussen (könnten), ist - ganz im Sinne des naturwissenschaftlichen Vorbilds aber auch in Anerkennung der Grundlagen der Methodologie sozialwissenschaftlicher Experimente (siehe Abschnitt 2.1) - eine zentrale Prämisse der wirtschaftswissenschaftlichen Laborforschung und Ausdruck der Wirkung der Außenstruktur.

Die potenzielle Gefahr, die in der Erhebung von Videodaten gesehen wurde, bestand darin, dass im untersuchten Labor bisher keine Videokameras eingesetzt wurden und man besorgt war, dass dies nachfolgende Datenerhebungen im Labor auf unberechenbare Weise beeinflussen könnte. So wurde befürchtet, dass teilnehmende ProbandInnen bei nachfolgenden Experimenten im Labor möglicherweise vermuten könnten, dass erneut Videoaufnahmen gemacht würden, ohne dass man sie diesmal informiert hätte, und dass sie so das Vertrauen in die Aufrichtigkeit der Forschenden verlieren könnten. Eine zusätzliche Befürchtung bestand darin, dass die ProbandInnen erwarten könnten, dass sie gefilmt werden und sich anders als gewohnt verhalten würden. Der gesamte Vorlauf, der für die Erhebung von Videodaten im Labor notwendig war, sowie die praktischen Vorkehrungen vor Ort lieferten aus ethnographischer Perspektive zugleich aufschlussreiche Informationen über die disziplinär begründeten Ansichten und Ansprüche der Forschenden hinsichtlich der Laborhygiene. Dass die ForscherInnen besonders darauf bedacht waren, bei den ProbandInnen nicht der Eindruck einer potenziellen Täuschung zu erwecken, bildet zudem ein charakteristisches Moment der Methodologie wirtschaftswissenschaftlicher Experimente. Auf diesen Aspekt wird im Abschnitt über die Außenstruktur unter 5.2.3 noch einmal eingegangen.

\section{Der Spielchip als Koordinationsobjekt}

Abb. 5.4a Platzsuche 1

(Totale)

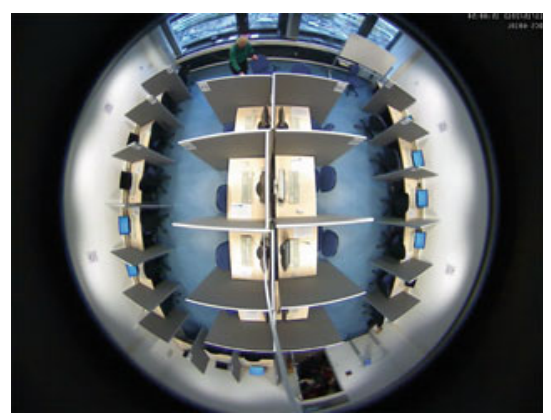


Abb. 5.4b Platzsuche 1 (Zoom)

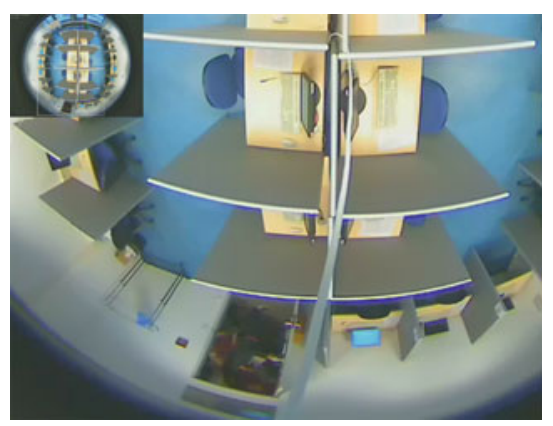

Wie oben dargestellt, hat der Experimentator Tim seinen Körper nun so ausgerichtet, dass er seitlich in der geöffneten Tür steht. Er hält einige Blätter Papier in seiner rechten Hand (Abb. 5.4a/ 5.4b). Mit der linken Hand greift er nach einem Blatt, das ihm von einer Person außerhalb des Sichtbereichs entgegengestreckt wird. Tim nimmt dies entgegen und legt es zuoberst auf seinen Papierstapel. Zeitgleich mit dem Eintreten des ersten Teilnehmenden (P1) vernimmt man die leise Frage eines unidentifizierbaren Sprechers (Unid1), der sich vor dem Labor befindet:

Z 5: Unid1: und an die nummer setzen?

In Reaktion auf diese Frage antwortet Tim laut und für alle Wartenden deutlich hörbar:

Z 6: Tim: GENAU, AUF DEN PLATZ SETZEN (.) MIT DER NUMMER AUF DEM CHIP

Nach einem unverständlichen Redebeitrag eines vor dem Labor befindlichen unidentifizierten Sprechers:

Z 7: Unid2: (...)

Fügt Tim deutlich leiser hinzu:

Z 8: Tim: $\quad{ }^{\circ}>$ stehen an der wand $<{ }^{\circ}$

Der vorangestellte Redezugwechsel thematisiert, auf welche Weise die Platzwahl im Labor realisiert wird. Die Prosodie der Frage legt dabei den Eindruck nahe, 
dass es sich bei der Frage in Zeile 5, ,und an die nummer setzen?“, um eine Suggestivfrage handelt, die eine Behauptung oder Vermutung von Unid1 darstellt.

Diese Frage zeigt somit an, dass Unid1 annimmt, dass es eine Nummer gibt, mittels derer bestimmt wird, an welchen Platz sich die Teilnehmenden setzen sollen. Er zielt in seiner Prosodie eher auf eine Versicherung ab, dass die von Unid1 angenommene Deutung richtig ist. Als Antwort wird aus diesem Grund eine Bestätigung oder Zurückweisung erwartet. Als Reaktion auf diese Frage wählt Tim sich selbst als Sprecher und nimmt in seiner Antwort (Zeile 6) auf die leise gestellte Frage Bezug. Der Beginn seiner Antwort, Zeile 6 „GENAU“, stellt dabei die Bestätigung der Vermutung von Unid1 dar. Tim paraphrasiert dabei die vorangegangene Frage von Unid1 noch einmal, indem er den Zusammenhang von Chip und Nummer für die Platzzuweisung zusammenfassend darstellt, ,AUF DEN PLATZ SETZEN (.) MIT DER NUMMER AUF DEM CHIP“. Als Reaktion auf eine zweite im Video akustisch jedoch unverständliche Frage von Unid2 (Zeile 7) fügt Tim seinen Ausführungen zur Platzzuweisung einen weiteren Aspekt hinzu. Mit der Aussage, ,,stehen an der wand" (Zeile 8), spezifiziert er den Ort, an dem die mit dem Chip korrespondierenden Nummern im Labor zu finden sind.

In der oben dargestellten Sequenz (Zeile 5-8) sind dabei deutliche Lautstärkewechsel erkennbar. Die erste Antwort von Tim, , auf den Platz setzen mit der nummer auf dem chip“ (Zeile 6), wird als Replik auf eine leise gestellte Frage (Zeile 5) sehr laut und deutlich gesprochen. Die zweite Antwort, ,stehen an der wand“ (Zeile 8), hingegen ist deutlich leiser und schneller. Der erste Teil der Frage ist somit für alle Wartenden leicht hörbar und stellt zugleich eine an alle TeilnehmerInnen adressierte Handlungsanweisung dar. Die Nummer auf dem Chip gibt somit an, welchen Arbeitsplatz die Eintretenden im Laborraum (auf)suchen und einnehmen sollen. Die zweite, leiser gesprochene Antwort ist aufgrund ihrer differenten Lautstärke nicht für alle hörbar. Tim richtet seine Antwort hier an eine ganz bestimmte Person vor dem Labor, die hier als Unid2, bezeichnet wird. Mit seiner Antwort erklärt Tim Unid2, wo die eben thematisierten Nummern im Labor zu finden sind. Dieser deutliche Lautstärkewechsel in Tims Antwortverhalten bei der Informationsweitergabe an alle oder an einzelne ProbandInnen zeigt sich auch im Rahmen des Experiments als ein typisches situatives Merkmal, welches zur Vereinzelung der ProbandInnen und zur Kontrolle der weitergegebenen Informationen genutzt wird. Auf der Ebene der situativen Realisierung wird dieser Aspekt der gezielten Lautstärkevariation im Zusammenhang mit dem Aspekt des typischen Stellens und Beantwortens von Fragen (Abschnitt 5.4.3) noch einmal aufgegriffen.

Der oben angesprochene Chip, welcher mit einer Nummer versehen ist, stellt dabei in seinen unterschiedlichen Funktionen zugleich ein Merkmal der Binnenstruktur und der situativen Realisierungsebene dar. Einerseits ist er für die 
Standardisierung der Platzzuweisung zu Beginn des Experiments von entscheidender Bedeutung (siehe Abschnitt 5.3.2): Bevor die eingeladenen ProbandInnen das Labor betreten, haben sie bereits alle einen kleinen kreisförmigen Holzchip aus einem schwarzen Stoffbeutel gezogen. Dieser ist mit einer Nummer versehen. Wie im Redezug von Tim deutlich wird, korrespondiert die Nummer auf diesem Spielchip mit den Nummern an den Kabinen im Labor. Das materielle Objekt des Chips besitzt hier somit eine kommunikative Funktion. Der Spielchip zeigt den Teilnehmenden auf diese Weise an, welchen Arbeitsplatz sie im Labor einnehmen sollen. Durch diesen Mechanismus gelingt es, die Versuchsteilnehmer koordiniert auf die einzelnen Arbeitskabinen zu verteilen. Im Rahmen der Vorbereitungen des Experiments muss dabei die Anzahl und Auswahl der Zahlenchips im Stoffbeutel gewissenhaft mit der Auswahl der im Labor zu besetzenden Arbeitsplätze abgestimmt werden.

Es entscheiden somit weder ExperimentatorInnen noch die Teilnehmenden darüber, welchen Platz die einzelnen Personen im Labor einnehmen - stattdessen wird dies durch den zufällig gezogenen Spielchip determiniert. Dieser Prozess der Platzzuweisung setzt damit den außenstrukturellen wissenschaftlichen Anspruch von Randomisierung und Anonymisierung im Labor um, welcher an den wissenschaftlichen Paradigmen der Methodologie sozialwissenschaftlicher Experiment orientiert ist (Abschnitt 2.1). Mit dem Ziehen des Chips wird ihnen einerseits per Zufall ein Platz im Labor und in vielen Experimenten zugleich auch eine Rolle im Experiment zugeordnet. Zugleich erfolgt in diesem Moment die Entkopplung von personenbezogenen und experimentbezogenen Daten. Durch die zufällige Zuordnung, die nicht dokumentiert wird, ist es nachfolgend unmöglich, die persönlichen Daten der VersuchsteilnehmerInnen mit ihren im Experiment getätigten Entscheidungen zu verknüpfen. Die Entscheidungen der ProbandInnen werden somit als anonymisiert betrachtet.

Der Spielchip stellt in diesem Rahmen eine Objektivation für den Prozess der Randomisierung und Anonymisierung dar. Er kann hier als Objektivation betrachtet werden, da sich in seiner Verwendung das Wissen und der Anspruch der Forschenden ausdrückt, die Platzzuordnung der Teilnehmenden auf eine ganz spezifische Art zu koordinieren und zu kontrollieren.

Er dient hier somit auf binnenstruktureller Ebene als standardisiertes Mittel, um diesen außenstrukturellen wissenschaftlichen Anspruch der Randomisierung und Anonymisierung im Rahmen des Laborexperiments praktisch umzusetzen. Zugleich wirkt der jeweils spezifische Spielchip, den die ProbandInnen in den Händen halten, jedoch auch als Element der situativen Realisierung, da er es den ExperimentatorInnen und ProbandInnen ermöglicht, ihren ganz spezifischen Arbeitsplatz im Raum zu ermitteln (siehe Abschnitt 5.4.1). 


\section{Die Platzsuche der ProbandInnen}

Abb. 5.5a Platzsuche 2

(Totale)

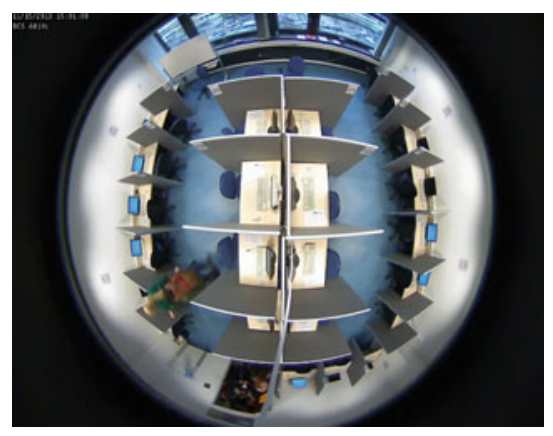

Abb. 5.5b Platzsuche 2 (Zoom)

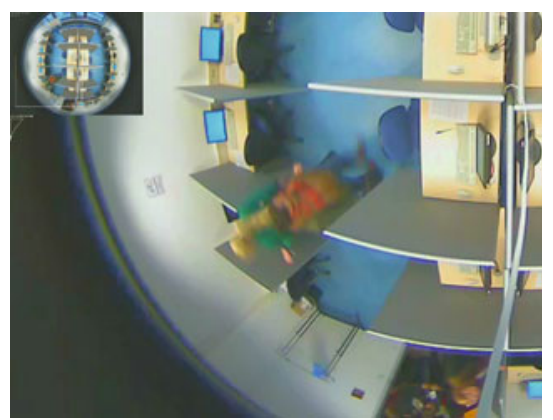

Die Abbildungen 5.5a und 5.5b zeigen wie die ersten Probandinnen das Labor betreten. Man sieht Silke, eine junge Frau, die Tim gerade das Papierblatt reichte. Sie schaut ihn noch kurz an und betritt dann das als erste Probandin das Labor. Zielstrebig wählt sie den linken Gang des Laborraums und kreuzt dabei den Weg von Juliane, welche sich mit einem Papierbecher in der Hand wieder an die untere Seite des Raums begibt. Beide Personen wenden ihre Körper im Augenblick ihrer Begegnung leicht voneinander ab. Die Experimentatorin Juliane geht wieder zurück zur Eingangstür des Labors. Danach verbleibt sie links neben der geöffneten Tür und beobachtet das Eintreten weiterer TeilnehmerInnen. Die Probandin Silke hingegen setzt sich an den Platz 4. Auf ihrem Stuhl sitzend, zieht sie ihre Jacke aus. Ihr Blick bleibt dabei auf das innere ihrer Kabine ausgerichtet.

Durch ihre Körperformation zeigen die beiden Handelnden hier an, dass sie keine zentrierte Interaktion anstreben. Trotz ihrer körperlichen Nähe ist eine gegenseitige Begrüßung in dieser Kommunikationssituation nicht üblich. Die beiden Frauen 
wenden sich stattdessen voneinander ab und verfolgen jeweils differente Handlungsziele. Die Handlungssequenz der Platzsuche der ProbandInnen umfasst spezifischer betrachtet 20 Handlungssequenzen. Auch wenn es sich um eine Situation körperlicher Kopräsenz mit den anderen eintretenden ProbandInnen handelt, bewältigen die ProbandInnen die Suche und das Finden ihres Arbeitsplatzes dabei individuell und auch durchaus unterschiedlich. Charakteristische Momente der hierbei zu beobachtenden Handlungen verweisen dabei auf allgemeine Merkmale und Muster einer zielgerichteten Orientierung in einem Raum. Auffällig ist jedoch, mit welcher Zielstrebigkeit sich die große Mehrheit der ProbandInnen im Raum bewegt und wie schnell sie ihren Arbeitsplatz finden. Bezogen auf die Gesamtzahl der Teilnehmenden passiert es nur sehr vereinzelt, dass die ProbandInnen bei ihrer Suche innehalten, sich umdrehen oder einen Weg im Labor zweimal beschreiten, bis sie an ihren Sitzplatz gelangen.

Diese mehrheitlich zielstrebige Orientierung der ProbandInnen legt die Interpretation nahe, dass die ProbandInnen bereits über Vorerfahrungen mit der Orientierung im Laborraum verfügen und aus diesem Grund bereits beim Betreten wissen, ob sie den linken oder rechten Gang des Labors nutzen müssen, um schnellstmöglich zu ihrem Arbeitsplatz zu gelangen. Die Vorerfahrung mit dem Laborraum und dem Ablauf der Experimente bilden eine zentrale Wissensgrundlage, die interaktiv nutzbar gemacht wird, und bildet zugleich einen Aspekt der Außenstruktur. Das Abschnitt 5.2.3.1 zur Außenstruktur bietet eine vertiefende Darstellung zu den teilnehmenden ProbandInnen und thematisiert ebenfalls die adressierten Aspekte von Vorwissen und Erfahrung mit der Durchführung von Experimenten.

\section{Die soziale Vereinzelung}

Abb. 5.6a Soziale Vereinzelung (1)

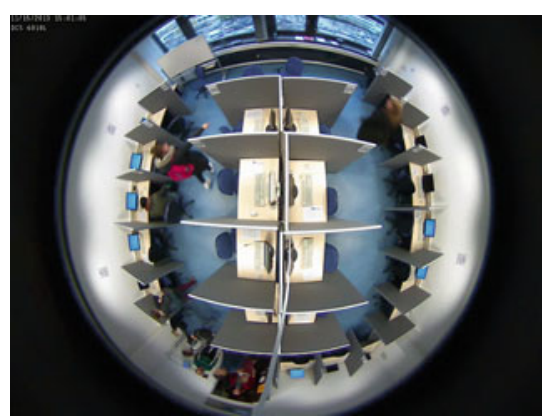


Abb. 5.6b Soziale

Vereinzelung (2)

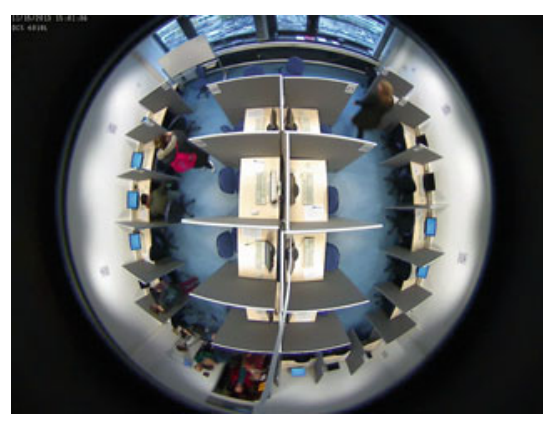

Abb.5.6c Soziale

Vereinzelung (3)

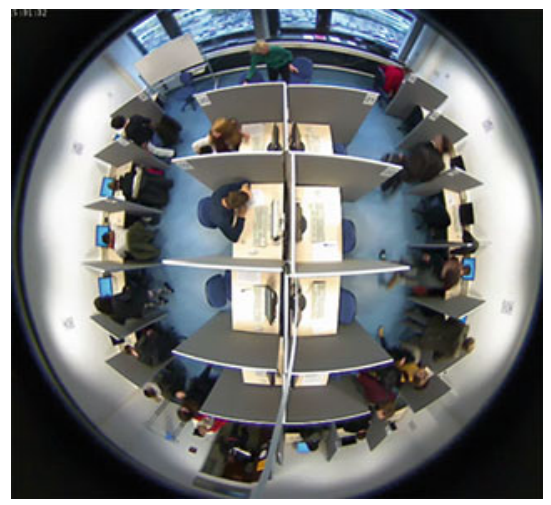

Der nachfolgende Abschnitt zeichnet nach, wie die soziale Vereinzelung der Probandinnen im Zuge der Platzsuche im Labor voranschreitet (Abb. 5.6a/5.6b/5.6c) Kurz nach der ersten Probandin Silke betreten zwei weitere Probandinnen, Ina und Jana, das Labor. Jana richtet sich schon beim Eintreten nach rechts aus. Sie läuft den ganzen rechten Gang entlang bis zur Fensterfront. Ina hingegen betritt den linken Gang. Sie wendet ihren Körper beim Laufen der mittleren Reihe der Arbeitsplätze zu. Nachdem Ina an den ersten beiden Kabinen vorbeigegangen ist, hebt sie ihren rechten Arm und weist mit ausgestrecktem Zeigefinger auf den Arbeitsplatz Nummer 20 (Abb. 5.6a). Sie schaut in Janas Richtung und sagt:

Z 9: Ina: du bist hier ((wendet sich zu Ina und macht eine Zeigegeste)) 
Im Anschluss daran knickt sie ihren rechten Arm ein, wendet ihren Kopf nach links und macht eine Zeigegeste in die linke Richtung zum Arbeitsplatz Nummer 5 (Abb. 5.6b). Sie kommentiert dies durch die Aussage:

Z 10: ach gott und ich bin hier

Darauf blickt sie wieder zu Jana und sagt lachend:

Z 11: das ist ja auch was ((lacht))

Ina wendet ihren Blick im Anschluss wieder von Jana ab und beginnt zugleich, ihren Rucksack abzusetzen. Sie stellt diesen an die Trennwand des Arbeitsplatzes. Jana blickt in Inas Richtung und läuft am Arbeitsplatz der ExperimentatorInnen vorbei und betritt von der Fensterseite den linken Gang. Sie wendet sich der oberen ersten Kabine mit dem Arbeitsplatz 21 zu, legt ihren Chip auf den Tisch und nimmt den dort befindlichen Stuhl ein. Sie wendet ihren Blick noch einmal kurz nach oben und scheint die Nummer dieses Arbeitsplatzes zu betrachten. Sie zieht ihre Jacke aus und dreht sich zu Ina um. Diese hat ihre Blickrichtung jedoch auf das Innere der Kabine ausgerichtet (Abb. 5.6c). Jana hängt ihre Jacke über die Lehne ihres Stuhls und setzt sich auf diesen. Sie wendet ihren Körper zweimal leicht nach rechts und dreht sich dann noch einmal zu Ina um. Diese zeigt keine Reaktion. Jana nimmt darauf die auf ihrem Platz ausliegenden Instruktionen zur Hand und beginnt, darin zu lesen.

Auch wenn im vorangegangen Abschnitt zur "Platzsuche der ProbandInnen“ ausgeführt wurde, dass das Betreten des Labors durch die einzelnen ProbandInnen in je einzelne Handlungssequenzen unterteilt werden kann, sind die Handlungen der Probandinnen Ina und Jana hier miteinander interaktiv verwoben. Sie sind die einzigen beiden Probandinnen, die beim Betreten des Labors miteinander verbal in Kontakt treten. Diese kurze Interaktion zwischen den beiden Probandinnen verweist darauf, dass die Teilnehmenden die Platzzuordnung durch den nummerierten Spielchip und die damit gegebene Handlungsanweisung verstehen und anerkennen. Auch wenn sich Teilnehmende kennen sollten, haben sie nicht die Möglichkeit, Arbeitsplätze zu wählen, die nebeneinanderliegen. Zugleich wird durch Inas Aussage, ,du bist hier“ (Zeile 9) und ihre damit verbundenen körperlichen Entäußerungen ersichtlich, dass sie bereits vor Betreten des Labors Kenntnis davon hatte, welche Nummer der Spielchip von Jana hat und sie so darauf aufmerksam machen kann, wo sich ihr Arbeitsplatz befindet. Dass Ina die räumlich nahe Positionierung der beiden Arbeitsplätze mit ihrer Aussage, ,ach gott und ich bin hier“ (Zeile 10) sowie daran anschließend mit, „das ist ja auch was“, 
als amüsanten Zufall darstellt, verweist darauf, dass es für sie überraschend ist, dass sie sich beide gegenübersitzen. Die Probandin weiß somit darum, dass dies weder wahrscheinlich noch beeinflussbar ist. Es entäußert sich hier somit der im Zuge der Außenstruktur thematisierte Aspekt der Randomisierung und damit der zufälligen Verteilung der ProbandInnen im Labor sowie die Kenntnis, die auch die teilnehmenden ProbandInnen von diesem Merkmal der Laborsituation haben. Dieses Beispiel wird in der Analyse unter Abschnitt 5.4.1 noch einmal aufgegriffen.

Nachdem beide Probandinnen ihren Arbeitsplatz erreicht haben, schaut Jana sich mehrfach nach Ina um. Das Ende der Interaktionssituation wird daran ersichtlich, dass auch Jana zu einer anderen Handlung übergeht. Nachdem sie mehrfach versucht, einen Blickkontakt mit Ina herzustellen, nimmt sie sich die Instruktionen und richtet ihre Aufmerksamkeit auf diese. Die Kommunikation bricht ab, da Jana keine Reaktion auf Ina zeigt. Diese kurze Handlungssequenz stellt exemplarisch dar, wie sich der Aufmerksamkeitsfokus der beiden Probandinnen verschiebt und sich von einer Situation des Miteinanders zu einer Situation des Nebeneinanders wandelt. Dass das Betreten des Labors und die Einnahme der Arbeitsplätze mit der Vereinzelung der ProbandInnen einhergehen, kann als ein typisches binnenstrukturelles Gliederungsmerkmal im Ablauf der Experimente im Labor betrachtet werden.

\section{Die überschüssige ProbandInnen}

Durch das Eintreten der letzten ProbandInnen in das Labor wird der Blick auf den Türbereich freigegeben. Tim steht auf der linken Seite des Türbereichs und wendet dem Labor den Rücken zu. Nachdem der letzte Teilnehmende das Labor betreten hat, dreht Tim seinen Körper nach rechts und wendet sich einer zweiten Person zu, die am rechten Rand des Türbereichs steht (Abb. 5.7a/5.7b). Während Tim den Stapel von Papieren sortiert, den er in der Hand hält, sieht und hört man, dass diese beiden Personen in eine Interaktion verwickelt sind.

Z 12: Unid3: (... 0.5$)$

Z 13: Tim: die anwesenheitsliste ist jetzt voll. 
Abb. 5.7a überschüssige ProbandInnen 1 (Totale)

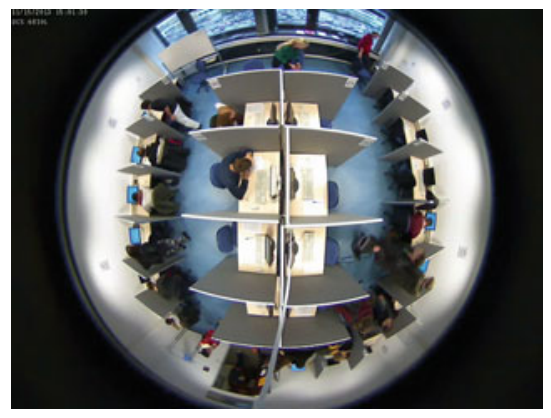

Abb. 5.7b überschüssige ProbandInnen 1 (Zoom)

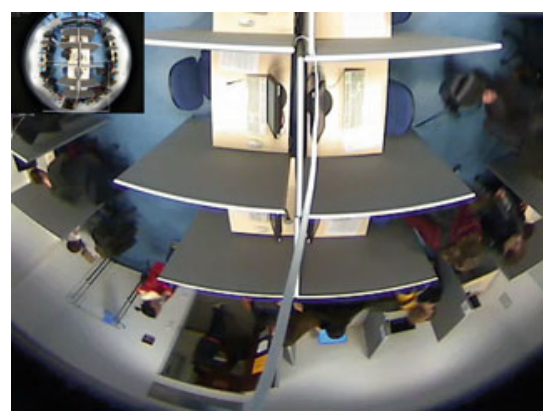

Abb. 5.7c überschüssige ProbandInnen 2 (Totale)

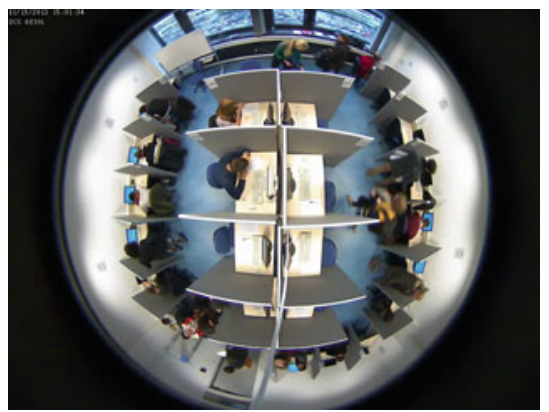


Abb.5.7d überschüssige ProbandInnen 2 (Zoom)

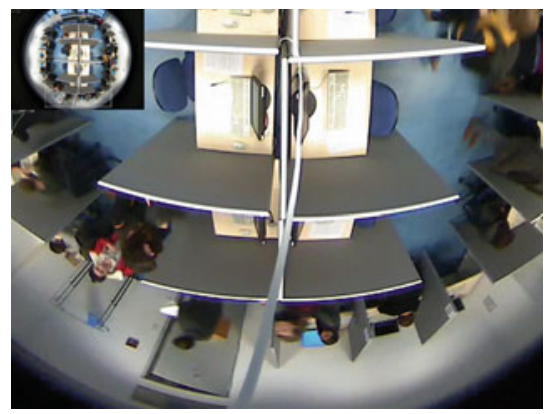

Während Tim diese Worte spricht, schiebt er den Stapel seiner Papiere zusammen und tritt näher an die zweite Person heran. Er blickt zu Unid3, hebt seinen Arm, streckt ihr die Hand entgegen und sagt:

Z 14: Tim: bitte füll das da noch aus.

Danach senkt er seinen Arm wieder, dreht sich von Unid3 weg und wendet sich der geöffneten Labortür zu. Während Tim seinen Körper schon auf eine neue Laufrichtung ausgerichtet, ist sein Kopf noch zu Unid3 gewendet. Beim Durchschreiten des Türbereichs sagt er:

Z 15: Tim: danach komm ich gleich raus

Tim betritt darauf den Laborraum, greift mit der linken Hand nach der Tür des Labors und schließt diese hinter sich (Abb. 5.7c/5.7d).

Diese kurze Handlungssequenz (Zeile 12-15) verweist auf den standardisierten Umgang mit diesem Teil des Vorbereitungsablaufs. Weder Tim noch die vor dem Labor wartende Person zeigen sich sonderlich überrascht davon, dass sich vor dem Labor mehr Personen eingefunden haben, als für das Experiment benötigt werden. Dies zeigt sich unter anderem daran, dass Tim keine längeren Erklärungen gegenüber Unid3 darüber abgibt, warum 20 Personen das Labor betreten durften, Unid3 jedoch vor der Tür bleiben muss. Tim begnügt sich hier stattdessen mit einer kurzen Erklärung und nimmt an, dass diese ausreicht, um Unid3 zu den von ihm gewünschten Handlungen zu bewegen. Es wird ersichtlich, dass der Experimentator Tim sich bereits vor Beginn des Einlasses der ProbandInnen auf dieses Ereignis vorbereitet hat. Denn die benötigten Unterlagen, die Unid3 ausfüllen muss, hält er vor Ort bereithält, ohne seine Position an der Tür seit dem 
Beginn des Auftaktes der Situation verlassen zu haben. Diese Handlungssequenz verweist dabei auf den außenstrukturellen Aspekt der beiderseitigen Erwartungen und Kenntnisse von Labormitgliedern und ProbandInnen, sowohl hinsichtlich des typischen Ablaufs der Experimente als auch mit Bezug auf den Einlassprozess (siehe Abschnitt 5.2.4).

Durch die Explizierung einiger Kontextaspekte der Vorbereitung von Laborexperimenten und der Einladung von ProbandInnen kann diese Handlungssequenz zudem als Resultat und zugleich als Beispiel weiterer zentraler Aspekt der kommunikativen Konstruktion der Laborsituation gedeutet werden. Hier wie auch in vielfältigen anderen Sequenzen zeigt sich, wie die Forschenden darauf abzielen, das auf unterschiedliche Weise mediatisierte kommunikative Handeln gegenüber den Teilnehmenden zu standardisieren. So ist diese Handlungssequenz und der Umgang mit der wartenden Person Ausdruck des typischen und für beide Seiten verlässlichen Wechselspiels von positiven und negativen Sanktionen bzw. Sanktionsandrohungen, die charakteristisch für die Beziehung zwischen Forschenden und ProbandInnen ist.

In der Vorbereitung der Experimente werden stets mehr ProbandInnen eingeladen, als für die Durchführung der Experimente benötigt werden. Auf diese Weise versuchen die Forschenden sicherzustellen, dass die benötigte Anzahl von ProbandInnen auch wie geplant für die Datenerhebung im Labor zur Verfügung steht. Je nach Forschungsinteresse werden dabei gezielt bestimmte Gruppen des registrierten ProbandInnenpools ausgewählt und eingeladen. Die Einladung erhalten die ausgewählten Personen per E-Mail. Die Bestätigung der angemeldeten ProbandInnen wird als verbindliche Zusage betrachtet. Ein zu spätes Absagen oder Nichterscheinen wird mit einem negativen Eintrag in das persönliche Konto der ProbandInnen sanktioniert. Diese Minuspunkte verringern die Chance, zu weiteren Experimenten eingeladen zu werden. Die Eingeladenen werden darüber informiert, dass stets mehr TeilnehmerInnen eine Zusage für das Experiment erhalten, als tatsächlich benötigt werden. Am Experiment selbst nehmen diejenigen teil, die im Labor zuerst vor Ort sind und sich bei dem Mitarbeiter als Eingeladene ausweisen können. Wer rechtzeitig am Labor eintrifft, es jedoch nicht schafft, sich anzumelden, bevor alle freien Plätze im Labor vergeben sind, erhält eine geringe Aufwandsentschädigung von 5 Euro.

In einem typischen Wechselspiel wird die Attraktivität bestimmter Handlungsoptionen zielgerichtet kommunikativ beeinflusst. Im dargestellten Fall beispielsweise einerseits durch Belohnungen regelkonformen Handelns durch Geldgewinne in der Experimentteilnahme oder die Show-up fee, andererseits durch den Ausschluss vom Experiment und die Sanktionsandrohung des Eintragens eines Malus in die ProbandInnendatei. Der Prozess des Einladens und Anmeldens der 
ProbandInnen ist dabei ein maßgeblicher Aspekt der Einführung und des Trainings eines Rollenbildes, das zentral auf die Regelbefolgung der ProbandInnen abzielt. Die für das beobachtete Phänomen relevanten sozialen Rollen sind die der ExperimentatorInnen und die der ProbandInnen. Soziale Rollen stellen klassische außenstrukturelle Merkmale dar (siehe Abschnitt 5.2.3.1). In diesem Zusammenhang wird zugleich dargestellt, dass die kommunikative Konstruktion der Rollen der ProbandInnen und der ForscherInnen sich nicht erst im Labor selbst vollziehen, sondern diese bereits mit der Rekrutierung, der Registrierung und Einladung der ProbandInnen eingeführt werden.

\section{(C) Begrüßung und Belehrung}

\section{Die Begrüßung}

Abb. 5.8a Begrüßung 1

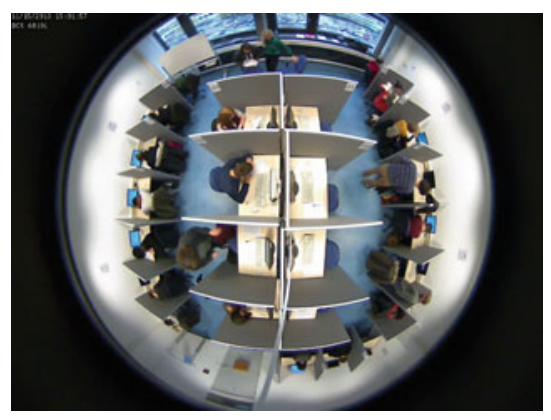

Abb. 5.8b Begrüßung 2

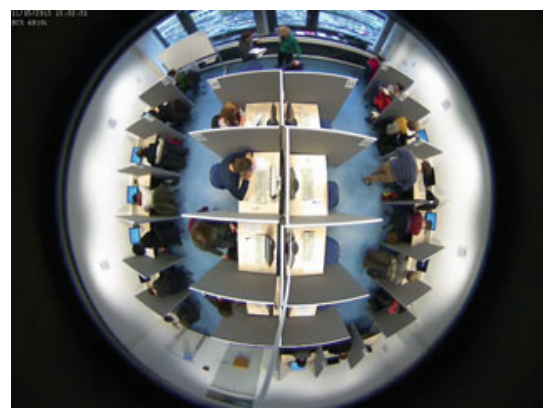


Abb. 5.8c Begrüßung 3

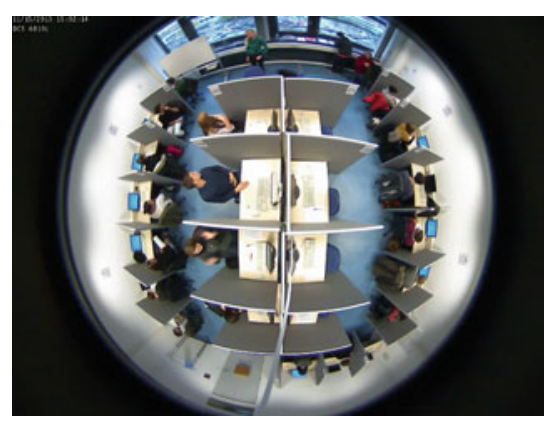

Die Tür des Laborraums ist geschlossen und alle Teilnehmenden haben ihre Sitzplätze erreicht. Die letzten ProbandInnen nehmen ihre Sitzplätze ein. Einige ProbandInnen sind noch damit befasst ihre Jacken auszuziehen. Tim läuft mit schnellen Schritten den rechten Gang in Richtung der Fensterseite nach oben. In der Hand hält er einen Stapel mit Papieren. Juliane sitzt am Arbeitsplatz der ExperimentatorInnen auf dem rechten der beiden Drehstühle. Tim läuft hinter ihr vorbei und positioniert sich hinter dem linken Drehstuhl. Juliane dreht ihren Stuhl zu Tim, wendet sich ihm zu und steht auf (Abb. 5.8a). Stehend betrachten beide den linken Computerbildschirm an ihrem Arbeitsplatz und wenden sich dann einander zu. Tim sagt etwas in der Videoaufnahme unverständliches:

Z 16: Tim: (...) ((macht mit der linken Hand eine Zeigegeste in Julianes Richtung))

Nun zeigt Tim mit seinem linken Zeigefinger auf Juliane und klappt die Handfläche darauf ein, sodass alle seine Finger in Richtung seines Körpers zeigen. Juliane nickt als Antwort auf seine Geste (Abb. 5.8b). Tim wendet sich von ihr ab, legt die in seiner Hand befindlichen Papiere auf der Fensterbank ab und beginnt dort unterschiedliche Papiere zu sortieren. Juliane wendet ihren Körper und damit auch ihre Blickrichtung erst auf die linke und danach auf die rechte Seite des Raumes. Nach rechts ausgerichtet beginnt sie zu sprechen:

\section{Z 17: Juliane: SO, herzlich willkommen zu dem experiment}

Während die Experimentatorin Juliane zu sprechen beginnt, richtet sie ihren Körper sichtlich auf und tritt einen Schritt hinter den Stuhl zurück. Die Körperpositionen der ProbandInnen im Labor sind zu diesem Zeitpunkt auf das Innere 
ihrer Kabinen ausgerichtet. Als die Experimentatorin zu sprechen beginnt, wenden einzelne ProbandInnen den Kopf in ihre Richtung. Die Mehrheit blickt jedoch weiterhin in ihre Kabinen. Die Experimentatorin schaut noch einmal auf die rechte Seite des Laborraums, dreht sich dann aber deutlich auf die linke Seite und fährt fort:

Z 18: Juliane: wenn ihr (räuspern) euch jetzt alle eingefunden habt, dann kann es ja jetzt losgehen.

Wie auf der Abbildung 5.8c zu sehen ist, richten die ProbandInnen auf der linken Seite nun vermehrt ihre Aufmerksamkeit auf die Experimentatorin und schauen sie an. Einige der ProbandInnen rollen mit ihren Drehstühlen ein Stück rückwärts aus der Kabine heraus, um die Experimentatorin besser zu sehen. Die auf der rechten Seite befindlichen ProbandInnen ändern ihre Körperpositionierung hingegen kaum. Nur bei den ProbandInnen an den Arbeitsplätzen 8, 10 und 11 lässt sich beobachten, dass sie kurz die Experimentatorin anschauen und ihr den Kopf zuwenden. Im Anschluss gehen jedoch auch diese ProbandInnen wieder in ihre Ausgangsposition über und wenden sich dem Inneren der Kabinen zu.

Im Übergang von Zeile $17 \mathrm{zu}$ Zeile 18 verlässt die Experimentatorin ihre mittige Positionierung und tritt einen Schritt nach links. Nun befindet sie sich hinter dem linken der beiden Stühle. Sie wendet sich nun deutlich der linken Seite des Laborraums und den dort sitzenden ProbandInnen zu.

Z 19: Juliane: ihr habt ja auf eurem platz alle ein paar instruktionen zu liegen $\mathrm{Z}$ 20: Juliane: und wir werden auch gleich damit starten.

Einige der ProbandInnen auf der linken Seite des Laborraums wenden daraufhin ihren Blick von der Experimentatorin ab und nehmen die vor ihnen liegenden Blätter in die Hand.

Die hier dargestellte Handlungssequenz (Zeile 16-20) verweist dabei auf drei interessante Aspekte. Einerseits stellt die Begrüßung der teilnehmenden ProbandInnen ein binnenstrukturelles Gliederungsmerkmal (Abschnitt 5.3.1) im Verlauf des Laborexperiments dar. Die Zuständigkeit für diesen Gliederungspunkt stimmen die beiden ExperimentatorInnen Tim und Juliane mittels mimischer und gestischer Mittel ab. Dies ist notwendig, da es für diesen Gliederungspunkt typische Redezugbestimmungen gibt, die vorsehen, dass genau ein Mitglied des ExperimentatorInnenteams diese Begrüßung der Teilnehmenden übernimmt. Tim macht zur Abstimmung der beiden mit der linken Hand eine Zeigegeste in Julianes Richtung und diese nickt zustimmend. Tim wendet sich im Folgenden von Juliane 
$a b$ und widmet sich dem Sortieren von Papieren auf der Fensterbank. Juliane hingegen schaut noch einmal kurz auf die linke und dann auf die rechte Seite des Raums und beginnt dann ihren Monolog mit einem lauten und deutlichen „SO“ (Zeile 17). Durch ihre folgenden Aussagen (Zeile 17-20) markiert die Experimentatorin den Übergang zwischen unterschiedlichen Handlungssequenzen. Die offizielle Begrüßung, ,,herzlich willkommen zu dem experiment“ (Zeile 17), stellt dabei den Auftakt der offiziellen Interaktion zwischen der Experimentatorin und den ProbandInnen dar. Das Gliederungsmerkmal der Platzsuche und die damit verwobenen Handlungen gelten als abgeschlossen, wie die Experimentatorin in Zeile 18 mit, ,wenn ihr (räuspern) euch jetzt alle eingefunden habt, dann kann es ja jetzt losgehen“, deutlich markiert. Durch ihre Aussagen (Zeile 17-20) ordnet die Experimentatorin die vorausgegangenen Aspekte als Teile der Präsequenz des nachfolgenden Experiments ein.

Zweitens wird mit der Aussage, ,ihr habt ja auf eurem platz alle ein paar instruktionen zu liegen“"(Zeile 19), auf ein zentrales Objekt im Rahmen der Laborexperimente verwiesen. Bei den Instruktionen handelt es sich, wie oben in den Ausführungen zur Ausstattung des Labors (siehe Beginn dieses Abschnitts II) kurz dargestellt, um mehrere bedruckte A4 Seiten. Sie stellen im Rahmen der Experimente ein textliches Medium dar, mittels welchem den Teilnehmenden aus Sicht der Forschenden alle relevanten Informationen über das nachfolgende Experiment und die Auszahlungsmodalitäten vermittelt werden. Für die Analyse des Laborexperiments aus gattungsanalytischer Perspektive stellen die Instruktionen ein zentrales binnenstrukturelles Merkmal dar. Verschiedene außenstrukturelle Aspekte wie die Standardisierung des Experimentablaufs, das Framing- und Akteursmodell der ökonomischen ForscherInnen oder der Anspruch der dichten Replikation bestehender Experimente entäußern sich in den Instruktionen und werden hier analytisch greifund beschreibbar. Zudem treten die Instruktionen auch auf der Ebene der situativen Realisierung wiederkehrend als relevante Objektivation auf, wenn bei Nachfragen auf diese als Informationsquelle verwiesen wird. Durch die anschließende Aussage der Experimentatorin, ,und wir werden auch gleich damit starten“ (Zeile 19), wird die Beschäftigung mit den Instruktionen als ein weiterer binnenstruktureller Gliederungsaspekt im Ablauf des Experiments adressiert.

Drittens zeigt diese Sequenz (Zeile 16-20) auf, welche Herausforderungen, Potenziale und Konsequenzen die spezielle räumliche Struktur des Labors für die ablaufenden Interaktionen bietet. Sowohl die ProbandInnen als auch die ExperimentatorInnen sind in ihrer Sicht durch die Trennwände und Kabinen beschränkt. Während die Experimentatorin Juliane zu Beginn der Handlungssequenz am Arbeitsplatz der ExperimentatorInnen sitzt, steht sie mit Beginn der Interaktion mit Tim auf und behält im Folgenden eine stehende Körperposition bei. Das mehrmalige Wenden 
ihrer Blickrichtung vor dem Beginn ihres Redezug in Zeile 17 und auch im Übergang zwischen Zeile 17 und 18 ist durch die visuellen Barrieren begründet, welche die Trennwände im Labor darstellen. Nur so gelingt es ihr zu überprüfen, ob alle ProbandInnen ihre Arbeitsplätze im Labor eingenommen haben und zum nächsten Gliederungspunkt übergegangen werden kann. Auch die Teilnehmenden reagieren deutlich auf die Beschränkungen ihrer visuellen Wahrnehmung. Um die Experimentatorin sehen zu können, müssen die ProbandInnen sich ihr aktiv zuwenden und die im Experiment vorgesehene Körperausrichtung auf das Innere der Kabinen unterbrechen. Während die auf der rechten Seite befindlichen ProbandInnen dies mehrheitlich unterlassen, wenden sich auf der linken Seite bereits kurz nach Beginn des Redezuges (ab Zeile 18) deutlich mehr Personen der Experimentatorin zu. Darauf reagierend, verlässt die Experimentatorin ihre mittige Positionierung und tritt einen Schritt nach links und richtet ihre Körperposition im weiteren Ablauf der Handlungssequenz (Zeile 18-20) deutlich auf die linke Seite aus. Dass sich die Experimentatorin hier für eine Seite des Laborraums entscheidet und sich dieser aktiv zuwendet, ist ein typisches Merkmal der situativen Realisierungsebene. Es ist Ausdruck des Umgangs mit den visuellen Barrieren, die mit den räumlichen Gegebenheiten im Labor zusammenhängen (Abschnitt 5.3.7). Die Ausrichtung der Experimentatorin auf die linke Seite des Laborraums und die vermehrte Zuwendung von TeilnehmerInnen auf dieser Seite des Raums stellt somit ein interaktives Wechselspiel zwischen der Experimentatorin und den ProbandInnen dar.

\section{Die standardisierte einleitende Belehrung}

Abb. 5.9a Einleitende Belehrung (1)

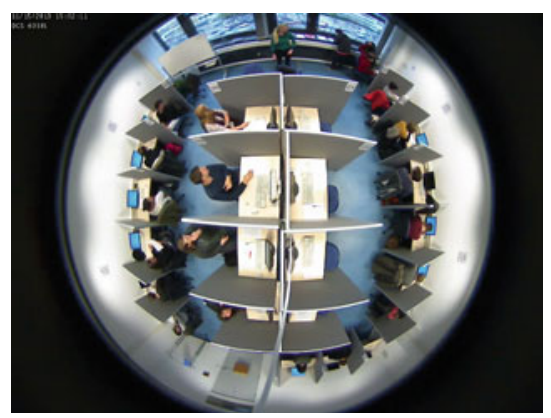


Abb. 5.9b Einleitende

Belehrung (2)

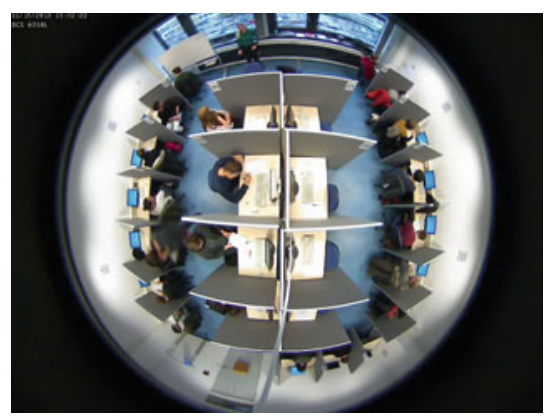

Die Experimentatorin Juliane schaut leicht nach rechts in Tims Richtung (Abb. 5.9a). Dieser ist noch immer damit beschäftigt, die auf der breiten Fensterbank ausliegenden Papiere zu sortieren. Er nimmt einige Papiere in die Hand und wendet sich Juliane zu. Tim läuft mit einigen Blättern in der Hand vor Juliane vorbei in Richtung Labortür (Abb. 5.9b), öffnet diese und verlässt den Laborraum. Die Experimentatorin tritt einen Schritt nach hinten und setzt ihren Monolog fort:

Z 21: kurz noch zu den ALLgemeinen regeln (.)

$\mathrm{Z}$ 22: grundsätzlich ist es so, dass ihr bitte nicht miteinander kommunizieren sollt.

Z 23: jeder macht das sozusagen für sich allein (.)

$\mathrm{Z}$ 24: ähm des weiteren sollen bitte keine elektronischen geräte benutzt werden

$\mathrm{Z} 25$ : und sollte es fragen geben unklarheiten $>$ wie auch immer<

$Z$ 26: dann gebt mir bitte ein zeichen oder uns

Z 27: >damit wir dann zwischendurch nicht so einen zeitdruck haben<

$Z$ 28: und dann kommen wir einzeln zu euch und beantworten die fragen.

Z 29: sollte die frage so wichtig sein, dass sie für alle geklärt werden muss, dann stellen wir

Z 30: sie noch einmal laut oder beantworten sie noch mal laut

Z 31: und dann lassen wir alle anderen das auch wissen.

Z 32: ansonsten könnt ihr jetzt bitte starten die instruktionen zu lesen und wenn wir dann

Z 33: fertig sind, dann beginnt das experiment.

Z 34: danke

Nachdem die Experimentatorin ihren Redezug beendet hat, tritt sie wieder an den Arbeitsplatz der ExperimentatorInnen heran, dreht mit ihrer linken Hand die 
Lehne des rechten Drehstuhl zu sich um und nimmt dort Platz. Auch die ProbandInnen, die vorher zur Experimentatorin schauten, wenden sich nun von ihr ab und dem Inneren ihrer Kabinen zu und senken ihre Köpfe.

Die hier dargestellte Handlungssequenz ist Teil der standardisierten BegrüBung, die auf sehr ähnliche Weise in allen Experimenten vorgetragen wird. Es handelt sich hier um ein binnenstrukturelles Gliederungsmerkmal (Abschnitt 5.3.1), welches im Ablauf der Laborexperimente stets zu finden ist. Zentral ist hier die Nennung und Erklärung der ,allgemeinen Regeln“ (Zeile 20). Diese explizieren die drei grundlegenden offiziellen Verhaltensregeln im Labor: Erstens sollen die Teilnehmenden ,nicht miteinander kommunizieren“ (Zeile 21), zweitens dürfen „keine elektronischen Geräte“ (Zeile 23) benutzt werden und drittens wird darum gebeten, bei Fragen und Unklarheiten ein Zeichen zu geben, damit diese individuell beantwortet werden können (Zeile 24 und 25). In der hier betrachteten Handlungssequenz (Zeile 20-33) fehlt jedoch ein Aspekt, der sich in den ethnographischen Beobachtungen anderer Experimente bei diesem Gliederungsmerkmal typischerweise zeigte. So folgt der Nennung der drei Laborregeln (Zeile 20-30) meistens der Hinweis auf die drohende Sanktion bei Zuwiderhandlung der ProbandInnen. Diese besteht in der Gefahr des Ausschlusses vom Experiment und des Verlustes der erspielten Gewinne. ${ }^{4}$

Diese drei Regeln sind zugleich binnenstrukturelle Merkmale im Sinne einer Standardisierung der einleitenden Belehrung, mit denen sich Abschnitt 5.3.3 vertiefend befassen wird. Ihr allgemeines Ziel ist eine Kontrolle der Informationsbasis der ProbandInnen durch die ExperimentatorInnen. Allen ProbandInnen sollen die gleichen Informationen zur Verfügung stehen. Diese drei Regeln sind somit zugleich Entäußerungen der im Zuge der Außenstruktur diskutierten epistemischen Ansprüche. Zentral sind dabei die auf die Replizierbarkeit der Experimente abzielende Standardisierung und Kontrolle gegenüber unerwünschter Störfaktoren, welchen in der Methodik des Experiments ein entscheidender Stellenwert beigemessen wird.

Dies zeigt sich beispielsweise deutlich an der dritten Regel des Umgangs mit Fragen oder Unklarheiten. Das Nadelöhr der Informationsübermittlung sind die ExperimentatorInnen: Sie entscheiden darüber, wann eine Information an alle ProbandInnen weitergeleitet werden soll, indem sie die Frage beziehungsweise die erfragte notwendige Information allen ProbandInnen durch eine laute und deutliche Ansage mitteilen. Auch hier zeigt sich deutlich der außenstrukturelle Aspekt der vorgängigen Setzung der im Labor vertretenen sozialen Rollen. Sowohl die ExperimentatorInnen als auch die ProbandInnen werden als zusammenhängende

\footnotetext{
${ }^{4}$ Dass dies keine leere Drohung ist, zeigt das Beispiel des Krisenfalls im Abschnitt 5.4.7.3.
} 
Gruppen angesprochen. Sprachlich ist diese Differenzierung durch die Verwendung der Personalpronomen ,ihr“ und „wir“ ausgedrückt. Mit den vertretenen sozialen Rollen hängen zugleich klar definierte Rederechte zusammen. Diese bestimmen darüber, wer wann und unter welchen Voraussetzungen im Rahmen der Experimente das Wort ergreifen darf. Wie die zugewiesenen Rederechte, die sich typischerweise im Experiment zeigen, interaktiv hervorgebracht und zur situativen Handlungskoordination eingesetzt werden, wird im Abschnitt 5.4.2 genauer betrachtet.

Wie die weitere Analyse zeigen wird, sind die drei zentralen Regeln jedoch mit weiteren Merkmalen der situativen Realisierungsebene verbunden, die für das Phänomen des Laborexperiments spezifisch sind. Im Zuge der Analyse wird dies am Bespiel des Stellens der individuellen Fragen (5.4.3) verdeutlicht. Verschiedenartige Techniken werden hier von den Beteiligten eingesetzt, um eine Regelbefolgung im gewünschten Sinne zu realisieren. So müssen die ProbandInnen die visuelle Barriere der Trennwände überwinden und sich für die ExperimentatorInnen sichtbar durch Handzeichen melden. Zugleich müssen auch die ExperimentatorInnen in regelmäßigen Abständen überprüfen, ob sie keine Meldung von ProbandInnen übersehen haben und ihre Position im Raum darauf ausrichten. Im Zuge der individuellen Beantwortung der gestellten Fragen zeigen sich überdies typische Techniken, die die ExperimentatorInnen im Zuge dieser Interaktionen verwenden, um beispielsweise die Sprechlautstärke der ProbandInnen zu regulieren.

Durch das Hinzuziehen ethnographischen Kontextwissens lässt sich zudem auch das Verlassen des Laborraums durch den Experimentator Tim zu Beginn der in diesem Abschnitt dargestellten Handlungssequenzen erklären. Tim verlässt hier den Raum, um zu dem vor der Tür wartenden Probanden Unid3 zurückzukehren. Er schließt mit seinem Verlassen des Raums an die Interaktion mit Unid3 und seine Aussage "danach komm ich gleich raus" (Zeile 15) an (vgl. obigen Abschnitt „Die überschüssigen ProbandInnen“).

Es überlagern sich hier zwei Handlungssequenzen und zugleich zwei Gliederungsmerkmale im Ablauf der Experimente. Die Experimentatorin Juliane widmet sich im Laborraum der Begrüßung und der standardisierten Belehrung der Teilnehmenden. Der Experimentator Tim hingegen ist mit der Registrierung der Teilnehmenden befasst. Das in den letzten beiden Handlungssequenzen mehrfach adressierte Sortieren von Unterlagen auf der Fensterbank durch den Experimentator Tim ist Teil dieses Handlungskomplexes und dient dem geordneten Ablegen der Anmeldungsunterlagen und dem Abgleich von angemeldeten und erschienenen ProbandInnen. 
Auch der Handlungskomplex der Registrierung der Teilnehmenden ist durch eine Abfolge typischer Gliederungsmerkmale charakterisiert. Tims Handlungen bilden hier den Abschluss des Gliederungsmerkmals „Umgang mit überschüssigen ProbandInnen und ihre Entlohnung“. Teil dieses Handlungsblocks ist es auch, die ProbandInnen zu entschädigen, die nicht am Experiment teilnehmen können, da alle benötigten Arbeitsplätze bereits besetzt sind. Auf diesen Aspekt verweist der Experimentator Tim auch mit seiner Aussage, ,die anwesenheitsliste ist jetzt voll“" (Zeile 13). Um zu bestimmen, ob Unid3 Anspruch auf die Entschädigung hat, ist es vorher jedoch nötig, zu kontrollieren, ob es sich bei den überschüssigen ProbandInnen auch um eingeladene ProbandInnen handelt. Denn nur die Einladung zu dem Experiment legitimiert den Anspruch auf eine Entschädigung bei Nichtteilnahme. Die Auszahlung des Entschädigungsbetrags in Höhe von 5 Euro erfolgt außerhalb des Labors. Um die Auszahlung zu erhalten, muss der Proband jedoch zuvor eine Quittung ausfüllen. Die Quittung hat Tim ihm bereits gereicht, bevor er das Labor betreten hat und ihn mit „,bitte füll das noch aus" (Zeile 14) dazu aufgefordert, die notwendigen Eintragungen zu machen (vgl. Abschnitt „Die überschüssigen ProbandInnen“). Auch dieses Beispiel illustriert anschaulich, welch entscheidende Bedeutung die Hinzuziehung ethnographischen Wissens für ein vertiefendes Verständnis der im Labor ablaufenden kommunikativen Konstruktionsprozesse hat. Einige zentrale Aspekte konnten durch das hier genutzte Videomaterial erfasst und durch die Deskription aufgezeigt wurden. Andere analytisch relevante Merkmale entziehen sich jedoch dieser Betrachtung, da sie außerhalb des Laborraums ablaufen oder - wie der Spielchip - im Videomaterial zwar sprachlich thematisiert, aber nicht gesehen werden können (vgl. Abschnitt „Der Spielchip als Koordinationsobjekt“").

Die hier betrachtete Eingangssequenz wurde herangezogen, um das Vorgehen zu illustrieren, welches in der Analysearbeit genutzt wurde. Um die nachfolgende Analyse trotz der Fülle der relevanten Beobachtungsaspekte nicht zu stark aufzublähen, wird diese dichte Darstellung des analytischen Vorgehens in den nachfolgenden Analysebeispielen nicht beibehalten. Stattdessen werden vermehrt kleine Sequenzen oder andere Formen der Thematisierung relevanter Aspekte gewählt. Teilweise wird dabei auch auf Beispiele zurückgegriffen, die aus diesem einleitenden Datenbeispiel stammen.

Wie im einleitenden Abschnitt dieses fünften Kapitels dargestellt, nutzt die hier vorliegende Arbeit eine Variante der Methode der Gattungsanalyse. Der Thematisierung des ethnographischen Kontexts wird dabei ein besonders großer Stellenwert für ein deutendes Verstehen der im Labor ablaufenden Handlungsprozesse eingeräumt. Im nachfolgenden Analyseteile wird der Einteilung der Gattungsanalyse in die Analyseebenen der Außenstruktur, Binnenstruktur und 
situativen Realisierung gefolgt, um so die systematische Beantwortung nach dem Prozess der Rahmenbildung im Labor nachzuvollziehen.

\subsection{Merkmale der Außenstruktur}

Die Außenstruktur einer kommunikativen Gattung bezeichnet den weiteren Kontext einer Handlungssituation. Bei der Außenstruktur handelt es sich um Kontextelemente, die das empirisch beobachtbare Geschehen im Labor zentral prägen, aus diesem selbst jedoch nicht ohne Weiteres abgeleitet werden können. Diese Wissensbestände sind institutionalisiert oder zumindest verfestigt und bilden die Charakteristika der sozialen Veranstaltung des wirtschaftswissenschaftlichen Laborexperiments. Eine Explizierung dieser Wissensbestände ermöglicht die Beantwortung von ,Warum-Fragen', die an das beobachtete empirische Phänomen der Rahmenbildung gestellt werden könnten. Warum ist Standardisierung, Randomisierung und Anonymisierung in Experimenten eigentlich von zentraler Bedeutung? Warum werden die Teilnehmenden eigentlich bezahlt und warum erhalten nicht alle den gleichen Geldbetrag? Warum dürfen die Teilnehmenden nicht miteinander kommunizieren?

Die Zielstellung des nachfolgenden Abschnitts besteht darin, zentrale Wissenselemente und -hintergründe des ,sozialen Milieus ' und der ,sozialen Veranstaltung “ der wirtschaftswissenschaftlichen Experimentalforschung im Labor darzustellen. Wie oben dargestellt, ist die Gattungsanalyse ein komparatives Verfahren. Ergebnisse und Erkenntnisse, die durch dieses Verfahren gewonnen werden, basieren auf einem Vergleich empirischer Situationen des fokussierten Kommunikationsphänomens. Die hier dargestellte Außenstruktur erfasst somit nicht alle Aspekte, die in einer spezifischen Handlungssituation als Kontext gelten und herangezogen werden. Sie weist vielmehr eben solche Momente aus, die sich in einem Vergleich als handlungsrelevant erweisen. Im Unterschied zu Anwendungen der Gattungsanalyse, die sich mit alltäglichen Kommunikationssituationen befassen, handelt es sich im Fall des wirtschaftswissenschaftlichen Laborexperiments jedoch um ein außeralltägliches Phänomen. Dies bedingt auch, dass das Alltagswissen nicht ausreicht, um das Phänomen in seiner Eigengesetzlichkeit zu entschlüsseln und analytisch aufzubrechen. Der empirische Untersuchungsgegenstand des wirtschaftswissenschaftlichen Laborexperiments erfordert aus diesem Grund eine Intensivierung und Erweiterung der Ebene der Außenstruktur.

Die im einleitenden Abschnitt 2.1 dargestellten Charakteristika des experimentellen sozialwissenschaftlichen Forschungsdesigns bilden auch im Fall des wirtschaftswissenschaftlichen Laborexperiments grundlegende außenstrukturelle Aspekte. Um 
in das Forschungsfeld des Experimentierens in den Sozialwissenschaften einzuführen, erfolgte die Thematisierung dieser Aspekte der Außenstruktur bereits zu Beginn der Arbeit. In einem Resümee des Abschnitt 2.1 können die gezielte Manipulation der Erhebungssituation, das Parallelgruppenmodell, welches zwischen Kontroll- und Versuchsgruppen unterscheidet und die Randomisierung der Versuchsteilnehmenden als zentrale Definitionskriterien bezeichnet werden. Aufbauend auf Isolation und Kontrolle der hypothesenrelevanten Variablen wird in einem Experiment allgemein angestrebt, spezifische Untersuchungsbedingungen zu schaffen, auf welche Forschende manipulierend eingreifen können, um den Einfluss interessierender Aspekte gezielt zu variieren. Im Sinne eines Ursache-Wirkungs-Prinzips wird davon ausgegangen, dass auf diese Weise eine kausale Analyse bestimmter Einflussfaktoren ermöglicht wird. Das Konstanthalten der Randbedingungen bildet dabei eine zentrale Grundlage, da nur so alternative Erklärungen für die Veränderungen des Untersuchungsgegenstandes ausgeschlossen werden können.

Es haben sich verschiedener Kontrolltechniken und -verfahren etabliert, durch deren Einsatz die Wirkung bekannter und auch unbekannter Störvariablen minimiert oder eine systematische Verzerrung der Ergebnisse ausgeschlossen werden soll. So zielt die Randomisierung bei der Zuweisung von einzelnen Personen zu Untersuchungsgruppen beispielsweise darauf ab, Störfaktoren auf der Ebene der Stichproben der Versuchspersonen auszuschalten. Andere Kontrolltechniken wie die Anonymisierung zielen hingegen auf die Eliminierung von Störfaktoren auf der Ebene der Durchführung der Experimente. Das Laborexperiment gilt dabei als die Variante des experimentellen Designs, welchem das höchste Maß an Kontrolle der Untersuchungssituation zugesprochen wird. Die Orientierung an den methodologischen Standards und Prinzipien sozialwissenschaftlicher Experimente erweist sich als prägend für den charakteristischen Prozess der Rahmenbildung des Laboratops. Wie die folgenden Kapitel zur Binnenstruktur (Abschnitt 5.3) und zur situativen Realisierung (Abschnitt 5.4) zeigen werden, wird der Befolgung von methodologischen und epistemischen Standards in der experimentellen Forschung ein sehr hoher Stellenwert beigemessen.

Die Grundzüge der Geschichte der experimentellen Wirtschaftsforschung sowie die Idee, dass sich wirtschaftliche Phänomene und Entscheidungsverhalten quantifizieren lassen, wurden bereits in der Feldeinführung (Abschnitt 2.2) dargestellt. Die dort beschriebenen Aspekte können gewissermaßen als ,Außenstruktur 
der Außenstruktur' betrachtet werden. Sie bilden damit den weiteren Kontext dessen, was sich im Prozess des wirtschaftswissenschaftlichen Laborexperiments als handlungswirksam erweist. ${ }^{5}$

Einen wichtigen außenstrukturellen Aspekt bildet das Akteursmodell der Forschenden, welches in der Konzeption der Experimente Anwendung findet. Abschnitt 5.2.1 skizziert aus diesem Grund den Wandel von der klassischen ökonomischen Perspektive des rationalen Homo oeconomicus hin zu der Aufnahme psychologischer Annahmen in Herbert Simons Konzept der ,bounded rationality“. Dieses Konzept bildet eine zentrale Grundlage für die experimentelle Forschung im Bereich der Wirtschaftswissenschaften und insbesondere der Verhaltensökonomie. Es prägt durch seine Anwendung dabei zugleich entscheidend die Modellierung von Entscheidungssituationen.

Aufbauend auf diese grundlegenden experimentellen und theoretischen Annahmen wird im weiteren Verlauf des Unterkapitels auf zwei zentrale Grundlagenkonzepte Bezug genommen: einerseits auf das im Anschluss an Kahneman und Tversky $(1981,1986)$ genutzte Konzept des Framings von Entscheidungssituationen (Abschnitt 5.2.2.1) und andererseits auf die „induzierte Werttheorie“ (Abschnitt 5.2.2.2) von Vernon Smith (1976). Auf diese geht, neben weiteren methodologischen Aspekten, auch die Nutzung des monetären Anreizes in wirtschaftswissenschaftlichen Experimenten zurück. Der Einsatz monetärer Incentives ist ein zentrales Definitionskriterium ökonomischer Experimente. Wie die Ausführungen zeigen werden, bilden diese beiden Konzepte grundlegende Paradigmen der wirtschaftswissenschaftlichen Laborforschung. Sie prägen die Konzeption von Experimenten im Labor und der dort stattfindenden Handlungsprozesse.

Aus gattungsanalytischer Perspektive bilden sich durch das soziale Milieu und das damit verbundene institutionelle Setting (Abschnitt 5.2.3) des untersuchten Kommunikationsphänomens wichtige außenstrukturelle Merkmale heraus. Diesem Aspekt entsprechend werden die typischen sozialen Rollen dargestellt (Abschnitt 5.2.3.1), die im ökonomischen Laborexperiment auftreten. Zugleich werden die Erwartungen und typischen Aufgaben thematisiert, die mit den phänomenspezifischen Personentypen verbunden sind. Im Rahmen von Laborexperimenten finden sich zwei klar voneinander differenzierte Rollen bzw. Personengruppen: die Forschenden oder ExperimentatorInnen einerseits und die

\footnotetext{
${ }^{5}$ Die hier vorgenommene Trennung zwischen Elementen, die der Außenstruktur zugehörig sind (5.2), und solchen, die zur ,Außenstruktur der Außenstruktur' (2.2) gezählt werden, verweist auf ein Spannungsverhältnis zwischen dem engen und dem weiten Kontext von Handlungssituationen bzw. -sequenzen. Das liegt jedoch an der grundlegenden Problematik, den handlungsrelevanten Kontext und seine Elemente abschließend zu bestimmen.
} 
ProbandInnen oder Forschungssubjekte andererseits. Beiden Typen von Handelnden werden dabei spezifische Charakteristika zugeschrieben. Im Zuge der Thematisierung der Rolle der ProbandInnen werden zudem einige Bemerkungen zum ProbandInnenpool des beobachteten Experimentallabors hinzugefügt.

$\mathrm{Zu}$ guter Letzt werden im Rahmen der Darstellung der Außenstruktur auch Aspekte des Laborexperiments expliziert, die zeitlich außerhalb der fokalen Beobachtungssituation des Geschehens im Labor liegen (Abschnitt 5.2.4). Im einleitenden Analysebeispiel wurde bereits an verschiedenen Stellen darauf verwiesen, dass die Prozesse der Rekrutierung, Registrierung und Entlohnung der ProbandInnen zwar nicht Teil der fokalen Analysesituation sind, diese jedoch gleichsam entscheidend prägen. So werden die ProbandInnen beispielsweise schon beim Anwerben durch Werbeplakate und Flyer ausdrücklich auf die monetäre Entlohnung hingewiesen und im Zuge der Rekrutierung und Registrierung wiederholt mit der Notwendigkeit der Regelbefolgung konfrontiert. Das typische Spannungsverhältnis von positiven und negativen Sanktion und die Rollenerwartungen, die an die ProbandInnen im Labor gestellt werden, treten bereits hier deutlich zutage und bilden so Wissensbestände, die zugleich das Geschehen im Labor prägen.

Die Bezugnahme und die Wirkung dieser außenstrukturellen Aspekte zeigt sich auf den Ebenen der Binnenstruktur und der situativen Realisierung, die sich empirisch erfassen lassen. Dieses Vorgehen ermöglicht es nachzuvollziehen, wie die außenstrukturellen Aspekte in der Situation transferiert werden und sich dort durch die kommunikative Konstruktion der geteilten Wirklichkeit der Handelnden entäußern. So fragen Binnenstruktur und situative Realisierung beispielsweise nicht danach, warum Standardisierung, Randomisierung und Anonymisierung zentrale Prinzipien der Forschung im Experimentallabor sind, sondern betrachten, „Wie“ diese Ansprüche auf typische Weise im Labor umgesetzt werden. Wenn die Ebene der Außenstruktur vereinfacht betrachtet ,Warum-Fragen" thematisiert, so widmen sich die beiden anderen Ebenen den, Wie-Fragen ' der Umsetzung, die sich aus diesen Prinzipien und Ansprüchen ergeben. Aus diesem Grund können die beiden letztgenannten Analyseebenen als die Ethnomethoden der Handelnden betrachtet werden. Diese Ethnomethoden verhelfen dazu, auf eine tradierte und phänomenspezifische Art und Weise die typische Ordnung des Phänomens des Laborexperiments im ökonomischen Laboratop hervorzubringen.

\subsubsection{Akteurskonzept und Rationalitätsverständnis}

Die nachfolgende Darstellung begrenzt sich auf die ökonomische Perspektive auf Rationalität und die daran anknüpfenden Akteursmodelle. Auf eine differenzierte 
Auseinandersetzung mit der soziologischen Betrachtung und Verwendung des Rationalitätsbegriffs und den auf diesem fußenden Akteursmodellen wird dabei bewusst verzichtet. Der Grund dieser Fokussierung besteht darin, dass die Zielstellung der vorliegenden Arbeit nicht darin liegt, allgemeine Aussagen über Rationalität und rationales Handeln zu produzieren. Vielmehr soll im Prozess der Arbeit empirisch und datengeleitet herausgearbeitet werden, wie das ökonomische Laborexperiment durch typische Muster kommunikativer Handlungen in seinem Vollzug ein Umfeld für Handlungen schafft, die von den Forschenden als rationales Entscheiden interpretiert werden können. Es gilt somit im Abschnitt der Außenstruktur die zentralen Konzepte und Feldbegriffe zu rekonstruieren, an welchen sich die Forschenden in ihren Handlungspraktiken orientieren.

Wie oben ausgeführt besteht eine grundlegende Annahme der Ökonomie in der Rationalität der Akteure. Im Folgenden soll diese Annahme wie auch das damit verbundene Konzept des Homo oeconomicus und der begrenzten Rationalität (Simon 1955, 1956, 1959) detaillierter dargestellt werden, um Hintergründe und Zusammenhänge dieser Position zu beleuchten. Denn als zentrale Prämissen haben diese beiden Konzepte Auswirkungen auf die empirische Forschung im Labor. Und auch wenn das Konzept des klassischen Homo oeconomicus, wie sich im Folgenden zeigen wird, in seiner ursprünglichen ,Strenge ' durch die Arbeiten von Herbert A. Simon und die Durchsetzung seines Konzepts der ,begrenzten Rationalität“ aufgebrochen wurde, wird von der grundsätzlichen Prämisse der handlungsleitenden Rationalität der Akteure weiterhin nicht abgewichen. Wie die Ausführungen zeigen werden, wird am Rationalitätsideal als zentraler Grundannahme weiterhin festgehalten, auch wenn sich die Argumentationsgrundlage gewandelt hat.

\subsubsection{Homo oeconomicus}

Das Konzept des ,Homo oeconomicus' ist ein klassisches theoretisches Modell der Wirtschaftswissenschaft und zugleich der Spieltheorie. Im Zusammenhang mit den Ausführungen zur Spieltheorie und zu von Neumann (Abschnitt 2.2.1) wurde bereits kurz auf das Konzept verwiesen. Grundlegend beschreibt dieses Modell Akteure als Nutzenmaximierer. Der handelnde Akteur wird dabei durch eine feste Ordnung seiner Präferenzen und durch die Kenntnis seiner Restriktionen charakterisiert. In seinen Handlungsentscheidungen folgt er bei der Wahl zwischen alternativen Handlungen stets seinen eigenen egoistischen Zielen. Die Ordnung seiner Präferenzen und die damit einhergehende Entscheidung für eine Handlungsalternative basiert dabei auf der Beurteilung des Nutzens, den die Handlungsalternativen für ihn bieten. Er verfügt zudem über vollständige Informationen über alle Handlungsalternativen und die sich aus ihnen ergebenden Konsequenzen und bezieht sie in seine Bewertung des Nutzens ein. Verbunden 
damit ist die Annahme, dass es für den Handelnden stets rational ist, seinen eigenen egoistischen Nutzen zu maximieren. Rationale Akteure wählen in der Konsequenz die Handlungsalternative, die den größten Nutzen verspricht und sind somit Nutzenmaximierer. Es handelt sich bei dem Homo oeconomicus somit um das Modell eines Akteurs, der dem Rationalitätsprinzip folgt und damit ein uneingeschränkt rationales Verhalten bei der Wahl seiner Handlungsentscheidungen aufweist (vgl. Franz 2004; Rolle 2005; Kirchgässner 2008; Rost 2008).

Über die genauen Ursprünge des Homo oeconomicus als Leitbilds des nutzenmaximierenden Wirtschaftsmenschen besteht eine gewisse Uneinigkeit. Eine deutliche Inspiration wird jedoch Isaac Newtons mechanistischem Weltbild, den Ideen von Thomas Hobbes über den Konkurrenzkampf als menschlichem Naturzustand sowie Adam Smiths Ansicht zugesprochen, dass Menschen zu ihrem eigenen Nutzen handeln (Rost 2008; Rolle 2005). Der Utilitarismus nach Stuart Mill und Jeremy Bentham bildet mit seinen zentralen Annahmen des hedonistischen Prinzips, des ethischen Egoismus, der Vorstellung der Interessenharmonie und seiner empirische Orientierung eine entsprechende Grundlage für die Herausbildung dieses Modellvorstellung (Rolle 2005: 114 f.). ${ }^{6}$ Aus Sicht von Bentham (1748-1832) und Stuart Mill, zwei der zentralen Gründerfigur des Utilitarismus, sind die Menschen stets durch zwei verschiedene Aspekte determiniert: „Nature has placed mankind under the governance of two sovereign masters, pain and pleasure" (Bentham 1996: 11). Das Spannungsverhältnis von Lust (pleasure) und Unlust (pain) bestimmt als positive und negative Sanktionen das Handeln der Menschen. Hedonistischen Interessen folgend, versuchen Menschen durch ihr Handeln ihre Interessen bestmöglich und mit den geringstmöglichen Opfern zu verfolgen. In der Maximierung von Lust und der Minimierung von Unlust sieht die utilitaristische Schule einen objektiven Maßstab für die Messung von Wünschen und Abneigungen des handelnden Menschen. Nutzen gilt nun als das quantifizierbare Maß für Lust oder Unlust. Nach Bentham variieren Lust- und Unlustempfindungen dabei hinsichtlich ihrer Ausprägung in vier Dimensionen: Dauerhaftigkeit, Intensität, Eintrittswahrscheinlichkeit und zeitlicher Abstand zueinander (vgl. Rolle 2005: 119 f.). Benthams Perspektive folgend handelt der Mensch rational, wenn er zwischen verschiedenen Alternativen abwägt und diejenige mit dem größten Nutzen wählt. ${ }^{7}$

\footnotetext{
${ }^{6}$ Eine ausführliche Darstellung der wissenschaftsanthropologischen Auseinandersetzung mit der philosophischen Entwicklungsgeschichte des Konzepts des Homo oeconomicus liefert Rolle (2005) in seiner Abhandlung „Homo Oeconomicus, Wirtschaftsanthropologie in philosophischer Perspektive“.

${ }^{7}$ Gesellschaftliche und individuelle Bestrebungen der Nutzenmaximierung werden dabei nicht als gegensätzlich betrachtet. Utilitaristisch betrachtet ist ein radikaler Egoismus, der im Sinne Hobbes dem gesellschaftlichen Allgemeinwohl entgegensteht, vielmehr eine Verfehlung der
} 
Gängige Kritik am Konzept des Homo oeconomicus ist die Differenz zwischen der idealtypischen Modellvorstellung und den empirisch beobachtbaren Entscheidungen von Akteuren. Aus diesem Grund muss deutlich darauf verwiesen werden, dass auch den ökonomischen ForscherInnen durchaus bewusst ist, dass es sich um ein Modell menschlichen Verhaltens handelt und nicht um die Beschreibung eines Menschen. Fritz Machlup schlug in diesem Sinne vor, den Begriff „Homunculus oeconomicus“ (Machlup 1960: 42; Machlup 1978: 114) zu verwenden, um darauf $\mathrm{zu}$ verweisen, dass dieses Modell ein von Menschen gemachtes ist. Mittels dieses Modells werden zentrale Prämissen der ökonomischen Forschung gebündelt, die als unhinterfragte Grundannahmen in die Konzeption von wissenschaftlichen Modellen und Theorien Eingang finden. In der ökonomischen Forschung fungiert das Modell des Homo oeconomicus als idealtypische Hintergrundfolie. Die Forschenden sind sich jedoch dessen bewusst, dass damit dem realweltlichen Handeln von Akteuren nur in sehr eingeschränktem Maß entsprochen wird. Der Ursprung des Models des Homo oeconomicus ergibt sich aus dem Erkenntnisinteresse der Ökonomik, welches sich mit den Konsequenzen menschlichen Handelns befasst und nicht mit dem Handeln selbst.

Die Differenz zwischen der klassischen Form des nutzenmaximierenden Homo oeconomicus und der Prämisse in der spieltheoretischen Nutzung von Neumann und Morgenstern besteht darin, dass in der spieltheoretischen Konzeption nur von dem ,erwarteten Nutzen' bei der Wahl einzelner Handlungsalternativen ausgegangen werden kann, da das Verhalten des Gegenspielers nie eindeutig vorhersehbar ist. ${ }^{8}$ Gerade in komplexen Entscheidungssituationen zeigte jedoch das Modell des Homo oeconomicus seine begrenzte Anwendbarkeit für die modellhafte Analyse empirischer Phänomene und wurde so zum Ausgangspunkt für Weiterentwicklungen.

individuellen Nutzenmaximierung. Denn auf die gesamte Lebenszeit hin betrachtet kann individueller Egoismus und die Ausrichtung auf Eigennutzen nicht zur Nutzenmaximierung führen, sondern bestenfalls auf eine kurzfristige Lustempfindung abzielen. Relevant ist hier somit, auf welche Zeitspanne sich die Beurteilung der Nutzenmaximierung der Handlungen richtet. Was kurzfristig als nutzenmaximierende Handlung erscheint, kann langfristig betrachtet auf die ,existenziale Nutzenfunktion“ bezogen (Rolle 2005: 120) weit weniger rational sein (vgl. Rolle 2005: 119 f.).

${ }^{8}$ Im Zuge der Unterscheidung von klassischer Entscheidungstheorie und der Spieltheorie als interaktiver Entscheidungstheorie wurde auf diese Differenz bereits im Abschnitt 2.2.2 verwiesen. 


\title{
5.2.1.2 Herbert Simon und die begrenzte Rationalität
}

Eine wichtige Weiterentwicklung von dem uneingeschränkt rationalen Homo oeconomicus hin zu einem Modell, welches zur Prognose und Erklärung empirischer Ergebnisse genutzt werden kann, stellt die Arbeiten Herbert A. Simon dar. Empirischer Anstoß war, laut Smith (1992) ${ }^{9}$, die Diskussion auf der Konferenz „The Design of Experiments in Decision Processes“ in Santa Monica. Simon diskutierte hier mit den anwesenden Psychologen und Ökonomen ein Experiment des Psychologen William Estes und dessen Ergebnisse. In diesem Experiment sollten Probanden vorhersagen, welche von zwei gegebenen Lampen als nächste aufleuchten würde. Das Aufleuchten der Lampen war jedoch zufällig und folgte keinem vorhersehbaren Muster. Während Psychologen das Verhalten der Probanden, ihren Lerntheorien folgend, als rational erachten, war das Verhalten aus Sicht von Entscheidungs- und Spieltheoretikern irrational. Es zeigte sich, dass die Differenz des Rationalitätsverständnisses dabei maßgeblich auf dem unterschiedlichen Informationsstand von ExperimentatorInnen und ProbandInnen und der daraus resultierenden Bewertung der Entscheidungsalternativen gründete.

\begin{abstract}
"Perhaps the most useful lesson to be learned from the derivation is the necessity for careful distinctions between subjective rationality (i.e., behavior that is rational, given the perceptual and evaluation premises of the subject), and objective rationality (behavior that is rational as viewed by the experimenter). Because this distinction has seldom been made explicitly by economists and statisticians in their formulations of the problem of rational choice, considerable caution must be exercised in employing those formulations in the explanation of observed behavior. [...] If we accept the proposition that organismic behavior may be subjectively rational but is unlikely to be objectively rational in a complex world then the postulate of rationality loses much of its power for predicting behavior. To predict how economic man will behave we need to know not only that he is rational, but also how he perceives the world-what alternatives he sees, and what consequences he attached to them [...] [Simon 1955]. We should not jump to the conclusion, however, that we can therefore get along without the concept of rationality." (Simon 1956, 271-272, zit. nach Smith 1992: 263)
\end{abstract}

Auf diesen Erkenntnissen gründete Simon die Differenz seiner Konzepte von formaler Rationalität (,substantive rationality“) und prozeduraler Rationalität (,procedural rationality“) bzw. objektiver und subjektiver Rationalität und schaffte damit die zentrale Grundlage seiner Theorie der ,begrenzten Rationalität' (,Bounded rationality“) (Smith 1992: 263; Simon 1955, 1956, 1957, 1959, 1976, 2013

\footnotetext{
${ }^{9}$ Wie weiter oben in den Ausführungen zur Etablierung der Spieltheorie dargestellt, nahm Herbert Simon auch an der von Smith organisierten Konferenz in Santa Monica teil (siehe auch Abschnitt 2.2.2).
} 
[1945]). Die Grundgedanken des Konzepts begrenzter Rationalität lassen sich wie folgt zusammenfassen (vgl. Simon 1955, 1957, 1959).

Nach Simon sind Menschen bei der Bewertung von Handlungsalternativen im Zuge der Entscheidungsfindung häufig nicht zu uneingeschränkter Rationalität fähig. Die zentralen Gründe dafür sieht Simon in der kognitiven Beschränkung der Handelnden und in der Anwendung von Heuristiken. Kognitive Einschränkungen würden dabei bedingen, dass den Handelnden nicht alle notwendigen Informationen zur optimalen rationalen Beurteilung ihrer Handlungsalternativen zugänglich sind. Der Grund hierfür liegt in den begrenzten Kapazitäten der Akteure hinsichtlich der Aufnahme und Verarbeitung der beurteilungsrelevanten Informationen. So können Handelnde beispielsweise nicht alle Handlungsalternativen bei ihrer Bewertung in Betracht ziehen, da ihnen möglicherweise die Kenntnis über einzelne Alternativen völlig fehlt. Die Bewertung des Nutzens der Alternativen kann zudem dadurch verzerrt werden, dass vom Standpunkt der Akteure nicht immer ohne weiteres ersichtlich ist, welche Konsequenzen einzelne Alternativen besitzen. Die statistische Beurteilung der besten Handlungsalternative kann in komplexen Situationen auch die mathematischen Fähigkeiten der Handelnden überschreiten. Unterschiedliche Informationsstände führen $\mathrm{zu}$ unterschiedlichen Bewertungen der Entscheidungssituation und der rationalen Wahl zwischen Entscheidungsalternativen, wie auch das oben rezipierte Experiment Estes zeigte. Simon schlussfolgerte nun, dass Handelnde aufgrund dieser Beschränkungen der Informationsverarbeitung und -verfügbarkeit gerade in komplexen Entscheidungssituationen auf Heuristiken zurückgreifen und einfache Entscheidungsregeln nutzen. Dies ermöglicht es ihnen, die Zeit und den Aufwand für die Informationszusammenstellung und -bewertung in diesen Situationen deutlich zu reduzieren. Handelnde tun dies auch auf die Gefahr hin, dass diese auf Heuristiken aufbauende Bewertung der Handlungsalternativen im Sinne der Nutzenmaximierung fehlerhaft ist. Damit wird den Handelnden nicht abgesprochen, dass sie rational handeln und versuchen, ihren Nutzen zu maximieren. Im Unterschied zum „economic man“10 entscheiden die Handelnden jedoch unter den oben genannten Einschränkungen (von Zeit und Ressourcen) und suchen aus diesem Grund

\footnotetext{
${ }^{10}$ Simon veröffentlichte bereits seit den 1940er Jahren Arbeiten, in denen er sich kritisch mit dem ökonomischen Modell des allwissenden und nutzenmaximierenden ökonomischen Entscheiders ("economic man") auseinandersetzte. Empirischer Gegenstand seiner Reflexion war dabei häufig das Entscheidungsverhalten von Verwaltungsbeamten ("administrative man" oder "administrator"), die bei ihren Entscheidungen eben diesen Ansprüchen nicht genügen können und dies auch nicht müssen. ,Whereas economic man supposedly maximizes - selects the best alternative from all those available to him, his cousin, administrative man, satisfices - looks for a course of action that is satisfactory or 'good enough' [...] Economic man purports to deal with the 'real world' in all its complexity. The administrator recognizes that
} 
nach einer befriedigenden Lösung (satisficing), anstatt einer optimalen Lösung (maximizing) für ihr Entscheidungsproblem (Simon 1959: 262 f.).

In der Konzeption von ökonomischen Laborexperimenten und der Bildung zu testender Hypothesen wird allerdings nicht von ganzheitlichen Personen ausgegangen, sondern von ökonomischen Agenten mit vorspezifizierten Merkmalen (Friedman \& Sunder 1994: 13). Wie bereits dargestellt, zielen ökonomische Laborexperimente auf die Hervorbringung eines spezifischen Entscheidungsumfeldes ab. Es handelt es sich hierbei um abstrakte mikroökonomische Umwelten, die durch ökonomische Agenten und eine ökonomische Institution spezifiziert werden. Die Agenten werden dabei durch die ökonomisch relevanten Charakteristika und somit durch ihre Präferenzen, Informationen und Ressourcen definiert (ebd.). Institutionen bezeichnen hier das in dieser Umwelt vorliegende Set von Handlungsmöglichkeiten eines Agenten und die Konsequenzen, die sich aus Handlungswahlen und Kombinationen eben dieser ergeben. Es kann sich dabei beispielsweise um Käufer und Verkäufer auf einer bestimmten Art von Markt handeln, welcher durch spezifische Regeln des Tauschs bestimmt ist. Auch wenn anerkannt wird, das die menschlichen Forschungssubjekte spezifische idiosynkratische Prägungen und Charakteristika besitzen, zielen Experimente zumeist darauf ab, dass ProbandInnen die vorspezifizierten Merkmale annehmen und das Set zur Verfügung stehender Handlungsoptionen antizipieren (ebd.). Im Zuge von ökonomischen Laborexperimenten wird gezielt auf bestimmte Verfahren zurückgegriffen, um eben diese ökonomischen Umwelten hervorzubringen und ökonomische Agenten zur Übernahme vorspezifizierter Merkmale zu motivieren. Eingang in die Konzeption von Experimenten fand Simons Konzept der begrenzten Rationalität maßgeblich durch die Experimente der Psychologen Daniel Kahneman und Amos Tversky, die durch verschiedene experimentelle Studien

the world he perceives is a drastically simplified model of the buzzing, blooming confusion that constitutes the real world. The administrator treats situations as only loosely connected with each other - most of the facts of the real world have no great relevance of any single situation and the most significant chains of causes and consequences are short and simple [...] Administrators and everyone else, for that matter take into account just a few of the factors of the situation regarded most relevant and crucial [...] Because administrators satisfice rather than maximize, they can choose without first examining all possible behavior alternatives and without ascertaining that these are in fact all alternatives. Because they treat the world as rather empty and ignore the interrelatedness of all things (so stupedifying to thought and action), they can make their decisions with relatively simple rules of thump that do not make impossible demands upon his capacity for thought. Simplification may lead to error, but there is no realistic alternative in the face of the limits on human knowledge and reasoning" (Simon 2013 [1945]: 119). 
nachwiesen, dass sich Versuchspersonen in ihren Entscheidungen begrenzt rational verhalten. Die Reflexion der Simonschen Erkenntnisse über die Differenz von subjektiver und objektiver Rationalität - inspiriert durch die Arbeiten von Sidney Siegel - und ihre Aufnahme durch Vernon Smith führte auf der Ebene der Methodologie der experimentellen Wirtschaftswissenschaften zur Begründung zentraler Paradigmen, wie der ,induzierten Werttheorie“.

\subsubsection{Grundlegende methodische Prinzipien der experimentellen Wirtschaftsforschung}

Die nachfolgenden Ausführungen zeigen auf, wie die Erkenntnisse von Herbert Simon über die begrenzte Rationalität von Akteuren in der experimentellen Wirtschaftsforschung ihren anerkennenden Niederschlag fanden und in methodische Leitlinien für die Forschung umgewandelt wurden. Als einflussreicher Orientierungspunkt in der Konzeption von wirtschaftswissenschaftlichen Experimenten gelten die Arbeiten des Forscherduos Daniel Kahneman und Amos Tversky. Sie motivierten einerseits vielfältige empirische Studien, haben darüber hinaus jedoch zu einer verstärkten Aufmerksamkeit gegenüber unbeabsichtigten Framing-Effekten ${ }^{11}$ in der Durchführung und Konzeption von Experimenten geführt. Die ,induzierte Werttheorie“ von Smith (1976) stellte hingegen die zentrale Grundlagen für die praktische Durchführung von Experimenten in der experimentellen Wirtschaftsforschung und bildet somit den zentralen Referenzpunkt für die Nutzung monetärer Incentives in Experimenten. Mit ihr wird der Anspruch verbunden, ProbandInnen so zur Einnahme vorspezifizierter Merkmale motivieren zu können.

\subsubsection{Kahneman und Tversky und ihr Konzept des Framings}

Simons Konzept der begrenzten Rationalität fußt auf der Annahme, dass Handelnde bei der Lösung von Entscheidungsproblemen nicht nach optimalen Lösungen im Sinne der Nutzenmaximierung suchen. Aufgrund beschränkter Kompetenzen der Informationsverarbeitung und -verfügbarkeit suchen sie stattdessen - unter Zuhilfenahme von Heuristiken und simplen Entscheidungsregeln

\footnotetext{
${ }^{11}$ Der Begriff wird hier nicht im soziologischen Sinne von Goffman genutzt, sondern im Sinne der gängigen Rezeption der experimentellen Forschenden im Bereich der (Verhaltens-) Ökonomie. Trotz der rein sprachlichen Übersetzbarkeit von „Framing“ und „Rahmung“ wird hier der englische Begriff des Frames bzw. Framing beibehalten, da dies im Sinne eines Feldbegriffes der üblichen Verwendung entspricht.
} 
- nach befriedigenden Lösungen (satisficing) für komplexe Entscheidungssituationen (Simon 1959: 262 f.). Die Psychologen Daniel Kahneman und Amos Tversky belegten durch ihre experimentellen Studien die Relevanz des Simonschen Konzepts für die Erklärung des Entscheidungsverhaltens, welches ProbandInnen in Experimenten zeigten. Für seine Arbeiten, die zur Aufnahme psychologischer Aspekte in die wirtschaftswissenschaftliche Forschung führten, wurde Kahneman 2002 mit dem Wirtschafts-Nobelpreis geehrt. Wie der nachfolgende Ausschnitt aus einem Gespräch mit einer ökonomischen Experimentalforscherin zeigt, gelten die Arbeiten des Forscherduos als zentrale Inspiration für die Aufnahme psychologischer Aspekte in die ökonomische Forschung:

„Also Kahneman und Tversky sind sozusagen die Begründer von ,Psychology in economics' aber auch jetzt weniger methodisch, weil die mit ihren Methoden, weil die mit ihren Methoden noch sehr nah an der Psychologie waren. Die haben ja meistens, ja häufig auch hypothetische Fragen gestellt und die Leute nicht bezahlt. Aber die haben die ganzen Fragen aufgeworfen eigentlich [...] Für den bounded rationality Teil sind Kahneman und Tversky sozusagen die Gründerväter. “ (Auszug aus ExpertInneninterview1)

Die durch die experimentellen Studien und die Texte von Kahneman und Tversky aufgeworfenen Fragen bildeten dabei einen zentralen Anstoß für die Beschäftigung mit psychologischen Aspekten der Entscheidungsfindung in der Ökonomie und ihrer experimentellen Beforschung. Ihre Arbeiten legten somit einen wichtigen Grundstein für die Ausbildung des Forschungszweiges der experimentellen Verhaltensökonomie, welche eine Integration psychologischer Aspekte zur Validitätssteigerung ökonomischen Entscheidungsmodelle anstrebt. Wie im Gesprächsausschnitt oben angemerkt, verfolgt die Umsetzung der Untersuchung dieser Aspekte in der ökonomischen Forschung jedoch anderen Vorgaben und Standards.

Ein von Kahneman und Tyersky beforschter Aspekt, der sich in der Durchführung und Konzeption von Experimenten als folgenreich erweist, sind ihre Arbeiten zum Framing der in Experimenten dargestellten Spiel- bzw. Entscheidungssituationen. Eine grundlegende Annahme der ökonomischen Nutzentheorie ist die Beschreibungsinvarianz. Diese geht davon aus, dass die rein semantische Darstellung einer Entscheidungssituation keinen Einfluss auf die Entscheidungspräferenzen der jeweiligen Personen haben dürfte, wenn die Eintrittswahrscheinlichkeiten 
der Ergebnisse gleich bleiben. Eine Person sollte demnach die gleiche Entscheidung fällen, auch wenn sich die Darstellung der Entscheidungssituation ändert. Im Anschluss an die Psychologen Kahneman und Tversky erkennt man jedoch in der Verhaltensökonomie an, dass die Semantik durchaus einen deutlichen Einfluss auf die Entscheidungsfindung der ProbandInnen haben kann (Camerer 2003). Kahneman und Tversky (1981) zeigten in ihren Experimenten zum „AsianDisease-Problem"12, dass signifikante Unterschiede in den Entscheidungen der ProbandInnen auf unterschiedliche Formulierungen der Entscheidungssituation zurückgeführt werden können.

Der verhaltensökonomische Begriff des Framing fußt auf diesen Erkenntnissen von Kahneman und Tversky. Dieser Framing-Begriff geht dementsprechend davon aus, dass die Entscheidungssituation für die TeilnehmerInnen in einem Experiment maßgeblich durch die sprachlichen Formulierungen in den schriftlichen Instruktionen bestimmt wird. Framing-Effekte im allgemeinen wirtschaftswissenschaftlichen Sinne bezeichnen Reaktionen auf eine - hinsichtlich des Nutzenwertes der Wahloptionen - irrelevante Veränderung der Versuchsanordnung. Aus diesem Grund werden Framing-Effekte auch als Darstellungseffekte bezeichnet. Levin et al. (1998) verweisen darauf, dass bezüglich des Aspekts

\footnotetext{
${ }^{12}$ Kahneman und Tversky (1981) zeigten, dass signifikante Unterschiede in den Entscheidungen der ProbandInnen auf unterschiedliche Formulierungen der Entscheidungssituation, etwa auf den Unterschied in der Verwendung von Begriffen wie „Sterblichkeit“ und „Überleben“, zurückgeführt werden können. Auf diese Studie wird üblicherweise unter dem Begriff des „Asian-Disease-Problem“ rekurriert. Es wurden dabei zwei Serien von Experimenten durchgeführt, in denen ein gleicher Sachverhalt unterschiedlich formuliert wurde. Die Entscheidungssituation wurde wie folgt dargestellt: Die Vereinigten Staaten bereiten sich auf den Ausbruch einer ungewöhnlichen asiatischen Krankheit vor, bei der 600 Personen sterben könnten. Den Teilnehmern des Experiments bieten sich jeweils zwei mögliche Behandlungsalternativen. In der ersten Variante des Experiments bot Programm A die Möglichkeit, 200 Personen zu retten. Programm B hätte zur Folge, dass mit einer Wahrscheinlichkeit von 1/3 alle Menschen gerettet werden würden und mit einer 2/3 Wahrscheinlichkeit niemand gerettet werden würde. 72 Prozent der Befragten entschieden sich in diesem Fall für Programm A und nur 28 Prozent für Programm B. Eine andere Gruppe von ProbandInnen sollte, basierend auf dem gleichen Ausgangsproblem, zwischen den Optionen C und D wählen. Der Einsatz von Programm C hätte zur Folge, dass 400 Menschen sterben würden. Bei Programm D bestand eine Wahrscheinlichkeit von $1 / 3$ dass alle 600 Menschen gerettet werden würde und eine $2 / 3$ Wahrscheinlichkeit, dass niemand gerettet werden würde. In diesem Experiment entschieden sich 78 Prozent der Befragten für Programm D. Kahneman und Tversky (1981) verwiesen nun darauf, dass ein Vergleich der Alternativen A und C bzw. B und D zwar zu gleichen quantitativen Ergebnissen führte, die Entscheidung der Teilnehmer jedoch durch die unterschiedlichen sprachlichen Formulierungen der Entscheidungssituation so stark beeinflusst wurden. Eben diese Differenz im Entscheidungsverhalten, die sich durch die Darstellung der Entscheidungssituation ergibt, wird als Framing bezeichnet.
} 
des Framings in Entscheidungssituationen drei unterschiedliche Manipulationsarten vorliegen können: Wahloptionen können unterschiedlich riskant sein (risky choice Framing), unterschiedliche Attribute von Wahlalternativen betonen (attribut framing) oder das Ziel, welches durch Handlungsentscheidungen angestrebt wird, verschiedentlich darstellen (goal framing). Gemeinsam ist diesen FramingEffekten jedoch, dass sie als etwas betrachtet werden, das durch die Darstellung der Entscheidungssituationen hervorgebracht wurde.

In der Konzeption der Experimente wird den schriftlichen Instruktionen, die die Entscheidungssituationen für die ProbandInnen darstellen sollen, aus diesem Grund ein besonders hoher Stellenwert beigemessen. Instruktionen bilden ein wichtiges binnenstrukturelles Merkmal (Abschnitt 5.3.4.). Da die Instruktionen von den WissenschaftlerInnen ausgearbeitet und an die TeilnehmerInnen verteilt werden, gehen die Forschenden davon aus, dass sie eine Kontrolle über die Darstellung der Situation im Labor besitzen. Das Framing wird als etwas Gesetztes verstanden, das von den ökonomischen WissenschaftlerInnen aktiv gesteuert werden kann. Dies erfolgt, indem sie gezielt spezifische Aspekte der Instruktionen oder des Set-up variieren. Eine Veränderung des Framing wird meist durch eine Variation der schriftlichen Instruktionen hervorgebracht. Bestimmte Begriffe sollen auf eine spezifische Art und Weise verstanden werden und es erlauben, eine gezielte Informationsgrundlage für die nachfolgenden Entscheidungssituationen und Verhaltensspiele zu schaffen. Wie in den nachfolgenden Abschnitten der Analyse noch weiter ausgeführt wird, fördern spezifische Techniken der Forschenden, dass die ProbandInnen diese Informationsgrundlage in der antizipierten Weise verstehen. Der methodologischen Prämisse folgend, dass die Forschenden fähig sind, im Verlauf der Laborexperimente die weiteren Randbedingungen konstant zu halten, bilden die Instruktionen einen entscheidenden Schritt in der Induzierung der Rahmung der Laborsituation.

\subsubsection{Vernon Smith und die induzierte Werttheorie}

Wie zu Beginn der Arbeit in der Feldbeschreibung der experimentellen Wirtschaftsforschung dargestellt, ist Vernon Smith eine zentrale Schlüsselfigur in der Etablierung und Reflexion methodischer Standards in der experimentellen Wirtschaftsforschung (Abschnitt 2.2.2). Zusammen mit Daniel Kahneman wurde er 2002 für die Einführung der Laborexperimente als wissenschaftliche Methode in der mikroökonomischen Forschung mit dem Wirtschafts-Nobelpreis geehrt. Bereits in seinen ersten Experimenten stellte Smith fest, welchen hohen Stellenwert das Design von Experimenten auf ihre Ergebnisse hat. Die Begründung für den Einsatz von monetären Anreizen, die als Grundpfeiler von ökonomischen 
Experimenten gelten, basieren dabei auf Smith induzierter Werttheorie (Smith 1976).

Smith argumentiert hier, dass man durch die Schaffung eines geeigneten Anreizsystems die Motive der TeilnehmerInnen im Experiment kontrollieren kann und so in der Lage ist, die ProbandInnen dazu zu bewegen, bestimmte vorspezifizierte Merkmale anzunehmen. Smith folgend, müssen für das Wirken des genutzten Anreizsystems drei Grundbedingungen erfüllt sein.

Die erste Bedingung ist die Monotonie des Nutzens: Die TeilnehmerInnen müssen stets ein Interesse daran haben, mehr von der in Aussicht gestellten Belohnung zu erhalten. Es darf keine Sättigung dieses Bedürfnisses auftreten, da ansonsten der Anreizmechanismus seine Wirkung verlieren würde. Die experimentellen WirtschaftswissenschaftlerInnen sind sich (weitgehend) darüber einig, dass diese Bedingung durch den Anreizmechanismus der monetären Belohnung in Laborexperimenten am besten erfüllt werden kann, wie beispielsweise das Zitat von Camerer (2003: 39) belegt: ,[B]y inducing value using money payments, the experimenter need to rely only on the assumption that everybody likes having more money and nobody gets tired of having more of it". 13

Die zweite Bedingung ist die Salienz. Sie beschreibt, dass die Handlungen der Teilnehmer auszahlungsrelevant sein müssen. Die Bezahlung der Teilnehmer erfolgt demnach entscheidungsabhängig. Es wird entsprechend kein fixer Betrag festgelegt, den alle Versuchspersonen am Ende des Experiments erhalten. Das Handeln und Entscheiden der ProbandInnen im Verlauf der Datenerhebung ist vielmehr ausschlaggebend. Alle datenerhebungsrelevanten Entscheidungen sollen dabei incentiviert sein.

Eine dritte Bedingung ist die Dominanz. Die Belohnung im Experiment muss alle anderen subjektiven Kosten der Teilnehmenden wie Hunger, Langeweile oder soziale Erwünschtheitseffekte gegenüber den Forschenden dominieren können. Die monetäre Motivation wird bewusst eingesetzt, um andere Reize zu überlagern und somit um potenzielle Störfaktoren auszuschalten oder zumindest zu minimieren.

Die Hervorbringung der interessierenden mikroökonomischen Umwelten - als Konstellation von in ihr agierenden ökonomischen Agenten und einem Set von Handlungsmöglichkeiten und Konsequenzen - soll so ermöglicht und befördert werden. Ganz im Sinne von Smith (1976) wird davon ausgegangen, dass man

\footnotetext{
${ }^{13}$ Im Anschluss an die oben zitierte Aussage fügt Camerer (2003) in seinem breit rezipierten Standardwerk zur verhaltensökonomischen Spieltheorie eine (wohl amüsant gemeinte) Bemerkung hinzu, die die Allgemeingültigkeit der Wirkung monetärer Incentives unterstreichen soll: „If you know anybody who is tired of getting more money let me know; I'll take their leftovers!“ (Camerer 2003, S. 39).
} 
durch die extrinsische Motivation des Geldanreizes eine bessere Kontrolle über die Handlungsmotivation der ProbandInnen hat. Smiths (1992) Diskussion und Auseinandersetzung mit Simons Ideen und Konzepten von objektiver und subjektiver Rationalität bzw. der begrenzten Rationalität von Versuchspersonen (siehe oben) bilden einen wichtigen Ausgangspunkt für seine Prinzipien des Experiments. Durch die Nutzung eines dauerhaften, dominanten und salienten Anreizes in der Durchführung von Experimenten wird somit befördert, dass die ,subjektive “ Rationalität der ProbandInnen der ,objektiven" Rationalität des Versuchsleiters angenähert wird.

\subsubsection{Das institutionelle Setting}

Einen Teil der institutionellen Außenstruktur des ökonomischen Laboratops bilden das übliche soziale Setting und die sozialen Rollen oder Rollentypen, die an der Erzeugung dieses Phänomens beteiligt sind. Neben der Klärung grundlegender epistemischer Aspekte der experimentellen Wirtschaftsforschung werden aus diesem Grund auch weitere außenstrukturelle Rahmungsfaktoren dargestellt. Zugleich handelt es sich bei diesen um Grundmomente der sozialen Veranstaltungen und des Rahmenbildungsprozesses, der in dieser Arbeit betrachtet wird.

Wirtschaftswissenschaftliche Laborexperimente werden vorrangig an Universitäten durchgeführt. Auch im von mir beobachteten Fall befand sich der Laborraum in einer Universität: Spezifischer lag das Experimentallabor in den Räumlichkeiten des Fachgebiets für experimentelle Wirtschaftsforschung der Technischen Universität Berlin. Hinsichtlich der Rahmung und Charakterisierung der sozialen Situation des Laborexperiments ist dies von großer Bedeutung. Für die Teilnehmenden wird auf diese Weise deutlich markiert, dass es sich bei den durchgeführten Experimenten um Datenerhebungen zum Zweck wissenschaftlicher Forschung handelt. Zugleich müssen auch das Labor selbst und die darin erfolgenden Datenerhebungen den wissenschaftlichen und bürokratischen Standards entsprechen, die diese institutionelle Einbettung erforderlich macht.

\subsubsection{Soziale Rollen im Laborexperiment}

Das Phänomen des ökonomischen Laborexperiments und die darin Handelnden treten hier als Teil des Wissenschaftsmilieus in Erscheinung. Die sozialen Rollen, die den Handelnden zugeschriebenen werden, sind aus diesem Grund die der Forschenden oder ExperimentatorInnen und die der beforschten ProbandInnen oder VersuchsteilnehmerInnen. Im Anschluss an die Ethnomethodologie und 
das dort ausgeführte Konzept der situierten Akteure sowie unter Berücksichtigung der relationalen Perspektive des kommunikativen Konstruktivismus geht es in der nachfolgenden empirischen Analyse insbesondere um die Anerkennung des Konstruktionsaspekts und die beobachtbare Hervorbringung dieser Rollen sowie der mit diesen verbundenen typisierten Handlungszuschreibungen. Im Abschnitt der Außenstruktur werden jedoch einführend die Rollenkonzepte beschrieben, welche den Prozess des Laborexperiments aus einer ,Außenperspektive' kennzeichnen.

\section{ProbandInnen}

Die Konzeption der Rolle der ProbandInnen hängt maßgeblich vom grundlegenden Akteurskonzept ab, welches im Labor seine Anwendung findet. Grundlegend wird davon ausgegangen, dass die ProbandInnen als normorientierte und nutzenmaximierende Akteure konzipiert werden können, die bestimmte Ziele verfolgen und sich an vorgegebenen Regeln orientieren (Berger 2015). Im Anschluss an Simons „bounded rationality“ erkennen die Laborforschenden an, dass hinsichtlich der Rationalität der Entscheidungen der ProbandInnen bestimmte Begrenzungen vorliegen, die zu Abweichungen und Anomalien führen können.

Durch die Nutzung monetärer Anreize ist es möglich, bestimmte Handlungsmotive und -alternativen zu überlagern und für die Teilnehmenden unattraktiver zu machen (Smith 1976). Die Aufgabe der ProbandInnen wird von den Forschenden darin gesehen, sich mit den an sie gestellten Aufgaben und Spielen auseinanderzusetzen. Bei der Bearbeitung der Aufgaben sollen sie den vorgegebenen Regeln der Forschenden folgen und sich an den zur Verfügung gestellten Informationen orientieren. Da die durchgeführten Experimente im Labor dazu dienen, Daten für eine wissenschaftliche Analyse zu erheben, geht dies damit einher, dass die TeilnehmerInnen bei ihren Handlungen beobachtet werden und dies auch wissen. Da die Experimente im Labor üblicherweise an Computern stattfinden, wird von den ProbandInnen erwartet, dass sie ihre zentralen Handlungsentscheidungen oder Auswahlen in einer für das Eingabegerät verwertbaren Form entäußern. Eine gewisse Nähe dieser bewussten Beobachtungssituation zu Foucaults „Überwachen und Strafen“ (2008 [1977]), seinen Ausführungen zum Benthamschen Panoptikum, liegt hier sehr nahe. Die Teilnehmenden wissen, dass sie beobachtet werden und orientieren sich in ihren Handlungen durchaus daran.

Wie die empirischen Beispiele der Krisensituationen zeigen werden (Abschnitt 5.4.7), besteht jedoch nicht in allen Fällen ein einheitlicher Konsens darüber, welche Handlungen Regelüberschreitungen darstellen. Wichtig ist hier jedoch zu bemerken, dass, auch aus Sicht vieler ProbandInnen, in diesem Laborkontext ein spezielles Set von Normen vorliegt, dass sich durchaus von dem alltagsweltlich antizipierten unterscheiden kann. Die Modulation des Alltags, die 
hier durch den Prozess der Rahmenbildung hervorgebracht wird, erzeugt dabei eine eigene Binnenlogik der Sozialwelt des Laboratops. Maßgeblich dafür ist die Anonymisierung der Teilnehmenden untereinander. Die ProbandInnen sind füreinander anonym, sodass sie nicht wissen, welche Handlungen andere TeilnehmerInnen vollzogen haben. Egoistisches oder auf Eigennutz ausgerichtetes Handeln ist somit möglich, ohne direkte soziale Sanktion. ${ }^{14}$

Hinsichtlich der Repräsentativität der Stichprobe der Teilnehmenden an psychologischen und ökonomischen Experimenten sei auf die Studie von Heinrich et al. (2010) verwiesen. Die Autoren beschreiben hier, dass es sich bei den ProbandInnen in überdurchschnittlichem Maß um WEIRD-People (Western, Educated, Industrialized, Rich and Democratic) (Henrich et al. 2010) handelt. Auch im beobachteten Labor waren nur 2 der 150 Befragten keine StudentInnen. Da die Experimente in deutscher Sprache durchgeführt wurden, mussten die Teilnehmenden über entsprechende Sprachkompetenzen verfügen. Mehrheitlich studierten sie zudem an der Universität, an welcher sich auch das Experimentallabor befand. Der Pool der registrierten ProbandInnen umfasste zum Zeitpunkt der Datenerhebung ca. 4000 Personen. Auch wenn die Experimente, wie im nachfolgend Abschnitt zum Anwerben dargestellt, grundsätzlich offen für alle Studierende und auch andere Teilnehmende sind, zeigt sich doch, dass eine gewisse Vorbildung aus Sicht einer befragten wirtschaftswissenschaftlichen Laborforscherin (Ökonomin) als vorteilhaft angesehen wird:

Ökonomin: „An der Universität X find ich, dass wir da relativ Glück haben. Für den Zweck unserer Experimente sind es eigentlich gute Studenten, weil es sehr viele sind, die naturwissenschaftlichen oder mathematik- oder ingenieurwissenschaftlichen Background haben und deswegen gut mit Zahlen umgehen können und deswegen auch halbwegs smart sind, mit Instruktionen umzugehen, aber nicht ökonomisch vorgebildet sind und die sind sozusagen gut zu haben für Experimente, solche Leute. Weil es ist manchmal schwierig. Wir hatten eine Zeit lang

\footnotetext{
${ }^{14}$ Das soll jedoch nicht bedeuten, dass es in keinem Fall eine Sanktionsmöglichkeit gäbe. Viele Experimente machen gerade den Einfluss von Fairness und Vertrauen in Entscheidungssituation zu ihrem Inhalt. Aufgrund der Anonymität der Teilnehmenden untereinander kann die Sanktion egoistischen Verhaltens jedoch stets nur in dem vorgegebenen Eingaberahmen des Entscheidungsspiels erfolgen, vermittelt über die Computeroberfläche. Faktische Sanktion in einer körperlichen, kopräsenten Interaktionssituation wird ausgeschlossen.
} 
Theologen von der Universität $Y$, weil die theologische Fakultät nebenan war und da waren welche dabei, die haben echt ((leichtes Schmunzeln und Kopfschütteln)). Ich meine das war auch interessant (.) Aber da gab es einfach welche, die haben viel schwieriger verstanden, worum es geht. Ja, oder fanden es irgendwie komisch.

Juliane: $\quad$ Die haben sich gestoßen an dem Experiment als solchem oder wie?

Ökonomin: Ja, häufig gab es auch Verständnisschwierigkeiten, aber ich glaube auch, dass die sozusagen weniger den Sinn gesehen haben oder so und dieses sehr instrumentelle Denken oder strategische Denken eher befremdlich fanden und ihnen auch fremd war, sagen wir mal so.

Juliane: $\quad$ Also haben die nicht mitgemacht, oder sind Dinge herausgekommen die nicht so $=$

Ökonomin: Ja, die haben ganz komisches Zeug gemacht, oder haben dann komische Fragen gestellt, wo Du dann denkst, Uh, weiß ich jetzt gar nicht was ich sagen soll'. Also, ich mein, das ist schon auch interessant, aber das kann man nachher natürlich nicht (.) mit denen müsste man halt reden, um irgendwas herauszubekommen. Was die dann nachher da machen, kannst Du ja in dem Sinne nicht irgendwie verstehen oder auswerten, das ist zu kompliziert. Also ja und die Universität X Studenten sind in der Hinsicht einfache Teilnehmer, die machen halt das. ((lacht)) “ (Auszug aus ExpertInneninterview1)

Hinsichtlich der hier betrachteten Frage der Rahmenbildung im Laboratop ist dies ein interessanter Verweis darauf, dass das Vorwissen und der wissenschaftliche Hintergrund der Teilnehmenden als einflussreich für die Produktion auswertbarer Ergebnisse betrachtet wird. Einen ,naturwissenschaftlichen oder mathematik- oder ingenieurwissenschaftlichen Background" $z$ u haben wird damit gleichgesetzt, dass man aus diesem Grund „gut mit Zahlen umgehen“ könne. Wer gut mit Zahlen umgehen kann, ist der Ansicht meiner Gesprächspartnerin folgend auch fähig, „halbwegs smart [...] mit Instruktionen umzugehen“. Ein „smarter Umgang“ mit den Instruktionen scheint einerseits darin zu bestehen, diese in einer Weise zu verstehen, wie sie von Seiten der Forschenden antizipiert wurde. Zugleich bedeutet dies jedoch auch, entsprechende instrumentelle 
oder strategische Handlungsstrategien abzuleiten und diese in den präsentierten Entscheidungssituationen des Experiments umzusetzen.

Die unterschiedlichen Voraussetzungen zwischen den VersuchsteilnehmerInnen an den verschiedenen Universitäten bedingte, den Aussagen der Gesprächspartnerin folgend, dass durch die Experimente Daten produziert wurden, die unterschiedlich gut für die Zwecke der Datenauswertung geeignet waren. Im Fall der TheologiestudentInnen waren die Ergebnisse scheinbar dadurch geprägt, dass sie die schriftlichen Instruktionen oder die von den Forschenden geplanten Handlungsabläufe nicht in der von den Forschenden antizipierten Weise, umsetzten konnten und aus diesem Grund Daten produzierten, die nicht ohne weiteres auszuwerten waren. Das von den Forschenden antizipierte Verständnis der Versuchsanordnung und der Regeln der Entscheidungsspiele stellte sich hier nicht wie erhofft ein. Strategisches und instrumentelles Denken scheint, der Ökonomin folgend, eine wichtige Vorbedingung zur angestrebten Bewältigung der Aufgaben und zugleich zur Produktion von Daten zu sein, die im Sinne der Forschenden auswertbar sind. Es wird damit nicht die Qualität der Entscheidungen der TheologiestudentInnen angezweifelt. Das Problem besteht vielmehr darin, dass die Transformation und Modulation, die sich im ökonomischen Laboratop vollzieht, auch an die ProbandInnen Ansprüche stellt, denen sie nicht alle ohne weiteres Folge leisten können (oder wollen).

Die im Experiment erhobenen quantitativen Daten sollen allein die Grundlage für die nachfolgenden Kausalanalysen bilden. Eine Ergänzung der quantitativen Daten durch weitere qualitative Daten ist im Verfahren des ökonomischen Laborexperiments nicht vorgesehen. Die soziale Rolle der ProbandInnen ist somit von Ansprüchen geprägt, die aus der Binnenlogik der Experimente hervorgehen.

\section{Laborteam}

Zusammengefasst besteht die Rolle der ProbandInnen grundlegend darin, in einem vorgegebenen Rahmen bestimmte Handlungen zu vollziehen und Handlungswahlen zu treffen, wobei sie sich darüber bewusst sind, dass sie dabei Daten für Forschungsvorhaben produzieren.

Die Aufgabe der ExperimentatorInnen besteht hingegen darin, optimale Bedingungen für einen reibungslosen Ablauf und eine valide Datenerhebung zu schaffen. Das Konstanthalten der Randbedingungen für die Teilnehmenden innerhalb und zwischen den einzelnen Experimenten ist dabei das zentrale Ziel. Der Begriff der ,Laborhygiene" scheint hier sehr passend. Es gilt das Labor im Allgemeinen und die spezifischen Experimente im Speziellen von potenziellen Störgrößen freizuhalten, um die ceteris paribus-Anforderung bestmöglich zu erfüllen und auf diese Weise zu begründen, warum die experimentellen Daten für den Nachweis 
eines Kausalzusammenhangs zwischen den getesteten Variablen tauglich sind. Die Aufgabe der ExperimentatorInnen besteht dabei einerseits darin, zu kontrollieren, dass sich die Teilnehmenden an die vorgegebenen Regeln halten. Zugleich wird eine größtmögliche Standardisierung der Laborsituation, des Ablaufs der Experimente und des allgemeinen Umgangs mit den ProbandInnen angestrebt. Zudem sind aus der psychologischen Experimentalforschung eine Reihe von Effekten bekannt, die die Datenerhebung beeinflussen und damit das Ergebnis verzerren können. Auch der Einfluss solcher Störgrößen soll vermieden werden. Die ExperimentatorInnen sind darum stets dazu angehalten, sich an den standardisierten Ablaufplan für die Datenerhebungen in den jeweiligen Treatments zu halten, um eine Verzerrungen der Ergebnisse zu verhindern. ${ }^{15}$

Es ist für die Forschenden wichtig, die Seriosität der Datenerhebung gegenüber den ProbandInnen sicherzustellen. Im Unterschied zur Psychologie gilt in der wirtschaftswissenschaftlichen Laborforschung ein Täuschungsverbot. ${ }^{16}$ Alle Informationen, die die ProbandInnen im Labor erhalten, sollen auch wahr sein. Den Teilnehmenden soll kein Grund dafür geben werden, einen Täuschungsversuch von Seiten der Forschenden zu vermuten. Die experimentellen Wirtschaftsforschenden gehen davon aus, dass nur so angenommen werden kann, dass die Versuchsbedingungen der Treatments in vergleichbarer Weise auf die ProbandInnen wirken. Zugleich stellt diese wissenschaftliche Redlichkeit aus ihrer Sicht eine Grundlage dafür dar, dass die angebotenen Informationen auch von den ProbandInnen ernst genommen werden. „Lügen“ ist in der ökonomischen Laborforschung somit nicht primär verpönt, weil dies ethischen Grundsätzen widerspricht, sondern um den potenziellen Einfluss von unkalkulierbaren Störvariablen - hier im Sinne von alternativen Situationsinterpretationen - besser zu kontrollieren.

In der von mir beobachteten empirischen Praxis wurde die im Labor agierende Gruppe der ExperimentatorInnen durch ein „Laborteam“ gebildet. Diese aus der Selbstbeschreibung der Handelnden stammende Terminologie umfasst dabei ein Team von geschulten studentischen MitarbeiterInnen, einer technischen Mitarbeiterin, die für die technische Betreuung und Programmierung zuständig war, und

\footnotetext{
${ }^{15}$ Bezuggenommen wird hier auf Versuchsleiter- bzw. Rosenthal-Effekte. Diese beziehen sich auf Verzerrungen der Ergebnisse eines Experiments, welche durch Motive, Umsetzungen oder Einstellungen einer VersuchsleiterIn beeinflusst werden. Ein Beispiel dafür wäre, wenn durch die Handlungen der ExperimentatorInnen signalisiert wird, welche Handlungen oder Reaktionen der Teilnehmenden wünschenswert wären (Demand-Effekt).

${ }^{16}$ Hier entspricht es durchaus der üblichen Praxis, dass die VersuchsteilnehmerInnen mittels einer „Cover Story“ über die eigentlichen Ziele der experimentellen Datenerhebung getäuscht werden.
} 
die jeweiligen Forschenden selbst, welche das Labor zur Datenerhebung nutzten. Die studentischen Mitarbeiter ${ }^{17}$ erfüllten dabei in der Regel die organisatorischen Aufgaben der Registrierung und Auszahlung der eingeladenen ProbandInnen. Zudem verfügten sie über das notwendige technische und praktische Wissen, die benötigten Programme im Labor zu starten und die Durchführung der Experimente im Labor zu betreuen. Sowohl die studentischen Mitarbeiter als auch die Forschenden werden im Rahmen der Arbeit als ExperimentatorInnen bezeichnet, wenn sie im Labor agieren.

\subsubsection{Die Orientierung an der wissenschaftlichen Peergroup}

Auch die Gründe dafür, dass einige Experimente im Rahmen des Brückenprojekts wieder verworfen wurden und sich die Projektgruppe anderen Themen zuwandte, stellen selbst wissens- und wissenschaftssoziologisch relevante Aspekte der Erkenntnisse über verhaltensökonomische Laborforschung dar. Sie zeigen auf, welche Kriterien erfüllt sein müssen, damit Forschungsideen im Labor weiterverfolgte werden (können). Zentral ist dabei beispielsweise, dass die Unterschiede zwischen verschiedenen Versuchsanordnungen zu signifikanten, messbaren Differenzen führen oder dass sich die Ergebnisse als bedeutsam für den aktuellen Diskurs der relevanten ökonomischen Peergroup herausstellen und somit zu publizierbaren Ergebnissen führen.

Dass sich die Orientierung an Standards und Ansprüchen der wissenschaftlichen Peergroup in der Durchführung und Konzeption der Experimente niederschlägt, zeigte sich beispielsweise in der Serie von Experimenten des „Exklusivitätsexperiments“" sehr deutlich. Es sollte hier untersucht werden, ob eine Wahloption attraktiver werden würde, wenn sie nur einem Teil der ProbandInnen als Auswahlmöglichkeit zur Verfügung stand, welche in einem vorher getätigten Spiel als GewinnerInnen hervorgegangen waren. ${ }^{18}$ Im Unterschied zu Eingangshypothese der WirtschaftswissenschaftlerInnen zeigte sich bei den GewinnerInnen

\footnotetext{
${ }^{17}$ Tatsächlich handelte es sich nur um männliche Mitarbeiter, weshalb hier nicht gegendert wurde.

${ }^{18}$ Um diese Entscheidungssituation im Labor abzubilden, wurde ein Experiment konzipiert, in dem die ProbandInnen zwischen unterschiedlich riskanten Lotterien wählen mussten. Riskant bedeutet hier, dass in den unterschiedlichen Lotterien verschieden hohe Wahrscheinlichkeiten vorlagen, zu verlieren, wobei zugleich alle Lotterien über vergleichbare Erwartungswerte verfügten und die potenziellen Gewinne somit bei zunehmenden Risiko deutlich anstiegen. Wie in der experimentellen ökonomischen Forschung üblich, wurde neben dem Versuchsaufbau, der zur Messung des interessierenden Effekts konzipiert wurde, ein Kontrolltreatment eingesetzt. In diesem Versuchsaufbau durften alle ProbandInnen zwischen drei Lotterien wählen. Die Ergebnisse wurden mit einem zweiten Treatment verglichen, in welchem nicht allen ProbandInnen alle drei Lotterien zur Auswahl standen. Um zu bestimmen, welche ProbandIn
} 
des Spieltreatments keine Verhaltensänderung. Die für sie ,exklusiv“ zugängliche Lotterie wurde von ihnen nicht signifikant häufiger gewählt als in der Kontrollgruppe. Überraschenderweise zeigte sich jedoch bei den VerliererInnen der Spiele eine signifikante Verhaltensänderung im Vergleich zur Kontrollgruppe. Diese ProbandInnen entschieden sich deutlich häufiger für die sichereren Varianten der Lotterie. Auch in den geführten Interviews nahmen einige der ProbandInnen direkten Bezug auf diesen Aspekt und berichteten, dass sie nach dem Erlebnis, im Spiel zu verlieren, nicht mehr auf ihr eigenes Glück setzen wollte. Aus diesem Grund entschieden sie sich für die Option, mit der geringstmöglichen Wahrscheinlichkeit beim Ausspielen der Lotterie zu verlieren. Trotz der signifikanten Ergebnisse, wurde diese Experimentserie nicht weiterverfolgt. Zentrales Argument unserer wirtschaftswissenschaftlichen ProjektpartnerInnen war, dass die Verhaltensänderungen von VerliererInnen kein ,hot topic“ in der Peergroup unserer experimentellen Wirtschaftsforschenden darstellt. Wie auch in einem anderen Gespräch mit den Forschenden expliziert wurde, orientieren sie sich bei der Wahl der Experimente deutlich an der Peergroup:

„Also viele Sachen sind ja eher so. Interessiert es nachher Leute. Ist es was, was Ökonomen finden das ist ein überzeugendes Argument oder nicht"

Eine Anschlussfähigkeit im aktuellen Diskurs und eine aussichtsreiche Chance auf eine Publikation in einem renommierten Fachjournal waren mit diesem unerwarteten Ergebnis nicht gegeben.

Der Erfolg der eigenen Forschungsleistung wird im Bereich der Ökonomie maßgeblich durch Veröffentlichungen in hochrangigen Fachzeitschriften ausgewiesen. Eine Orientierung und Einhaltung der Standards, die die Zeitschriften selbst setzen, ist dabei eine wichtige Voraussetzung für erfolgreiche Publikationen

aus allen drei Optionen wählen durfte, wurden die ProbandInnen in Zweiergruppen eingeteilt. Zu Beginn des Experiments wurden die Teilnehmenden durch schriftliche Instruktionen darüber informiert, dass sie im späteren Verlauf des Experiments die Chance erhalten würden, zwischen zwei Lotterien zu wählen. Zudem wurde ihnen mitgeteilt, dass sie durch den Gewinn in einem Spiel gegen einen anonymen, im Labor anwesenden Mitspielenden die Möglichkeit hätten, eine zusätzliche dritte Auswahloption zu erhalten. In dieser Konstellation spielten die Teilnehmenden nun ein sie ein kleines Glücksspiel. Es sollte dabei auf das Ergebnis eines Roulettewurfes getippt werden. SiegerIn des Spiels war diejenige, dessen Zahl (räumlich) dichter an der geworfenen Zahl war. Die GewinnerIn des Spiels durfte daraufhin zwischen drei Optionen wählen, wohingegen den VerliererInnen nur zwei mögliche Lotterien zu Auswahl standen. 
und zugleich für Karriere und Erfolgt innerhalb der (verhaltens-) ökonomischen Disziplin. Dies erweist sich beispielsweise hinsichtlich der Nutzung von monetären Incentives als relevant, welche einen vielfach geforderten Standard für die Veröffentlichung der Ergebnisse experimenteller Studien bilden.

Wie an anderer Stelle dargestellt, bilden Replikationen oder starke konzeptionelle Orientierungen an vorangegangenen Experimenten häufig den Ausgangspunkt für neue Experimente. Dies ist insbesondere im Anschluss an den methodologischen Anspruch des experimentellen Forschungsdesgins nachvollziehbar. Aufgrund der kontrollierten Bedingungen beansprucht das experimentelle Forschungsdesign des Laborexperiments - seinem Ideal folgend - sowohl eine hohe interne Validität der Ergebnisse als auch eine Wiederholbarkeit der Versuche, auf denen die Ergebnisse gründen. ${ }^{19}$ Im Sinne von Mertons Aphorismus bilden die „Schultern von Riesen“ häufig die Grundlage für eigene experimentelle Variationen und damit angestrebte eigene Beiträge zum Diskurs der Peergroup. Mit der Replikation einer Versuchsanordnung ${ }^{20}$ schließen Forschende somit an die Geltung des experimentell nachgewiesenen Kausalzusammenhangs und die Geltung dieser Ergebnisse an. Innerhalb einer Peergroup besteht der Forschungsprozess der experimentellen Wirtschaftswissenschaften maßgeblich in einem Prozess schrittweiser inkrementeller Weiterentwicklungen. Ergebnisse und Publikationen, die es erlauben, auf andere Autoren mit hohem Renommee Bezug zu nehmen und in der eigenen Forschung an diese anzuschließen, sind dabei besonders erstrebenswert. Auf diese Weise wird die Relevanz des eigenen Themas und der Ergebnisse in der Peergroup begründet und verstärkt. ${ }^{21}$

\subsubsection{Die Rekrutierung und Registrierung der ProbandInnen}

Zum Abschluss der Darstellung der Außenstruktur werden nun einige Aspekte des Laborexperiments expliziert, die zeitlich außerhalb der beobachteten Laborexperimente liegen. Auch wenn sich diese Arbeit auf die Analyse der Geschehnisse

\footnotetext{
${ }^{19}$ Dieses Nachstellen von legitimen und in der Peergroup der Forschenden anerkannten Ergebnissen bildet den Ausgangspunkt für die eigenen experimentellen Variationen. In diesen Fällen orientieren sich die Forschenden häufig auch an den Originalinstruktionen der Vergleichsstudie. Im Fall der Endowment-Effekt-USB-Stick Experimente diente beispielsweise eine wörtliche Übersetzung der englischen Originalinstruktionen als maßgebliche Grundlage für die eigenen durchgeführten Experimente im beobachteten Labor.

${ }^{20}$ Bei Veröffentlichungen von Ergebnissen experimenteller Studien ist es mittlerweile zudem üblich, die Instruktionen online zur Verfügung zu stellen.

${ }^{21}$ Ein Beispiel dafür liefert die Fn. 88 zur Untersuchung des Endowment-Effektes.
} 
im Labor konzentriert, ist es für die Betrachtung notwendig, dies nicht isoliert zu tun. Vielmehr soll das Laborgeschehen im Folgenden in den größeren sozialen Prozess eingeordnet werden, dem es entstammt. Die Prozesse des Anwerbens und der Registrierung der ProbandInnen sind maßgebliche Teile dieses Gesamtprozesses der experimentellen Forschung im Labor. Im Unterschied zur Ankunft der ProbandInnen vor dem Labor und zur Auszahlung der erspielten Gewinne sind sie jedoch keine Prä- und Postsequenzen des Ablaufs im Labor selbst. Das Anwerben und Registrieren sind zeitlich und (meist) auch räumlich vom Labor losgelöste Prozesse. Das typische Spannungsverhältnis von positiven und negativen Sanktionen und die Rollenerwartungen, die an die ProbandInnen im Labor gestellt werden, treten jedoch bereits hier deutlich zutage und bilden so Wissensbestände, die zugleich das spätere Geschehen im Labor prägen. In den geführten Interviews berichteten viele TeilnehmerInnen, dass sie bereits vor dem ersten Betreten des Labors spezifische Annahmen darüber hatten, was sie im Folgenden erwarten würde. Aus diesem Grund waren sie vom Verlauf der Ereignisse im Labor wenig überrascht. Im Folgenden werden deshalb kurz das Anwerben der ProbandInnen und ihre Registrierung im ProbandInnenpool dargestellt, da sie bereits hier Erwartungen und Vorkenntnisse sammeln, die als Wissensbasis in die Laborsituation einfließen und dort wirken.

Das Anwerben von zukünftigen ExperimentteilnehmerInnen erfolgt über verschiedene Wege. Eine Möglichkeit sind ausgehängte Plakate, an denen kleine Zettel abgerissen werden können. Zweitens stellen studentische MitarbeiterInnen des Labors die Experimente in Vorlesungen oder Übungen der Universität vor und werben für die Teilnahme. Eine dritte Möglichkeit besteht in der Mundpropaganda und somit in der Empfehlung von Freunden oder Bekannten.

Bereits beim Anwerben von TeilnehmerInnen für die beobachteten Laborexperimente wird explizit darauf verwiesen, dass diese Experimente eine einfache Möglichkeit sind, ,schnell Geld“ zu verdienen. Auf den Handzetteln und Postern, die zur Rekrutierung neuer ProbandInnen eingesetzt werden, springt dem Betrachter sofort der Slogan „Spielend Geld verdienen“ ins Auge. Auch die weiteren Erläuterungen bestärken die Fokussierung auf den monetären Anreizfaktor: Für die TeilnehmerInnen seien „keine Vorkenntnisse erforderlich“, um in einem Experiment einen „beachtlichen Betrag“ an Geld zu verdienen.

Der gesamte Text der Werbeplakate verweist, wie auf Abbildung 5.10 ersichtlich, zudem auf weitere bereits thematisierte Aspekte: „Spielend Geld verdienen! Das Fachgebiet für Experimentelle Wirtschaftsforschung führt regelmäßig Experimente durch, bei denen am Computer einfach Entscheidungen zu treffen sind. Dafür suchen wir Studierende aller Fachrichtungen. Es sind keine Vorkenntnisse 
erforderlich. Es kann ein beachtlicher Betrag, abhängig von den eigenen Entscheidungen und den Entscheidungen anderer Teilnehmer, verdient werden. Die Experimente dauern meist 1-2 Stunden. Wenn Sie an Experimenten teilnehmen wollen, tragen Sie sich bitte in folgende Datenbank ein, um Einladungen zu Experimenten zu erhalten“

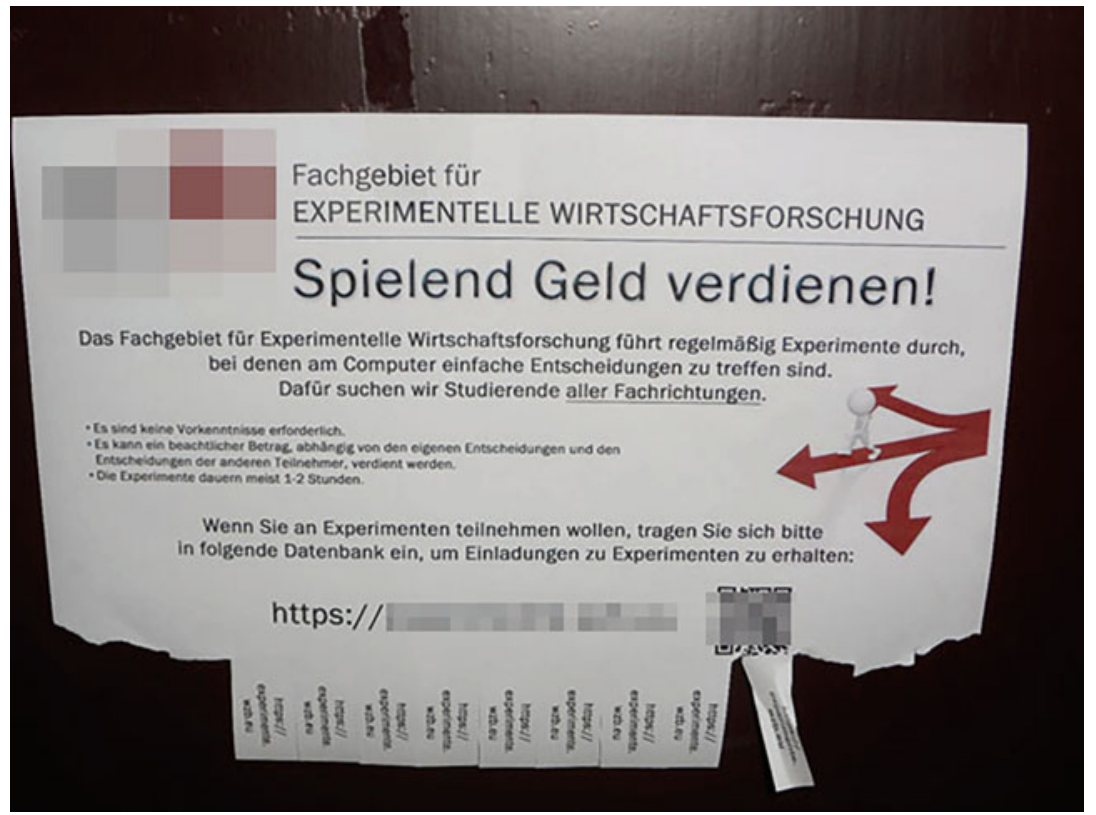

Abb.5.10 Werbeplakat

Alle interviewten ProbandInnen gaben den monetären Anreiz als primären Grund für ihre wiederholte Teilnahme an. Die leitende Verhaltenserwartung der Forschenden gegenüber den ProbandInnen, dass sie sich deutlich am in Aussicht gestellten Gewinn orientieren, ist somit nicht nur ein Teil des Akteurskonzepts der Forschenden. Diese Orientierung auf eine finanzielle Belohnung für die Experimentteilnahme wird von den ProbandInnen bereits im Vorfeld der Experimente als Entscheidungsprämisse für eine Teilnahme an den Experimenten übernommen und stimmt sie bereits auf ökonomische Rationalitätserwägungen ein. 


\section{Registrierung}

Entscheiden sich die Interessierten dafür, an diesen Experimenten teilzunehmen, so müssen sie sich via Internet in einer Online Datenbank anmelden. Die Registrierung erfolgt mit der speziell zu diesem Zweck konzipierten Software ORSEE ${ }^{22}$ (Greiner 2015). Dieses Tool dient der Depersonalisierung und Standardisierung der Interaktion zwischen ProbandInnen und ExperimentatorInnen. Die mittels der Datenbank registrierten StudentInnen melden sich nicht für ein spezifisches Experiment an, sondern erhalten von Seiten der ExperimentatorInnen Einladungen zu den jeweils stattfindenden Experimenten. Die Anmeldung bei ORSEE kann aber nur erfolgen, wenn die zukünftigen TeilnehmerInnen zustimmen, eine Reihe von Regeln des Experimentallabors zu befolgen:

- Nur wer seine persönliche Einladung zu einem Experiment per E-Mail erhält und diese mit einer positiven Rückantwort bestätigt, darf an einem Experiment teilnehmen.

- Die Bestätigung wird als verbindliche Zusage betrachtet und kann nur in begründeten Ausnahmefällen zurückgenommen werden.

- Zu spätes Absagen oder Nichterscheinen wird mit einem negativen Eintrag in das persönliche Konto der ProbandInnen sanktioniert. Diese Minuspunkte verringern die Chance, zu weiteren Experimenten eingeladen zu werden. Bei drei negativen Einträgen erfolgt keine weitere Einladung und der Account wird stillgelegt.

- Mehrfach im Rekrutierungssystem eingetragene Personen werden automatisch von den Experimenten ausgeschlossen.

- Die Interessierten werden darüber informiert, dass stets mehr TeilnehmerInnen eine Zusage für das Experiment erhalten, als tatsächlich benötigt werden. Am Experiment selbst nehmen diejenigen teil, die im Labor zuerst vor Ort sind und sich bei der MitarbeiterIn als Eingeladene ausweisen können.

- Wer rechtzeitig am Labor eintrifft, es jedoch nicht schafft, sich anzumelden, bevor alle freien Plätze im Labor vergeben sind, erhält eine Aufwandsentschädigung von 5 Euro. ${ }^{23}$

- Die TeilnehmerInnen stimmen darüber hinaus zu, sich an die in den Instruktionen festgelegten Regeln zu halten.

- Zudem werden die Teilnehmenden darüber informiert, dass sie üblicherweise in bar bezahlt werden.

\footnotetext{
${ }^{22}$ https://www.orsee.org/web/ (Zugriff am 28.11.2018)

${ }^{23}$ Diese Entschädigung wird auch als ,,Show-up fee“ bezeichnet.
} 
Diese Darstellung des Rekrutierungsprozesses zeigt, dass die zukünftigen ProbandInnen bereits vor ihrer Teilnahme an den eigentlichen Experimenten mit der Notwendigkeit und Strenge der Regelbefolgung und den Konsequenzen von Regelverletzungen konfrontiert werden. Nur durch die explizite Anerkennung der Regeln ist es möglich, sich für die Teilnahme an den Experimenten zu registrieren. Im Zuge ihrer Aufnahme in den Pool der ProbandInnen stimmen die Teilnehmenden dieser Art von Kontrolle zu. Regelbefolgung, Kontrolle, Standardisierung und die Fokussierung auf den monetären Anreizfaktor sind die zentralen Momente des Rekrutierungsprozesses und bilden eine Wissensbasis, die sich in der Durchführung der Experimente entäußert.

\subsubsection{Zusammenfassung der Ebene der Außenstruktur}

Wie in den vorangegangenen Ausführungen dargestellt, wird die Außenstruktur des ökonomischen Laboratops durch zentrale Wissenselemente und -hintergründe des sozialen Milieus gebildet, dem das ökonomische Laborexperiment entstammt. Um dies zu explizieren, wurde in diesem Abschnitt 5.2 auf forschungsleitende Konzepte der mikroökonomischen Experimentalforschung eingegangen. Einleitend wurde darauf verwiesen, dass die Standards der sozialwissenschaftlichen experimentellen Methodologie ebenfalls außenstrukturelle Aspekte des ökonomischen Laborexperiments bilden. Disziplinspezifische institutionelle Standards und Paradigmen bilden weitere außenstrukturelle Elemente. Es wurde in diesem Rahmen auf das Akteursmodell des Homo oeconomicus und Simons Konzept der ,begrenzten Rationalität‘ eingegangen. Aufgrund ihrer Relevanz für die Durchführung und Konzeption der Experimente wurden zudem forschungsleitende Paradigmen, wie die auf Kahneman und Tversky zurückgehende Idee des Framings in Entscheidungssituationen und die induzierte Werttheorie von Smith, dargestellt. Weitere Ausführungen widmeten sich den Charakteristika der sozialen Veranstaltung, wie der Verortung des sozialen Settings und den sozialen Rollen, die mit diesem Kommunikationsphänomen verbunden sind. Es wurde zudem auf die Orientierung an der wissenschaftlichen Peergroup für die Durchführung und Ausrichtung der Experimente verwiesen. Ein abschließender Abschnitt widmete sich dem Prozess des Anwerbens und der Registrierung von ProbandInnen und verwies darauf, dass diese bereits hier vielfältiges Orientierungswissen über die Situation des Laborexperiments gewinnen, welches sich in der Situation der konkreten Durchführung der Experimente als prägend und einflussreich erweist. 


\subsection{Merkmale der Binnenstruktur}

Die Perspektive der Gattungsanalyse lenkt den Blick auf die typischen und musterhaften Weisen des Lösens von Kommunikationsproblemen. Das Problem, welches es im wirtschaftswissenschaftlichen Experimentallabor zu lösen gilt, besteht darin, ein Entscheidungsumfeld für die ProbandInnen hervorzubringen, welches ihr ökonomisches Entscheidungsverhalten beforschbar macht. Eben diese Laborumwelt in ihrer spezifischen Materialität und Sozialität wird von mir als ökonomisches Laboratop bezeichnet. Die auf der Ebene der Außenstruktur diskutierten Elemente bilden als institutionalisierte Wissensbestände und Strukturmerkmale den Kontext des Phänomens des ökonomischen Laboratops. Während die Darstellung der Außenstruktur institutionelle Aspekte und paradigmatische Annahmen fokussierte, welche das Phänomen des Laborexperiments prägen, geht es im Folgenden darum, zu betrachten, wie diese Aspekte in empirisch beobachtbarer typischer Art und Weise umgesetzt werden. Die einzelnen außenstrukturellen Elemente sind auf dem Weg dieser Zielerreichung mit unterschiedlichen Formen kommunikativen Handelns verbunden. Der durch die Forschenden induzierte Prozess der Rahmenbildung ist dabei darauf ausgerichtet, die Situationsdefinition der ProbandInnen und somit ihre Rationalitäten denen der Beforschten bestmöglich anzunähern. Im Sinne Goffmans können die nachfolgend thematisierten musterhaften Formen kommunikativen Handelns somit als Verankerungsversuche eines gezielt induzierten Rahmens verstanden werden. Sie weisen damit aus, welche Aspekte aus Sicht der Forschenden für den Prozess der Rahmenbildung als relevant erachtet werden und zugleich wie sie anstreben, diese als die naturwüchsige typische Ordnung des wirtschaftswissenschaftlichen Experimentierens zu (re-)produzieren. Während auf der Analyseebene der Außenstruktur die Randomisierung beispielsweise ein wichtiges Element des experimentellen Forschungsdesigns bildet, wird auf Ebene der Binnenstruktur betrachtet, wie dieser Anspruch in der Konzeption und Durchführung von Experimenten auf standardisierte Weise umgesetzt wird und welchen Beitrag diese Umsetzung in der Konstruktion des ökonomischen Laboratops leistet.

Die Rahmenbildung wird dabei als ein interaktiver Prozess verstanden, welcher sich durch die accounting practices der Handelnden vollzieht. Die Produktion und Reproduktion des sozialen Phänomens ist dabei ein stets gleichzeitig ablaufender Prozess. Das Charakteristische des ökonomischen Laborexperiments, seine spezifische Art der Labor- und Experimenthaftigkeit konstituiert sich somit im beobachtbaren sozialen Prozess des Experimentierens selbst. Sowohl auf Seiten der ExperimentatorInnen als auch auf Seiten der ProbandInnen materialisiert 
sich das Verstehen des Rahmens und eine Orientierung an diesem somit maßgeblich im beobachtbaren Handeln. Die Merkmale der Ebene der Binnenstruktur und der situativen Realisierung werden durch Ethnomethoden der Handelnden und charakteristische Objektivationen gebildet. Die binnenstrukturelle Analyseebene fokussiert dabei solche Merkmale und Muster der Konstruktion des Entscheidungsumfeldes im Labor, die situationsunabhängig sind, wohingegen sich die Analyseebene der situativen Realisierung den Aspekten widmet, die zur interaktiven Handlungskoordination beitragen.

Im Vollzug der Analyse wurde eine Analyseheuristik gebildet, um den spezifischen Beitrag der rahmungsrelevanten musterhaften Formen kommunikativen Handels differenziert zu betrachten. Diese zielt darauf, auszuweisen, welchen ,funktionalen' Beitrag die einzelnen Merkmale an der prozesshaften Verankerung und Hervorbringung des Rahmens besitzen. Um die Prozesshaftigkeit der Rahmenbildung zu unterstreichen, wird dieser als das „Doing“ des ökonomischen Laboratops bezeichnet. Es werden in der Darstellung der Analyseergebnisse vier Ebenen des Doings unterschieden. Die Analyseheuristik erlaubt es dabei, zu betrachten, welche spezifische Form der Modulation des primären Rahmens des Alltags hier durch den Prozess der Rahmenbildung angestrebt und hervorgebracht wird und wie die unterschiedlichen induzierten Rahmungsebenen ein phänomenspezifisches Mischungsverhältnis von Moduln (im Sinne Goffmans) ausbilden.

Auf der ersten allgemeinsten Ebene kann man vom Doing einer jeden sozialen Praxis sprechen, in der sich die Akteure in ihren Handlungen wechselseitig situativ aneinander orientieren, um so eine gemeinsame Situationsdeutung und eine geteilte soziale Wirklichkeit zu produzieren. Bei diesen Aspekten liegt keine spezifische Transformation oder Modulation einer primären Rahmung vor. Mit dem Ziel der Fokussierung auf die Verankerungsmethoden im Prozess der Rahmenbildung des ökonomischen Laboratops wird davon die zweite Ebene des Doings einer spezifischen Laborsituation unterschieden. Die mit dieser Ebene verbundenen Praktiken erlauben es, eine Situation zu schaffen, die den zentralen Prämissen der Forschung im Labor entspricht und somit beispielsweise die Reproduzierbarkeit der Ergebnisse oder die Kontrolle von Störvariablen ermöglicht. Die hier thematisierten Aspekte sind hinsichtlich ihrer Rahmungsleistung als Ausdruck des Experimentmoduls zu verstehen.

Von dieser zweiten Ebene sind zwei weitere Ebenen des Doings zu unterscheiden, die spezifische Besonderheiten des verhaltensökonomischen Settings ausbilden. Auf einer dritten Ebene lässt sich das Doing der rationalen und regelkonformen Verhaltenserwartungen bei den ProbandInnen unterscheiden. Bereits mit Beginn des Rekrutierungsprozesses für die Experimente werden vielfältige 
Techniken eingesetzt, um den TeilnehmerInnen aufzuzeigen, dass regelkonformes Verhalten von ihnen erwartet und eine Zuwiderhandlung von den ForscherInnen sanktioniert wird. Die Art der Transformation einer primären Rahmung, welche durch die Handlungen erzielt wird, kann als eine spezifische Art des Spielmoduls gefasst werden. Bei diesem sind insbesondere die Regeln des Spiels und die gezielte Konstruktion einer Spielsituation zentral.

Auf einer vierten Ebene findet sich schließlich das Doing der spezifisch ökonomisch-rationalen Verhaltenserwartungen. Hier stehen die Methoden und Praktiken im Fokus, die die Forschenden einsetzen, um den ProbandInnen eine ökonomische Situationsdefinition nahezulegen, wie beispielsweise einen monetären Anreiz für die Teilnahme am Experiment oder für das Verhalten in den Experimenten. Ziel dieser Methoden ist es, die Übernahme der Sprach- und Verhaltensspiele der Forschenden durch die ProbandInnen zu begünstigen. Die ProbandInnen wiederum orientieren sich bereitwillig an diesen Verhaltenserwartungen oder weichen aufgrund differierender Situationsdeutungen von diesen ab. Hier zeigt sich das Modul der Mikroökonomie und der ökonomisch relevanten Entscheidungssituationen.

Für die nachfolgend diskutierten Aspekte auf der Analyseebene der Binnenstruktur stellen die interaktions- und situationsunabhängige Dauerhaftigkeit der kommunikativen Muster das zentrale Auswahlkriterium dar. Der spezifische Fall des betrachteten empirischen Phänomens bedingt dabei, dass die räumlichmaterielle Spezifik des Laborraums hier ebenfalls als Merkmal auf der Ebene der Binnenstruktur gefasst wird. Argumentativ wird hier Bezug auf den erweiterten Begriff kommunikativen Handelns im Sinne des kommunikativen Konstruktivismus genommen. Dieser erlaubt es auch, die in Objekten eingeschriebenen Wissensbestände, die sich in empirischen Situationen als wirkmächtig erweisen, als Objektivationen zu erfassen. Es werden hier somit auf der Analyseebene der Binnenstruktur Aspekte aufgenommen, die der ,klassischen Zuordnung ' nach Luckmann $^{24}$ (1992) zuwiderlaufen.

\footnotetext{
${ }^{24}$ Laut Luckmann (1989) lassen sich die Merkmale der Binnenstruktur wie folgt charakterisieren: „Die Binnenstruktur einer Gattung besteht aus: Gesamtmustern recht unterschiedlicher Elemente: aus Worten und Phrasen, Gesamtregistern, Formeln und formularischen Blöcken, rhetorischen Figuren und Tropen, Stilmitteln wie Metrik, Reimschemata, Listen, Oppositionen usw., Lautmelodien, spezifischen Regelungen der Dialogizität wie Redezugbestimmungen, Reparaturstrategien und Festlegungen von Themen und Themenbereichen“" (ebd.: 39).
} 


\subsubsection{Gliederungsmerkmale des sozialen Prozesses des Experiments}

Ein charakteristisches Moment des Handlungsprozesses im wirtschaftswissenschaftlichen Labor ist das Bestreben der ExperimentatorInnen, die Situation im Labor so weit wie möglich zu kontrollieren. In der empirischen Umsetzung dieses Anspruchs erfolgt dies maßgeblich durch den Versuch, vielfältige Elemente und Handlungsschritte zu standardisieren. Dies gilt auch für die Gliederung des Prozesses der durchgeführten Experimente im Labor. Es lässt sich eine charakteristische Abfolge deutlich voneinander getrennter Handlungssequenzen ausmachen. Als einleitender Aspekt der Analyseebene der Binnenstruktur werden nun die Gliederungsmerkmale des Handlungsprozesses dargestellt. Die nachfolgende Liste der Gliederungsmerkmale ist das Ergebnis einer Synthese aus teilnehmenden Beobachtungen und beobachtender Teilnahme bei der Durchführung von Experimenten.

\section{Betreten des Labors durch die Teilnehmenden und Platzsuche}

Alle ProbandInnen treten ein und setzen sich an den Platz, der der Nummer ihres Spielchips entspricht. Die am Experiment beteiligten Mitglieder des Laborteams betreten ebenfalls den Laborraum und versammeln sich an einem separaten Tisch am Kopfende des Labors.

2. Begrüßung der Teilnehmenden und Vorstellen der Verhaltensregeln

Der Übergang zum nächsten Handlungsabschnitt wird mit dem Redebeginn eines Mitglieds des Laborteams markiert, welches die ProbandInnen im Labor willkommen heißt. Der Inhalt dieser Begrüßung ist standardisiert. Die ProbandInnen werden über die zentralen Regeln für ihr Verhalten im Labor informiert. Es ist den Teilnehmenden erstens untersagt, mit anderen Teilnehmenden zu kommunizieren. Es ist ihnen zweitens nicht erlaubt, andere technische Geräte oder Programme des Computers zu nutzen. Als dritte Regel gilt, dass Fragen von den Teilnehmenden nicht laut gestellt werden dürfen, sondern durch Handzeichen angezeigt werden müssen. Im Zuge dieser Belehrung werden sie auch auf die potenziellen Konsequenzen bei Zuwiderhandlung aufmerksam gemacht.

3. Lesen der Instruktionen

Auf dem Arbeitsplatz jedes Teilnehmenden liegen mehrere bedruckte Seiten mit schriftlichen Instruktionen aus. Am Ende der Begrüßung werden die Teilnehmenden aufgefordert, mit dem Lesen der Instruktionen zu beginnen. Je 
nach Länge der Instruktionen wird nach einem angemessenen Zeitraum erfragt, ob alle Teilnehmenden das Lesen der Instruktionen abgeschlossen haben. Mittels Handzeichen signalisieren die Teilnehmenden, wenn sie noch mit dem Lesen beschäftigt sind. Diese Nachfrage wird gegebenenfalls mehrfach wiederholt, bis keiner der anwesenden Teilnehmenden mehr aufzeigt.

4. Zeit für die Beantwortung von Nachfragen der Teilnehmenden

Während die ProbandInnen die Instruktionen lesen, achten die anwesenden Mitglieder des Laborteams darauf, ob einer der Teilnehmenden ein Handzeichen gibt und so signalisiert, dass er bzw. sie eine Frage hat. Sieht einer der ExperimentatorInnen eine erhobene Hand, so begibt er sich umgehend dorthin und beantwortet die Frage in einem persönlichen und leisen Zwiegespräch. Nachdem alle ProbandInnen signalisiert haben, dass sie das Lesen der Instruktionen beendet haben, wird noch einmal erfragt, ob es noch Nachfragen zu den Instruktionen gibt. Ist dies nicht der Fall wird zur nächsten Handlungssequenz übergegangen.

5. Beginn des spezifischen Experiments am Computer

Nach den einleitenden Handlungssequenzen beginnt der für die Datenerhebung relevante Teil des Handlungsprozesses. Die Teilnehmenden sollen nun den schriftlichen Instruktionen und zusätzlichen verbalen Anweisungen der Forschenden Folge leisten. Die Inhalte der einzelnen Experimente sind von den spezifischen Fragestellungen abhängig. Teile des Experiments beinhalten jedoch stets die Bearbeitung von Aufgaben am Computerbildschirm.

6. Fragebogen

Im Anschluss an den auszahlungsrelevanten Teil des Experiments werden die Teilnehmenden gebeten, am Computer einen Fragebogen auszufüllen. Ihnen wird mitgeteilt, dass ihre Antworten keinen Einfluss auf ihren erspielten Gewinn haben. Die Minimalversion des Standardfragebogens erfasst grundlegende demografische Informationen (Alter, Studienfach, Semesteranzahl). Zudem wird den ProbandInnen in einem Freifeld die Möglichkeit geboten, Lob und Kritik zum Experiment, an dem sie gerade teilgenommen haben, zu vermerken.

7. Ausfüllen und Kontrolle der Quittungen

Während die Teilnehmenden den Fragebogen ausfüllen, verteilen die ExperimentatorInnen bedruckte A5 Blätter, die als Quittungen fungieren. Nach dem Ausfüllen des Fragebogens tragen die ProbandInnen ihren im Experiment erzielten Geldbetrag, ihren Namen und ihre Matrikelnummer in den Quittungsvordruck ein. Die ExperimentatorInnen ziehen im Labor ihre Kreise, 
überprüfen die Richtigkeit des eingetragenen Geldbetrags und vermerken mittels eines kleinen Zeichens auf der Quittung, dass sie diese kontrolliert haben.

8. Verlassen des Laborraums

Nach der Kontrolle der Quittungen ist es den Teilnehmenden erlaubt, den Laborraum zu verlassen, um ihre Auszahlung abzuholen.

In den Interviews wurden die teilnehmenden ProbandInnen gebeten, den typischen Ablauf der Laborexperimente zu schildern, um Auskunft über die von ihnen wahrgenommenen und antizipierten Schritte in dem Handlungsprozess zu erhalten. Die zwei nachfolgenden Interviewausschnitte ${ }^{25}$ stellen typische Darstellungen des Handlungsablaufs aus Sicht der ProbandInnen dar. Der erste Ausschnitt entstammt einem Interview mit den beiden Probanden Roland und Dennis.

Interviewerin 1: Könnt Ihr mal bitte die Schritte beschreiben, die so typisch sind für so ein Laborexperiment? (...)

Dennis: $\quad$ Also ich versuche immer, 10 Minuten vorher da zu sein. Dann wartet man eben die Zeit ab, bis die Nummern verteilt werden und dass eben die Anwesenheit kontrolliert wird. Dann kommt man ins Experiment rein und setzt sich aufden Platz, dann liegen meistens die Instruktionen schon aus, die fange ich dann an zu lesen. Ja, dann gibt es den Hinweis, dass sie gelesen werden sollen, dann bin ich meistens schon bei der Hälfte, dann lese ich sie zu Ende.

Roland: $\quad$ Dann werden sie noch mal vorgelesen.

Dennis: $\quad$ Dann werden sie noch mal laut vorgelesen, genau.

Interviewerin1: Nachdem man sie gelesen hat, werden die noch mal gelesen?

Roland: $\quad$ Genau.

Dennis: $\quad$ Obwohl nicht immer.

Roland: $\quad$ Ja stimmt nicht immer. Manchmal gibt's dann auch so ein Verständnistest.

${ }^{25}$ Im Sinne der besseren Lesbarkeit des Interviewausschnitts wurde orthographisch richtig transkribiert. 
Dennis: $\quad$ Ja manchmal, stimmt, den hatte ich aber schon seit drei-, viermal nicht mehr, aber ansonsten noch ein Verständnistest. $[\ldots]^{26}$

Roland: $\quad$ Und dann startet das Experiment, meistens sind dann ja die Instruktionen auf dem Bildschirm und ja, dann kommt es eben ab und zu mal zu Wartezeiten, eben weil man noch auf die anderen Experimentteilnehmer warten muss. Ja, dann ist es irgendwann zu Ende. Dann geht man da rüber, Geld holen.

Dennis: $\quad$ Genau, man lässt sich den Zettel unterschreiben, dann geht man rüber, holt sich das Geld ab und dann geht man nach Hause.

Auch in einem zweiten Interviewausschnitt verweist die Darstellung der Probandin Lydia auf die typische Gleichartigkeit des Handlungsablaufs:

Lydia: $\quad$ Eigentlich läuft das immer gleich ab, habe ich das Gefühl. Man wartet vor der Tür, dann kommt jemand raus. Man zieht dieses Ding und setzt sich hin. Man hat meistens relativ viel Zeit zum Lesen und dann wird's noch mal durchgegangen oder noch mal vorgelesen oder noch mal erklärt. Also alles auch sehr, sehr slowly, also so, dass wirklich jeder mitkommt, gefühlt. Und auch die Zettel sind ja sehr ausführlich und oft wiederholt und dann läuft das Experiment ab und dann mit der Quittung. Also eigentlich (.) gefühlt läuft das immer gleich ab. Es gibt natürlich immer so kleine Besonderheiten wie jetzt hier, wo dann dieser USB gereicht wird oder so, aber an sich, gefühlt, laufen die wirklich immer gleich ab.

Wie ersichtlich wird, führen die Interviewten hier nicht alle Aspekte an, welche oben als Gliederungsmerkmale geschildert wurden. So werden die anfängliche Belehrung und die Vorstellung der Regeln von ihnen ebenso wenig erwähnt wie das Stellen von Nachfragen oder das Ausfüllen des Fragebogens. Die beiden Interviewausschnitte zeigen hingegen auf, dass eine Ordnung des Ablaufs

\footnotetext{
${ }^{26}$ An der Stelle der Auslassung wurde über das Vorgehen des Einsatzes von Verständnistest in den Experimenten gesprochen. Da dies für den dargestellten Argumentationszusammenhang nebensächlich ist, wurde dieser Teil zur, Verschlankung' des Ausschnitts ausgespart.
} 
nicht nur aus der Beobachterperspektive, sondern auch aus Sicht der Teilnehmenden zu erkennen ist. Mehrfach weist die Probandin Lydia darauf hin, dass die Experimente ,gefühlt“ immer gleich ablaufen. Alle dazu befragten ProbandInnen konnten in den Interviews auf Nachfrage typische Merkmale des Handlungsprozesses benennen. Hinsichtlich der Hervorbringung des untersuchten Phänomens des ökonomischen Laboratops ist dies von Belang: Die Kenntnis der Phasen des Handlungsprozesses und die Orientierung an diesen sind wichtige Wissenselemente der Ethnomethoden der Handelnden. Gerade nach mehrmaliger Teilnahme an Experimenten sind ihnen die typischen Handlungsschritte und der allgemeine Ablauf sehr vertraut. Eine deutliche Varianz nehmen die ProbandInnen nur hinsichtlich der spezifischen Inhalte der Experimente wahr.

Wie im Analyseteil dargelegt, wird als außenstruktureller Aspekte von Seiten der Labormitglieder die Kontrolle über das Setting der Datenerhebung angestrebt und damit zugleich ein Konstanthalten der Randbedingungen. In diesem Sinne sind gleichbleibende und standardisierte Handlungsabläufe $\mathrm{zu}$ bevorzugen, da auf dieses Weise ausgeschlossen werden kann, dass Veränderungen des Ablaufs Irritationen bei den ProbandInnen hervorrufen. und sich auf die Datenerhebung als Störvariable auswirken (könnten). Der standardisierte Ablauf des allgemeinen Handlungsprozesses trägt somit zur Kontrolle im Laboratop bei und dient hier als ein Schritt der praktischen Umsetzung des Konstanthaltens der Randbedingungen. Das Konstanthalten der Randbedingungen jedoch nicht nur in einer Serie von Experimenten angestrebt, sondern auch über verschiedenen Serien von Experimenten hinweg, die in dem gleichen Labor durchgeführt werden. Dieses Vorgehen der Standardisierung von Abläufen trägt somit gleichsam zur Reliabilität der im Labor erhobenen Daten bei. Die Orientierung an gleichbleibenden Gliederungsmerkmalen bildet in diesem Sinne einen Beitrag zum Doings einer spezifischen Laborsituation und ist Teil des Experimentmoduls.

\section{Prä- und Postsequenzen}

Wie in den exemplarischen Ausführungen der Interviewten zu den Gliederungsmerkmalen des Laborexperiments dargestellten, wird nicht nur das Handlungsgeschehen im Labor als typischer Bestandteil des Phänomens betrachtet. Für die Interviewten sind auch die Vorbereitungen vor dem Labor, der Einlass der ProbandInnen sowie die Auszahlung nach dem Experiment mit dem Geschehen im Labor eng verbunden. Das Phänomen wird somit von typischen Prä- und 
Postsequenzen eingerahmt. ${ }^{27}$ Bezüglich der interaktiven Handlungskoordination erfüllen die Sequenzen wichtige Voraussetzungen für den geordneten Ablauf des Handlungsprozesses im Labor. ${ }^{28}$ Aus diesem Grund werden diese Handlungssequenzen im Folgenden beschrieben. Zudem wird auf die Ebenen des Doings der Situationsrahmung verwiesen, zu welchen diese einen Beitrag leisten.

\section{Die Ankunft der ProbandInnen und die Vorbereitungen vor dem Labor}

Bevor das Experiment im Labor beginnt, finden sich die eingeladenen Personen im Flur vor dem Laborraum ein. Einige Minuten vor dem angegebenen Beginn des Experiments erscheint ein Mitglied des Laborteams mit einer Liste in der Hand und begrüßt die Anwesenden freundlich. Sie werden von ihm gebeten, nun bitte einzeln zu ihm zu kommen, um mittels eines Ausweisdokuments und durch einen Datenabgleich sicherzustellen, dass es sich bei ihnen auch tatsächlich um die eingeladenen ProbandInnen handelt. Wenn die Wartenden an das Labormitglied herantreten, hat der Großteil von ihnen bereits ein Ausweisdokument in der Hand, um es ihm vorzulegen. Der Mitarbeitende wiederum hält eine Liste in der Hand, auf der die ProbandInnen ihre Teilnahme quittieren. Anschließend ziehen die Teilnehmenden einen kleinen nummerierten Spielchip aus einem schwarzen Stoffbeutel. Das soziale Setting wirkt bereits vor der Ankunft des Labormitarbeiters recht kühl. Dadurch, dass alle Wartenden auf den Mitarbeitenden zugehen, wird ersichtlich, dass sie das Erscheinen dieser Person als Auftakt für das beginnende Experiment identifizieren. Nur wer der Aufforderung des Labormitglieds nachkommt und sich einzeln und diszipliniert als eingeladene ProbandIn ausweist, kann an dem nachfolgenden Experiment teilnehmen. Dadurch, dass die Wartenden der Bitte des Mitarbeitenden Folge leisten, erkennen sie zugleich seine Rolle des Labormitgliedes an und sein Recht, sie zu derartigem Handeln aufzufordern.

Hinsichtlich der im Zuge der Außenstruktur beschriebenen Aspekte werden durch diese Vorbereitungen wichtige Grundlagen für die Schaffung der gewünschten Situation im Labor gelegt. Wie in den Ausführungen zur Methodologie sozialwissenschaftlicher Experimente (Abschnitt 2.1) ausgeführt, besteht

\footnotetext{
${ }^{27}$ Die im Rahmen dieser Arbeit vorgenommene Fokussierung, auf das interaktive Geschehen im Labor, bedingt, dass diese Handlungssequenzen, trotz ihres Stellenwerts für die Hervorbringung des Phänomens, nicht als integriertes Gliederungsmerkmal gefasst werden.

${ }^{28}$ Analog dazu verweist Bergmann (1987) in seiner Analyse von Klatschsituationen darauf, dass in der Präsequenz einer Klatschkommunikation erst einmal geklärt werden muss, ob die Voraussetzungen für die Aufnahme einer solchen Interaktion gegeben sind.
} 
das zentrale Anliegen bei Experimenten in der Kontrolle und Isolation der hypothesenrelevanten Variablen. Das Verfahren der Randomisierung ${ }^{29}$ stellt dabei ein zentrales Definitionskriterium des experimentellen Designs von Laborexperimenten dar. Im Rahmen der Laborexperimente erfolgt die Randomisierung maßgeblich im Zuge der Einladung und Anmeldung der ProbandInnen zu den einzelnen Experimenten. Basierend auf bestimmten Ausschlusskriterien wird dabei die Teilmenge des ProbandInnenpools bestimmt, welche die für das Experiment nötigen Voraussetzungen erfüllt. ${ }^{30}$ Es werden stets mehr ProbandInnen angeschrieben, als für das Experiment benötigt werden. Welche der angeschriebenen ProbandInnen sich zurückmelden und sich für die offenen Plätze in der Session anmelden, liegt außerhalb des Einflussbereichs der Mitglieder des Laborteams und wird von ihnen deshalb als zufällig betrachtet. Die Anmeldung der ProbandInnen für die einzelnen Sessions der Experimente erfolgt dabei ohne Kenntnis über den spezifischen Inhalt oder darüber, welche anderen ProbandInnen noch an diesem Experiment teilnehmen werden. Die Kontrolle der Ausweisdokumente der vor dem Labor befindlichen Personen und ihr Abgleich mit der Liste der Eingeladenen ermöglicht es dabei zu kontrollieren, dass die gewünschte Zusammenstellung der Stichprobe praktisch auch realisiert wird. Dieses Vorgehen trägt somit zum Doing einer standardgerechten Experimentsituation bei und bildet einen Aspekt des Experimentmoduls der Rahmenbildung. Das Verfahren der Randomisierung auf der Ebene der Auswahl und Einladung der ProbandInnen bildet aus Sicht der Forschenden ein zentrales Kriterium des Experimentmoduls der Rahmung bei der Vorbereitung der Laborexperimente. Es ist ein wichtiges außenstrukturelles Definitionskriterium ebendieser.

Doch auch im Labor selbst erfolgt eine randomisierte zufällige Verteilung auf die zur Verfügung stehenden Arbeitsplätze, um einen systematischen Einfluss

\footnotetext{
${ }^{29}$ Randomisierung bezeichnet die zufällige Zuweisung von ProbandInnen zu Kontroll- und Versuchsgruppen. Mit diesem Vorgehen wird der Anspruch verbunden, den unkontrollierten Einfluss bekannter und unbekannter Störvariablen statistisch auszuschalten oder zumindest so weit zu minimieren, dass sie die Ergebnisse der Datenerhebung nicht maßgeblich verzerren (siehe auch Abschnitt 2.1).

${ }^{30}$ ProbandInnen werden bei ihrer Anmeldung gebeten, gewisse Informationen über sich preiszugeben, wie Alter, Geschlecht und Studienfach. Zudem wird intern im Sinne einer „ProbandInnenhistorie“ vermerkt, an welchen Experimenten welche ProbandInnen teilgenommen haben. Diese Informationen bilden die Grundlage für die Selektion der passenden Teilgruppe des ProbandInnenpools, welche zur Teilnahme an den Experimenten eingeladen wird. Geschlecht (im Sinne einer angestrebten Gleichverteilung), Anzahl der Experimentteilnahme oder Vorerfahrungen mit spezifischen Typen von Experimenten bilden dabei meist relevante Selektionskriterien.
} 
potenziell unerwünschter Einflussfaktoren zu minimieren. In der Vorbereitungsphase erfolgt der erste Schritt zur Umsetzung der Randomisierung im Labor durch den Einsatz der Spielchips, welche die Teilnehmenden blind aus einem kleinen Stoffbeutel ziehen. Der nachfolgende Abschnitt 5.3.2 wird sich diesem Aspekt im Detail widmen.

Wie im Abschnitt 5.2.4 auf der Ebene der außenstrukturellen Aspekte dargestellt, vermitteln die Prozesse des Anwerbens, der Registrierung und Einladung der ProbandInnen relevante Informationen über den Ablauf und die Durchführung der Experimente selbst. Zugleich weisen sie selbst eine Orientierung an außenstrukturellen Aspekten auf. Diese Vorphasen sind, bezogen auf die Kommunikation mit den ProbandInnen, stark standardisiert und verwenden gezielt bestimmte Software-Programme (wie ORSEE), um eben diese Gleichartigkeit zu erzielen. Auf diese Weise tragen sie zum Doing einer standardgerechten Experimentsituation und zur Verankerung des Experimentmoduls des ökonomischen Laboratops bei. Darüber hinaus trägt der Prozess der Ankunft und Registrierung zugleich aktiv zum Doing regelkonformer und rationaler Verhaltenserwartungen bei. Die Befolgung der Regeln, die im Zuge der Registrierung und Einladung vermittelt wurden, ist eine maßgebliche Voraussetzung für die Teilnahme an den Experimenten. Es handelt sich hier um erste Schritte der Verankerung des spezifischen Spielmoduls, welches für das ökonomische Laboratop charakteristisch ist. Es werden erste Regeln der ,Spielwelt “ ausgewiesen, die im ökonomischen Laboratop Geltung besitzen. Bereits hier wird das experimentspezifische Belohnungsund Bestrafungssystem eingesetzt, welches die monetäre Motivation nutzt, um gewünschte und geforderte Verhaltensweisen bei den ProbandInnen zu befördern. Dies erfolgt einerseits durch die Sanktionsandrohung des negativen Eintrags in die persönliche Akte der ProbandInnen. Andererseits wird für das pünktliche Erscheinen ein Gewinn in Aussicht gestellt, der im Experiment erzielt werden kann oder in der Minimalversion die Show-up fee von 5 Euro umfasst. Im Sinne des Spielmoduls wird die Transformation oder Modulation in eine soziale Situation vorbereitet, welche von den ProbandInnen eine Orientierung an einem vorgegebenen Regelrahmen erwartet. Auch das Sanktionssystem für regelkonformes Verhalten wird dabei bereits in dieser Vorphase eingeführt.

\section{Die Auszahlung der ProbandInnen}

Wie gerade beschrieben, erfüllen die Vorbereitungen und Registrierungen vor dem Labor als Präsequenz entscheidende Vorbedingungen für den typischen Ablauf des Interaktionsgeschehens und die Rahmenbildung des ökonomischen Laboratops selbst. Auch nach dem Verlassen des Labors schließt sich eine typische 
Postsequenz an, welche entscheidende Bedeutung für die Rahmung des Laboratops besitzt. Es handelt sich bei dieser Postsequenz um die Auszahlung der ProbandInnen im Nebenraum. Wie in den Gliederungsmerkmalen (s. o.) ausgeführt, wird den Teilnehmenden typischerweise kurz vor Ende (des im Labor durchgeführten Teils des Experiments) am Computerbildschirm angezeigt, wie viel Geld sie sich erarbeitet haben. Diesen Betrag tragen die Teilnehmenden in eine Quittung ein und lassen diese von einem Mitglied des Laborteams gegenzeichnen. Im Anschluss verlassen die Teilnehmenden den Laborraum. Die Auszahlung erfolgt außerhalb des Laborraums in einem Nebenzimmer. Einzeln treten die Teilnehmenden hier an eine dort befindliche ExperimentatorIn heran und erhalten gegen Vorlage der Quittung den eingetragenen Geldbetrag. ${ }^{31}$ Die ProbandInnen erhalten typischerweise Bargeld. ${ }^{32}$ Die Quittungen verbleiben zum Zwecke der Abrechnung bei dem Mitglied des Laborteams. Anschließend verlassen die ProbandInnen den Raum - das Experiment ist für die Teilnehmenden nun offiziell beendet.

Auch wenn der Pool der ProbandInnen des untersuchten Experimentallabors mit 4000 Personen recht groß ist, hat die Mehrzahl der ProbandInnen bereits an mehreren Experimenten teilgenommen. Dies bedingt, dass Erfahrungen aus vorangegangenen Experimenten als Hintergrundwissen auf nachfolgende Experimente einwirken. In diesem Sinne wirkt sich auch die Postsequenz der Auszahlung auf verschiedene Ebenen des Doings und der Realisierung der Experimente aus. Auf der Grundlage ihrer Vorerfahrungen bilden sich bei den ProbandInnen Erwartungen an den typischen Ablauf und den Inhalt der Experimente aus. Hinsichtlich des Doings einer spezifischen Laborsituation ist dieser Aspekt von Belang, da er sowohl die Reputation des Labors als auch die Glaubwürdigkeit der Zusagen und Aussagen der Forschenden positiv unterstützt. Für die teilnehmenden Versuchspersonen wird ersichtlich, dass die zugesagten Auszahlungen am Ende des Experiments tatsächlich realisiert werden und auch, dass die versprochene

\footnotetext{
${ }^{31}$ Es gibt jedoch auch Experimente, in denen Anonymisierungstechniken (siehe dazu Abschnitt 2.1.) eingesetzt werden, bei denen auch den ExperimentatorInnen verborgen bleibt, welche Versuchsperson welchen Spielgewinn erzielt hat. Es handelt sich dabei um doppelblind Versuche. In diesem Fall darf die Auszahlung nicht Face-to Face vorgenommen werden. Diese Art von Experimenten wurde jedoch nicht beobachtet.

${ }^{32} \mathrm{Zu}$ Abweichungen von der Barauszahlung am Ende des Experiments kann es kommen, wenn aufgrund des experimentellen Designs keine direkte Auszahlung vorgenommen werden kann. In einzelnen Fällen werden die Teilnehmenden auch mit geldwerten Gutscheinen entlohnt. Dies war jedoch in keinem der beobachteten Experimente der Fall, sondern nur Gegenstand der Schilderungen der befragten LaborforscherInnen.
} 
Anonymität der von ihnen gefällten Entscheidungen gegenüber den anderen ProbandInnen gewahrt bleibt. Für die Teilnehmenden besteht kein Grund, im Vollzug des Experiments bewusste Täuschungsversuche durch die Forschenden zu vermuten. Das institutionelle Setting der Universität, an welchem die Laborexperimente durchgeführt werden, und das soziale Milieu der Wissenschaft, welchem die Forschenden entstammen, bestärken dabei die Erwartung der ProbandInnen, dass die Auszahlung zum Abschluss des Experiments erfolgen wird.

Zugleich wird hier ersichtlich, wie die für das ökonomische Laboratop charakteristische Verbindung von Spielmodul und Ökonomiemodul im Vollzug des Experiments realisiert wird. $\mathrm{Zu}$ einer Auszahlung der erarbeiteten Gewinne kommt es nur, wenn sich die ProbandInnen im Vollzug des Experiments an die Regeln und Verhaltenserwartungen der Forschenden halten. Die Auszahlung stellt somit zum einen eine positive Sanktion für das regelkonforme Verhalten der Versuchsteilnehmer dar und trägt hinsichtlich nachfolgender Experimente zugleich zum Doing der rationalen und regelkonformen Verhaltenserwartungen bei. Wie eingangs beschrieben, erhalten nicht alle Teilnehmenden den gleichen Geldbetrag für ihre Teilnahme am durchgeführten Experiment. Wie in den Werbeplakaten, Instruktionen und Regeln mehrfach von Seiten der Forschenden dargestellt, erfolgt die Entlohnung der Teilnehmenden entscheidungsabhängig. Sie wird durch ihre Handlungen im Experiment sowie unter Umständen auch durch die Handlungen anderer ProbandInnen bestimmt. Unabhängig von den individuellen Ergebnissen erhält jeder Versuchsteilnehmer eine Minimalauszahlung von 5 Euro. In dieser Nutzung des monetären Anreizes ist dabei eine deutliche Orientierung an der induzierten Werttheorie Smiths (Abschnitt 5.2.2.2) ersichtlich, insbesondere an den Prinzipien der Monotonie und Salienz.

Die Auszahlung der erspielten Erträge ist zugleich ein zentrales Moment des Doings der spezifisch ökonomisch-rationalen Verhaltenserwartungen. In den Interviews berichteten alle befragten ProbandInnen, die bereits mehrmals an Experimenten teilgenommen hatten, dass ihre vorrangige Motivation zur Teilnahme in dem in Aussicht gestellten Geldgewinn bestand. Die Auszahlung der erspielten Gewinne stellt somit die Handlungsphase dar, auf welche das regelkonforme und strategische Handeln im Verlauf des Experiments ausgerichtet ist. Nur durch die Realisierung in der Phase der Auszahlung gelingt es somit, die monetäre Motivation im interaktiven Vollzug der Experimente als effektiven (positiven und negativen) Sanktionsmechanismus zu nutzen und auf diese Weise das Spielund das Ökonomiemodul zu verknüpfen.

Die beschriebenen Prä- und Postsequenzen der Ankunft und Auszahlung der ProbandInnen sind mit der Modulation des Laborgeschehens eng verknüpft und erweisen sich auch als prägend und einflussreich für den Rahmenbildungsprozess 
im Labor. Auch sie zeigen, wie dargestellt, charakteristische Mischungsverhältnisse der Spiel-, Experiment-, und Ökonomiemoduln. Die Anforderungen, die in diesen Prä- und Postsequenzen an regelkonformes Verhalten der ProbandInnen gestellt werden, sind jedoch weit geringer als im Labor. Sie markieren die zeitliche Klammer (siehe Abschnitt 3.1.3) der untersuchten Laborrahmung. Wie die Betrachtung der einleitenden Begrüßung (Abschnitt 5.3.3) aufzeigen wird, besteht die ,offizielle Klammer' in der datenerhebungsrelevanten Phase des Experiments. Sie beginnt mit der Vorstellung der zentralen Verhaltensregeln im Zuge der Einleitung. Das offizielle Ende des Geltungsbereichs der Laborrahmung mit seinen hohen Anforderungen an Regelkonformität bildet der Moment, an dem die ProbandInnen ihre Fragbögen ausgefüllt haben und das Labor verlassen.

\subsubsection{Standardisierter Prozess der Platzzuweisung}

Wie im Abschnitt 2.1 dargestellt, ist die Randomisierung ein zentrales Definitionskriterium des experimentellen Forschungsdesigns von Laborexperimenten. Den Annahmen der Experimentalforschenden folgend, ist sie das maßgebliche Verfahren, um den Einfluss bekannter und auch unbekannter Störvariablen zu minimieren, welche nicht durch das Konstanthalten der Randbedingungen ausgeschlossen werden können. Eine Randomisierung wird somit immer dann vorgenommen, wenn sich potenzielle Einflussfaktoren der direkten Kontrolle der Forschenden entziehen. Im Rahmen der Zuweisung von Arbeitsplätzen im beobachteten Labor erfolgt die empirische Umsetzung dieses Verfahrens mittels eines Spielchips. Wie oben dargestellt, ziehen die ProbandInnen vor dem Betreten des Laborraums einen kleinen nummerierten Spielchip. Die auf dem Spielchip befindliche Nummer weist den ProbandInnen einen Arbeitsplatz im Labor zu. An dieser Stelle wird noch einmal ein Ausschnitt aus dem einleitenden Analysebeispiel zur Illustration herangezogen (siehe Abschnitt 5.1). Hier expliziert der Experimentator Tim eben diesen Zusammenhang als Reaktion auf die Frage eines unidentifizierten Sprechers (Unid1):

Z 5: Unid1: und an die nummer setzen?

In Reaktion auf diese Frage antwortet Tim laut und für alle Wartenden deutlich hörbar: 


\section{Z 6: Tim: GENAU, AUF DEN PLATZ SETZEN (.) MIT DER} NUMMER AUFDEM CHIP

Nach einem unverständlichen Redebeitrag eines vor dem Labor befindlichen unidentifizierten Sprechers (Unid2):

\section{Z 7: Unid2: (...)}

Fügt Tim deutlich leiser hinzu:

Z 8: Tim: $>$ stehen an der wand $<^{\circ \circ}$

Dieser kurze Transkriptausschnitt verweist auf einen Zusammenhang zwischen drei Aspekten: Spielchip, Nummer an der Wand und dem Arbeitsplatz. Die Nummer auf dem gezogenen Spielchip zeigt den eintretenden ProbandInnen die Nummer ihres Arbeitsplatzes an. Die Nummerierung der Arbeitsplätze wiederum erfolgt durch Schilder, die an den Wänden innerhalb der Arbeitskabinen befestigt sind.

Durch das Ziehen des Spielchips entscheiden weder die Mitglieder des Laborteams noch die ProbandInnen, welcher Arbeitsplatz im Labor ihnen zugewiesen wird. Durch diesen zufälligen Zuweisungsmechanismus soll unterbunden werden, dass sich ProbandInnen aufgrund von sozialen Aspekten (wie Bekanntschaft, Sympathie oder Antipathie) gezielt neben bestimmte andere ProbandInnen im Labor setzen könnten oder der Prozess durch Mitglieder des Labors beeinflusst werden könnte. Ziel der Forschenden ist es, dem wissenschaftlichen Anspruch der Randomisierung von Einflussfaktoren im Labor auf diese Weise in anerkannter und standardisierter Art Rechnung zu tragen. Auch wenn die Zuordnung der ProbandInnen zu den einzelnen Arbeitsplätzen zufällig ist, kann durch die Mitglieder des Laborteams bestimmt werden, welche Arbeitsplätze im Labor besetzt werden und welche nicht. Dies erfolgt, indem sie vor dem Einlass der ProbandInnen festlegen, welche Zahlenchips im Beutel vorhanden sind. Das Vorgehen der zufälligen Sitzplatzzuweisung dient damit einer Ausschaltung unerwünschter ,sozialer' Störeffekte, die sich durch eine freie Platzwahl im Labor ergeben könnten. Diese Ethnomethode trägt damit zum Doing einer standardgerechten Experimentund Laborsituation bei. Auf diese Weise sollen wissenschaftlich adäquat die Standards der Durchführung von wirtschaftswissenschaftlichen Laborexperimenten und die Vergleichbarkeit der Ergebnisse gesichert werden. Die standardisierte Art der Platzzuweisung trägt so zur Verankerung des Experimentmoduls und zugleich zum Doing rationaler Verhaltenserwartungen gegenüber den ProbandInnen bei, da 
sie anzeigt, dass nicht die ProbandInnen darüber entscheiden, an welchen Laborarbeitsplatz sie sich setzen möchten. Es gehört vielmehr zu den Regeln des Spiels im Laborexperiment, dass sie der objektivierten Zuweisung durch den Spielchip Folge zu leisten haben.

Das Vorgehen der zufälligen Platzzuweisung stellt zugleich einen ersten Schritt der Anonymisierung dar. Auch das Mitglied des Laborteams, welches die ProbandInnen den Spielchip aus dem Beutel ziehen lässt, hat keinen Einfluss darauf, welche Versuchsperson welchen Arbeitsplatz im Labor einnehmen wird. Es wird hier für die Labormitglieder eine Entkopplung der personenbezogenen Daten der Eingeladenen von den Versuchspersonen an bestimmten Arbeitsplätzen initiiert. Die Zuordnung zu den Arbeitsplätzen ist somit anonym. Die zufällige Zuordnung wird nicht dokumentiert, sodass es im Nachhinein nicht mehr möglich ist, die persönlichen Daten der Versuchspersonen mit ihren Entscheidungen zu verknüpfen. ${ }^{33}$ Diese Art der Anonymisierung stellt somit ebenfalls einen Aspekt des Doings der spezifischen Laborsituation dar. Sie trägt wie auch die Randomisierung dazu bei, den wissenschaftlichen Annahmen folgend die Wirkung potenzieller und bekannter Störvariablen zu reduzieren, mit dem Ziel, bestmögliche Bedingungen für die Kontrolle und Isolation der hypothesenrelevanten Variablen zu schaffen.

In vielen Experimenten werden den Teilnehmenden in einer gegebenen Spielsituation zufällig unterschiedliche Rollen zugewiesen. ${ }^{34}$ Die eine Hälfte der Versuchsgruppe soll beispielsweise als Käufer, die andere Hälfte als Verkäufer agieren. Die Zuordnung zu bestimmten Rollen, die von einzelnen ProbandInnen eingenommen werden sollen, kann dabei durch verschiedene Arten erfolgen. Häufig ist sie ebenfalls durch die Arbeitsplätze bestimmt. Der Grund dafür besteht darin, dass bereits im Vorlauf der Experimente durch die Programmierung der Software festgelegt wird, an welchen Arbeitsplätzen die Teilnehmenden mit welchen Spielerrollen konfrontiert werden. Durch die zufällige Zuordnung zu den Arbeitsplätzen wird somit gleichsam erreicht, dass auch die Zuweisung von Rollen im Experiment zufällig erfolgt. Die Verteilung der ProbandInnen auf die Spielerrollen kann somit ebenfalls als randomisiert betrachtet werden. Ebenso wie bei der Zuweisung eines Arbeitsplatzes wird von den

\footnotetext{
${ }^{33}$ Es wäre allerdings eine zu starke Vereinfachung anzunehmen, dass die Mitglieder des Laborteams grundlegend keine Möglichkeit hätten, den Personen im Labor ihren personenbezogenen Daten zuzuordnen. Da es eine LabormitarbeiterIn ist, die vor dem Einlass ins Labor die Ausweisdokumente mit der Einladungsliste abgleicht, kann diese bei Interesse durchaus auch verfolgen, welchen Arbeitsplatz einzelne Personen im Labor einnehmen.

${ }^{34}$ Es gibt auch Experimente, in denen der Erwerb einer Rolle aktiv durch den Teilnehmenden erwirkt werden kann. Im nachfolgend geschilderten Beispiel eines Endowment-EffektExperiments wurde die Zuweisung der Käufer- bzw. Verkäuferrolle in einigen Treatmentvariationen beispielsweise durch das Gewinnen bzw. Verlieren eines Spiels bestimmt.
} 
ProbandInnen im Rahmen der Experimente erwartet, dass sie die ihnen zugewiesene Spielrolle an- und hinnehmen.

Es zeigt sich hier eine charakteristische Verknüpfung der Ebenen des Doings der Laborsituation mit jener des Doings der Verhaltenserwartungen gegenüber den ProbandInnen. Orientiert an den Prämissen der Methodologie des Experiments gilt es ein Umfeld für die Erhebung zu schaffen, welches Störfaktoren so weit wie möglich minimiert. Die Besonderheit der menschlichen Forschungssubjekte anerkennend sind jedoch gewisse Einflussfaktoren bekannt, die sich als Störfaktoren auf die Situation auswirken können. Sowohl die Vermutung einer Täuschung oder verdeckten Manipulation durch die Forschenden als auch soziale Verbindungen zwischen den ProbandInnen sollen als Störfaktoren minimiert werden. Das Verfahren der zufälligen Platzzuweisung ermöglicht eben dieses. Zugleich ist die Zufallsabhängigkeit ein typisches Moment der spezifischen Spielsituation im Labor, welches an vielfältigen Stellen in Erscheinung tritt. Zu den Regeln der Spielsituation des Laborexperiments gehört es, das Ergebnis des Zufalls anzuerkennen und im Fall der Zufallsziehung seine Handlungen daran zu orientieren. Das Verfahren der Platzzuweisung trägt somit sowohl zur Verankerung des Experimentmoduls als auch zur Verankerung des Spielmoduls bei und verweist zudem auf eine spezifische Verbindung, die zwischen diesen beiden besteht.

Der angesprochene Spielchip stellt dabei in seinen unterschiedlichen Funktionen zugleich ein Merkmal der Binnenstruktur und der situativen Realisierungsebene dar (siehe Abschnitt 5.4.1). Für den betrachteten Handlungsprozess stellt er im doppelten Sinne eine Objektivation bestimmter kommunikativer Handlungen und Wissensinhalte dar. Zum einen fungiert der Spielchip als eine Objektivation für den Prozess der Randomisierung und Anonymisierung. Er kann hier als Objektivation betrachtet werden, da sich in seiner Verwendung das Wissen und der Anspruch der Forschenden ausdrückt, die Platzzuordnung der Teilnehmenden auf eine ganz spezifische Art zu koordinieren und zu kontrollieren. Gerade die besondere Performanz und die Performativität, mittels welcher das Verfahrens umgesetzt wird, ist von entscheidender Bedeutung für seine Wirkung. Sie sind, wie oben dargestellt, Ausdruck der Unbeeinflussbarkeit und des Zufalls des Ergebnisses. Für die teilnehmenden ProbandInnen bietet der Zahlenchip darüber hinaus eine Objektivation für das situativ notwendige Wissen, welches sie zur interaktiven Handlungskoordination im Labor benötigen. Mittels des nummerierten Spielchips ist es ihnen möglich, das Handlungs- und Entscheidungsproblem zu lösen und zu bestimmen, welchen Arbeitsplatz im Labor sie einnehmen sollen. Dieser Aspekt wird auf Ebene der situativen Realisierung im Abschnitt 5.4.1 thematisiert. 


\subsubsection{Einleitende Begrüßung und Kommunikation der Verhaltensregeln}

Nachdem alle ProbandInnen das Labor betreten und ihre Sitzplätze eingenommen haben, kann der Übergang zur Handlungsphase der Begrüßung erfolgen. $\mathrm{Zu}$ diesem Zeitpunkt befindet sich zudem mindestens eine ExperimentatorIn im Labor. Den Auftakt dieser Handlungssequenz markiert ein Mitglied des Laborteams, welches laut und deutlich zu sprechen beginnt und die Teilnehmenden im Labor willkommen heißt. Der Inhalt dieser Begrüßung weist eine deutliche Standardisierung auf. Es erfolgt hier die wiederholte Vorstellung der Regeln, denen die ProbandInnen auch schon bei der Anmeldung für den Pool der ProbandInnen zustimmen mussten. Den Versuchspersonen wird erneut mitgeteilt, dass die Nutzung von Smartphones oder anderen technischen Geräten während des Experiments untersagt ist. Zudem dürfen sie nur die für das Experiment vorgesehenen Programme des Computers nutzen. Die ProbandInnen werden darüber informiert, dass es ihnen außerdem nicht gestattet ist, miteinander auf irgendeine Art und Weise zu kommunizieren. Als dritten Aspekt werden sie darüber belehrt, dass Fragen von Teilnehmenden auf keinen Fall laut gestellt werden dürfen. Sollte eine TeilnehmerIn eine Frage haben, so wird er/sie gebeten, die Hand zu heben. Ein Mitglied des Laborteams werde in diesem Fall zu ihnen kommen, um die Frage individuell und leise zu beantworten. Im Anschluss an diese Belehrung werden die ProbandInnen darauf hingewiesen, dass ein Zuwiderhandeln gegen diese Regeln zu einem Ausschluss vom Experiment und zum Verlust all ihrer bisher erspielten Gewinne führen kann. Nach Abschluss dieser einleitenden Belehrung werden die ProbandInnen dazu aufgefordert, mit dem Lesen der Instruktionen zu beginnen.

Der typische Ablauf und Inhalt der Begrüßungssequenz trägt auf verschiedenen Ebenen des Doings deutlich zur kommunikativen Konstruktion des Laboratops bei. Im Zuge dieser einleitenden Belehrung wird die Relevanz der Regelbefolgung gegenüber den Teilnehmenden noch einmal explizit kommuniziert. Das erneute Kommunizieren der Verhaltensregeln des Labors und damit der angestrebten Handlungsrestriktionen der ProbandInnen ist als performative Strategie zu verstehen. Sie dient als Ethnomethode dem Doing regelkonformen Verhaltens und bildet zugleich die ,offizielle' zeitliche Klammer für das Spielmodul. Ab jetzt wird von den ProbandInnen die Befolgung der durch die Belehrung explizierten Spielregeln erwartet. Das Spielmodul tritt hier als charakteristisches Rahmungselemente deutlich in Erscheinung.

Mit dem Vollzug der einleitenden Belehrung wird zugleich ausgewiesen, welche soziale Rolle mit welchen Rederechten im Labor verbunden ist. Während ein Mitglied des Laborteams das Wort ergreift und damit den Auftakt zu einer neuen 
Handlungssequenz markiert, wird den ProbandInnen wiederholt deutlich mitgeteilt, dass es ihnen strikt untersagt ist, offen zu kommunizieren. Dieses Verbot bezieht sich sowohl auf jedwede Kommunikation mit anderen ProbandInnen als auch auf das offene und laute Stellen von Fragen. Die einzig offiziell zulässige und sanktionsfreie Möglichkeit der ProbandInnen, sich verbalsprachlich zu entäußern, besteht darin, in einem leisen Zwiegespräch mit einer ExperimentatorIn eine Frage zu stellen. Diese Option steht den VersuchsteilnehmerInnen jedoch nur offen, wenn sie zuvor auf die geforderten Ethnomethoden zurückgreifen, sich per Handzeichen melden und darauf warten, dass ein Mitglied des Laborteams zu ihrem Arbeitsplatz kommt. ${ }^{35}$ Den Mitgliedern des Laborteams hingegen obliegt es, darüber zu entscheiden, ob die gestellten Fragen so relevant sind, dass sie allen Teilnehmenden gegenüber wiederholt und offen beantwortet werden.

Die Performanz der einleitenden Belehrung bildet dabei selbst eine Objektivierung der Eingriffs- und Handlungsrechte, die mit den unterschiedlichen sozialen Rollen im Labor einhergehen. Der Auftakt und auch das Ende dieser Handlungssequenz werden hier durch den Experimentator initiiert und markiert. Sowohl inhaltlich als auch performativ ist das kommunikative Handeln der einleitenden Begrüßung Ausdruck von Kommunikationsmacht. ${ }^{36}$ Es markiert eine deutliche Machtasymmetrie der beteiligten Akteursgruppen hinsichtlich ihrer legitimen Einflussnahme auf die Handlungsvollzüge im Labor. Die Kontrolle über die Handlungsabläufe im Labor wird den ExperimentatorInnen zugeschrieben und nicht den VersuchsteilnehmerInnen. Mittels der einleitenden Belehrung wird kommuniziert, dass es zu den Spielregeln des ökonomischen Laboratops gehört, die unterschiedlichen Handlungsspielräume zwischen den Mitgliedern des Laborteams und den VersuchsteilnehmerInnen anzuerkennen. Dieses binnenstrukturelle Merkmal trägt somit zum Doing regelkonformer Verhaltenserwartungen gegenüber den ProbandInnen bei und zur Verankerung des Spielmoduls. Das Accounting, in denen sich die Anerkennung und das Verständnis dieser Regeln materialisiert, wird durch die Bezugnahme auf eben diese im weiteren Prozess des Laborexperiments angezeigt.

Durch seine Standardisierung unterstützt die Begrüßung das Doing einer spezifischen Experimentsituation. Die drei zentralen Regeln im Labor stellen dabei Aspekte dar, die als potenzielle Störeinflüsse betrachtet werden. Die Belehrung über die Regeln der Experimente zielt dabei auf das Ausschalten dieser

\footnotetext{
${ }^{35}$ In der nachfolgenden Analyseebene der situativen Realisierung wird auf den Aspekt des Fragestellens im Abschnitt 5.4.3 noch detaillierter eingegangen.

${ }^{36}$ Der Begriff Kommunikationsmacht wird hier an Reicherts (2009) orientiert genutzt. Nähere Ausführungen zu diesem Konzept finden sich im Abschnitt 5.3.2.
} 
potenziellen Einflussfaktoren ab und soll eine Kontrolle über die Informationsgrundlage ermöglichen, die den ProbandInnen in den Versuchen zur Verfügung steht. Wie die bisherigen Beispiele bereits zeigten, wird die Standardisierung als eine zentrale Methode betrachtet, um gleichartige Situationen der Datenerhebung zu produzieren und so dem wissenschaftlichen Anspruch des Konstanthaltens der Randbedingungen zu entsprechen.

Durch den abschließenden Verweis auf den in Ausschicht gestellten monetären Gewinn bzw. dessen Verlust sollen zugleich Anreize geschaffen werden, welche ein Handeln der ProbandInnen im gewünschten Sinne der Forschenden begünstigen. Indem als Sanktion für potenzielle Regelüberschreitungen auf die Gefahr des Verlustes der erarbeiteten Gewinne verwiesen wird, zeigt sich erneut der praktische Zusammenhang zwischen dem Doing regelkonformen Verhaltens und dem Einsatz des monetären Anreizes als Beitrag zu einem Doing ökonomisch-rationaler Verhaltenserwartungen. Ganz im Sinne des ökonomischen Akteursmodells werden die Akteure hier über die Restriktionen ihres Handlungsspielraums in Kenntnis gesetzt. Auch hier wird wieder auf den monetären Anreiz für den Fall der Zuwiderhandlung verwiesen. Smiths induzierte Werttheorie (Abschnitt 5.2.2.2) findet in eben diesem Vorgehen ihren Ausdruck, indem der finanzielle Anreiz eingesetzt wird, um zu einem regelkonformen Verhalten zu motivieren.

Im Zusammenspiel von Spiel-, Experiment-, und Ökonomiemodul zeigt sich hier, wie diese Modulationen verschachtelt ineinander verankert werden. Die Begrüßung selbst bildet die Klammer, welche den Beginn der Modulation eindeutig markiert und verankert. Das Grundmodul bildet hier das Spielmodul. Es werden die allgemeinen Regeln der Spielsituation eingeführt sowie die unterschiedlichen allgemeinen Spielergruppen (ProbandInnen und ExperimentatorInnen) und die erlaubten allgemeinen Züge, die diese regelkonform vornehmen dürfen. Orientiert am wissenschaftlichen Standard der experimentellen Methodologie und dem mikroökonomischen Forschungsinteresse werden dabei die Regeln spezifiziert, die hier allgemeine Geltung beanspruchen. Es wird ausgewiesen, dass eine Standardisierung der Erhebungssituation (Experimentmodul) und eine gleichartige Informationsgrundlage für alle Versuchsteilnehmenden angestrebt wird. Durch die kommunikative Vereinzelung der ProbandInnen werden dabei die Vorbedingungen für die standardisierte und kontrollierte Erhebung ihres individuellen ökonomischen Entscheidungsverhaltens (Ökonomiemodul) geschaffen. 


\subsubsection{Instruktionen als Mittel der Situationsdarstellung}

Die Instruktionen stellen ein zentrales Element des Spielmoduls dar, mittels welchem den VersuchsteilnehmerInnen die Regeln des spezifischen Experiments, die Spielsituation, die Rollen und die Gewinnmöglichkeiten und -modalitäten präsentiert werden. Auch wenn die inhaltlichen Fragestellungen der beobachteten Experimente variieren, lassen die experimentspezifischen schriftlichen Instruktionen im Vergleich auch deutliche Gemeinsamkeiten erkennen. Zu Beginn folgt stets eine einleitende Belehrung über die Regeln des Experiments. Im Anschluss werden den VersuchsteilnehmerInnen die Entscheidungssituation und die Rollen, die sie zugewiesen bekommen können, vorgestellt und ausführlich beschrieben. Zudem wird erläutert, nach welchem Zuweisungskriterium sie die entsprechenden Rollen einnehmen werden, das heißt, ob die Zuweisung beispielsweise zufällig erfolgt oder aufgrund eines vorherigen Ereignisses wie dem Gewinn in einem Spiel. ${ }^{37}$ Im Zuge der Instruktionen werden ausführlich die spezifischen Regeln erklärt, nach denen sich entscheidet, welche Handlungen zu welchen Gewinnmöglichkeiten für die Teilnehmenden führen können. ${ }^{38}$ In vielen Experimenten werden mehrere Runden eines Spiels von den Teilnehmenden nacheinander absolviert. Die Versuchspersonen werden in diesen Fällen in den Instruktionen zusätzlich über die spezifischen Gewinnmodalitäten informiert. Es wird den ProbandInnen beispielsweise mitgeteilt, ob sich ihre Gewinne über mehrere Runden hinweg akkumulieren oder am Ende des Experiments einzelne auszahlungsrelevante Runden zufällig ausgewählt werden. In den Instruktionen wird zudem dargestellt, welche Konsequenzen bestimmte Handlungsoptionen haben können. Können in einem Experiment Punkte oder Tokens verdient werden, so wird den VersuchsteilnehmerInnen darüber hinaus mitgeteilt, zu welchem, Wechselkurs' diese am Ende des Experiments in Bargeld umgerechnet werden. Durch die Darstellung dieser Spielregeln wird zum Doing der ökonomisch-rationalen Verhaltenserwartungen beigetragen. Da das ökonomische Entscheidungsverhalten den Forschungsgegenstand der experimentellen Wirtschaftsforschenden bildet, sind die Experimente inhaltlich auf eben diese ausgerichtet. Es wird somit kommuniziert, dass es sich bei dem folgenden Spiel um eines aus der Welt des ökonomischen Entscheidens handelt soll. Das allgemeine Ausweisen der

\footnotetext{
${ }^{37}$ Der Aspekt der zufälligen Zuweisung von Spielerrollen durch die Arbeitsplatznummer wurde in 5.3 .2 bereits angesprochen.

${ }^{38}$ Dies entspricht dem Aspekt der entscheidungsabhängigen Entlohnung. Nicht die Teilnahme der ProbandInnen, sondern ihre Handlungen im Experiment und in manchen Experimenten auch die Handlungen anderer ProbandInnen sind ausschlaggebend für die Höhe des Geldbetrages, welchen sie am Ende des Experiments erhalten können.
} 
Gewinnmöglichkeiten und -modalitäten sowie die Inhalte der Experimente selbst verbinden hier zugleich das Spiel- und das Ökonomiemodul.

Gerade der Beginn der Instruktionen weist eine deutliche Standardisierung der dargestellten Inhalte auf. Es erfolgt dabei eine Wiederholung der Regeln des Experiments, die bereits einige Augenblicke zuvor in der Begrüßung kommuniziert wurden. In den internen Regeln des beobachteten Labors wurde im Verlauf des Projekts bindend der Standardbeginn für die Instruktionen festgelegt. Alle Forschende, die Experimente im Labor durchführen, wurden gebeten ihre Instruktionen mit folgendem Passus zu beginnen:

„Herzlich Willkommen bei unserem Experiment!Während des Experimentes ist es Ihnen nicht erlaubt elektronische Geräte zu benutzen oder mit anderen Teilnehmern zu kommunizieren. Bitte benutzen Sie nur die für das Experiment vorgesehenen Programme und Funktionen. Bitte sprechen Sie nicht mit den anderen Teilnehmern. Sollten Sie eine Frage haben, dann heben Sie bitte Ihre Hand. Wir werden dann zu Ihnen kommen und Ihre Frage im Stillen beantworten. Bitte stellen Sie Ihre Fragen auf keinen Fall laut. Wenn die Frage relevant für alle Teilnehmer ist, werden wir sie laut wiederholen und beantworten. Sollten Sie gegen diese Regeln verstoßen, müssen wir Sie vom Experiment und der Auszahlung ausschließen. “39

Die gezielte und mehrfache Wiederholung von relevanten Inhalten ist eine typische performative Ethnomethode in der Durchführung von Experimenten. Von Seiten der Forschenden wird dabei, in Anlehnung an die einschlägigen Standardwerke zur Durchführung von wirtschaftswissenschaftlichen Experimenten (z. B. Sunder \& Friedman 1994), angenommen, dass eine gezielte Wiederholung und Doppelung von zentralen Aspekten das Regelverständnis der Teilnehmenden fördert. Die Umsetzung dieser Annahme zeigt sich hier deutlich am Beispiel der zentralen Regeln des Experiments. Diese werden nicht nur an mehreren Gliederungspunkten der durchgeführten Experimente wiederholt, sondern, wie aus dem Standardbeginn der Instruktionen ersichtlich, auch noch einmal selbst paraphrasiert und dabei nur geringfügig spezifiziert.

In Zuge der Instruktionen werden die ProbandInnen zudem häufig explizit auf die Anonymität der ihren zugewiesenen Rollen und der von ihnen getroffenen Entscheidungen hingewiesen. Wie in Abschnitt 2.1 ausgeführt, zielen die Techniken

\footnotetext{
${ }^{39}$ Zitiert aus einem internen Dokument „Lab Rules“.
} 
der Anonymisierung primär darauf ab, den potenziellen Einfluss von experimentinternen, interaktionsbedingten Störfaktoren wie sozialen Erwünschtheitseffekten auf die Datenerhebung zu unterbinden. Es handelt sich somit um Verfahren, die zur Minimierung von potenziellen Störeinflüssen beitragen. In dieser Hinsicht sind Anonymisierungstechniken in ihrer empirischen Umsetzung Aspekte des Doings einer spezifischen Laborsituation. Um die Einhaltung der Anonymisierung den ProbandInnen zu kommunizieren und zu verdeutlichen, ist sie häufig auch ein inhaltlicher Aspekt der Instruktionen. ${ }^{40} \mathrm{Im}$ Zuge einer beobachteten Serie von Experimenten zum Thema Mindestlohn wurde dies beispielsweise durch folgenden Passus in den Instruktionen ausgedrückt:

„Zu Anfang des Experiments wird jedem Teilnehmer zufällig eine Rolle zugeordnet, die bis zum Ende des Experiments die gleiche bleibt. Sie kennen Ihre eigene Rolle, wissen aber nicht, welche Rollen die anderen Teilnehmer einnehmen. Selbstverständlich bleibt Ihre Anonymität während des gesamten Experiments gewahrt. Das heißt, dass die anderen Experimentteilnehmer Ihre wirkliche Identität nicht erfahren. Das gleiche gilt für alle Teilnehmer. "41

Es wird hier explizit darauf aufmerksam gemacht, dass keiner der anderen Anwesenden weiß, welche Rolle einzelne ProbandInnen in einem Experiment einnehmen. Dies geht damit einher, dass auch die von ihnen getroffen Entscheidungen von anderen ProbandInnen nicht auf sie zurückgeführt werden können.

Aus Sicht der experimentellen Wirtschaftsforschenden führen die Instruktionen in die Entscheidungssituation ein und bieten den ProbandInnen die Informationsgrundlage, die sie für ihre Handlungen und Wahlentscheidungen heranziehen. Auf dieser Grundlage sollen die ProbandInnen zudem ihre Strategien zur Bearbeitung

\footnotetext{
${ }^{40}$ Bereits im Zuge der Rekrutierung werden die zukünftigen ProbandInnen darüber informiert, dass ihre Teilnahme am Experiment anonym ist. In den zur Verfügung gestellten Informationsmaterialien wird dargestellt, dass im Experiment erhobene „Entscheidungsdaten“ in der Auswertung anonymisiert werden und nachträglich keiner Person mehr zugeordnet werden können. Diese typisch wissenschaftliche Anonymisierung im Zuge der Datenauswertung ist jedoch für den hier thematisierten Zusammenhang nur indirekt relevant. Die Entkopplung von personenbezogenen Daten und Entscheidungsdaten wird in einem ersten Schritt durch die Randomisierung und das Ziehen des nummerierten Spielchips im Labor realisiert (siehe Abschnitt 5.3.2 und 5.4.1).

${ }^{41}$ Quelle: Auszug aus den Instruktionen der Experimentserie „Mindestlohn“
} 
eben dieser Situationen entwickeln können. Da die Instruktionen von den Forschenden verfasst werden, gehen sie davon aus, dass sie somit Kontrolle über die Informationsgrundlage und über den Orientierungsrahmen der Entscheidungssituation der ProbandInnen besitzen. Die Instruktionen zielen somit darauf ab, zentrale Elemente der angestrebten Situationsrahmung zu formulieren. Sie geben das Gerüst des kleinen mikroökonomischen Kosmos vor, das in der Spielsituation des jeweiligen Experiments als Bezugsrahmen angenommen werden soll. Sie zielen darauf ab, das System der Konventionen zu kommunizieren, welches das spezifische Spielmodul des jeweiligen Experiments charakterisiert.

Die Instruktionen selbst können als kommunikative Kleinform betrachtet werden: Sie beinhalten typische sprachliche und inhaltliche Merkmale. Das Medium dieser Vermittlung kommunikativer Handlungen ist der maschinell auf Papier geschriebene Text. Es gilt somit den Schritt von der Formulierung der Rahmenaspekte, an denen sich die ProbandInnen orientieren sollen, zur Indizierung eben dieser zu bewerkstelligen. Für die Forschenden ist es dabei von entscheidender Bedeutung, dass die ProbandInnen die Regeln des Spiels in der von ihnen antizipierten Weise verstehen, da nur so die interne Validität des Experiments gesichert werden kann. Das Ziel der Instruktionen besteht stets darin, den VersuchsteilnehmerInnen die Regeln des Experiments in einer Weise darzustellen, die es ihnen ermöglicht, sie in der von den Forschenden angestrebten Weise zu verstehen.

Die Darstellungskonventionen, an denen sich die Forschenden orientieren, sind eine Ethnomethode, mittels derer das Sprachspiel der Forschenden zu dem der Beforschten gemacht werden soll. Dies bedeutet dabei nicht, dass notwendigerweise ökonomische Fachbegriffe wie „Nutzentheorie“ und „Erwartungswert“ oder die Benennung bestimmter Mechanismen in den Instruktionen zu finden sind. ${ }^{42}$ Durch die Anerkennung der Erkenntnisse über Framing und Framing-Effekte in den Studien von Kahneman und Tversky (Abschnitt 5.2.2.1) sind die Forschenden sensitiv gegenüber der Wirkung semantischer Effekte. Es wird aus diesem Grund angestrebt, in der Darstellung der Spielsituation des Experiments eine ,neutrale Sprache‘ zu nutzen, die bei den VersuchsteilnehmerInnen keine unerwünschten Demand-Effekte verursacht. Welche Begriffe oder Darstellungen von Zusammenhängen als problematisch oder adäquat gelten, ist dabei deutlich an der wissenschaftlichen Peergroup orientiert. Der sprachliche Code der ökonomischen Laborexperimente tritt in den Instruktionen deutlich in Erscheinung.

\footnotetext{
${ }^{42}$ Einige Begriffe, die häufig in Instruktionen auftreten, haben jedoch eine spezifisch ökonomische Konnotation, die von ihrer alltagsweltlichen Verwendung deutlich abweicht. Der Begriff der „Lotterie“ ist hier ein passendes Beispiel. Wie weiter unten noch dargestellt wird, hat dieser Begriff im Sprachspiel der experimentellen WirtschaftsforscherInnen recht wenig mit dem Lottospielen und der Sonntagsziehung der Gewinnzahlen zu tun.
} 
Dieser zeigt sich in den Instruktionen als charakteristischer Einsatz von Begriffen, die die Spieldimension und den Bereich wirtschaftlichen Handelns betonen und so die Verankerung des Spiel- und Ökonomiemoduls unterstützen. So werden, neben der allgemeinen Bezeichnung von ProbandInnen als „Teilnehmer“, beispielsweise häufig Begriffe wie „Spieler“, „Gegenspieler“, „Käufer“, „,Verkäufer" zur Charakterisierung und Differenzierung der unterschiedlichen Rollen der ProbandInnen genutzt. In Fällen, in denen das Ziel der Experimente expliziert wird, wird zumeist als eine Untersuchung von „Entscheidungsfindung“ oder „Entscheidungsverhalten" bezeichnet. Hier wird sprachlich festgelegt und damit ganz im Sinne der „dokumentarischen Methode“ (Garfinkel 1973: 209) ,festgestellt“, dass die Handlungen, die die ProbandInnen im Verlauf der Experimente vollziehen, als Entscheiden bezeichnet werden können. Zugleich wird darauf verwiesen, dass die Messbarmachung ${ }^{43}$ dieses Entscheidens durch die im Labor eingesetzten Techniken und Verfahren möglich ist. Auch die Aktionen, die die ProbandInnen in diesen Situationen vollziehen sollen, werden sprachlich so gerahmt, dass sie die Verknüpfung von Spiel- und Ökonomiemodul und zugleich die Dimension des strategischen Handelns ausdrücken. Es geht um „Strategien“ und es soll „gespielt“, ,,entschieden“, „gekauft“, „,verkauft" werden. Die Instruktionen weisen dabei ein übliches Spannungsverhältnis zwischen der Verständlichkeit für die ProbandInnen, der Vollständigkeit der Darstellung für Berichtszwecke und den Anforderungen der Referees auf (vgl. auch Friedman \& Sunder 1991: 53).

Ein Grundsatz der experimentellen Wirtschaftsforschung im Labor ist, dass die Teilnehmenden bei Experimenten nicht getäuscht werden dürfen. Dies gilt sowohl für schriftliche Instruktionen als auch für Inhalte, die ein Mitglied des Laborteams während des Experiments kommuniziert. Dies meint nicht, dass die ProbandInnen über alle Ziele und Spezifika des experimentellen Designs informiert werden müssen. Die gezielte Vermittlung von falschen und irreführenden Informationen oder Zielsetzungen ist jedoch strengstens untersagt. Mit der Anmeldung zur Durchführung von Experimenten im untersuchten Labor stimmen die Forschenden zu, sich an diese Regeln zu halten. Wie im Zusammenhang mit den sozialen Rollen der ExperimentatorInnen dargestellt (siehe Abschnitt 5.2.3.1), zielt dieses Täuschungsverbot darauf ab, die Reliabilität der im Labor durchführten Datenerhebungen zu bewahren und damit den Einfluss von vermeidbaren Störvariablen zu reduzieren.

Da alle ProbandInnen im Labor stets gleiche schriftliche Instruktionen erhalten, stellen diese ein Moment des Doings einer spezifischen Experimentalsituation und - bezogen auf das Konstanthalten von Randbedingungen - einen Aspekt

\footnotetext{
${ }^{43}$ Ein maßgeblicher Schritt zur ,Messbarmachung ' des Entscheidens wird durch den Einsatz technischer Medien erreicht. Abschnitt 5.3.8 widmet sich diesem Aspekt.
} 
der Verankerung des Experimentalmoduls dar. Diese Gleichartigkeit der genutzten Instruktionen besteht dabei jedoch nicht nur auf der Ebene der in einem Labor anwesenden ProbandInnen, sondern auch über einzelne Sessions hinweg. Wie oben beschrieben, werden für die Datenerhebung einer Versuchsanordnung (Treatmentvariation) üblicherweise mehrere Sessions im Labor durchgeführt, um eine ausreichend große Fallzahl für die statistische Analyse zu generieren. In allen durchgeführten Sessions einer Treatmentvariation werden dabei die gleichen Instruktionen verwendet. Im Sinne des angestrebten Parallelgruppenmodells (siehe Abschnitt 2.1) werden zudem im Zuge einer Serie von Experimenten stets mehrere Treatmentvariationen durchgeführt. Die Differenz zwischen den unterschiedlichen Instruktionen, die in verschiedenen Treatmentvariationen eingesetzt wird, soll dabei so gering wie möglich gehalten werden. Die Veränderung zwischen den Versuchsanordnungen soll so fokussiert wie möglich erfolgen, um in der nachfolgenden Kausalanalyse eindeutige Schlüsse bezüglich der Veränderung der Ergebnisse zu erlauben. Damit einher geht das Bestreben, alle anderen Aspekte konstant zu halten. Die Verwendung standardisierter Instruktionen verankert das Experimentmodul somit nicht nur in einer einzelnen Session, sondern erstreckt sich über diese hinaus.

\subsection{5 Überprüfung des Text- und Regelverständnisses}

In der Praxis der wirtschaftswissenschaftlichen Laborforschung werden spezifische Methoden eingesetzt, um das Text- und Regelverständnis der TeilnehmerInnen zu überprüfen. Es soll so die Chance erhöht werden, dass das Sprachspiel der Forschenden und die damit einhergehende Binnenlogik der Spielsituation auch von den ProbandInnen übernommen wird. Ein Beispiel ist der Einsatz von Testfragebögen. Nachfolgender Interviewausschnitt zeigt auf, wie die ProbandInnen diesen Handlungsaspekt verstehen und wie er typischerweise in den Verlauf des Experiments eingebaut ist:
Roland:
Manchmal gibt's dann auch so ein Verständnistest.
Dennis: Obwohl nicht immer.
Roland: Ja manchmal, stimmt, den hatte ich aber schon seit drei-, viermal nicht mehr, aber ansonsten noch ein Verständnistest.
Interviewerin: Wie ist das mit dem Test? Was passiert da? 
Roland: $\quad$ Da werden so Fragen meistens Multiple Choice gestellt, um zu überprüfen, ob man die Aufgabe verstanden hat oder nicht, weil die manchmal ein bisschen vertrakt sind, mit vielen unterschiedlichen Koordinaten sage ich jetzt mal und da kann es manchmal ein bisschen verwirrend werden, wollen die kurz, wollen die aber wissen, ob man das jetzt kapiert hat oder nicht.

Interviewerin: Okay, und die werden dann eingesammelt?

Dennis: $\quad$ Oder abgehakt je nachdem. Also meistens ist es so, dass man die eben versucht zu lösen oder löst und dann kommt jemand rum, guckt, ob alles richtig ist, also ob man es dementsprechend verstanden hat. Dann, wenn jeder das geschafft hat, dann fängt's an.

Roland: $\quad$ Oder wenn eben jemand das nicht geschafft hat, dann erklären sie die einzelnen Punkte noch mal je nachdem.

In einigen beobachteten Fällen wurde nach dem Lesen der Instruktionen ein Verständnistest mit den Teilnehmenden durchgeführt, um zu überprüfen, ob sie die Instruktionen in der von den Forschenden antizipierten Weise verstanden hatten. In diesen Fragebögen wurden exemplarisch Entscheidungssituationen nachgestellt, die dem nachfolgenden Experiment entsprachen. Häufig handelte es sich um eine Reihe von Multiple-Choice Fragen, welche die ProbandInnen durch ankreuzen auf einem Papiervordruck beantworten mussten. Traten vermehrt gleichartige „Fehler“ auf, so wurden relevante Aspekte noch einmal durch die ExperimentatorInnen erklärt.

Die Darstellung ausführlich erörterter Beispiellösungen in den Instruktionen oder die verbalen Erklärungen der ExperimentatorInnen sind weitere Möglichkeiten, um den TeilnehmerInnen nahezulegen, welche Art des Verständnisses der Spielregeln während des Experiments von ihnen erwartet wird. Zugleich illustrieren diese Beispielfälle und Erklärungen auch, welche Handlungen in den Experimenten zu welchen Resultaten und damit zu welchen Spielgewinnen für die ProbandInnen führen können. Dadurch vollzieht sich nicht nur ein Doing regelkonformer Verhaltenserwartungen, sondern es wird zugleich auch zum Doing von spezifisch ökonomischen Verhaltenserwartungen beigetragen.

$\mathrm{Ob}$ Verfahren zur Überprüfung und Verbesserung des Regelverständnisses eingesetzt werden, ist dabei keine Entscheidung, die situativ durch die ExperimentatorInnen getroffen wird. Es ist vielmehr so, dass solche Entscheidungen im experimentellen Design der Studien angelegt sind. In allen durchgeführten Sessions einer 
Treatmentvariation wird dieses Vorgehen auf standardisierte Weise angewendet. Auch hier wird wieder dem Credo des Konstanthaltens der Randbedingungen gefolgt, welches eine maximale Standardisierung aller Prozesse anstrebt.

Die schriftlichen und verbalen Instruktionen stellen einen Aspekt des Doings regelkonformen Verhaltens der ProbandInnen dar. Dies verhilft zur Verankerung des Spielmoduls und zugleich zu seiner Spezifizierung in der jeweiligen Treatmentvariation. Den ProbandInnen werden die Regeln des Spiels vorgestellt und die Informationen präsentiert, die sie als Grundlage für ihre Entscheidungen heranziehen sollen. Bestimmte Begriffe sollen auf eine spezifische Art und Weise verstanden werden und es erlauben, eine gezielte Informationsgrundlage für die nachfolgenden Entscheidungssituationen und Verhaltensspiele zu schaffen. Dass die ProbandInnen diese Informationsgrundlage in der von den Forschenden antizipierten Weise verstehen, wird durch die beschriebenen Techniken der Forschenden gefördert.

\subsubsection{Lotterien}

Der Einsatz spezifischer Mechanismen und Verfahren, welche das Entscheidungsverhalten von ProbandInnen messbar machen (sollen), ist charakteristisch für das wirtschaftswissenschaftliche Experimentieren. Sie sind Ausdruck der paradigmatischen Annahmen der wirtschaftswissenschaftlichen ForscherInnen und des Denkkosmos der Wissenschaftscommunity, dem die jeweiligen Forschenden entstammen. ${ }^{44}$ Das Verständnis dieser Mechanismen und Verfahren ist recht voraussetzungsvoll, da es an spezifische wirtschaftswissenschaftliche Wissensbestände gekoppelt ist. Die Art und Weise ihres Einsatzes ist prägend für die Herausbildung und Verankerung des Ökonomiemoduls und spezifiziert und verankert zugleich das Spielmodul. Die konkrete Art der Spiele, die im Zuge einzelner Experimente gespielt werden sollen, und die charakteristische Form der Informationsvermittlung, die Forschende als die relevante Entscheidungsgrundlage der ProbandInnen erachten, zeigt sich in der Präsentation und Anwendung dieser Verfahren. Anhand der Thematisierung von Lotterien soll nachfolgend aufgezeigt werden, wie wirtschaftswissenschaftliche Modellannahmen und Konzepte Eingang in die Modulation der sozialen Situation finden und so das Spiel- und Ökonomiemodul mit dem Experimentmodul verbinden. Die wissenschaftliche Messbarkeit ökonomischen Entscheidungsverhaltens wird durch den Einsatz eben

\footnotetext{
${ }^{44}$ Gleiches gilt für alle anderen wissenschaftlichen Disziplinen. Auch ich als Soziologin bin in der Auswahl meiner Verfahren und Methoden für meine Datenerhebung von paradigmatischen Annahmen geprägt. Von Interesse für die vorliegende Untersuchung ist jedoch die Frage, wie die Umsetzung und der Einsatz dieser Verfahren die Rahmung der Laborsituation prägt.
} 
solcher Verfahren zum Inhalt der Entscheidungsspiele gemacht, mit denen die ProbandInnen sich auseinandersetzen müssen.

Entscheidungen unter unsicheren Bedingungen werden in mikroökonomischen Untersuchungen oft als Lotterien modelliert. Lotterien bilden Entscheidungen unter Risiko ab. Auch wenn die Eintrittswahrscheinlichkeiten für die einzelnen Handlungsalternativen bekannt sind, kann der Nutzen der einzelnen Wahlalternativen von den handelnden Akteuren nicht unmittelbar festgelegt werden. Der Grund liegt darin, dass die Ergebnisse erst ausgespielt werden müssen, um festzustellen, ob der Akteur bei einer Lotterie gewinnt oder verliert. Statt des Nutzens einer Lotterie (Wahlalternative) wird in der ökonomischen Konzeption aus diesem Grund der Erwartungswert als der Nutzen einer Lotterie bestimmt, welcher die Alternativen in Kombination mit ihren Eintrittswahrscheinlichkeiten bewertet. Orientiert an der Spieltheorie wird der Einsatz von Lotterien und die Wahl zwischen diesen als eine strategische Spielsituation verstanden. Die ProbandInnen müssen sich hier als Spieler für eine Handlungsalternative entscheiden. Als ihre Gegenspielerin kann dabei die Wahrscheinlichkeitsverteilung der Lotterie selbst verstanden werden. Die Lotterien stellen die Informationen zur Verfügung, die die ProbandInnen bei ihrer Auswahl zwischen den unterschiedlichen Handlungsalternativen oder der Bewertung des Preises eines Lotterietickets heranziehen sollen.

Die unterschiedlichen Auswahlalternativen werden hier jedoch nicht immer als Lotterien bezeichnet. Häufig handelt es sich um Kaufentscheidungen, bei denen die alternativen Zustände unterschiedliche Aktien oder andere Güter darstellen, für deren fiktiven Erwerb sich die ProbandInnen entscheiden können bzw. zwischen denen sie wählen müssen. In der empirischen Umsetzung im Rahmen von Experimenten handelt es sich meist um Fälle, in denen die Akteure zwischen verschieden Lotterien wählen müssen. Wie wirtschaftswissenschaftliche Laborexperimente zeigen, führen Risikofreude und Risikoaversion der Akteure dazu, dass sie deutlich von der Orientierung an den Erwartungswerten abweichen. ${ }^{45}$

Lotterien sind ein standardisiertes Instrument zur Ermittlung des Risikoverhaltens in ökonomischen Laboren und zugleich wird der Mechanismus vielfältig eingesetzt, um die Präferenzen von ProbandInnen zu untersuchen. Welches Ergebnis beim Ausspielen einer Lotterie eintritt, soll nur vom Zufall abhängig sein.

\footnotetext{
${ }^{45}$ Das Bernoulli-Prinzip thematisiert diesen Aspekt der Entscheidungen unter Unsicherheit und verweist darauf, dass ein objektiver Vergleich des Erwartungsnutzens nicht genügt, sondern die Risikoeinstellungen bei der Ermittlung der individuellen Nutzenfunktion der Akteure von entscheidender Bedeutung sind. Risikoaverse Spieler werden durch mögliche negative Resultate der Lotterien, abgeschreckt " und wählen sicherere Alternativen, auch wenn diese einen deutlichen geringeren Erwartungsnutzen besitzen.
} 
In der Situation des Experiments soll ausgeschlossen werden, dass die ProbandInnen oder ExperimentatorInnen den Ausgang der Lotterie gezielt beeinflussen. Um dies auch im Labor zu veranschaulichen, werden bestimmte Verfahren eingesetzt. Häufig werden Münzen oder Würfel geworfen oder Karten gezogen, um die Zufallsziehung des Ergebnisses zu veranschaulichen und somit zugleich zu zeigen, dass dieses nicht durch die ExperimentatorInnen manipuliert wurde. In einer beobachteten Experimentserie zur Exklusivität von Wahlentscheidungen wurden die ProbandInnen beispielsweise mit diesem Lotterspiel konfrontiert:

„Als zusätzliche Belohnung für das Experiment geben wir Ihnen die Möglichkeit eine der Lotterien aus zu wählen. Am Ende des Experiments werden Sie einen 10-seitigen Würfel werfen. Wählen Sie eine der folgenden Lotterien:

Möglichkeit 1: Falls der Würfel 1,2 oder 3 zeigt, erhalten Sie 34 EUR, bei 4-10 nichts.

Möglichkeit 2: Falls der Würfel 1,2,3,4 oder 5 zeigt, erhalten Sie 20 EUR, bei 6-10 nichts.

Möglichkeit 3: Falls der Würfel 1,2,3,4,5,6 oder 7 zeigt, erhalten Sie 14 EUR, bei 8,9,10 nichts. “46

Am Ende des Experiments bekamen alle ProbandInnen nacheinander die zugesagte Möglichkeit, einen 10-seitigen Würfel zu werfen, um das Ergebnis der von ihnen gewählten Lotterie zu bestimmen. Dass die ProbandInnen die Höhe ihres erspielten Gewinns durch ihre eigenen Handlungen und Entscheidungen beeinflussen, wird für die ProbandInnen durch die Performativität des Würfelwurfs deutlich.

Die paradigmatische Orientierung am außenstrukturellen Merkmal der Randomisierung und die Demonstration der Zufallsabhängigkeit zeigt sich auch hier als ein zentrales Moment der Konzeption von Experimenten. Die Randomisierung bezieht sich hier jedoch nicht auf die zufällige Verteilung der ProbandInnen (und ihre personengebundenen Störgrößen) in den Kontroll- und Versuchsgruppen oder auf die Arbeitsplätze im Labor, sondern auf die eintretenden Ergebnisse. Die Glaubwürdigkeit des unbeeinflussten Ergebnisses ist von großer Bedeutung. Hier - wie auch oben im Zusammenhang mit dem Spielchip ausgeführt - werden gezielt Objekte eingesetzt, um die Unabhängigkeit des Ergebnisses vom Willen und Wollen der ExperimentatorInnen zu veranschaulichen. So gibt es für die

${ }^{46}$ Quelle: Bildschirminstruktionen ,Exklusivitäts-Experiment“. 
ProbandInnen keinen Grund zu vermuten, dass die angegebenen Wahrscheinlichkeiten nicht den Rahmenbedingungen im Experiment entsprechen würden. Bei diesen , vertrauensbildenden Maßnahmen' geht es um die Vermeidung von ,sozialen Störfaktoren“ im Sinne von Versuchsleiter-Effekten. Misstrauen gegenüber der Redlichkeit der Forschenden erhöht aus forscherischer Sicht stets die Gefahr, dass sich die ProbandInnen in ihrer situativen Rahmenbildung (frame-in-use) an ungeplanten Rahmungsaspekten orientieren. Diese könnten sich im Folgenden als unkontrollierter Störfaktor in den Daten niederschlagen. Die einzigen Informationen, die die ProbandInnen in einem Experiment für ihre Wahl zwischen verschiedenen Handlungsalternativen heranziehen sollen, sind der Ansicht der experimentellen Forschenden folgend, diejenigen, die durch die Darstellung der Spiel- und Entscheidungssituation gegeben sind. Am Beispiel der Lotterien wird ersichtlich, wie spezifische Mechanismen in der Hervorbringung des ökonomischen Laboratops eingesetzt werden.

Der Einsatz von Lotterien ist für die Forschenden mit den oben dargestellten konzeptionellen Annahmen verbunden. Lotterien bilden aus ihrer Sicht ein Verfahren zur Untersuchung des ökonomischen Entscheidungsverhaltens. Aus dieser Perspektive trägt die Nutzung von Lotterien zugleich zum Doing der spezifisch ökonomisch-rationalen Verhaltenserwartungen bei. Es wird dabei angenommen, dass das Sprachspiel der Forschenden dem der ProbandInnen angeglichen werden kann und sie das Konzept der Lotterien auf ähnliche Weise verstehen.

Lotterien tragen in typischen Verbindung einerseits zum Doing einer spezifischen Experimentsituation und zugleich zum Doing der regelkonformen Verhaltenserwartungen bei. Die paradigmatische Orientierung am außenstrukturellen Merkmal der Randomisierung und die Demonstration der Zufallsabhängigkeit führt auch hier wieder zur gleichzeitigen Verankerung von Spiel- und Experimentmodul. Teil der spezifischen Spielrahmung des Laborexperiments und zugleich auch des Experimenthaften ist es, den Zufall und die Konsequenzen, die sich aus seinem Wirken ergeben, zu akzeptieren. Zugleich wird auch eine typische Verankerung von Spiel- und Ökonomiemodul ersichtlich. Der Spielrahmen, welcher von den Forschenden präsentiert wird, ist auf strategisches Handeln und bewusstes Entscheiden ausgerichtet. Den ProbandInnen werden Informationen präsentiert, die es ihnen erlauben sollen, zwischen den Wahlalternativen abzuwägen und dies auch zu tun. Zentrales Kriterium ist dabei der in Aussicht gestellte Gewinn. 


\subsubsection{Die Raumstruktur und die materielle Ausstattung des Laboratops}

Während die Gliederungsmerkmale des Laborexperiments die zeitliche Klammer der Modulation darstellten, wird die räumliche Klammer durch den Laborraum selbst ausgebildet. Die Ausgestaltung der spezifischen Laborräume, in denen die Experimente durchgeführt werden, stellt ein Merkmal der Binnenstruktur des untersuchten Kommunikationsprozesses der Rahmenbildung dar. Die raumstrukturellen Merkmale überdauern die einzelnen Interaktionssituationen und sind von diesen unabhängig. In ihrer Bedeutung und rahmenwirksamen Funktion für das Phänomen des Laborexperiments ist die räumlich-materielle Ausgestaltung der Laborräume stark von paradigmatischen Ansprüchen der Forschenden geprägt, welche auf der Ebene der Außenstruktur dargestellt wurden. Einerseits gilt es hier, den Standards der experimentellen Methodologie zu entsprechen (Abschnitt 2.1), und andererseits ein Erhebungsumfeld zu schaffen, welches den disziplinspezifischen Modellannahmen an Akteure und ihr Entscheidungsverhalten (Abschnitt 5.2.1) Rechnung trägt. Zentrale Elemente des Settings stellen dabei Objektivationen kommunikativen Handels dar, die sich im Rahmenbildungsprozesses des Laboratops als wirkmächtig erweisen. Im Folgenden wird nun betrachtet, wie das Setting des Labors gestaltet wird, um den forschungsleitenden Ansprüchen der Forschenden zu entsprechen, und wie Elemente des räumlich-materiellen Settings die kommunikative Konstruktion des ökonomischen Laboratops auf den unterschiedlichen Ebenen des Doings unterstützen. Zu einem besseren Verständnis des Nachfolgenden werden die wichtigen Elemente des Laborraums in einem ersten Schritt noch einmal beschrieben und durch Abbildung 5.11 illustriert.

Die Wände des Raums sind weiß gestrichen, sein Fußboden ist blau. An der unteren Wand des Raums befindet sich eine Tür, an der eine braune Kiste befestigt ist. An der gegenüberliegenden Seite des Raums ist eine große Fensterfront zu sehen. Durch die Nutzung grauer Trennwände wurde eine weitere Unterteilung des Raums realisiert. Spezifischer wurden 30 graue Trennwände aufgestellt und damit 25 einzelne Kabinen geschaffen. Es handelt sich bei diesen Kabinen um die Arbeitsplätze für die VersuchsteilnehmerInnen. Diese sind jeweils von drei Seiten geschlossen und können somit nur von einer Seite aus betreten werden. An der linken sowie an der rechten Wand des Raumes befinden sich jeweils sieben Kabinen und an der unteren Seite des Raumes, rechts neben der Tür, drei weitere Arbeitsplätze. In der Mitte des Raumes sind in einer Zweierreihe acht weitere Arbeitsplätze eingerichtet. Zwischen den an der Wand positionierten Arbeitsplätzen und jenen in der Mitte verläuft ein Gang. Die einzelnen Kabinen 


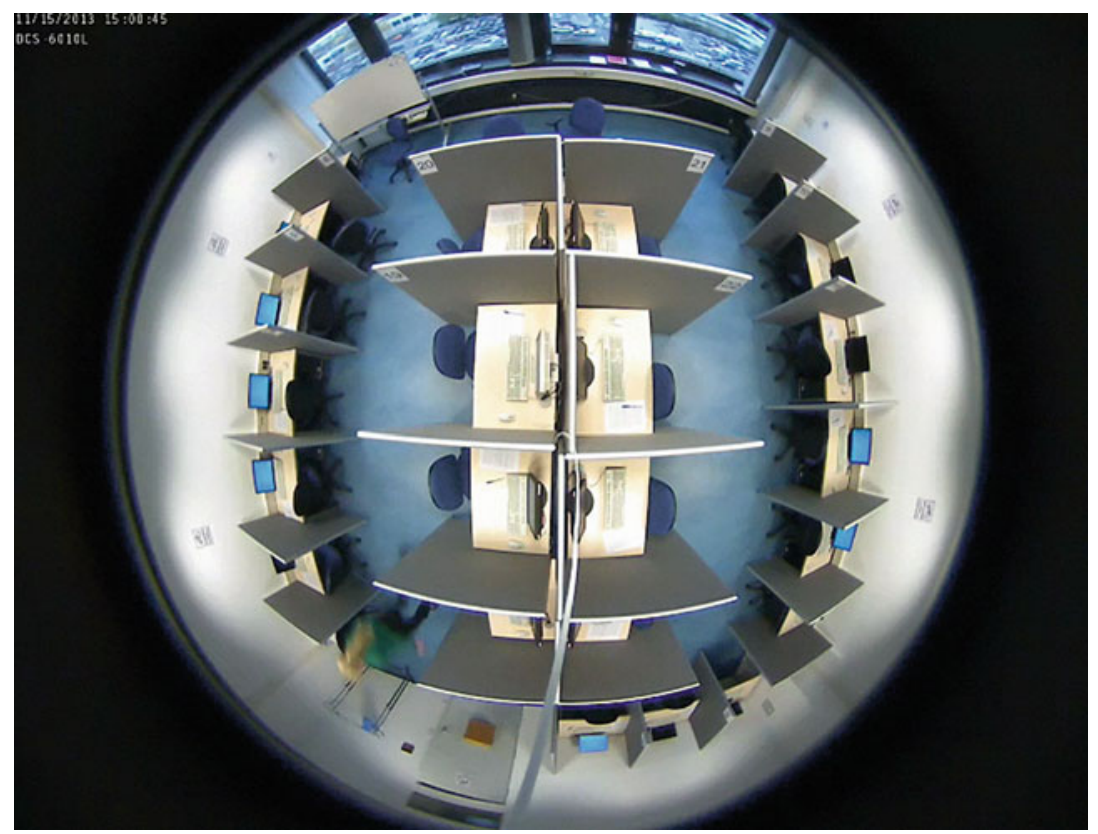

Abb.5.11 Raumstruktur des Labors

sind mit nummerierten Schildern von 1 bis 25 versehen. An der linken Wandseite des Raums befinden sich die Kabinen mit den Nummer 1 bis 7, auf der gegenüberliegenden rechten Wandseite diejenigen mit den Nummern 8 bis 14. Im mittleren Achterblock sind links die Kabinen 17 bis 20 und rechts diejenigen mit der Nummer 21 bis 24 angeordnet. An der unteren Wandseite, rechts neben der Tür, sind die Kabinen 15, 16 und 25 positioniert. Die Beschriftung mit Arbeitsplatznummern ist dabei stets so angebracht, dass sie aus der Laufrichtung von der Eingangstür zur Fensterfront eingesehen werden kann.

Die 25 Kabinen sind jeweils gleichartig ausgestattet (Abb. 5.12a und 5.12b). Sie verfügen über einen Tisch mit einer hellen Arbeitsplatte, der in allen Kabinen jeweils die gleiche Größe besitzt, sowie einen blauen Drehstuhl und einen Desktopcomputer. Auf der Tischplatte befinden sich in allen Kabinen ein Computerbildschirm, eine Tastatur und eine Computermaus. Zur weiteren Ausstattung der Laborarbeitsplätze gehören vor Beginn eines Experiments ein Stift und einige Seiten bedrucktes Papier. Einen weiteren Aspekt der Laborausstattung stellt ein 
mobiles Whiteboard mit einem rollbaren Gestell dar, welches sich in der linken oberen Ecke des Raumes befindet. An der oberen Seite des mittigen Achterblocks von Kabinen, nahe der Fensterfront, befindet sich ein weiterer Sitzbereich mit zwei Drehstühlen und zwei Computermonitoren und Desktop-Computern.

Den Aussagen der ökonomischen ForscherInnen folgend, wird das materielle Umfeld des Labors von ihnen als neutraler Raum verstanden. Den Forschenden ist durchaus bewusst, dass das Experimentallabor ein sehr spezielles und artifizielles Setting ist. Die Bezeichnung, neutral ' verweist hier auf den Anspruch des Konstanthaltens der Randbedingungen für die Erhebungssituation. Der Laborraum soll von allen ProbandInnen auf die gleiche Art und Weise verstanden werden und für alle die gleiche räumliche Entscheidungsbasis bieten. Bei der Durchführung der Experimente wird großer Wert auf die Gleichartigkeit der Ausstattung von mobilen ${ }^{47}$ und immobilen Elementen der einzelnen Arbeitsplätze gelegt.

Abb. 5.12a

Arbeitskabinen (Totale)

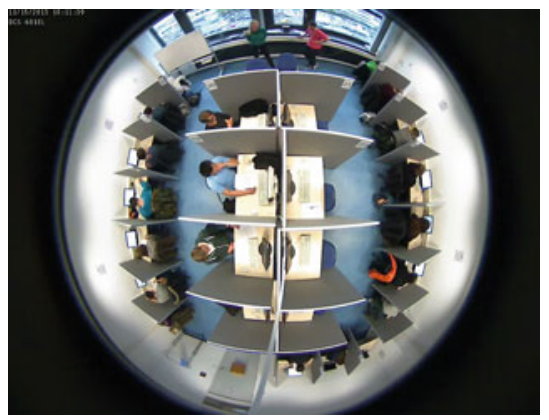

Das Labor besitzt eine spezifische Interaktionsarchitektur (Hausendorf \& Schmitt 2013), die die Möglichkeiten des interaktiven Austauschs zwischen den Handelnden beeinflusst und begrenzt. Wie die deskriptive Darstellung des Settings ausweist, sind bestimmte Bereiche im Labor zum Sitzen und andere zum Laufen vorgesehen. Durch die angebrachten Schilder an den Trennwänden wird auch aufgezeigt, dass es eine übliche Laufrichtung gibt, in der sich die ProbandInnen im Labor bewegen. Die nummerierten Schilder sind von der Tür zur Fensterseite einsichtig, jedoch nicht ohne weiteres anders herum. Der Gang zwischen den

\footnotetext{
${ }^{47}$ Mobile Elemente des räumlich-materiellen Settings meint hier all jene Objekte, die beweglich sind und vor Beginn der Experimente in geordneter und gleichartiger Weise an den Arbeitsplätzen arrangiert werden. Dies umfasst die Blätter mit den Instruktionen, den Stift, die Tastatur und die Computermaus sowie den Drehstuhl, mit welchem jeder Arbeitsplatz ausgestattet ist.
} 
Abb. 5.12b

Arbeitskabinen (Zoom)

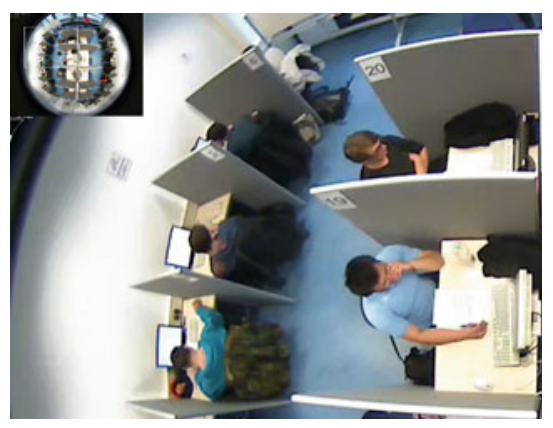

Arbeitsplätzen ermöglicht es den ProbandInnen einerseits, ihre Arbeitsplätze zu erreichen. Zugleich erlaubt er auch den ExperimentatorInnen, an die einzelnen Arbeitsplätze heranzutreten oder im Vorbeigehen einen Blick auf den Bildschirm der einzelnen ProbandInnen zu werfen. ${ }^{48}$

Mittels der Trennwände werden die Arbeitsplätze der ProbandInnen voneinander separiert. Sie stellen eine visuelle Barriere zwischen den TeilnehmerInnen dar. In jeder Kabine kann nur eine Person auf dem dort vorgesehenen Drehstuhl Platz nehmen. Richten die Teilnehmenden ihre Aufmerksamkeit auf den Computerbildschirm, so befinden sich die Arbeitsplätze der angrenzenden Reihe hinter ihrem Rücken. Wenn die ProbandInnen in Armreichweite zu ihren Eingabegeräten (Tastatur und Maus) sitzen, können sie nur die sie umgebenden Kabinenwände sehen. Die anderen Handelnden sind somit außerhalb ihres Blickfeldes. Nur wenn sich die ProbandInnen mit ihrem Drehstuhl aktiv aus der Kabine bewegen, können sie den Gang entlangschauen und in die Arbeitsplätze anderer Versuchspersonen blicken. Von ihren Arbeitsplätzen aus haben sie keine Einsicht auf den Arbeitsplatz der ExperimentatorInnen.

Der Laborraum ist durch eine spezifische Raumstruktur charakterisiert. Der Begriff der „Interaktionsarchitektur“ (Hausendorf \& Schmitt 2013) scheint passend, um den performativen Einfluss des räumlich-materiellen Settings auf die Konstruktions- und Rahmenbildungsprozesse des Laboratops zu adressieren. Einerseits befinden sich alle Handelnden in einer gemeinsamen sozialen Situation im Laborraum. Andererseits werden durch die Trennwände weitere kleine

\footnotetext{
${ }^{48}$ Dieser Aspekt wird im Analyseteil der situativen Realisierung noch von Belang sein. Ein typisches Merkmal der interaktiven Handlungskoordination stellt die ,Technik des Rundgangs“ (Abschnitt 5.4.6) der ExperimentatorInnen dar, bei welchem diese den visuellen Zugriff gezielt nutzen, um den Arbeitsstand der ProbandInnen zu überprüfen.
} 
Räume geschaffen, die zur exklusiven Kommunikation zwischen den ExperimentatorInnen und einzelnen ProbandInnen dienen und die Versuchspersonen visuell und begrenzt auch akustisch voneinander abschirmen. ${ }^{49}$ Goffmans grundlegender Definition sozialer Situationen folgend, bezeichnet eine soziale Situation ,any physical arena anywhere within which two or more individuals find themselves in visual and aural range of one another" (Goffman 1972: 63). Soziale Situationen zeichnen sich durch räumliche und zeitliche Kopräsenz aus sowie durch ,response presence" (Godwin 1981). Letztere meint, dass mindestens zwei Akteure ihre Handlungen wechselseitig koordinieren, wobei dies nicht symmetrisch erfolgen muss. Auch nicht Handeln oder das Erdulden von Handlungsbeschränkungen entspricht diesem Aspekt der Koordination. Meyer et al. (2016) verweisen darauf, dass die Form der Kopräsenz ${ }^{50}$ ein zentrales Definitionskriterium der Spezifik von Interaktionssituationen bildet. Teil der Rahmenbildung in der Laborsituation ist ein charakteristisches Wechselspiel von Arten der Kopräsenz und räumlich-materiellen Aspekten der Interaktionssituation.

Durch das Setting werden einerseits eine körperliche Kopräsenz der ProbandInnen und ExperimentatorInnen und zugleich ihre visuelle Separierung von- und untereinander ermöglicht. Es erfolgt eine räumliche Vereinzelung der ProbandInnen in den einzelnen Kabinen. Die materielle Struktur des Laborraums trägt hier entscheidenden Anteil an der Induzierung charakteristischer Rahmungselemente. Der für den Prozess des Laborexperiments charakteristische Wechsel zwischen zentrierten, nicht-zentrierten und multi-zentrierten Interaktionssituationen wird maßgeblich durch die materielle Struktur des Laborraums ermöglicht. Die Platzsuche der ProbandInnen, die Momente der gemeinsamen Ansprache, des individuellen Fragenstellens oder der vereinzelten Bearbeitung von Aufgabenstellungen lassen sich hinsichtlich ihrer charakteristischen Momente als jeweils andere Formen der Versammlung ${ }^{51}$ im Sinne Goffmans spezifizieren. Sie alle bilden typische

\footnotetext{
${ }^{49}$ In mehreren Interviews verwiesen ProbandInnen darauf, dass sie durch Tippgeräusche akustisch sehr wohl bestimmen könnten, welche der benachbarten ProbandInnen welche Rollen im Experiment einnehmen. Der Grund liegt primär in der Sequenzialität der Experimente, um die auch die ProbandInnen wissen. In vielen Experimenten sind die Handlungsaufforderungen, mit denen die ProbandInnen durch die Bildschirmoberflächen konfrontiert werden, nicht für alle Teilnehmenden gleichartig. Sie unterschieden sich vielmehr je nach zugewiesener Rolle. Aufmerksame ProbandInnen können somit auf akustischem Weg Kenntnis davon erhalten, ob den ProbandInnen in anliegenden Kabinen die gleiche Rolle zugewiesen wurde wie ihnen. ${ }^{50}$ Wie Abschnitt 5.4.6 aufzeigt, wird die Herstellung spezifischer Formen von Kopräsenz als Ethnomethode der Interaktionssteuerung im Labor eingesetzt.

${ }^{51}$ Bezug genommen wird hier auf die Goffmansche Differenzierung unterschiedlicher Arten von Interaktionssituationen in Kopräsenz, die er als Versammlungen von Interaktionspartnern bezeichnet (siehe dazu Abschnitt 3.1.1).
} 
Momente des Laborgeschehens und werden maßgeblich durch die räumliche Struktur des Labors geprägt. So zielt die Datenerhebung im Labor beispielsweise gerade darauf $\mathrm{ab}$, eine multi-zentrierte Interaktionssituation $\mathrm{zu}$ schaffen. In dieser Situation sind die ProbandInnen zwar räumlich nah, doch zugleich handelt es sich um eine Versammlung mit ,vielen Brennpunkten“ (Goffman 1973: 21). Jede ProbandIn muss die datenerhebungsrelevanten Entscheidungen für sich allein treffen. Die Vereinzelung in den Arbeitskabinen und die technische Vermittlung von Handlungsaufforderungen oder Entscheidungssituationen sind hier zentrale Elemente, die diese Situation hervorbringen.

Im ökonomischen Laboratop bilden die Trennwände Objektivationen kommunikativen Handelns. Ihre Wirkmächtigkeit wird im Vollzug des Laborgeschehens deutlich. Ganz im Sinne einer ,Thingification“ (Barad 2013) stehen die Trennwände als materielle Objekte für kommunikatives Handeln, welches in seiner Wirkung und Bedeutung für die Handelnden verstetigt ist. Die Trennwände können als Objektivationen der Informationskontrolle betrachtet werden. In besonderem Maße begünstigen sie die Umsetzung des Kommunikationsverbots und der Anonymisierung im Labor. Die Trennwände bilden hier eine bleibende materielle Instanz, die die Umsetzung der Anforderungen der ForscherInnen bestärkt. Der verbal und schriftlich explizierten Regel des Kommunikationsverbots zwischen den ProbandInnen wird hier auf materielle Weise Ausdruck verliehen. Es darf nicht zu benachbarten Versuchsteilnehmenden geschaut werden, um an handlungsrelevante Informationen zu gelangen oder sich durch Face-to-Face Kommunikation auszutauschen. Die Kontrolle, dass keine visuelle Kontaktaufnahme und kein Austausch zwischen den ProbandInnen erfolgt, muss dabei nicht mehr allein durch die Mitglieder des Laborteams erfolgen. Die Trennwände bewirken als gleichartige und damit standardisierte Objektivationen, dass diese Kontrolle realisiert wird. In diesem Sinne bilden sie eine standardisierte Kontrollinstanz des Kommunikationsverbots. Als Objektivation kommunikativen Handels besitzen die Trennwände einen spezifischen performativen Charakter. Dieser ist durch die Permanenz der Objekte und den Eigensinn der Materialität der Trennwände begründet. Indem sie als materielle Instanzen zur Durchsetzung und Aufrechterhaltung der von den Forschenden kommunizierten Regeln beitragen, bilden die Trennwände eine materielle Verankerung des Spielmoduls. Ein Zuwiderhandeln durch die ProbandInnen ist durchaus möglich, indem sie mit ihrem Drehstuhl aktiv nach hinten rücken und ihren Sichtbereich so vergrößern. In diesen Fall obliegt die situative Kontrolle (beziehungsweise Intervention) gegenüber diesem Vorgehen den ExperimentatorInnen, welche die Durchführung der Experimente überwachen und mittels typischer situativer Methoden der Handlungskoordinierung das regelkonforme Handeln der Teilnehmenden überprüfen und situativ angemessen nachjustieren. 
Durch die Trennwände wird zugleich ein visuell geschützter Raum für die Handlungen einzelner ProbandInnen im Experiment geschaffen. Es wird auf diese Weise der Verdacht unterbunden, dass benachbarte TeilnehmerInnen auf den eigenen Arbeitsplatz schauen könnten, um auf diesem Wege Informationen über die eigenen Handlungen zu erhalten. Dieser Aspekt ist für die Durchführung von Experimenten von großem Belang und zielt auf die materielle Umsetzung der durch die Forschenden zugesagten Anonymität der Rollen und Handlungen der Versuchspersonen ab. Die Umsetzung der Anonymisierung wird durch das Zusammenspiel mehrerer Ethnomethoden und typischer materieller Objektivationen erzeugt. Die Trennwände bilden dabei Objektivationen der visuellen Abschirmung gegenüber den anderen ProbandInnen. Sie verleihen der schriftlich und verbal zugesagten Anonymität durch die Forschenden einen materiellen Ausdruck. Die materielle Objektivation der Anonymität durch die Trennwände wird zudem durch weitere Ethnomethoden ergänzt.

Die oben dargestellte randomisierte Sitzplatzzuordnung, die durch die Trennwände realisierte Vereinzelung und Abschirmung der ProbandInnen in ihren Kabinen und das Unterbinden von Face-to-Face Interaktionen bestärken die Annahme der ProbandInnen, dass im Vollzug des Experiments die Anonymität ihrer Rolle und Entscheidungen gewahrt bleibt. Die Anonymisierung der ProbandInnen führt zu einem Herunterkühlen des sozialen Settings. Amoralisches oder egoistisches Handeln kann in Situationen deutlich leichter praktiziert werden, in denen Handlungen nicht persönlich zugeordnet werden können und die Handelnden keine persönlichen Sanktionen der anderen ProbandInnen zu fürchten haben. VersuchsteilnehmerInnen berichteten in den Interviews, dass es in einigen Spielen, wie beispielsweise im Fall des später dargestellten Mindestlohn-Experiments, die beste Strategie gewesen sei, ,fies zu spielen“, um die eigene Auszahlung $\mathrm{zu}$ erhöhen und einen möglichst hohen Gewinn zu erzielen. Dass dieses Verhalten moralisch zweifelhaft war, räumten die Interviewten selbst ein, doch da ihre Handlungen nicht auf sie persönlich zurückzuführen waren, zielten sie nur darauf ab, die bestmögliche Strategie zur Maximierung ihres Gewinns zu nutzen. Das Verfahren der Anonymisierung begünstigt somit nicht nur die Minimierung des Einflusses von sozialen Erwünschtheitseffekten, sondern befördert zugleich eine Rahmung der Experimentsituation, die sich an ökonomisch-rationalen und nutzenmaximierenden Aspekten orientieren kann. Dies bedeutet nicht, dass alle ProbandInnen aufgrund der Anonymisierung amoralisch handeln würden, doch die Abschirmung der ProbandInnen voneinander und die gezielte Beschränkung der Kommunikation eröffnen Handlungsspielräume und -möglichkeiten, die in einer sozialen Face-to-Face Interaktion mit hoher Wahrscheinlichkeit deutlich stärker sanktioniert werden würden. 
Zusammenfassend lässt sich festhalten, dass das ökonomische Laboratop auch in räumlich-materieller Hinsicht darauf ausgerichtet ist, ein kontrolliertes Handlungsumfeld für die Datenerhebung zu bieten. Eine Kontrolle über die Informationen, die den ProbandInnen zur Verfügung stehen, ist aus Sicht der Forschenden eine maßgebliche Voraussetzung für die Umsetzung der Modellannahmen, denen sie folgen. Die gleichartige materielle Gestaltung und Ausstattung der ProbandInnenarbeitsplätze unterstützt das Doing einer spezifischen Laborsituation. Bezug genommen wird hier auf das Konstanthalten der Randbedingungen und die Standardisierung der Erhebungssituation. In Kombination mit der SUVTA-Annahme erlaubt es, von einer hohen internen Validität der Ergebnisse der Datenerhebung im Labor auszugehen. Am Beispiel der Objektivation der Trennwände wurde zudem aufgezeigt, wie charakteristische Elemente der Laborausstattung das Doing der rationalen und regelkonformen Verhaltenserwartungen unterstützen. Das Zuwiderhandeln der ProbandInnen kann im dargestellten Beispiel des Kommunikationsverbots dabei nicht ausgeschlossen werden. Der Handlungsspielraum der ProbandInnen, welcher keiner direkten Kontrolle der Labormitglieder zugänglich ist, wird begrenzt und deviantes Handeln auf diese Weise deutlich erschwert. Darüber hinaus werden die materiellen Objektivationen des Kommunikationsverbots durch weitere Ethnomethoden der Handlungskoordinierung ergänzt, die auf der Analyseebene der situativen Realisierung thematisiert werden. Wie dargestellt wurde, bilden die Trennwände auch eine materielle Voraussetzung für die Umsetzung der Anonymisierung im Labor. Sie sind als Objektivationen der Vereinzelung und Anonymisierung der ProbandInnen zu betrachten und leisten dabei einen Beitrag zum Doing ökonomisch-rationaler Verhaltenserwartungen.

Das räumlich-materielle Setting und die Unterteilung in die einzelnen Arbeitskabinen trägt somit sowohl zum Doings einer spezifischen Laborsituation als auch zum Doing der rationalen und regelkonformen Verhaltenserwartungen bei. Das Konstanthalten der Versuchsbedingungen über mehrere Sessions einer Treatmentvariation hinweg ist eine maßgebliche Voraussetzung, um die Daten der einzelnen Sessions später zusammenfassen zu können und sie mit anderen Variationen der Versuchsanordnung zu vergleichen. Durch die Standardisierung der Arbeitskabinen hinsichtlich ihrer räumlich-dingliche Ausgestaltung werden die Anforderungen an ein gleichartiges Entscheidungsumfeld umgesetzt. Sie tragen somit auf materieller Ebene zu Verankerung des Experimentmoduls bei. Die Raumstruktur des Labors unterstützt zudem die von den Forschenden angestrebte regelkonforme Art der Interaktion. In den unterschiedlichen Phasen des Handlungsgeschehens im Labor werden je spezifische Ethnomethoden der Interaktionssteuerung zwischen ExperimentatorInnen und ProbandInnen eingesetzt. Sie dienen primär der Informationskontrolle oder der Unterstützung der geplanten 
Durchführung des Experimentablaufs und werden auf der Ebene der situativen Realisierung genauer betrachtet. ${ }^{52}$ Die Raumstruktur trägt somit zugleich zur Verankerung und Durchsetzung des Spielmoduls bei, indem sie die materielle Grundlage für verschiedenartige Interaktionsräume bildet.

\subsubsection{Mediation der kommunikativen Handlungen}

Die räumlich-materielle Gestaltung des Laborsettings unterstützt die Kontrolle und Koordinierung der ablaufenden Interaktionen. Wie oben dargestellt, wurden durch den Einsatz der Trennwände innerhalb des Labors kleine Arbeitsräume für die Versuchsteilnehmenden geschaffen. Durch die Eingabegeräte der Computer ist die Positionierung der ProbandInnen innerhalb der Arbeitskabinen in gewissem Maße determiniert. Um die Eingabegeräte zu erreichen, müssen die ProbandInnen so weit in die Kabine hineinrücken, dass sie visuell von den benachbarten Versuchsteilnehmenden abgeschirmt sind. Die nachfolgenden Ausführungen widmen sich der typischen Form der technisch vermittelten Mediation kommunikativer Handlungen im Labor. Diese ist ein weiteres wichtiges Merkmal der Rahmenbildung des ökonomischen Laboratops und verankert hier Experiment-, Spielund Ökonomiemodul in technisch-materieller Weise. ${ }^{53}$ Laborexperimente sind durch einen charakteristischen Einsatz von Technik gekennzeichnet und beinhalten stets gewisse Abschnitte, in denen die ProbandInnen an den Computern bestimmte Aufgaben erfüllen müssen. Diese Situationen werden den ProbandInnen mittels des Computerbildschirms präsentiert. Mit Maus und Tastatur machen die Teilnehmenden Eingaben in die vorgegebenen Felder auf dem Bildschirm oder führen andere Aktionen aus, zu denen sie aufgefordert werden. Die am Computer generierten Daten bilden die Grundlage für die wissenschaftlichen Analysen und Auswertungen der Forschenden.

\footnotetext{
${ }^{52}$ Beispiele bilden hier die Ausführungen zum „Fragen stellen und beantworten“ (Abschnitt 5.4.3) oder der „Rundgang der ExperimentatorInnen“ (Abschnitt 5.4.6).

${ }^{53}$ In zeitlicher Hinsicht erfolgt die Koordination des Ablaufs hierbei durch ein Zusammenspiel des direkten kommunikativen Handelns der ExperimentatorInnen und den technisch vermittelten kommunikativen Handlungen, welche durch die Informationen und Handlungsaufforderungen an den Computerbildschirmen präsentiert und objektiviert werden. Die Abschnitt 5.4.4 und 5.4.5 widmen sich diesen Aspekten der Rahmenbildung.
} 
Auch im Fall von zeichenhaften Objektivationen spielt ihre spezifische Materialität eine entscheidende Rolle, denn die Modalitäten ${ }^{54}$ bieten verschiedene Optionen der Sinngebung. Das Zusammenspiel verschiedener Modalitäten wird nach Kress (2010) als Multimodalität bezeichnet. Im Folgenden wird genauer betrachtet, welche technischen Mittel eingesetzt werden und wie sie die kommunikativen Inhalte verändern und prägen. Für die vorliegende Analyse sind die Wirkzusammenhänge oder „Vermittlungsbeziehungen“ (Rammert 2007: 55) zwischen den typischen Formen kommunikativen Handels und den aus ihnen hervorgehenden Objektivationen von besonderem Interesse. Mediation bezeichnet die technische Vermittlung von kommunikativen Inhalten. Denn die kommunikative Konstruktion der Wirklichkeit lässt sich mit unterschiedlichen Medien unterschiedlichen gestalten. Für die Forschungsfrage nach dem Prozess der Rahmenbildung und -verankerung, der das ökonomische Laboratop in seiner Eigengesetzlichkeit hervortreten lässt, ist Hepps Begriff der „Prägkraft“ (Hepp 2013: 104, 2011: 55 ff.) nützlich. Dieser lenkt den Blick auf die Spezifik eines Mediums im Prozess der Kommunikation und somit auf den prägenden Einfluss der Performativität, welche dieses Medium im Handlungsvollzug ausübt. Der Einsatz von Medien in der kommunikativen Konstruktion der Laborsituation macht sich diese prägende Wirkung gezielt zu Nutze.

Die im wirtschaftswissenschaftlichen Experimentallabor eingesetzten Softwareprogramme unterstützen in technisch-standardisierter Weise die Koordinierung der Handlungsphasen. Durch den Einsatz der Softwareprogramme wird auf standardisierte Weise eine Sequenzialität und punktuelle Synchronisation des , Workflows ' der ProbandInnen geschaffen ${ }^{55}$ Auch hier wird wieder eine größtmögliche Standarisierung zwischen den einzelnen Treatmentvariationen angestrebt. Die Programmierung soll sich nur hinsichtlich der Aspekte unterscheiden, die der gezielten Manipulation des Einflussfaktors und somit der zu testenden Hypothese, entsprechen. In den von mir beobachteten Experimenten, wurde das Softwareprogramm $z$-Tree genutzt, das eigens für die Durchführung computergestützter Laborexperimente konzipiert wurde (Fischbacher 2007). Die Computer im Labor sind durch ein Netzwerk miteinander verbunden. Während auf dem Computer

\footnotetext{
${ }^{54}$ Das Verhältnis zwischen der Körperlichkeit, Sinnlichkeit und Materialität der Objektivation wird als Modalität bezeichnet und nimmt Bezug auf die Verknüpfung zwischen den menschlichen Sinnen und den Objektivierungen.

${ }^{55}$ Der Begriff ,Prägkraft“" sollte nicht im technikdeterministischen Sinne missverstanden werden, sondern steht hier in der sozialkonstruktivistischen Tradition und wird in diesem Sinne verwendet. Er verweist auf das Spannungsverhältnis von Institutionalisierung und Verdinglichung (Hepp 2011: 55 ff.), welches sich in der Performativität materieller Objektivationen niederschlägt.
} 
der ExperimentatorInnen das Programm z-Tree installiert ist, verfügt jeder Computer an den Arbeitsplätzen der ProbandInnen über die Nutzer-Software z-Leaf. Anhand von Screenshots der Applikationen von z-Leaf wird nun illustriert, wie die typische sequenzielle Organisation der Abläufe und die Standardisierung der Kommunikation durch die eingesetzte Software und die ExperimentatorInnen im Labor realisiert wird.

Bereits vor der Durchführung der Datenerhebung im Labor wird im Zuge der Programmierung in z-Tree ein Ablaufschema einzelner Sequenzen festgelegt, die die ProbandInnen im Zuge der Datenerhebung im Labor durchlaufen sollen. Mittels des Programms z-Tree werden verschiedener Bildschirmoberflächen für die einzelnen Treatmentvariationen produziert, die später an den Computern der ProbandInnen über das Programm z-Leaf ausgegeben werden. Der textliche Inhalt, die Grafiken und Schaltflächen, mit denen die ProbandInnen interagieren sollen, werden definiert und durch technische Mitarbeiter des Labors oder geschulte Forschende selbst mittels des Programms zusammengestellt.

Wie bereits mehrfach erwähnt, werden ProbandInnen in einem Experiment häufig unterschiedliche Rollen zugewiesen. Die Aktions- und Handlungsspielräume der ProbandInnen unterscheiden sich dabei in Abhängigkeit von ihren Rollen. In der Programmierung werden die Parameter festgelegt, nach denen bestimmte Handlungen der ProbandInnen zu bestimmten Resultaten in den Experimenten führen. ${ }^{56}$ Bilden mehrere ProbandInnen im Rahmen eines Experiments Spielpartner oder -gruppen, so wird im Zuge der Programmierung festgelegt, wie die Zuordnung der ProbandInnen zu diesen Gruppen erfolgen soll. In einigen Experimenten ist es vorgesehen, dass die zu Beginn zugeteilten Rollen der ProbandInnen und die Spielergruppen über das ganze Experiment Bestand haben. In anderen Experimenten werden den Teilnehmenden zu Beginn Rollen zugeordnet, doch die Zusammensetzung der Spielergruppe wird zufällig bestimmt und ändert sich von Runde zu Runde. Die sequenziellen Abfolgen der Handlungen der Gruppenmitglieder, ihrer Aktionen und die Möglichkeiten ihrer Interaktion und Kommunikation werden dabei durch die ExperimentatorInnen in der Programmierung vordefiniert. 57

Vom Arbeitsplatz der ExperimentatorInnen aus werden die für das Experiment benötigten Programme gestartet. Mit Hilfe eines Übersichtsbildschirms können

\footnotetext{
${ }^{56}$ An dieser Stelle sei daran erinnert, dass wirtschaftswissenschaftliche Experimente explizit darauf verweisen, dass die Entlohnung entscheidungsabhängig erfolgt und nicht vorher festgelegt ist.

${ }^{57}$ Im Abschnitt 5.4.5 der situativen Realisierungsebene wird anhand eines Beispiels aufgezeigt, wie der technisch vermittelte sequenzielle Ablauf einer Spielergruppe umgesetzt wird und wie er sich an den Bildschirmen der ProbandInnen darstellt.
} 
die ExperimentatorInnen zudem die ablaufenden Prozesse im Labor überwachen und den Arbeitsfortschritt an den einzelnen Arbeitsplätzen im Labor kontrollieren. Die beiden nachfolgenden Abbildungen (Abb. 5.13 und Abb. 5.14) stellen Screenshots eben dieser Übersichtsapplikationen dar. Während Abbildung 5.13, die „Clients' Table“, Auskunft über die spezifische Handlungsphase gibt, mit welcher ProbandInnen an den einzelnen Arbeitsplätzen gerade befasst sind, erfasst und visualisiert Abbildung 5.14 die „Subjects' Table“, die Handlungsentscheidungen und weitere experimentspezifische Variablen für jede einzelne Versuchsperson, die am durchgeführten Experiment teilnimmt. Die oben dargestellte Anonymisierung der Rollen und Handlungen der ProbandInnen gilt nur für die ProbandInnen untereinander, aber nicht gleichsam gegenüber den Forschenden. Durch das Ziehen des Spielchips erfolgt auch gegenüber den ExperimentatorInnen eine Entkopplung der personenbezogenen Daten von den individuellen Personen. Im Verlauf des Experiments ist es jedoch für die geschulten Labormitglieder recht einfach, mittels des Übersichtsbildschirms zu bestimmen, welche ProbandInnen welche Rolle im Experiment einnimmt. Auf die Relevanz des Übersichtsbildschirms und seine Verknüpfung mit gezielt eingesetzten Methoden der situativen Handlungskoordination wird im folgenden Analyseabschnitt zur situativen Realisierung noch detaillierter eingegangen.

Von grundlegender Bedeutung für die Rahmung der Laborsituation ist, dass die Programmierung der Experimente einen sequenz- bzw. rundenbasierten Ablauf vorsieht. Im Ablauf der Datenerhebung im Labor werden die einzelnen Sequenzen Schritt für Schritt abgearbeitet. Erst wenn alle ProbandInnen im Labor einen Handlungsabschnitt abgeschlossen haben, kann zur nächsten Handlungssequenz übergegangen werden. Dadurch ergeben sich Wartezeiten für die ProbandInnen. Als Konsequenz daraus zeigt sich in den Experimenten ein typischer Wechsel zwischen einer Reihe von aktiven Bildschirmen und einem Bildschirm, der die ProbandInnen zum Warten auffordert (Abb. 5.15). Dieser besitzt keine Schaltfläche und eröffnet den ProbandInnen auch keine technisch vermittelte Handlungsoption.

Bei den aktiven Bildschirmen lassen sich wiederum typische Formen unterscheiden. Es gibt Bildschirme, in denen ProbandInnen durch das Klicken eines Buttons bestätigen müssen, dass sie gewisse Informationen zur Kenntnis genommen haben (Abb. 5.16). Und es gibt solche, in denen die Teilnehmenden Entscheidungen treffen müssen. In den folgenden zwei Abbildungen müssen sie beispielsweise bestimmte Zahlenwerte eintragen (Abb. 5.17) und durch Anklicken eine von mehreren Optionen auswählen (Abb. 5.18). 


\begin{tabular}{|c|c|c|c|}
\hline [3] Clients' Table & & & 00 \\
\hline 16 clients & state & time & \\
\hline 1 & $\cdots$ Ofter period < max_period $\cdots$ & .26 & \\
\hline 2 & $\cdots$ offer period < max_period $\cdots$ & -26 & \\
\hline 3 & $\cdots$ offer period < max_period $\cdots$ & .26 & \\
\hline 4 & $\cdots$ Offer period < max_period $\cdots$ & .26 & \\
\hline 5 & $\cdots$ Offer period < max_period $\cdots$ & .26 & \\
\hline 6 & $\cdots$ Offer period < max_period $\cdots$ & .26 & \\
\hline 7 & $\cdots$ offer period < max_period $\cdots$ & -26 & \\
\hline 8 & $\cdots$ Offer period < max_period $\cdots$ & -26 & \\
\hline 9 & $\cdots$ Offer Auswertung < max_period $\cdots$ & -17 & \\
\hline 10 & *.* Offer Auswertung < max_period ... & -14 & \\
\hline 11 & ... Offer Auswertung < max_period ... & .20 & \\
\hline 12 & $\cdots$ Offer period < max period $\cdots$ & -26 & \\
\hline 13 & -.. Offer period < max_period $\cdots$ & .26 & \\
\hline 14 & $\cdots$ Offer period < max_period $\cdots$ & -26 & \\
\hline 15 & $\cdots$ Offer period < max_period $\cdots$ & -26 & \\
\hline 16 & ... Offer Auswertung < max_period ... & -4 & \\
\hline
\end{tabular}

Abb.5.13 Clients' Table

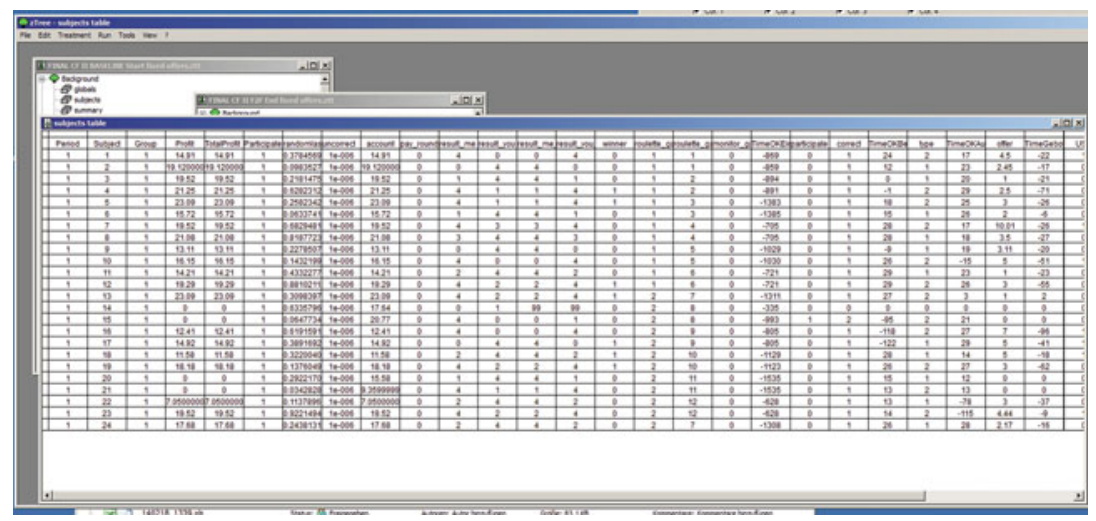

Abb. 5.14 Subjects Table 
Abb. 5.15 Wartebildschirm „Bitte warten Sie. Das Experiment geht weiter, sobald alle Teilnehmerinnen und Teilnehmer bereit sind“

Kommunikatives Handeln und die Akte seiner Erzeugung sind an den menschlichen Körper gebunden. Im Fall der verwendeten Software erfolgt die Objektivierung kommunikativen Handelns durch die Eingabegeräte, die die ProbandInnen nutzen müssen, um die geforderten Handlungen im Experiment auszuführen. Es zeigt sich, dass die Materialität, ihre Prägkraft und der Eigensinn (im Sinne einer Performativität) entscheidenden Einfluss auf das mit dem Handeln erwirkte ausüben. Basierend auf ihrer Programmierung und den Vorgaben der Forschenden, realisiert sich durch die technischen Medien eine Begrenzung der Eingabemöglichkeiten und des Handlungsspielraums der ProbandInnen. Die genutzten Medien tragen so zur Standardisierung der kommunikativen Inhalte bei. Im Fall der oben dargestellten Auswahl zwischen verschiedenen Lotterien (Abb. 5.18) müssen die ProbandInnen genau eine Lotterie durch Anklicken auswählen. Eine Mehrfachwahl wird von der Software nicht akzeptiert. Auch in Fällen in denen ein Gebot, 


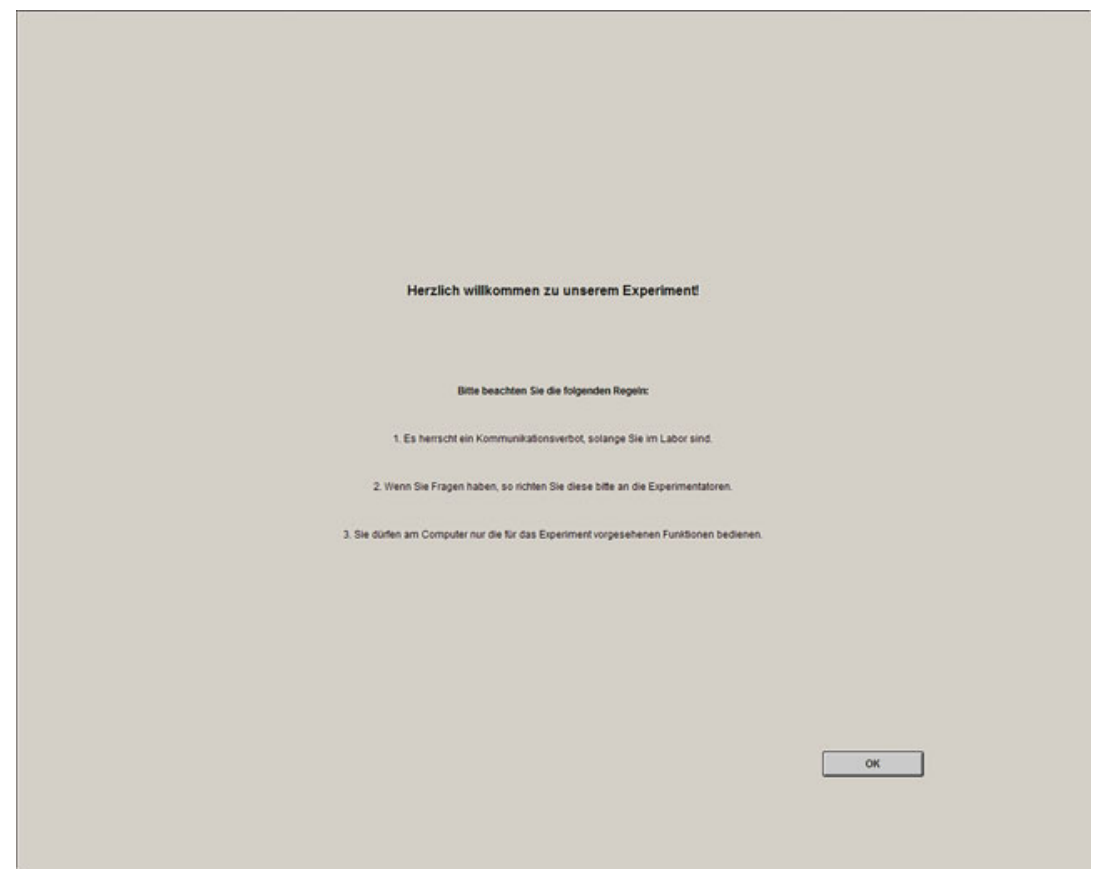

Abb. 5.16 Aktiver Bildschirm 1 - Information zur Kenntnis nehmen und „OK“-Button drücken

beispielsweise für ein Lotterielos, eingetragen werden muss (Abb. 5.17), sind nur bestimmte Eintragungen zulässig. Fehlermeldungen weisen darauf hin, wenn von den ProbandInnen Korrekturen vorgenommen werden müssen, um regelkonforme Eintragungen zu tätigen. So müssen Euro- und Cent-Beträge durch einen Punkt und nicht durch ein Komma voneinander getrennt werden, um als Eingabe von Programm angenommen zu werden.

Der Einsatz solcher Softwareprogramme zur standardisierten Datenerfassung in ökonomischen Experimenten ist selbst ein Standard in der Forschung und muss in den Publikationen von Forschungsergebnissen ausgewiesen werden. Sie sollen eine Standardisierung der Kommunikation mit den ProbandInnen erleichtern und helfen, die Abläufe im Labor zu überwachen (Fischbacher 2007). Durch diesen Einsatz von Medien im Labor wird eine Vermittlung kommunikativer Handlungen über den begrenzten Sozialraum der Arbeitskabinen hinaus realisiert. Die 


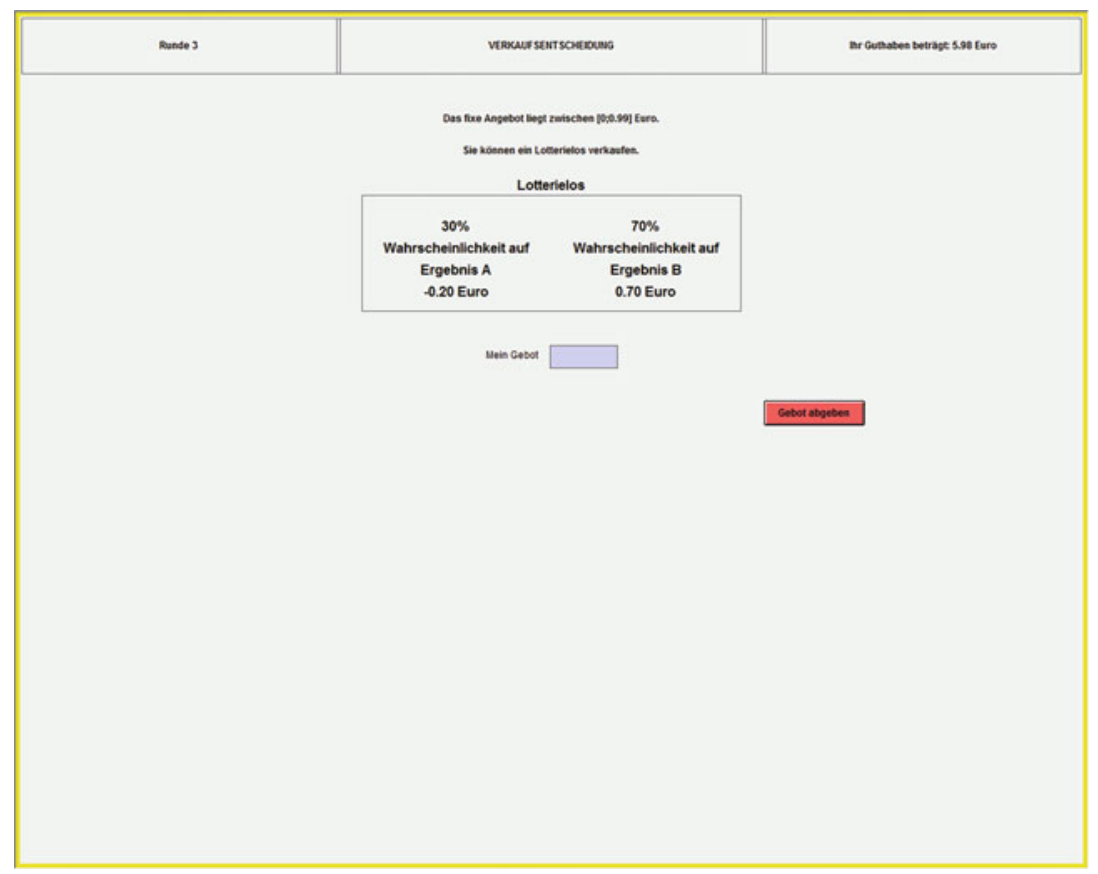

Abb.5.17 Aktiver Bildschirm 2 - Wert eintragen und „Gebot abgeben“-Button drücken

Übersichtsapplikationen (Abb. 5.13 und Abb. 5.14) ermöglichen es den Labormitgliedern trotz der visuellen Barrieren des Laborraums, die Handlungsfortschritte der ProbandInnen zu kontrollieren, ohne körperlich an die Arbeitskabinen der ProbandInnen herantreten $\mathrm{zu}$ müssen. Durch die Programmierung wird der sequenzielle Ablauf der einzelnen Handlungsschritte festgelegt, welchen die ProbandInnen bei der Datenerhebung durchlaufen. All diese Aspekte begünstigen die Standardisierung des Laborgeschehens und tragen so zum Doings einer spezifischen Laborsituation bei. Forschende berichteten zudem in den gemeinsamen Gesprächen, dass sie einen entscheidenden Vorteil im Einsatz der Software darin sehen, dass die Daten der Erhebungen nach Abschluss der Experimente direkt in gleichartiger und digitaler Form zur Verfügung stehen und für die Analyse genutzt werden können. Die Replikation einer Versuchsanordnung und die Zusammenfassung von unterschiedlichen Sessions einer Treatmentvariation kann durch die erneute Nutzung eines programmierten Treatments deutlich unterstützt 


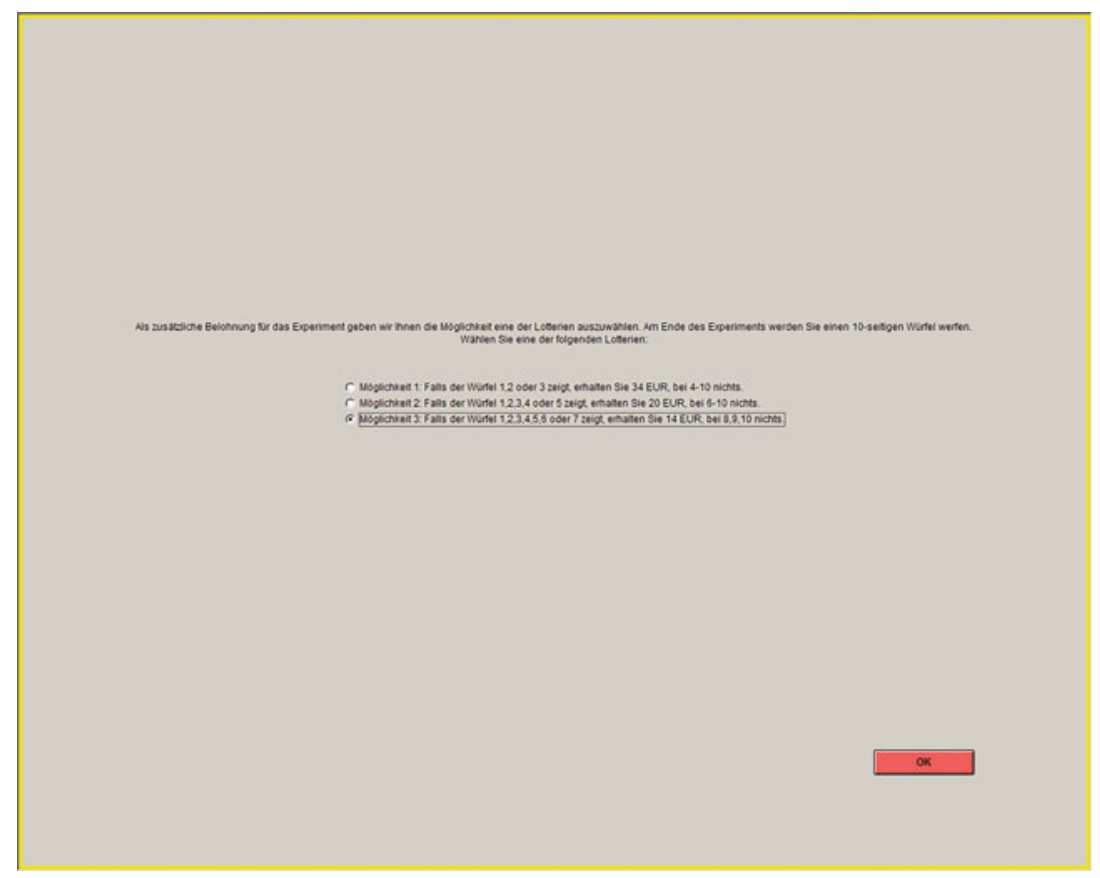

Abb.5.18 Aktiver Bildschirm 3 - Option auswählen und „OK““-Button drücken

werden. Auf technischer Ebene wird so das Konstanthalten der Randbedingungen in der Erhebungssituation befördert. Die Nutzung von spezifischen Softwareprogrammen ist damit nicht nur Ausdruck des Experimentmoduls innerhalb der Durchführung eines Experiments, sondern hat über diesen hinaus noch eine weitere Funktion.

Für die kommunikative Konstruktion des ökonomischen Laboratops ist die oben dargestellte Begrenzung des Handlungsspielraums durch die Eingabe- und Handlungsmöglichkeiten, die den ProbandInnen offengestellt werden, von zentraler Bedeutung. Sie befördert sowohl das Doing der rationalen und regelkonformen Verhaltenserwartungen als auch das Doing der spezifisch ökonomisch-rationalen Verhaltenserwartungen. Der Handlungsspielraum für kommunikatives Handeln ist durch die aktiven Bildschirme, je nach Rolle der ProbandInnen, unterschiedlich präfiguriert. Nur bestimmte Eingaben und Handlungen sind zulässig und 
werden vom Computerprogramm ohne Fehlermeldung akzeptiert. Auf rein technischer Ebene wird dadurch ein Zuwiderhandeln der ProbandInnen gegenüber den vorgegebenen Regeln der Forschenden dadurch erschwert. Dies führt zu einer technischen Verankerung des Spielmoduls: Die Regeln des Spiels werden nicht nur in sprachlicher oder schriftlicher Form, wie im Zuge der Begrüßung und in den Instruktionen, kommuniziert. Sie materialisieren sich hier vielmehr in aller Deutlichkeit durch die Darstellung der Spiel- und Entscheidungssituation und den Konsequenzen, welche sich durch diese ergeben. Die Probandinnen müssen agieren, zwischen Handlungsalternativen wählen oder Eingaben machen, die den vorgegebenen Parametern des Programms entsprechen. Es steht ihnen natürlich jederzeit frei, sich diesem Handlungszwang zu entziehen und das Labor zu verlassen. In diesem Fall laufen sie jedoch Gefahr, all ihren erspielten Gewinn zu verlieren. Die Art der Darstellung, der sich diese Software-Programme bedienen, ist zugleich Ausdruck des Ökonomiemoduls und verankert dieses. Die gängige Fokussierung auf Zahlen und monetäre Gewinne in den dargestellten Bildschirmoberflächen führt zu einer gezielten Betonung ökonomischer Aspekte der Handlungssituation. Das Spielmodul wird hier auf typische Weise als äußere Schale des Ökonomiemoduls verankert. Das Spiel, welches es zu spielen gilt, lässt sich auf die gegebenen Informationen reduzieren. Diese können so spartanisch sein, wie im Fall der obigen Abbildungen. Da ein regelkonformes Handeln von den Probandinnen eingefordert wird, wird ihnen zugleich angezeigt, dass diese Informationen für ihre Wahl oder Handlung eine ausreichende Grundlage bieten sollten. Es wird eine Rahmung der Situation induziert und nicht formuliert. Die Rahmung des Laboratops ist ein Weg der Fokussierung auf Aspekte des Entscheidungsverhaltens und seiner Beforschung, die aus Sicht der Forschenden für eben dieses relevant sind. Ohne dass den ProbandInnen offiziell mitgeteilt wird, dass es nun Zeit für quantifizierbares ökonomisches Entscheidungsverhalten ist, trägt die technische Vermittlung des kommunikativen Handelns einen entscheidenden Anteil an eben dieser Fokussierung.

Die kommunikativen Handlungen der ProbandInnen werden durch ihre Eingaben in Objektivationen überführt. Auch hier erfolgt eine gezielte Reduktion der vermittelten Inhalte. Durch die eingesetzte Mediation werden Objektivationen kommunikativer Handlungen produziert, die von den Forschenden anschließend als ökonomische Entscheidungen der ProbandInnen betrachtet werden können. Bilden mehrere ProbandInnen in einer Versuchsanordnung eine Spielergruppe, werden diese Objektivationen kommunikativer Handlungen einzelner ProbandInnen wiederum an andere SpielpartnerInnen vermittelt und wirken auf diese und ihr je eigenes kommunikatives Handeln und auf die Situationsrahmung (im Sinne 
des frame-in-use). Die technisch vermittelte Transformation der kommunikativen Handlungen in analysierbare Ergebnisse ist somit ein zentraler Baustein zur aktiven Produktion des Ökonomiemoduls. Die Prägkraft des eingesetzten technischen Mediums trägt hier entscheidend zur Verankerung und Verknüpfung der unterschiedlichen Moduln des ökonomischen Laboratops bei.

\subsubsection{Zusammenfassung der Ebene der Binnenstruktur}

Auf Ebene der Binnenstruktur wurden eine Reihe von unterschiedlichen Merkmalen thematisiert, die zur kommunikativen Konstruktion des ökonomischen Laboratops beitragen. Viele der dargestellten Aspekte zielen auf eine Standardisierung der Laborsituation und auf das Konstanthalten der Untersuchungsbedingungen für die ProbandInnen ab. Das Experimentmodul tritt hier als Rahmungselemente der Transformation deutlich hervor. Die materielle Struktur des Labors, die wiederholte Kommunikation der zentralen Verhaltensregeln, der Einsatz spezifischer Softwareprogramme und klare Gliederung des Handlungsprozesses, tragen maßgeblich zu einem Doing einer spezifischen Laborsituation bei und dienen der Aufrechterhaltung der Standards bei der Durchführung von Experimenten. Indem die Arbeitsplätze und die zur Verfügung gestellten Informationen in einem möglichst hohen Maße standardisiert werden, soll die Reproduzierbarkeit der Experimente unter gleichbleibenden Bedingungen für die ProbandInnen gesichert werden.

Die Verankerung des Spielmoduls erfolgt durch verschiedene Formen kommunikativen Handelns. In den Ausführungen zur standardisierten Begrüßung, wurde beispielsweise deutlich, dass die Art der Entäußerung der Regeln auch die Kommunikationsmacht ausdrückt, die den Labormitgliedern in dieser sozialen Situation zugesprochen wird. In Instruktionen und der einleitenden Begrüßung erfolgt die Explizierung der grundlegenden Verhaltensregeln auf schriftliche und mündliche Weise. Mittels weiterer typischer Formen kommunikativen Handelns wird ihre Durch- und Umsetzung auf performative Weise bestärkt. Die Instruktionen verwenden einfache Darstellungen der Spielsituation, die die aus Sicht der Forschenden relevanten Dimensionen und Aspekte erläutern. Die zentralen Verhaltensregeln, die unterschiedlichen Rollen und die Gewinnmodalitäten stehen dabei im Zentrum der Erklärung.

Die Instruktionen sind ein interessantes Beispiel für die unterschiedlichen Modalitäten. Im Labor zeigt sich ein Wechsel zwischen den unterschiedlichen Prägkräften, die ein Medium in der Vermittlung kommunikativer Inhalte 
ausübt. Hinsichtlich der Modalität der Kommunikation liegen sowohl am Bildschirm als auch in den Instruktionen die Inhalte primär in textlicher Form vor. Die Engführung auf das Sprachspiel der Forschenden ist im Fall der aktiven Bildschirmoberflächen jedoch deutlich gesteigert. Durch die Festlegung von Parametern für zulässige Eingaben wird der Handlungsspielraum der ProbandInnen deutlich reduziert.

Durch Beispiele oder die Durchführung von Quizzen wird in vielen Experimenten versucht, das angestrebte Text- und Regelverständnis der ProbandInnen zu überprüfen und zu verbessern. Wie oben dargestellt, erfüllt das materielle Setting eine grundlegende Funktion für die Schaffung einer Laborsituation und begünstigt das Doing regelkonformen Verhaltens der ProbandInnen. Die physische Vereinzelung der ProbandInnen durch die Kabinen unterstützt, dass die Möglichkeiten von Kommunikations- und Interaktionsbeziehungen begrenzt und reglementiert werden können.

Im Zuge der Darstellung binnenstruktureller Merkmale wurde darauf verwiesen, wie im Experimentallabor auf charakteristische Weise die unterschiedlichen Ebenen von Moduln verknüpft und ineinander verschachtelt werden. Die Engführung auf das Sprach- und Verhaltensspiel der Forschenden erfolgt dabei schrittweise. Die Prägkraft der kommunikativen Handlungen ist dabei unterschiedlich stark auf eine Übernahme der antizipierten Sprach- und Verhaltensspiele der Forschenden ausgerichtet. Am Beispiel der Lotterien wurde ausgeführt, dass, je nach untersuchter Fragestellung der Experimente, bestimmte Verfahren der Messbarmachung ökonomischen Entscheidens eingesetzt werden. Diese stellen selbst eine spezifische Verknüpfung von Experiment-, Spiel und Ökonomiemodul dar und verweisen auf die disziplinspezifischen paradigmatischen Annahmen der Wirtschaftsforschenden.

Die Betonung monetärer Gewinnmöglichkeiten und die Sanktionsandrohung des Geldverlustes, auf die beispielsweise in den Prä- und Postsequenzen oder in den Instruktionen des Experiments hingewiesen wird, tragen zum Doing ökonomisch-rationaler Verhaltenserwartungen bei und damit auch zur Rahmenwirksamkeit des Ökonomiemoduls. Die Fokussierung und Betonung dieser Aspekte wird auch durch die Bildschirmoberflächen deutlich, die den ProbandInnen präsentiert werden. An keiner Stelle wird den Teilnehmenden mittgeteilt, dass sie jetzt ökonomisch entscheiden müssten. Diese Art der Rahmung wird vielmehr induziert und Rahmungsalternativen durch Techniken der Reduktion der Sozialwelt geschwächt. Hier werden das Spiel- und Experimentmodul durch das Ökonomiemodul gleichsam bestärkt. Wie in den Ausführungen zur Außenstruktur dargestellt, ist entscheidungsabhängige Entlohnung ein grundlegendes Kriterium und ein Standard in wirtschaftswissenschaftlichen Laborexperimenten. 
Der in Aussicht gestellte Gewinn kommuniziert zugleich, was im Rahmen des Experiments als die allgemeine Zieldimension der Handlungen der ProbandInnen gilt. $^{58}$

Die Laborsituation ist eine multimodale Kommunikationssituation. Wie die Ausführungen zu den Gliederungsmerkmalen des Ablaufs im Labor darstellten, ist der Prozess des Laborexperiments durch eine Abfolge typischer Phasen charakterisiert, die kommunikative Handlungen auf verschiedene Weise vermitteln. Die zulässigen Arten der Interaktion sind in den unterschiedlichen Handlungsphasen dabei jeweils klar strukturiert und reglementiert. Den schriftlichen Instruktionen wird von den Forschenden hinsichtlich der Konstruktion der Entscheidungssituation ein zentraler Stellenwert beimessen. Wie die nachfolgenden Analysebeispiele auf der Ebene der situativen Realisierung aufzeigen, ist die Multimodalität der Kommunikationssituation im Labor für die Hervorbringung und Aufrechterhaltung seiner Rahmung elementar.

\subsection{Merkmale der situativen Realisierungsebene}

Im Analysekapitel der Binnenstruktur wurden anhand vielfältiger Beispiele Merkmale und Muster kommunikativer Handlungen im Labor betrachtet, die zur Standardisierung der Erhebungssituation und zum Konstanthalten der Randbedingungen beitragen. Die praktischen Methoden der Handelnden haben jedoch einen entscheidenden Anteil an der kommunikativen Konstruktion des ökonomischen Laboratops. Die situative Koordination des Handlungsablaufs sowie die Kontrolle und Sanktion von Regelüberschreitungen wird durch die ExperimentatorInnen realisiert. Allgemein lässt sich feststellen, dass für die ProbandInnen und Mitglieder des Laborteams im Ablauf des Laborexperiments verschiedene zulässige Möglichkeiten vorgesehen sind, kommunikative Handlungen im Labor zu vollziehen. Der Blick auf die Analyseebene der situativen Realisierung fokussiert solche typischen Formen kommunikativen Handelns, die die interaktive Produktion und Reproduktion der situativen Ordnung im Labor befördern und zur interaktiven Handlungskoordination der anwesenden Akteure beitragen.

\footnotetext{
${ }^{58}$ In Übereinstimmung mit den Erkenntnissen der verhaltensökonomischen Forschung wird anerkannt, dass ProbandInnen nicht ohne Weiteres als kühle Nutzenmaximierer im Sinne des Homo oeconomicus verstanden werden können. Es geht vielmehr darum, die Abweichungen und Anomalien rationalen Entscheidens, die sich in ihren Handlungen zeigen, durch systematische Beforschung zu erklären und so in das Modell ökonomischen Entscheidens zu integrieren.
} 


\subsubsection{Der Spielchip als Koordinationsobjekt der Platzzuweisung}

Betreten die ProbandInnen das Labor, besteht ihre erste Aufgabe darin, ihren Arbeitsplatz aufzusuchen. Bei der Betrachtung der Platzsuche der Teilnehmenden zeigen sich musterhafte Arten des Handlungsvollzugs. Die kommunikativen Handlungen, die die ProbandInnen während ihrer Platzsuche durch ihre Bewegungen vollziehen, sind nicht auf eine zentrierte Interaktion mit den anderen Anwesenden ausgerichtet. Auch wenn es sich bei der Platzsuche um eine Situation körperlicher Kopräsenz handelt, bewältigen die ProbandInnen ihre Platzsuche individuell. Die Situation der Platzsuche lässt sich im Sinne Goffmans als eine Situation „mit vielen Brennpunkten“ (Goffman 1973: 21) charakterisieren. Die Handelnden nehmen sich gegenseitig wahr. Sie nehmen auch aufeinander Rücksicht, sollten sie sich gegenseitig den Weg zu einem Arbeitsplatz versperren. Ihre Handlungen sind jedoch grundlegend auf die eigene Bewältigung der Aufgabe der Platzsuche ausgerichtet.

Wie im Analyseabschnitt der Binnenstruktur dargestellt, werden im beobachteten Labor für die Platzzuweisung der ProbandInnen nummerierte Spielchips verwendet. ${ }^{59}$ Für die Forschenden stellt die Verwendung der Spielchips ein standardisiertes Mittel dar, um den außenstrukturellen Anspruch der randomisierten Platzzuweisung im Labor umzusetzen. Für die ProbandInnen bildet der je spezifische Spielchip, den sie vor dem Betreten des Laborraum ziehen, ein entscheidendes Koordinationsobjekt für ihre Platzsuche im Labor. Um diesen Aspekt zu veranschaulichen wird im Folgenden noch einmal auf die Eingangssequenz zurückgegriffen, die zu Beginn des Analysekapitels dargestellt wurde.

In Reaktion auf eine Frage eines unidentifizierbaren Sprechers verweist der Experimentator Tim hier auf die koordinative Funktion des Spielchips für die Platzsuche der ProbandInnen.

\section{Z 6: Tim: GENAU, AUF DEN PLATZ SETZEN (.) MIT DER NUMMER AUF DEM CHIP}

Mit seiner Aussage verweist der Experimentator zudem auf den vermittelnden Zusammenhang zwischen dem gezogenen Spielchip und der Nummer der Arbeitskabine. Die Aufgabe, welche die ProbandInnen bewältigen müssen, ist es nun, die Arbeitskabine ausfindig zu machen, die die gleiche Nummer trägt wie ihr gezogener Spielchip. Ihr Spielchip und die auf diesem befindliche Nummer, ebenso wie

${ }^{59}$ Siehe hierzu Abschnitt 5.4.1. 
die Nummern an den Kabinen, besitzen somit eine kommunikative Funktion. Die Nummer auf dem Spielchip stellt in der Situation der Platzsuche eine Objektivation der Aufgabe der ProbandInnen dar und koordiniert situativ ihre Platzsuche im Labor. Für die kommunikative Konstruktion des ökonomischen Laboratops ist es zudem von entscheidender Bedeutung, dass die ProbandInnen den Spielchip selbstständig gezogen haben. Die Zufälligkeit ihrer Platzzuweisung wird so für sie selbst erfahrbar. Auch die ProbandInnen wissen um die Zufälligkeit ihrer Sitzplatzzuweisung und antizipieren diese als typischen Standard in der Durchführung der Laborexperimente. Anhand der Interaktion zwischen den Probandinnen Ina und Jana, die in der Eingangssequenz beschrieben wurde, soll dies illustriert werden.
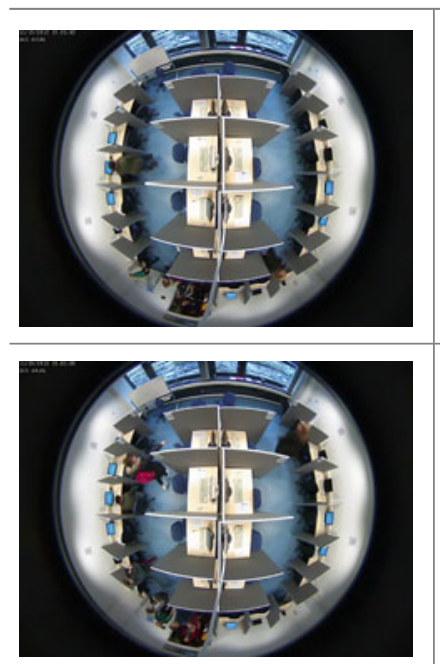

Die zwei Probandinnen Ina und Jana betreten das Labor. Jana richtet sich schon beim Einlaufen nach rechts aus. Sie läuft den ganzen rechten Gang entlang bis zur Fensterfront. Ina hingegen betritt den linken Gang. Sie wendet ihren Körper beim Laufen der mittleren Reihe der Arbeitsplätze zu.

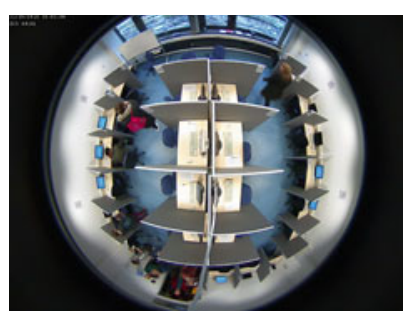

Im Anschluss daran knickt sie ihren rechten Arm ein, wendet ihren Kopf nach links und macht eine

Zeigegeste in die linke Richtung Arbeitsplatz Nummer 5. Sie kommentiert dies durch die Aussage: „Ach Gott, und ich bin hier."

Darauf blickt sie wieder zu Jana und sagt lachend:

„Das ist ja auch was.“ 


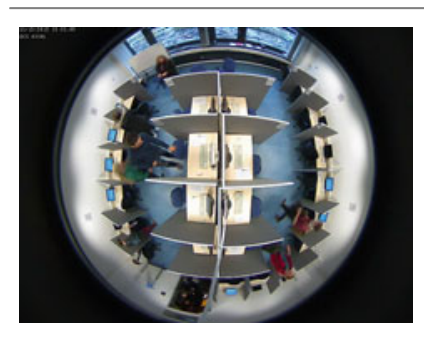

Ina wendet ihren Blick im Anschluss wieder von Jana ab und beginnt zugleich, ihren Rucksack abzusetzen. Sie stellt diesen an die Trennwand des Arbeitsplatzes. Jana blickt in Inas Richtung und läuft am Arbeitsplatz der ExperimentatorInnen vorbei und betritt von der Fensterseite aus den linken Gang.

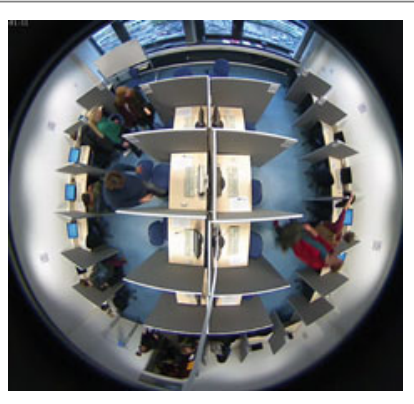

Jana wendet sich der oberen ersten Kabine mit dem Arbeitsplatz $20 \mathrm{zu}$, legt ihren Chip auf den Tisch und nimmt den dort befindlichen Stuhl ein. Sie wendet ihren Blick noch einmal kurz nach oben und scheint die Nummer dieses Arbeitsplatzes zu betrachten.

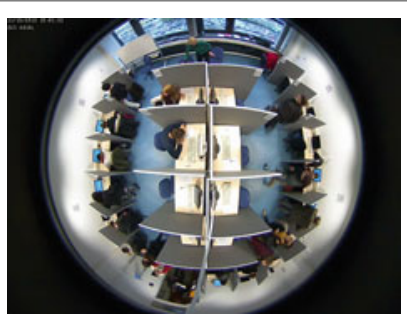

Jana zieht ihre Jacke aus und dreht sich zu Ina um. Diese hat ihre Blickrichtung jedoch auf das Innere der Kabine ausgerichtet. Jana hängt ihre Jacke über die Lehne ihres Stuhls und setzt sich auf diesen. Sie wendet ihren Körper zweimal leicht nach rechts und dreht sich dann noch einmal zu Ina um. Diese zeigt keine Reaktion.

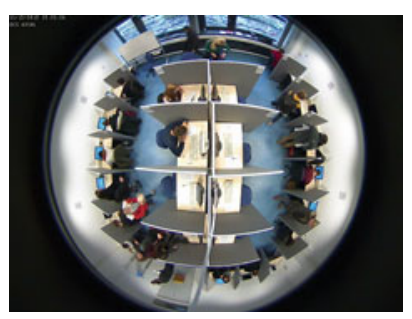

Jana nimmt darauf die auf ihrem Platz ausliegenden Instruktionen zur Hand und beginnt, darin zu lesen.

Diese kurze Interaktion zwischen den beiden Probandinnen legt die Interpretation nahe, dass die Teilnehmenden die Platzzuordnung durch den nummerierten Spielchip und die damit gegebene Handlungsanweisung verstehen, anerkennen 
und ihr durch ihre Handlungen Folge leisten. Auch wenn sich einige Teilnehmende kennen sollten, besitzen sie nicht die Möglichkeit, Arbeitsplätze auszuwählen, die direkt nebeneinanderliegen. Die Probandin Ina zeigt durch ihre verbale Äußerung, ,Ach Gott, und ich bin hier“ (Zeile 10), sowie daran anschlieBend mit, „das ist ja auch was“, und ihrer Intonation hier an, dass sie die räumlich nahe Positionierung der beiden Frauen als amüsant betrachtet. Es wird hier ersichtlich, dass auch die Probandin überrascht ist, dass sie so dicht beieinandersitzen. Die Probandin weiß somit darum, dass dies weder wahrscheinlich noch beeinflussbar ist. Die zufällige Zuweisung durch den Spielchip ist für sie ein erwartbares und nicht infrage zu stellendes Element der Experimentsituation. Es entäußert sich hier sowohl der im Zuge der Außenstruktur thematisierte Aspekt der Randomisierung und damit der zufälligen Verteilung der ProbandInnen im Labor sowie die Kenntnis, die auch die teilnehmenden ProbandInnen von diesem Merkmal der Laborsituation besitzen.

Das Ende der kurzen Handlungssequenz zwischen den Probandinnen Ina und Jana stellt exemplarisch dar, wie sich der Aufmerksamkeitsfokus der beiden Probandinnen verschiebt. Beide Probandinnen markieren hier deutlich das Ende der gemeinsamen Interaktionssequenz und den Übergang zu anderen Handlungen, wie dem Lesen der Instruktionen. Die soziale Situation des Miteinanders wandelt sich dabei zu einer Situation des Nebeneinanders. Im Fall der beiden Probandinnen ist der Wechsel von einer fokussierten Interaktion zu einer multizentrierten Interaktion im Vollzug der Platzsuche ersichtlich. Dass das Betreten des Labors und die Einnahme der Arbeitsplätze, mit der die Vereinzelung der ProbandInnen einhergeht, kann als ein typisches Merkmal im Vollzug der Experimente betrachtet werden. In enger Verknüpfung werden hier gleichsam Spiel- und Experimentalmodul verankert. Zur situativen Realisierung der Rahmenbildung im ökonomischen Laboratop trägt der nummerierte Spielchip maßgeblich bei, da er als Objektivation eine gezielte kommunikative Leistung erfüllt und den ProbandInnen anzeigt, welchen Arbeitsplatz im Labor sie individuell aufzusuchen haben.

Für die ProbandInnen ist das selbstständige Ziehen des Spielchips zugleich eine maßgebliche Voraussetzung für die Glaubwürdigkeit der Zufälligkeit der ihnen zugewiesenen Rollen und der zugesicherten Anonymität. ${ }^{60}$ Der Einsatz und die situative Nutzung der nummerierten Spielchips tragen in ihrer handlungskoordinativen Funktion zum Doing der spezifischen Laborsituation bei, da sie es den ProbandInnen auf standardisierte Weise ermöglichen, ihre zugewiesenen Arbeitsplätze aufzusuchen. Zugleich trägt der Vollzug der Platzsuche selbst

\footnotetext{
${ }^{60}$ Für weitere Ausführungen zum binnenstrukturellen Merkmal der standardisierten Platzsuche siehe Abschnitt 5.4.1.
} 
maßgeblich zur Vereinzelung der ProbandInnen und zum Herunterkühlen des sozialen Settings bei. Er befördert dabei das Doing von rationalen und regelkonformen Verhaltenserwartungen und kommuniziert den ProbandInnen gegenüber die Erwartungen, die an ihre Handlungen im Rahmen der Platzsuche gestellt werden.

Die Interaktion von Ina und Jana ist insofern ein Sonderfall, als dass die Probandinnen im Labor üblicherweise nicht mehr miteinander sprechen, sobald sie das Labor betreten. Dieser Ausschnitt wurde trotzdem für die Illustration herangezogen, da er verdeutlich, dass die Probandinnen die Zufälligkeit der Sitzplatzzuweisung als erwartbaren Aspekt des Laborexperiments antizipierten. Dem Kommunikationsverbot, das die ExperimentatorInnen im Zuge der einleitenden Belehrung als eine der zentralen Verhaltensregeln kommunizieren, entsprachen die ProbandInnen in den meisten beobachteten Experimenten bereits in vorauseilendem Gehorsam. Der nachfolgende Abschnitt wird die Differenz der Kommunikationsrechte zwischen ProbandInnen und Mitgliedern des Laborteams thematisieren.

\subsubsection{Kommunikationsrechte und Wege ihrer Erteilung}

Wie in den Ausführungen zu den Gliederungsmerkmalen des Laborexperiments angedeutet, unterscheiden sich die unterschiedlichen Phasen des Experiments hinsichtlich der vorgesehenen verbalsprachlichen Kommunikationsrechte der Beteiligten. Im Unterschied zu den anwesenden ProbandInnen ist es den Mitgliedern des Laborteams gestattet, im Labor verbal mit anderen anwesenden Personen zu kommunizieren. Doch auch in ihren kommunikativen Handlungen zeigen sich in den unterschiedlichen Handlungsphasen und -situationen verschiedene typische Kommunikationsmuster.

In der Durchführung der Experimente besteht eine Aufgabe der ExperimentatorInnen darin, den sequenziellen Ablauf der einzelnen Handlungsphasen im Experiment zu koordinieren. Unter Rückgriff auf ihre praktischen Sachkenntnisse und Handlungskompetenzen müssen sie bestimmen, wann der Abschluss einzelner Handlungskomplexe initiiert und zum nächsten Handlungskomplex übergegangen werden kann. Die unter Abschnitt 5.3.1 aufgeführten Gliederungsmerkmale des Laborgeschehens bilden jeweils eigenständige Handlungskomplexe.

Der Augenblick des Übergangs und der Initiierung einzelner Handlungsphasen oder -komplexe wird von einem Mitglied des Laborteams verbalsprachlich markiert. Ein Mitglied des Laborteams ergreift in diesen Situationen das Wort und 
richtet sich mit einem für alle Anwesenden hörbaren Redebeitrag an die ProbandInnen. Es wird den Teilnehmenden mitgeteilt, zu welchem Handlungskomplex im Folgenden übergegangen wird und auch ganz allgemein dargelegt, welche Aufgaben die ProbandInnen in diesem Handlungsschritt erwarten. Auf diese Weise wird zugleich der Abschluss der anderen Gliederungsschritte markiert. In vielen Fällen werden die ProbandInnen in diesen Übergangssituationen nicht nur darüber informiert, dass zum nächsten Handlungsschritt übergegangen werden soll, sondern auch, welche Arten kommunikativen Handelns in der folgenden Phase von ihnen erwartet werden bzw. zulässig sind. Die kommunikativen Handlungen der ExperimentatorInnen besitzen hier eine deutlich performative Wirkung. Sie objektivieren allen Anwesenden gegenüber, dass Handlungsphasen beendet sind bzw. beginnen. Es müssen hierbei die je spezifischen Rahmenbedingungen erfüllt sein, damit ein Übergang zur nächsten Phase des Handlungsprozesses erfolgreich durchgeführt werden kann. Die ExperimentatorInnen orientieren sich dabei an den für die spezifische Situation relevanten kommunikativen Handlungen und Objektivationen.

In den Phasen des Laborgeschehens, in denen die ProbandInnen mit den Benutzeroberflächen der Computer interagieren, ziehen die ExperimentatorInnen dazu vermehrt Objektivationen heran, welche durch den Übersichtsbildschirm verfügbar sind. Können die ExperimentatorInnen anhand des Übersichtsbildschirms nicht bestimmen, ob ein Übergang zwischen den Handlungsphasen möglich ist, werden die ProbandInnen gebeten, die Machbarkeit des Übergangs körperlich anzuzeigen. Dieser Aspekt wird im weiteren Verlauf der Darstellung durch den beschriebenen Übergang zwischen dem ,Lesen der Instruktionen" und dem ,Zeitfenster für Nachfragen' illustriert.

Im Vollzug des Experiments greifen die Mitglieder des Laborteams vermehrt auf typische sprachliche Phrasen oder Floskeln zurück, um das Ende von einzelnen Phasen des Handlungsablaufs einzuleiten und den Übergang zwischen ihnen zu markieren. Der Übergang zwischen dem Handlungskomplex des Eintreffens der ProbandInnen ${ }^{61}$ und ihrer Begrüßung wird beispielsweise durch eine Aussage wie die nachfolgende markiert: „So, herzlichen willkommen zum Experiment, wenn Sie nun alle Platz genommen haben, dann kann es losgehen“. Bei dem Übergang zwischen den Handlungsphasen zu Beginn der Bearbeitung der Aufgaben am Computer treten häufig Äußerungen auf wie: „Gut, wenn es jetzt keine Nachfragen mehr gibt, dann können wir mit dem Experiment beginnen“. Eine Entgegnung der ProbandIn ist in beiden Fällen nicht vorgesehen. Sie sind

\footnotetext{
${ }^{61}$ Dies entspricht dem im Abschnitt 5.3.1. ausgeführten Gliederungsmerkmal „1. Betreten des Labors durch die Teilnehmenden und Platzsuche“.
} 
hier nicht als SprecherInnen adressiert, sondern werden von dem Übergang der Handlungskomplexe in Kenntnis gesetzt.

In anderen Fällen richten die ExperimentatorInnen auch Redebeiträge an die ProbandInnen, die Möglichkeiten für eine regelkonforme Kommunikation mit ihnen bieten. Hier wird der Übergang zum nächsten Gliederungspunkt initiiert. Ob die Durchführung des Übergangs erfolgt, obliegt jedoch dem situativen Feedback der kommunikativen Handlungen der Anwesenden. Ein Beispiel dafür ist der Übergang vom ,Lesen der Instruktionen` zum ,Stellens von Nachfragen` zu den Instruktionen. In allen Experimenten erhalten die Teilnehmenden schriftliche Instruktionen und werden nach der Begrüßung gebeten, diese zu lesen. Wie oben dargestellt, besteht die Aufgabe der ExperimentatorInnen nun darin, situativ den angemessenen den Zeitpunkt für den Übergang zum nächsten Gliederungsaspekt zu bestimmen. Je nach Länge der Instruktionen warten die ExperimentatorInnen eine gewisse Zeit ab. Bezugnehmend auf ihre praktischen Erfahrungen wissen die Mitglieder des Laborteams, dass es typische körperliche Anzeichen dafür gibt, dass ProbandInnen das Lesen der Instruktionen abgeschlossen haben. Es zeigt sich hier häufig eine visuell erfassbare Veränderung der Körperposition der ProbandInnen. Während sie beim Lesen nach vorne über den Tisch gebeugt sind und ihren Kopf nach unten gewendet haben, nehmen viele ProbandInnen nach Abschluss der Aufgabe eine entspanntere Position ein. Sie richten sich etwas auf oder lehnen sich zurück und an die Stuhllehne an. Die ExperimentatorInnen können die veränderte Körperposition der ProbandInnen visuell erfassen. Entweder begnügen sie sich damit, diese aus der Position des Arbeitsplatzes der Labormitglieder zu beobachten oder sie schreiten die Gänge des Labors entlang. Die veränderte Körperpositionierung der ProbandInnen wird als Objektivierung für die Beendigung der Aufgabe des Lesens der Instruktionen betrachtet. Sie wirkt hier als kommunikative Handlung und wird zur Orientierung im Handlungsvollzug genutzt. Lässt sich nun bei einer Mehrzahl der sichtbaren ProbandInnen aufgrund ihre Köperhaltung vermuten, dass das Lesen der Instruktionen abgeschlossen ist, wird der Übergang zum nächsten Gliederungsaspekt initiiert. Da trotz der potentiellen Vagheit der Deutung körperlicher Zeichen gewährleistet werden muss, dass alle ProbandInnen das Lesen der Instruktionen abgeschlossen haben, schließt sich an die Beobachtung der veränderten Körperpositionierungen stets die Anwendung einer weiteren Ethnomethode an. Ein Mitglied des Laborteams fragt im Folgenden laut und für alle Anwesenden hörbar nach, ob es ProbandInnen gibt, die noch mehr Zeit zum Lesen der Instruktionen benötigen. Zudem wird den ProbandInnen mitgeteilt, dass sie dies bitte per Handzeichen anzeigen sollen. Auch hier werden die ProbandInnen somit deutlich darüber in Kenntnis gesetzt, welche Möglichkeiten für eine regelkonforme Interaktion in dieser Situation vorgesehen sind. Unter 
Berücksichtigung der visuellen Beschränkungen, die aufgrund der Raumstruktur im Labor vorliegen, kommunizieren die ExperimentatorInnen das Ergebnis ihrer Befragung stets deutlich hörbar für alle Anwesenden. Heben nach dem Stellen der Frage ProbandInnen ihre Hand, zeigen sie mit dieser kommunikativen Handlung an, dass die Handlungsphase des Lesens der Instruktionen noch nicht abgeschlossen werden kann. Mit einer entsprechenden Bemerkung wie „Gut, dann warten wir noch einen Augenblick“ wird vom Mitglied des Laborteams kommuniziert, dass es das kommunikative Handeln wahrgenommen hat. Die ExperimentatorIn macht das Ergebnis ihrer Wahrnehmung so zugleich auch für die anderen anwesenden ProbandInnen erfahrbar. Sollte keiner der anwesenden Teilnehmenden die Hand heben, so stellt dies ebenfalls einen Ausdruck kommunikativen Handelns dar. ${ }^{62}$ Die ProbandInnen signalisieren damit, dass sie alle das Lesen der Instruktionen abgeschlossen haben. In diesem Fall wird der Übergang zum nächsten Handlungsschritt ebenfalls sprachlich vollzogen, beispielsweise durch eine Aussage wie: „Gut, hat noch jemand Fragen zu den Instruktionen.“

Dass die Mitglieder des Laborteams den Übergang zwischen den einzelnen Handlungsphasen im Vollzug des Experiments koordinieren, trägt dabei zum Doing der spezifischen Laborsituation bei. Auf diese Weise wird der geplante sequenzielle Ablauf der einzelnen Phasen des Handlungsvollzugs koordiniert und umgesetzt. Zugleich gilt es immer, den geplanten sequenziellen Ablauf mit den situativen Gegebenheiten abzustimmen. Am Beispiel des Lesens der Instruktionen wurde deutlich, dass Handlungen der ProbandInnen für die Initiierung des Übergangs zwischen den Handlungskomplexen bedeutsam sind. Jedoch sind es die ExperimentatorInnen, die den spezifischen Zeitpunkt des Übergangs aktiv setzten und markieren. Im Prozess der Durchführung der Experimente ist hier eine deutliche Machtasymmetrie zwischen den beteiligten Akteuren angelegt. Die aktive Kontrolle und Koordination des sequentiellen Übergangs obliegt den Mitgliedern des Laborteams. Die koordinierenden Handlungen der ExperimentatorInnen und das aktive Initiieren und Anmoderieren der nachfolgenden Handlungsschritte tragen zudem deutlich zum Doing von regelkonformen Verhaltensweisen bei.

Dies liegt auch darin begründet, dass in ihrem Vollzug häufig auch die vorgesehenen und zulässigen Modi von Handlungen und Interaktionen kommuniziert werden, welche den ProbandInnen in der nächsten Handlungsphase zugestanden werden.

${ }^{62}$ Dies ist ein anschaulicher Beleg für Webers Definition des sozialen Handelns (1980), welche auch passive Formen des Handelns wie Dulden, Warten, Ausharren zu Formen sozialen Handelns erklärt. Das kommunikative Handeln, welches eine deutliche Wirkung auf die kommunikative Konstruktion der Handlungssituation besitzt, besteht ja in diesem Fall gerade darin, dass die ProbandInnen ,aktiv“ kein Handzeichen geben. 
Auch hier wird wieder die typische Verknüpfung von Experimental- und Spielmodul erzeugt. Die Standardisierung des Handlungsgeschehens ist Ausdruck des wissenschaftlichen Anspruchs des Konstanthaltens von Randbedingungen. Die geplante Umsetzung des standardisierten Ablaufs wird durch das performative Handeln der ExperimentatorInnen initiiert und unterstützt. Das Ungleichgewicht der Möglichkeiten der Einflussnahme auf eben dieses Handlungsgeschehen und die Akzeptanz von eben diesem ist eine Regel des Spiels im Experimentallabor. Die sozialen Rollen der Veranstaltung sehen hier vor, dass die einen die ,Durchführenden' sind und die anderen die ,Durchgeführten“.

\subsubsection{Fragen stellen und beantworten}

Das Kommunikationsverbot der ProbandInnen untereinander stellt eine zentrale Regel im Rahmen der Durchführung der Laborexperimente dar. Wie in den Ausführungen zur Binnenstruktur dargestellt, wird an verschiedenen Stellen des Ablaufs wiederholt auf diese Regel und die Relevanz ihrer Einhaltung verwiesen. ${ }^{63} \mathrm{Im}$ Zuge der Kontrolle über den Handlungsprozess im Labor besteht eine Aufgabe der Mitglieder des Laborteams darin, die verbale Kommunikation im Labor zu kontrollieren und zu koordinieren. Zur interaktiven Realisierung dieser Aufgabe greifen sie bei der Durchführung der Experimente auf typische Arten kommunikativer Handlungen zurück.

Die vorgesehene Art und Weise, in der die ProbandInnen in Interaktion mit den ExperimentatorInnen treten dürfen, ist auf eine spezifische Praxis des Fragestellens fokussiert. Das regelkonforme Initiieren einer Gesprächsinteraktion zwischen ProbandInnen und ExperimentatorInnen ist durch die Verhaltensregeln im Labor stark vorstrukturiert. Im Zuge der Begrüßung und in den schriftlichen Instruktionen werden die ProbandInnen darüber informiert, welche Handlungsschritte sie befolgen sollen, wenn sie eine Frage stellen möchten. Zugleich wird ihnen mitgeteilt, dass es untersagt ist, Fragen laut und für alle Anwesenden hörbar zu stellen.

Das nachfolgende Beispiel illustriert, welche Herausforderung die visuelle Begrenzung durch die Trennwände für die regelkonforme Initiierung des Fragestellens durch die ProbandInnen darstellen kann. Zudem wird ersichtlich, wie die Probandin die räumlich-materielle Begrenzung ihrer Arbeitskabine antizipiert und durch ihre kommunikativen Handlungen versucht, diese situativ und orientiert an den kommunizierten Handlungsbeschränkungen, zu überwinden.

\footnotetext{
${ }^{63}$ Siehe dazu insbesondere Abschnitt 5.3.3. und 5.3.4.
} 
Der dargestellte Ausschnitt zeigt die erste Runde des Entscheidungsspiels einer Session aus der Endowement-Effekt-Experimentserie. ${ }^{64}$ Die Begrüßung der ProbandInnen und das Lesen der Instruktionen sind bereits vollzogen und das Zeitfenster für Nachfragen zu den Instruktionen ist ebenfalls verstrichen. Die Probandin Katja sitzt in ihrer Kabine und schaut auf ihren Bildschirm.

Zwei Mitglieder des Laborteams tauchen hinter Katjas Kabine auf.
Leise flüsternd besprechen sie etwas. Kurz darauf verlässt der
Experimentator nach links den Bildausschnitt und die Experimentatorin
geht nach rechts.

\footnotetext{
${ }^{64}$ Die nachfolgenden Screenshots zeigen Aufnahmen aus der Perspektive einer Webcam, die am Bildschirm in der Arbeitskabine 18 angebracht wurde. Die Aufgabe der ProbandInnen in dieser Situation besteht darin, einen Preis festzulegen, zu dem sie bereit wären, ein in ihrem Besitz befindliches Lotterielos zu verkaufen.
} 

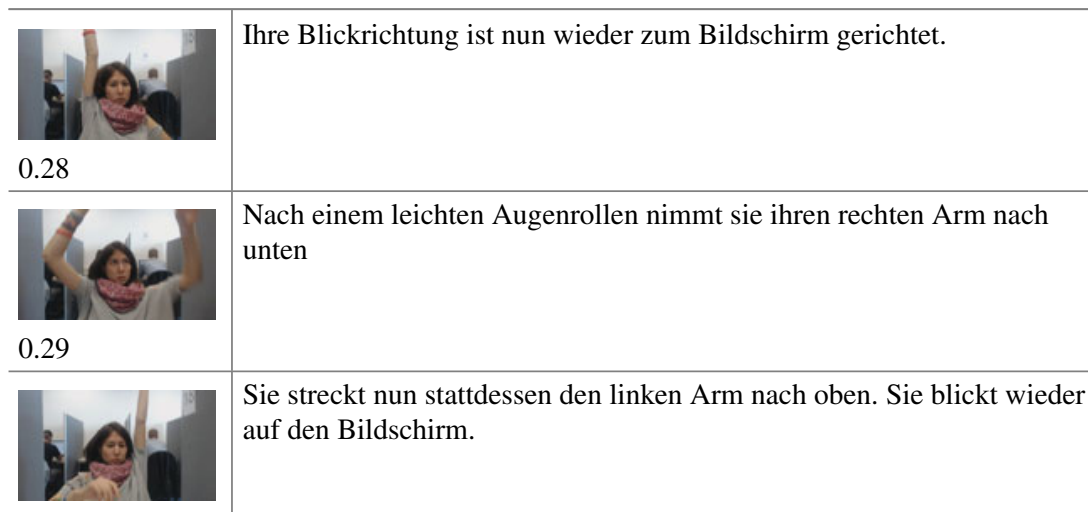

Sie streckt nun stattdessen den linken Arm nach oben. Sie blickt wieder auf den Bildschirm.

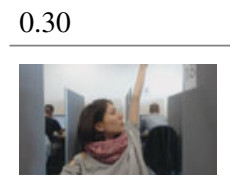

Wenige Augenblicke darauf wendet sie ihren Kopf wieder nach links.

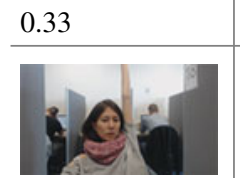

Sie wendet ihren Blick wieder in die Kabine hinein und sagt dabei leise: „Mann eh.“

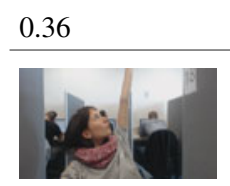

0.39

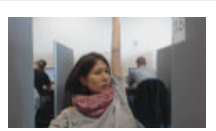

Sie wendet ihren Blick in die Kabine und hält ihren linken Arm weiterhin nach oben gestreckt.

0.43

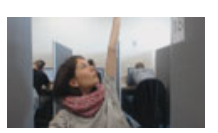

Noch einmal blickt sie an ihrem ausgestreckten Arm nach oben.

Sie schaut an ihrem linken Arm empor und strekt diesen durch.

Noch einmal blickt sie an ihrem ausgestreckten Arm 


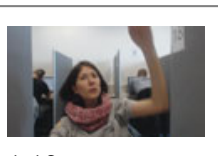

Wenig später lässt sie ihren linken Arm runtersinken

1.12

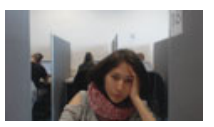

Sie stütz ihren Kopf auf ihre geöffnete Hand auf und schaut in Richtung des Bildschirms.

1.13

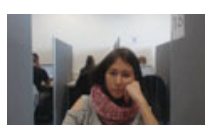

Sie richtet sich etwas auf, streicht sich mit der linken Hand durchs Haar und stützt ihren Kopf nun auf ihre linke Faust auf. Sie macht ein seufzendes Geräusch und schaut weiter auf den Bildschirm.

\subsection{6}

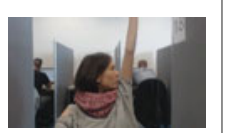

Sie hebt abermals den linken Arm und wendet ihren Kopf zugleich nach links.

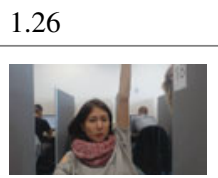

Sie wendet ihren Kopf in Richtung der Kabine. Kurz darauf ist die Experimentatorin im Bildausschnitt zu sehen.

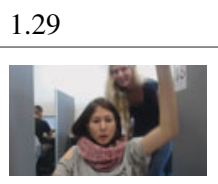

Mit dem Eintreten der Experimentatorin in ihre Kabine lässt Katja ihren Arm langsam runtersinken.

\subsection{0}

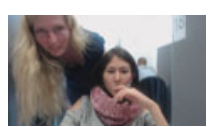

Nachdem die Experimentatorin Katjas Kabine betreten hat, blicken beide auf den Computerbildschirm. Mit leiser, flüsternder Stimme stellt Katja ihre Frage.

\subsection{2}

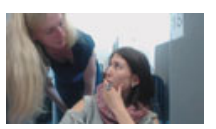

Die Experimentatorin benatwortet die Frage ebenfalls in leiser Sprechlautstärke und maßgeblich durch einen Verweis auf die am Bildschirm dargestellten Informationen.

Dieses Beispiel zeigt auf, wie die ProbandInnen die visuellen Barrieren der Arbeitskabinen überwinden müssen, um eine Interaktion mit den ExperimentatorInnen aufzunehmen. Wie aus der Darstellung ersichtlich wird, blickt die die 
Probandin Katja mehrfach an ihrem ausgestreckten Arm empor. In einigen Situationen zeigt sich, dass sie diesen im Anschluss noch ein Stück höher nach oben ausstreckt. Dies kann als deutliches Anzeichen dafür interpretiert werden, dass die Probandin hier die visuellen Barrieren durch die Trennwände in ihren Handlungen antizipiert. In der Art ihres kommunikativen Handelns zeigt sie an, dass sie bemüht ist, ihren Fragebedarf regelkonform anzuzeigen. Aufgrund der verbalen und nonverbalen Anzeichen, wie das Augenrollen oder die Aussage „Mann eh", liegt die Interpretation nahe, dass Katja die Wartezeit bis zum Erscheinen der Experimentatorin als störend lang empfindet. Sie weicht jedoch nicht von der regelkonformen Weise des Fragestellens ab. In der Frage-Antwort-Interaktion der beiden Frauen thematisiert Katja die lange Wartezeit nicht. Durch ihr Handeln zeigt sie hier ihre Kenntnis über die situativen Regeln des Fragestellens an. Sie beginnt erst zu sprechen, nachdem die Experimentatorin in ihre Kabine eingetreten ist und sich neben ihr positioniert hat. Ihre Sprechlautstärke ist sehr leise und verhalten. Auch die Experimentatorin spricht nur leise und flüsternd. Auch hinsichtlich der Beantwortung der Frage zeigt sich die Anwendung des typischen Musters kommunikativer Handlungen. Die Experimentatorin verweist zur Beantwortung der Frage auf die Informationen, die am Bildschirm zu sehen sind. Die auf der Benutzeroberfläche präsentierten Inhalte werden zur Beantwortung der Frage herangezogen. Als zusätzliche Information wird nur entäußert, dass nachfolgend Lotterien mit anderen Werten und Wahrscheinlichkeiten folgen werden und die erste Runde des Spiels der Illustration des Entscheidungsspiels dient.

Das Aufzeigen der ProbandInnen durch das Heben ihrer Hand stellt eine kommunikative Handlung dar und zugleich eine Objektivierung des regelkonformen Versuchs der Kontaktaufnahme des jeweiligen Versuchsteilnehmenden. Mittels dieser Objektivierung gelingt es, die Handlungen der Akteure entsprechend ihrer Rollen zu koordinieren. Sie bildet eine typische musterhafte Form kommunikativen Handelns der ProbandInnen. Die ExperimentatorInnen, die die Bedeutung dieser performativen Handlung kennen, werden so darüber in Kenntnis gesetzt, dass eine persönliche Interaktion und ein Zwiegespräch mit der jeweiligen ProbandIn initiiert werden soll. Die Aufgabe der anwesenden Labormitglieder besteht in der Antizipation dieser möglichen kommunikativen Handlungen. Sie halten während des Experiments und insbesondere während der Phase der Nachfragen zu den Instruktionen Ausschau nach ProbandInnen, die ihre Hand heben und so anzeigen, dass sie eine Frage stellen möchten. Sehen sie eine gehobene Hand, treten sie umgehend an den Platz des betreffenden Teilnehmenden heran, damit dieser seine Frage stellen oder sein Problem kommunizieren kann. Zeitliche Verzögerungen und damit Wartezeiten für einzelne ProbandInnen treten auf, wenn 
Labormitglieder gerade mit der Beantwortung der Fragen anderer Probandinnen befasst sind.

Im Zuge des Fragestellens einzelner ProbandInnen zeigt sich ein deutlicher Lautstärkewechsel in der sprachlichen Interaktion. Um dem paradigmatischen Anspruch der Kontrolle intendierter Störvariablen zu entsprechen, werden bei der Beantwortung von Nachfragen gezielt spezifische Ethnomethoden eingesetzt. Häufig wird im Zusammenhang mit der Beantwortung der Nachfragen auch das Attribut ,im Stillen“ verwendet. Zum einen treten die Mitglieder des Laborteams sehr dicht an die ProbandInnen heran und in die Arbeitskabine der jeweiligen Person ein. Zudem sind sie bemüht, die Lautstärke der Gesprächsinteraktion zu reduzieren. Dies erfolgt, indem das Mitglied des Laborteams selbst leise spricht. Sollte der fragende Teilnehmende seine eigene Gesprächslautstärke nicht ebenfalls deutlich reduzieren, so wird er darauf hingewiesen, dass er bitte gleichfalls leiser sprechen sollte, damit die anderen ProbandInnen nicht von seiner Nachfrage in Kenntnis gesetzt werden. Auch bei der Beantwortung der Frage zielen die ExperimentatorInnen darauf $a b$, die für das Treatment vorgesehenen Randbedingungen aufrechtzuerhalten. Sie nutzen aus diesem Grund die Informationen, die den ProbandInnen im Rahmen der Versuchsanordnung zur Verfügung gestellt werden. Wenn die Möglichkeit besteht, wird auf den Inhalt oder einzelne Abschnitte der schriftlichen Instruktionen verwiesen (siehe Abb. 5.19a und 5.19b) oder es werden die auf der Benutzeroberfläche präsentierten Informationen herangezogen. Nachfragen nach einer richtigen oder guten Strategie zur Bearbeitung der gestellten Aufgaben werden mit dem Verweis zurückgewiesen, dass es bei der Datenerhebung ja gerade um die Entscheidungen der einzelnen ProbandInnen gehen würde.

Abb. 5.19a Frage-Antwort Interaktion (Totale)

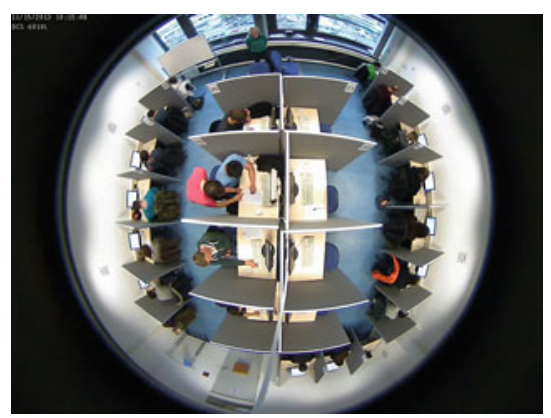




\section{Abb. 5.19b}

Frage-Antwort Interaktion (Zoom)

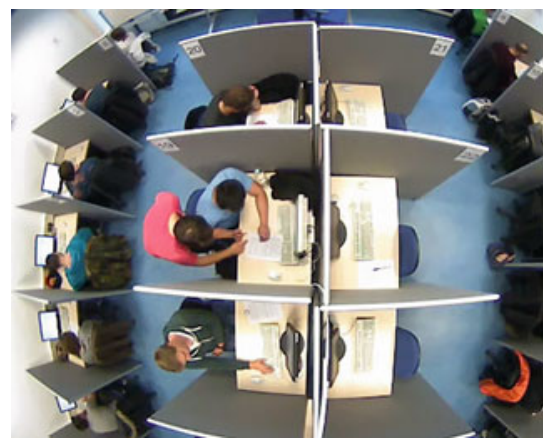

Die typischen kommunikativen Muster, die sich bei der Beantwortung von Nachfragen zeigen, sind somit stets darauf ausgerichtet, sowohl für die fragenden Versuchspersonen als auch für die anderen ProbandInnen die angestrebte und geplante Informationsgrundlage und Situationsdarstellung der Versuchsanordnung bestmöglich aufrechtzuerhalten. Es sollen durch die kommunikativen Handlungen der ExperimentatorInnen möglichst keine zusätzlichen Informationen oder Hinweise für mögliche Strategien gegeben werden, die die Handlungen der ProbandInnen ungewollt beeinflussen könnten.

Das obige Beispiel der Probandin Katja zeigt zugleich auf, dass die kommunikative Konstruktion des ökonomischen Laboratops nicht nur durch die kommunizierten Regeln und die Kontrolle der ExperimentatorInnen entsteht. Auch die ProbandInnen tragen durch ihre kommunikativen Handlungen maßgeblich zur aktiven Produktion und Reproduktion des ökonomischen Laboratops bei. Sie orientieren sich an den vorgegebenen begrenzten Handlungsspielräumen und -möglichkeiten und richten ihr kommunikatives Handeln an diesen aus. Das Accounting des Spielmoduls besteht genau darin, dass auch die ProbandInnen nach den vorgegebenen Regeln agieren. Die musterhaften kommunikativen Handlungen der ProbandInnen müssen aus diesem Grund ebenfalls als Ethnomethoden betrachtet werden, die das Doing von rationalen und regelkonformen Verhaltenserwartungen im ökonomischen Laboratop bestärken.

Auch wenn die sprachliche Interaktion zwischen den ProbandInnen untersagt ist, so zeigen sich in der Durchführung der Experimente durchaus zulässige Formen kommunikativer Handlungen und Interaktionen zwischen den ProbandInnen. Diese sind jedoch in den meisten Fällen ${ }^{65}$ technisch vermittelt. Durch den

\footnotetext{
${ }^{65}$ Eine direkte face-to-face Interaktion zwischen den ProbandInnen ist in den beobachteten Experimenten nur dann zulässig gewesen, wenn sie selbst Teil der Treatmentvariation
} 
Einsatz der genutzten Medien und durch die spezifische Programmierung von Benutzeroberflächen, mit denen die ProbandInnen interagieren, sind ihre Handlungsspielräume stark begrenzt. Eine ausführlichere Darstellung dieses Aspekts erfolgt weiter unter im Abschnitt zur technisch vermittelten Interaktion.

\subsubsection{Der Übersichtsbildschirm der Experimentatoren}

Wie in den Ausführungen über den Laborraum ausgeführt, verfügen die ExperimentatorInnen über einen separaten Arbeitsplatz an der Fensterseite des Labors, an dem sich zwei Computerbildschirme befinden. Die für die Durchführung des Experiments benötigten Programme werden von dort aus gestartet. Von den ExperimentatorInnen wurde in den beobachteten Experimenten stets das Programm z-Tree verwendet. Dieses unterstützte sie nicht nur bei der sequenziellen Organisation der Handlungen der Experimentteilnehmenden (siehe unten), sondern bot ihnen auch einen Überblick über die jeweiligen Handlungenschritte der einzelnen ProbandInnen. Die folgenden Ausführungen und die nachfolgenden Beispiele werden die zentrale Bedeutung aufzeigen, die das interaktive Zusammenspiel der unterschiedlichen Ethnomethoden der Labormitglieder im Labor besitzt.

Das zentrale Tool zur Koordinierung der unterschiedlichen Ethnomethoden der Labormitglieder ist der Übersichtsbildschirm „Clients' Table“ des Softwareprogramms. Dieser bieten den Experimentatoren einen Überblick darüber, welchen Handlungsschritt die ProbandInnen im Labor gerade vollziehen.

In der linken Spalte (Markierung 1) werden dabei die Anzahl der beteiligten ProbandInnen im durchgeführten Experiment angegeben. Als „Clients“ werden die Computer bezeichnet, die im Labor durch die ProbandInnen im Rahmen der Session genutzt werden. Auf diesen wird die Nutzerapplikation ,z-leaf“" ausgeführt und über das Netzwerk mit dem Computer der ExperimentatorInnen verbunden. Im Beispielfall (Abb. 5.20.) sind somit 16 ProbandInnen-Computer, über das Netzwerk vermittelt, in das Programm eingebunden. Die Übereinstimmung der Clients mit den nummerierten Arbeitsplätzen im Labor liegt jedoch nur vor, wenn alle Arbeitsplätze im Labor besetzt sind. Wenn dies nicht der Fall ist und die ExperimentatorInnen in der Vorbereitung des Experiments einzelne Arbeitsplätze auswählen, erfolgt eine andere Zuordnung der Clients zu den

des durchgeführten Experiments war. So wurde beispielsweise in der Experimentserie Endowment-Effekt-USB-Stick in einer Phase der Sessions stets das Spiel Vier-Gewinnt von zwei anwesenden ProbandInnen gespielt. In einer Treatmentvariation wurde anonym und vermittelt über das Netzwerk gespielt. In einer zweiten Treatmentvariation hingegen spielten zwei nebeneinandersitzende ProbandInnen in einer face-to-face Situation. 


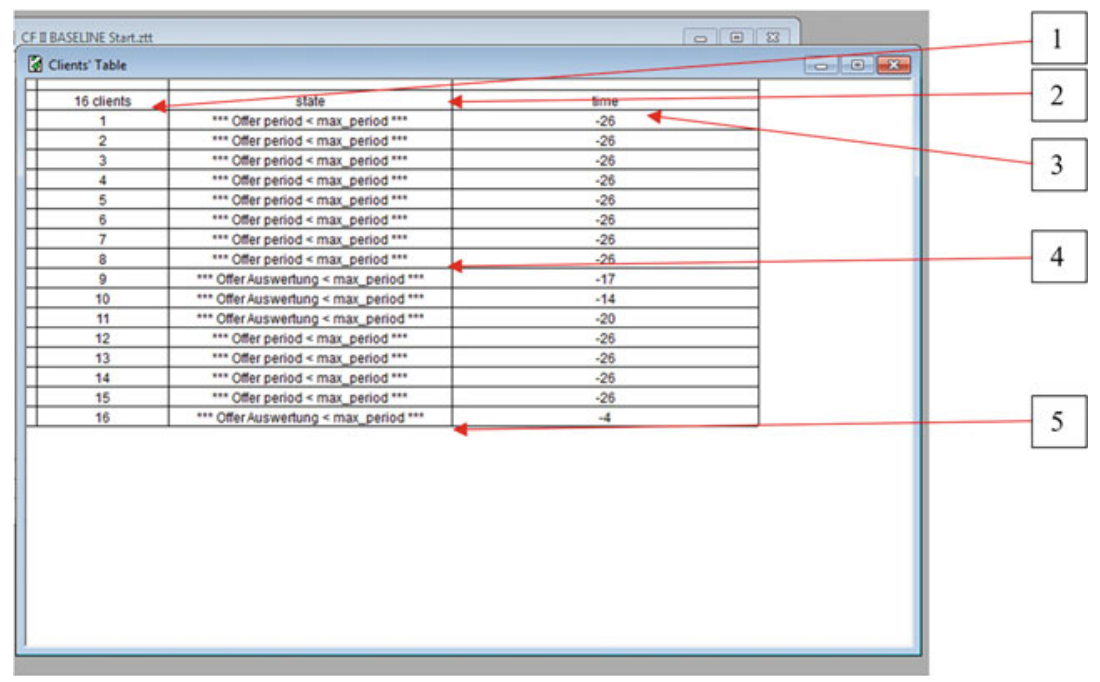

Abb. 5.20 Clients' Table mit Markierung

Arbeitsplätzen. Die aktiven Arbeitsplätze im Labor werden mittels eines anderen Programms bestimmt. Den Zusammenhang zwischen der Subjektnummer bei zTree und den Arbeitsplätzen im Labor können die Forschenden bei Bedarf mittels eines Vergleichs zweier Tabellen einsehen.

Die mittlere Spalte der Clients” Table trägt den Namen ,state“ (Markierung 2). Diese gibt Auskunft über die Benutzeroberflächen, die die ProbandInnen gerade an ihren Computern sehen und über ihren Bearbeitungsstatus. Die Benennung der einzelnen Benutzeroberflächen wird bereits im Zuge der Programmierung festgelegt. Im abgebildeten Fall handelt es sich um eine Runde des bereits erwähnten Lotteriespiels, in welchem die ProbandInnen den Kaufpreis für ein Lotterielos festlegen müssen. Wie die Abbildung 5.20 zeigt, können die ProbandInnen zwei unterschiedliche Benutzeroberflächen angezeigt bekommen „*** Offer period<max_period ***“ (z. B. Markierung 4) und ,„*** Offer Auswertung<max_period **** (z. B. Markierung 5). Die ExperimentatorInnen können sich durch einen Blick auf die Clients' Table darüber informieren, dass einige ProbandInnen gerade ihr Gebot setzen „*** Offer period<max_period ***“ und anderen TeilnehmerInnen bereits durch die Benutzeroberfläche ,*** Offer Auswertung < max_period $* * *$ " angezeigt wird, welches Ergebnis sie durch ihre Handlung erzielt haben. Die „***“ geben wiederum an, ob die Teilnehmenden mit 
der Bearbeitung dieser Benutzeroberfläche befasst sind oder bereits einen Wartebildschirm sehen. Im Fall einer passiven Benutzeroberfläche würde beispielsweise die Anzeige $\gg>>$ Offer Auswertung $<$ max_period $<<<$ erfolgen.

Die rechte Spalte der Clients' Table „time“ (Markierung 3) gibt Auskunft darüber, wie lange die ProbandInnen die gerade angezeigte Benutzeroberfläche bereits sehen. Durch die Programmierung kann hier eine maximale Bearbeitungsbzw. Anzeigezeit von Bildschirmoberflächen festgelegt werden. In den beobachteten Experimenten wurde diese Funktion des Programms jedoch nicht genutzt.

Damit die Mitglieder des Laborteams überblicken können, in welcher Periode sie sich bei einem Spiel über mehrere Runden befinden, können sie zudem Einblick in die „Subjects' Table“ (Abb. 5.21) nehmen. Hier werden alle Daten festgehalten, die mit den einzelnen Clients bzw. Forschungssubjekten verbunden sind. Alle Eingaben, rollenspezifischen Parameter und die aktuell erspielten Gewinne der einzelnen Forschungssubjekte werden hier simultan mit dem Ablauf im Labor dokumentiert. Für die Handlungskoordination im Labor sind die vielfältigen Informationen, die aus dieser Tabelle abgelesen werden können, jedoch meist nicht von Belang. ${ }^{66}$ In rundenbasierten Spielen ist es jedoch nötig, die Fortschritte in der ersten Spalte der Tabelle zu verfolgen. Hier wird unter der Variable „Period“ (Markierung 6) ausgewiesen, in welcher Runde eines Spiels sich die ProbandInnen gerade befinden. Auf diese Weise können die ExperimentatorInnen den zeitlichen Ablauf im Verlauf der einzelnen Sessions verfolgen und sich bemühen, zeitliche Engpässe mit nachfolgenden Sessions vorausschauend zu vermeiden, indem sie bei Bedarf ergänzende Ethnomethoden zur Beschleunigung der ProbandInnen einsetzen. Wie dies konkret erfolgt, wird weiter unten aufgezeigt.

Für die situative Realisierung bei der Durchführung der Experimente sind diese Übersichtsbildschirme von entscheidender Bedeutung: Sie ermöglichen es den ExperimentatorInnen, trotz der visuellen Beschränkungen eine Übersicht und Kontrolle über den Bearbeitungsstand der Aufgaben im Labor zu erhalten, ohne den ProbandInnen das Gefühl einer direkteren Überwachung ihrer Handlungen zu geben. Kenntnisse über den aktuellen Arbeitsstand aus diesen Tabellen zu gewinnen, ist für die Mitglieder des Laborteams jedoch nur möglich, da sie das entsprechende Wissen über den geplanten Ablauf des Experiments besitzen und die vermittelten kommunikativen Inhalte in ihrer Zeichenhaftigkeit verstehen können. Ohne Kenntnis der Symbolbedeutung von ,***“ und „>>>“ ist es beispielsweise nicht ersichtlich, welcher Bearbeitungsstand gerade vorliegt. Die Nutzung dieser Übersichtsbildschirme stellt somit eine Ethnomethode der

\footnotetext{
${ }^{66}$ Rein aus Interesse verfolgen die ExperimentatorInnen jedoch häufig das Geschehen im Labor über die Subjects Table.
} 


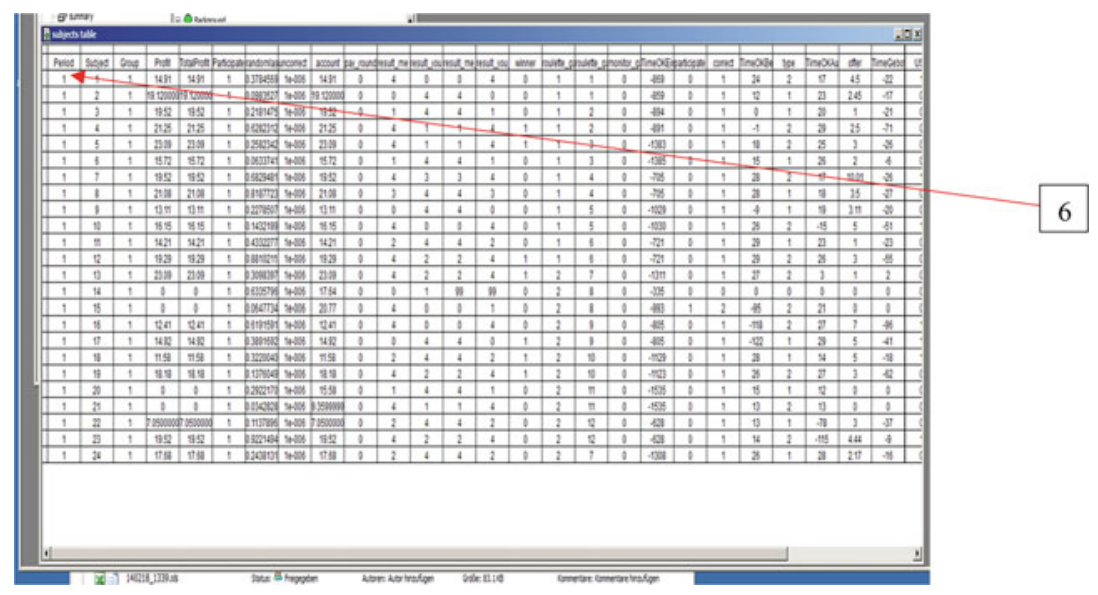

Abb.5.21 Subjects' Table mit Markierung

Labormitglieder dar und basiert auf ihren spezifischen Wissensbeständen über das durchgeführte Experiment und die Bedeutungen der vermittelten kommunikativen Inhalte der Software. Diese Ethnomethode befördert das Doing der rationalen und regelkonformen Verhaltenserwartungen, da es den Labormitgliedern ermöglicht, die Durchführung der Experimente in ihrem Ablauf detailliert zu kontrollieren und festzustellen, ob ihrerseits eingegriffen werden muss. Die handlungskoordinative Wirkung, die die Betrachtung und das Auslesen der Übersichtsbildschirme besitzt, besteht jedoch in der gezielten Verbindung mit anderen Ethnomethoden der Labormitglieder. Die in den nachfolgenden Kapiteln aufgeführten Beispiele greifen vielfach auf einen Abgleich mit einem der Übersichtsbildschirme zurück oder werden durch diesen initialisiert.

\subsubsection{Technische Realisierung des sequentiellen Ablaufs}

Wie in der Binnenstruktur im Abschnitt zur Mediation der kommunikativen Handlungen (Abschnitt 5.3.8.) dargestellt, werden zur Datenerhebung in wirtschaftswissenschaftlichen Experimenten Computer eingesetzt, an denen die ProbandInnen Eingaben tätigen müssen. Das Ziel des Einsatzes der Softwareprogramme besteht darin, die Kommunikation mit den ProbandInnen so weit wie möglich zu standardisieren und die ExperimentatorInnen bei der Kontrolle und 
geplanten Durchführung der Sessions der Experimente zu unterstützen. Zugleich liegen die erhobenen Daten bereits in digitaler Form vor, was die weitere Analyse erleichtert.

In diesem Zusammenhang wird nun dargestellt, wie der Einsatz der Programme zur sequentiellen Organisation der Abläufe während der Phase der Datenerhebung beiträgt. In einem ersten Schritt wird ein Beispiel herangezogen, welches die sequenzielle Organisation der Handlungen der ProbandInnen am Computer auf gleichartige Weise koordiniert. Das zweite Beispiel zeigt auf, wie durch den Einsatz der Software die Handlungen von ProbandInnen koordiniert werden, die unterschiedliche Rollen in der Durchführung eines Experiments einnehmen.

Durch die genutzten Medien des Computers und der Eingabegeräte ergeben sich gewisse Beschränkungen des Handlungsspielraums der ProbandInnen. Die Auswahl und Präsentation der Inhalte, die zulässigen Eingaben und die Möglichkeiten von Kommunikation und Interaktion werden jedoch in der Konzeption und Vorbereitung der Experimente von den Forschenden ${ }^{67}$ definiert. Diese Vorgaben und Handlungsbeschränkungen üben eine Prägkraft auf die kommunikativen Handlungen und die aus ihnen hervorgehenden Objektivationen aus. Die situative Handlungskoordination der ProbandInnen erfolgt dabei durch die präsentierten aktiven und inaktiven Benutzeroberflächen, mit denen sie interagieren sollen.

$\mathrm{Ob}$ alle ProbandInnen im Labor die gleichen aktiven Benutzeroberflächen präsentiert bekommen, hängt vom spezifischen Experiment ab. Nur wenn den TeilnehmerInnen im Rahmen des Experiments keine unterschiedlichen Rollen zugewiesen werden, erhalten sie alle die gleiche Eingabemaske. Da wirtschaftswissenschaftliche Experimente entscheidungsabhängig entlohnt werden, sind die Konsequenzen, die sich aus ihren Handlungen ergeben, jedoch nicht bei allen ProbandInnen gleich. Im nachfolgenden Beispiel wird diese Differenz illustriert.

Die unten dargestellten Benutzeroberflächen entstammen einem Treatment der Experimentserie zum Thema des Endowment-Effekts (,Endowment-Effekt-USBStick“). Nach der Begrüßung der ProbandInnen, dem Lesen der Instruktionen, der Beantwortung von Nachfragen und einer anschließenden Erklärung des Kaufsbzw. Verkaufsmechanismus durch ein Mitglied des Laborteams wurde allen ProbandInnen ein Entscheidungsspiel präsentiert. In diesem waren sie in Besitz eines Lotterieloses und sollten angeben, zu welchem minimalen Preis sie bereit wären, dieses zu verkaufen. Dieses Spiel wurde 8 Runden lang wiederholt und als

\footnotetext{
${ }^{67}$ Technische Mitarbeiter unterstützen diesen Prozess häufig und erstellen die Programmierung und die Benutzeroberflächen nach den Vorgaben und in Rücksprache mit den Forschenden.
} 
Verkaufsentscheidung bezeichnet. Im Anschluss spielten sie weitere 8 Runden ein Entscheidungsspiel, welches als Kaufentscheidungen deklariert wurden. Hier sollten die Teilnehmenden einen Preis festlegen, zu dem sie ein angebotenes Lotterielos kaufen würden. ${ }^{68}$ Die Werte der Lotterielose änderten sich von Runde zu Runde. Ziel dieser Spielrunden war es, mit den ProbandInnen das ,rationale Bewerten ' von Kauf- und Verkaufsgütern zu ,trainieren'. In den Instruktionen und den nachfolgenden öffentlichen Erklärungen einer ExperimentatorIn wurde den ProbandInnen mitgeteilt, dass ihr eingetragener Kaufs- bzw. Verkaufspreis mit einem zufällig generierten fixen Angebotspreis verglichen werden würde. Der hier eingesetzte Vergleich der eigenen Gebote mit einem zufällig generierten fixen Angebot entspricht dem Becker-DeGroot-Marschak-Mechanismus (BDM-Mechanismus). Dieses eingesetzte Verfahren sollte den ProbandInnen verdeutlichen, dass es strategisch für sie nicht vorteilhaft ist, einen Preis für den Kauf- bzw. Verkauf anzugeben, der von ihrer eigenen Bewertung abweicht. Durch die 16 Spielrunden sollten die ProbandInnen ,lernen', ihren eigenen Schwellenwert zu bestimmen, bei dem das Lotterielos ihnen genauso viel wert ist wie der Kauf- oder Verkaufspreis. den sie dafür angeben. ${ }^{69}$

Die Abbildung 5.22 zeigt die dritte Runde dieses Spiels, in welchem die Lotterielose von den ProbandInnen verkauft werden konnten. Allen im Labor befindlichen TeilnehmerInnen wurde zeitgleich diese Benutzeroberfläche angezeigt. Die einzige Differenz bestand im angezeigten erspielten Guthaben der ProbandInnen (Markierung 1).

\footnotetext{
${ }^{68}$ Hier musste in das Kästchen „Mein Gebot“ der maximale Preis eingetragen werden, zu dem die ProbandInnen bereit waren, das Lotterielos zu kaufen. Den Teilnehmenden wurden hier im Zuge des Spiels unterschiedliche Rollen (Käufer und Verkäufer) zugewiesen, doch diese Rollen waren in den 16 Runden bei allen Teilnehmenden gleich. Die jeweilige Spielrunde begann deshalb für alle ProbandInnen gleichartig.

${ }^{69}$ Der „BDM-Mechanismus“ gilt als ein anreizkompatibler Mechanismus der Präferenzmessung (Becker et al. 1964). Das Angebot, das die ProbandInnen abgeben, ist dabei stets verbindlich. Wenn der von ihnen angebotene minimale Verkaufspreis unterhalb oder gleich dem fixen Angebotspreis ist, wird ihr Lotterielos verkauft und sie erhalten den Betrag des fixen Angebots. Bei Kaufentscheidungen wird immer dann ein Los von ihnen gekauft, wenn der von ihnen gebotene maximale Kaufpreis genauso hoch oder höher als das fixe Angebot liegt. In diesen Fällen kaufen sie das Los zum fixen Angebotspreis. Da der Vergleichspreis (das fixe Angebot) zufällig bestimmt wird und nicht vom Wert des Gutes abhängt, welches gekauft bzw. verkauft wird, ist es aus der Perspektive eines nutzenmaximierenden, rational und strategisch handelnden Akteurs nicht vorteilhaft, einen anderen Wert zu wählen als den, der der eigenen Bewertung des Gutes entspricht. Anreizkompatibel bedeutet hier, dass es sich für die ProbandInnen hinsichtlich der Gewinne, die sie erspielen können, nicht auszahlt, einen anderen Wert anzugeben als ihren subjektiven Schwellenwert des Gutes.
} 


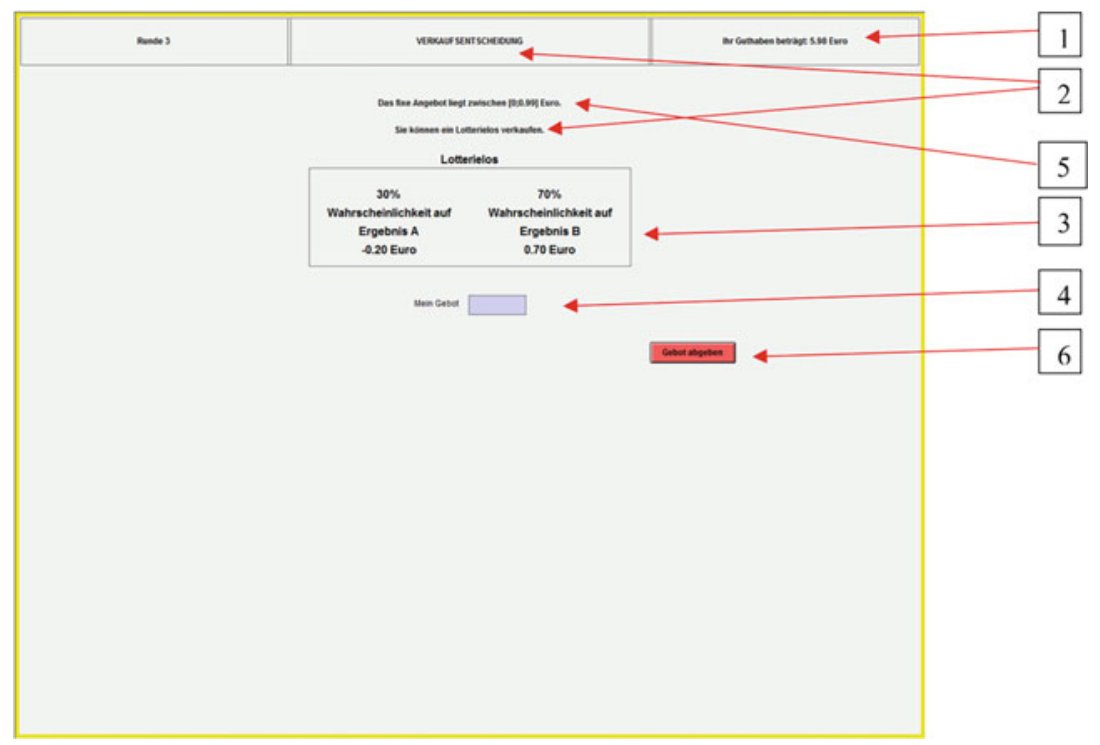

Abb. 5.22 Spielsituation Veraufsentscheidung

Den ProbandInnen wird hier eine Spielsituation präsentiert, in der sie ein Lotterielos besitzen und dieses verkaufen können (Markierung 2). Wenn sie das Lotterielos nicht verkaufen, wird es am Ende der Runde ausgespielt und führt zu Ergebnis A oder B (Markierung 3). Ganz wie oben in den Ausführungen zur Lotterie (Abschnitt 5.3.6) beschrieben, sind die jeweiligen Eintrittswahrscheinlichkeiten für Ergebnis A und B angegeben. Die ProbandInnen sollen nun den minimalen Wert bestimmen, ab dem sie bereit wären, das Lotterielos zu verkaufen. Diesen sollen sie in das Kästchen „Mein Gebot“ (Markierung 4) eintragen. Ihr abgegebenes Gebot wird mit einem zufällig bestimmten Wert (dem fixen Angebot) verglichen. Auf der Benutzeroberfläche wird angegeben, dass das fixe Angebot zwischen 0 Euro und 0.99 Euro liegen wird (Markierung 5). Zum Abschluss müssen sie auf den roten Button „Gebot abgeben“ (Markierung 6) klicken, um ihre Eingabe zu bestätigen. 
Wie oben dargestellt, werden aufgrund der vorher festgelegten Parameter die Spielergebnisse bestimmt. Im Fall dieses Spiels werden die eingetragenen Verkaufspreise mit dem fixen Angebot verglichen. ${ }^{70}$ Je nach Eintragung in das Kästchen „Mein Gebot“ erscheinen auf der folgenden Benutzeroberfläche unterschiedliche Ergebnisse. Im abgebildeten Beispielfall beträgt das fixe Angebot 0.13 Euro. Im abgebildeten Fall (Abb. 5.23), lag der minimale Verkaufspreis bei 0.10 Euro. Der eingetragene minimale Verkaufspreis ist in diesem Fall niedriger als der gezogene fixe Angebotspreis, deshalb wird das Lotterielos verkauft. Der fixe Angebotspreis wird in diesem Fall dem Guthaben der Versuchsperson gutgeschrieben.

Ist der eingetragene minimale Verkaufspreis jedoch höher als gezogene fixe Angebotspreis, so erfolgt kein Verkauf des Lotterieloses. Diesen Fall zeigt Abbildung 5.24. Hier wurde als minimaler Verkaufspreis 0.50 Euro festgelegt. Die Lotterie wird am Ende der Runde ausgespielt und Ergebnis A oder B treten ein und werden mit dem Guthaben der ProbandIn verrechnet.

Wie oben ausgeführt stellt die Anwendung der Spieltheorie und ihrer Modelle einen zentralen Forschungsgegenstand für viele wirtschaftswissenschaftliche Experimente dar. Als interaktive Entscheidungstheorie werden in experimentellen Untersuchungen häufig Situationen simuliert, in denen mehrere Personen im Labor als Spielpartner interagieren. In Fällen, in denen den ProbandInnen im Labor unterschiedliche Rollen zugeordnet werden, sind mit den Rollen auch unterschiedliche geforderte Aktivitäten verbunden. Diese werden in einer festgelegten sequenziellen Ordnung nacheinander durchlaufen und durch die unterschiedlichen aktiven Benutzeroberflächen den ProbandInnen entsprechend ihrer Rollen präsentiert. Die Handlungskoordination der Teilnehmenden ist dabei technisch durch die Programmierung vorstrukturiert. Das nachfolgende Beispiel illustriert die sequentielle Organisation der Interaktion einer Spielergruppe.

Im Fall des Mindestlohn-Experiments bestand eine Spielergruppe beispielsweise jeweils aus fünf ProbandInnen. In jeder Gruppe gab es drei Rollen (Konsument, Firma und Arbeitnehmer), von denen die ProbandInnen je eine zufällig zugewiesen bekamen. Jede Spielergruppe bestand aus einem Konsumenten, zwei Firmen und jeweils einem zugeordneten Arbeitnehmer pro Firma. Die Rollen

\footnotetext{
${ }^{70}$ Dieser Wert wurde vor Beginn der Experimente durch die ExperimentatorInnen bestimmt. Die ProbandInnen hatten jedoch keine Kenntnis über den Wert. Ihnen gegenüber wurde nachdrücklich in den Instruktionen und in der öffentlichen Erklärung im Labor darauf verwiesen, dass es sich um einen zufälligen Wert handelt und jeder Cent-Betrag zwischen 0 Euro und 0,99 Euro gleich wahrscheinlich ist. Die Spanne, aus der die fixen Angebotspreise ausgewählt wurden, war dabei in den einzelnen Spielrunden unterschiedlich hoch.
} 


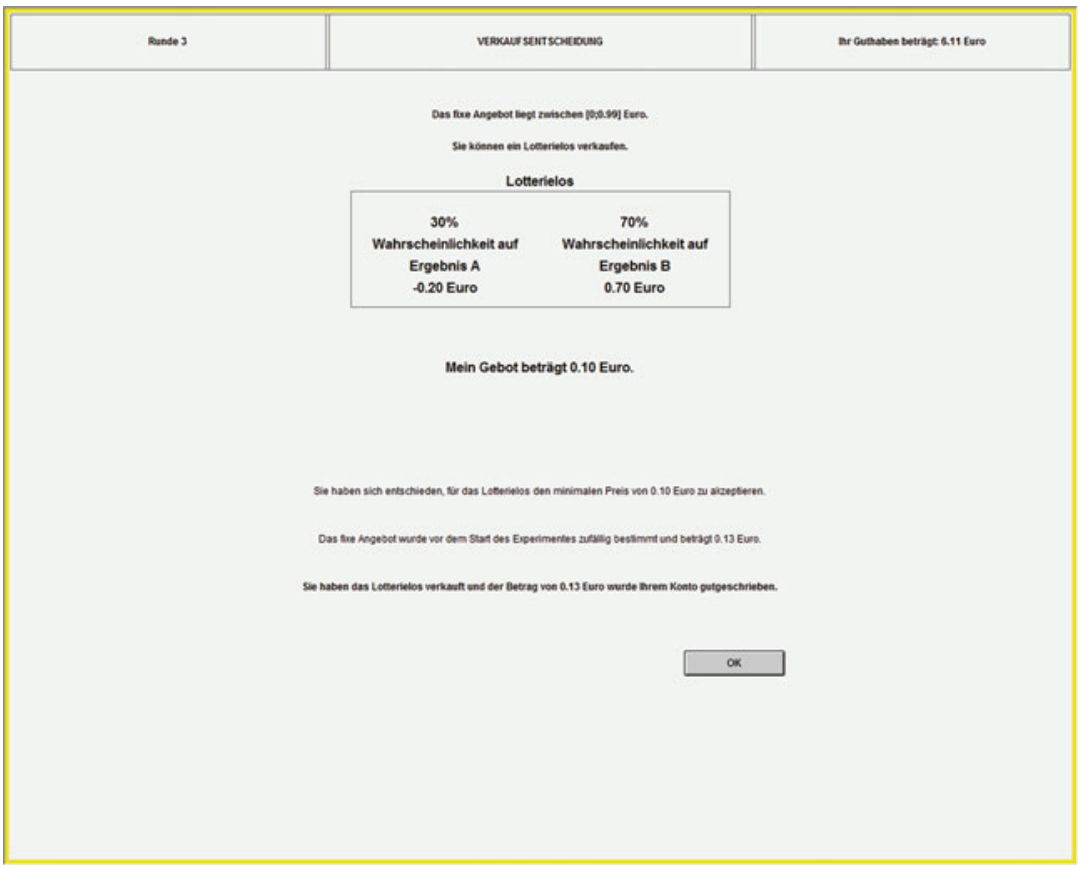

Abb.5.23 Ergebnis Verkaufsentscheidung

der Teilnehmenden blieben über das ganze Experiment gleich. Die beteiligten ProbandInnen interagierten dabei, vermittelt über das Computernetzwerk, und blieben füreinander anonym. Die Gruppe spielte insgesamt über 40 aufeinanderfolgende Runden miteinander, wobei ab der Runde 20 eine Änderung der Regeln, nicht jedoch der Rollenzuteilung eingeführt wurde.

Der Konsument verfügte in jeder Runde über einen fixen Geldbetrag von 250 Token, den er für den Kauf von 10 Produkten einsetzen konnte. Alles, was er nicht ausgab, durfte er als Gewinn selbst behalten. Jede Spielrunde begann damit, dass diejenigen ProbandInnen, die die Rolle der Firmen hatten, die Preise festlegen mussten, zu dem sie ihre Produkte anbieten wollten. Zudem mussten sie bestimmen, zu welchem Preis sie ihre Produkte anbieten würden, ohne vorher zu wissen, welchen Produktpreis ihr Konkurrent wählen würde. Darüber hinaus legten die Firmen fest, wie viel Lohn sie ihren Arbeitnehmern pro verkauftem 


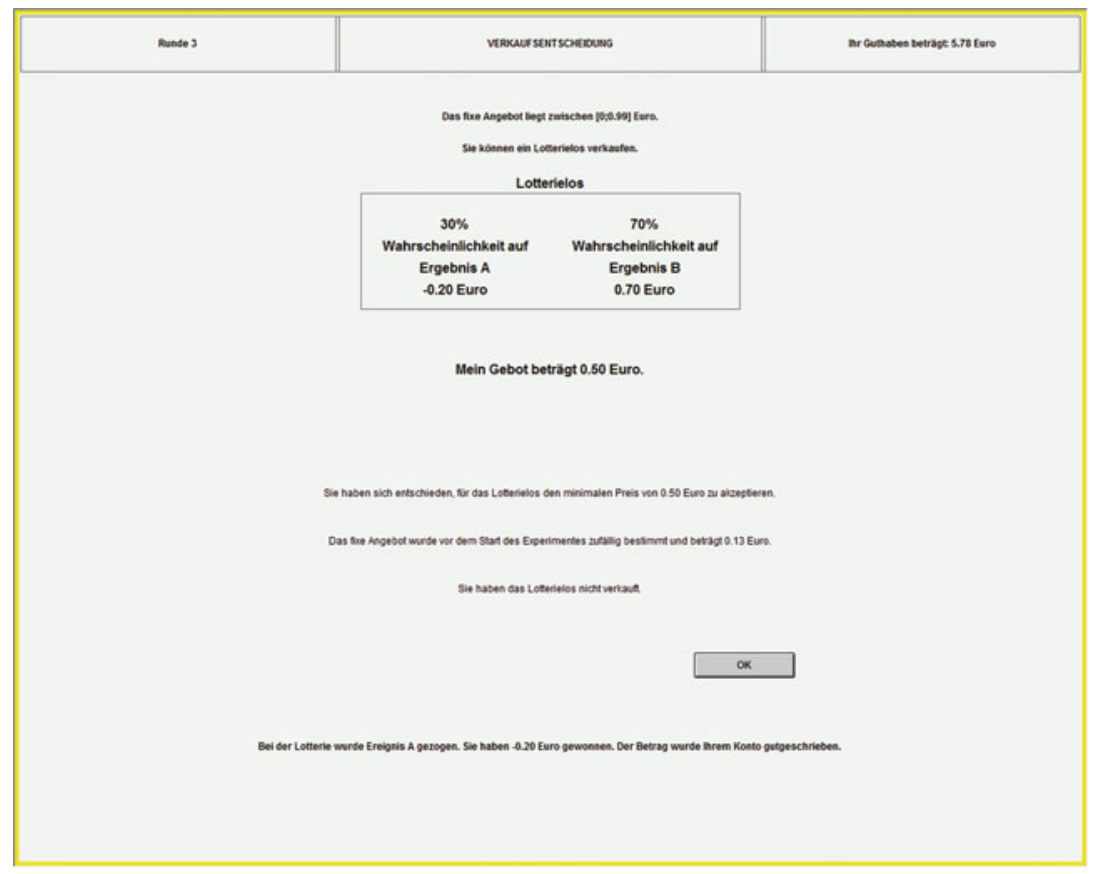

Abb.5.24 Ergebnis Verkaufsentscheidung 2

Produkt zahlen würden. Der Konsument konnte vor seiner Kaufentscheidung einsehen, welchen Preis die einzelnen Firmen festgelegt hatten und wie viel Lohn sie ihren Mitarbeiter zahlen würden. Darauf aufbauend entschied er, wie viele Produkte er von welcher Firma kaufen wollte. Am Ende jeder Wertungsrunde wurden die Entscheidungen und die Auszahlungen allen GruppenteilnehmerInnen angezeigt und mussten durch das Klicken eines OK-Buttons bestätigt werden.

In jeder Spielrunde zeigt sich somit eine klare sequenzielle Struktur, die durch die aktiven Benutzeroberflächen der jeweiligen ProbandInnengruppe koordiniert wurde. Die ersten aktiven Eingaben wurden von den ProbandInnen getätigt, denen die Rolle der Firmen zugewiesen wurde (Abb. 5.25). Die anderen ProbandInnen der Spielgruppe (Arbeitsnehmer und Konsumenten) mussten solange warten.

Die Ergebnisse der Eintragungen der Firmen A und B wurden im Folgenden dem Versuchsteilnehmenden präsentiert, der die Rolle des Konsumenten zugewiesen bekam. Auf einer aktiven Benutzeroberfläche sollte dieser eintragen, wie viele 


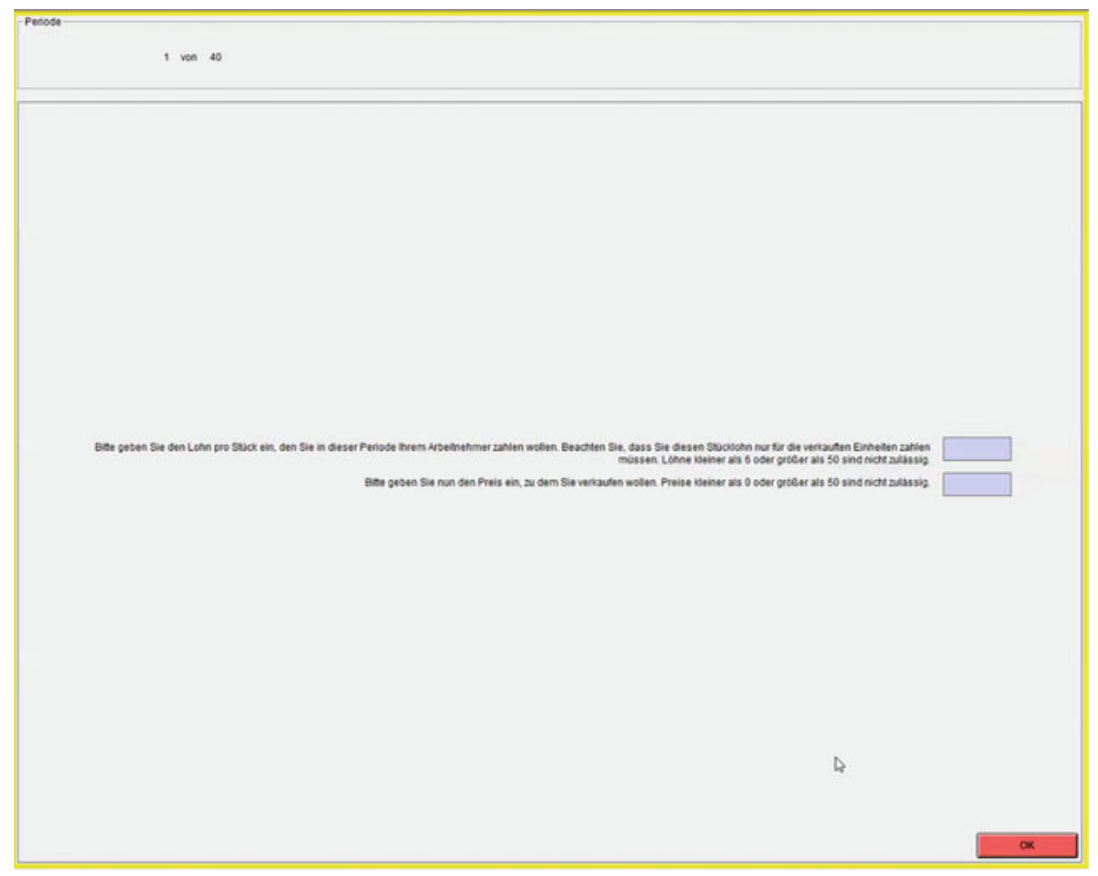

Abb. 5.25 Benutzeroberfläche für ProbandInnen in der Rolle der Firma

Produkte er von welcher Firma erwerben wollte (Abb. 5.26). Während er dies tat, wurde den anderen Mitgliedern seiner Spielgruppe (Arbeitsnehmern und Firmen) eine passive Benutzeroberfläche angezeigt, die sie zum Warten aufforderte.

Zum Abschluss wurden allen Beteiligten der Spielergruppe auf einer aktiven Benutzeroberfläche die Ergebnisse der Runde angezeigt (Abb. 5.27). Diese mussten mit einem Drücken des OK-Buttons bestätigt werden. Hatten alle im Labor befindlichen Spielergruppen diesen Ablauf vollzogen, so begann für alle ProbandInnen im Labor gleichzeitig die nächste Spielrunde.

Die zeitliche Abfolge der einzelnen Handlungsschritte innerhalb einer Spielgruppe folgt dabei einer klaren Ablaufstruktur. Wie in den obigen Abbildungen dargestellt, wird die Sequenzialität der Handlungsschritte der ProbandInnen somit durch die Bearbeitung unterschiedlicher Benutzeroberflächen realisiert. Begrenzt durch die Vorgaben des Programms, haben die ProbandInnen, entsprechend ihrer 


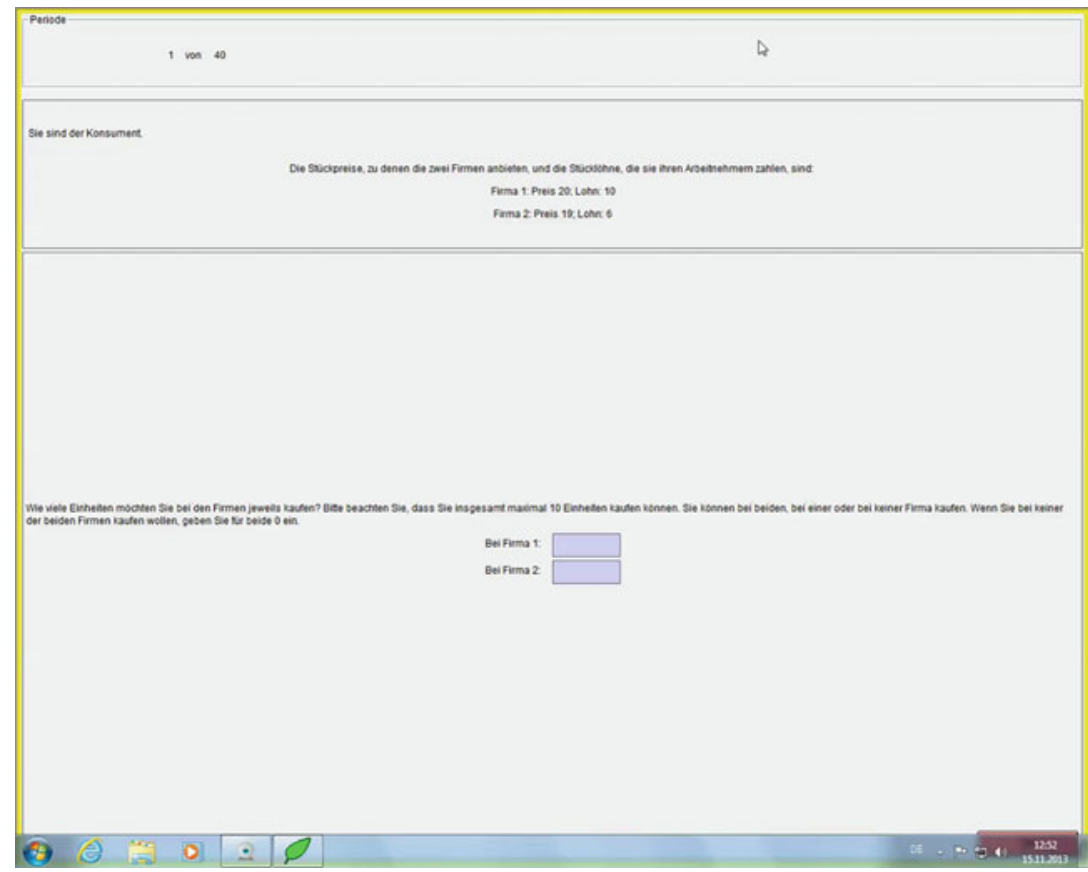

Abb.5.26 Benutzeroberfläche für ProbandInnen in der Rolle des Konsumenten

zugewiesenen Rollen bestimmte Aktivitäts- und Eingabemöglichkeiten. Die Wartezeit der einzelnen ProbandInnen variiert und ist auch davon abhängig, wie lange sie selbst für die Bearbeitung einzelner Handlungsschritte benötigten. Der Wechsel von einer Spielrunde zur nächsten oder zu einem nachfolgenden Experimentabschnitt erfolgt jedoch stets für alle ProbandInnen im Labor gleichzeitig.

Wie die Beispiele aufzeigen, hat der Einsatz dieser speziellen Softwareprogramme einen entscheidenden Einfluss auf die Handlungskoordination des Laborgeschehens. Die sequenzielle Abfolge einzelner Handlungsphasen wird durch die Programmierung festgelegt und realisiert sich in der Durchführung der Sessions im Labor. Die ProbandInnen erleben diese als eine Veraktung von einzelnen Handlungsphasen und als Sequenzketten einzelner Spielrunden. Diese zeichnen sich durch den charakteristischen Wechsel von Bildschirmoberflächen aus, welche sie zu verschiedenen Aktivitäten auffordern. Die Verankerung 


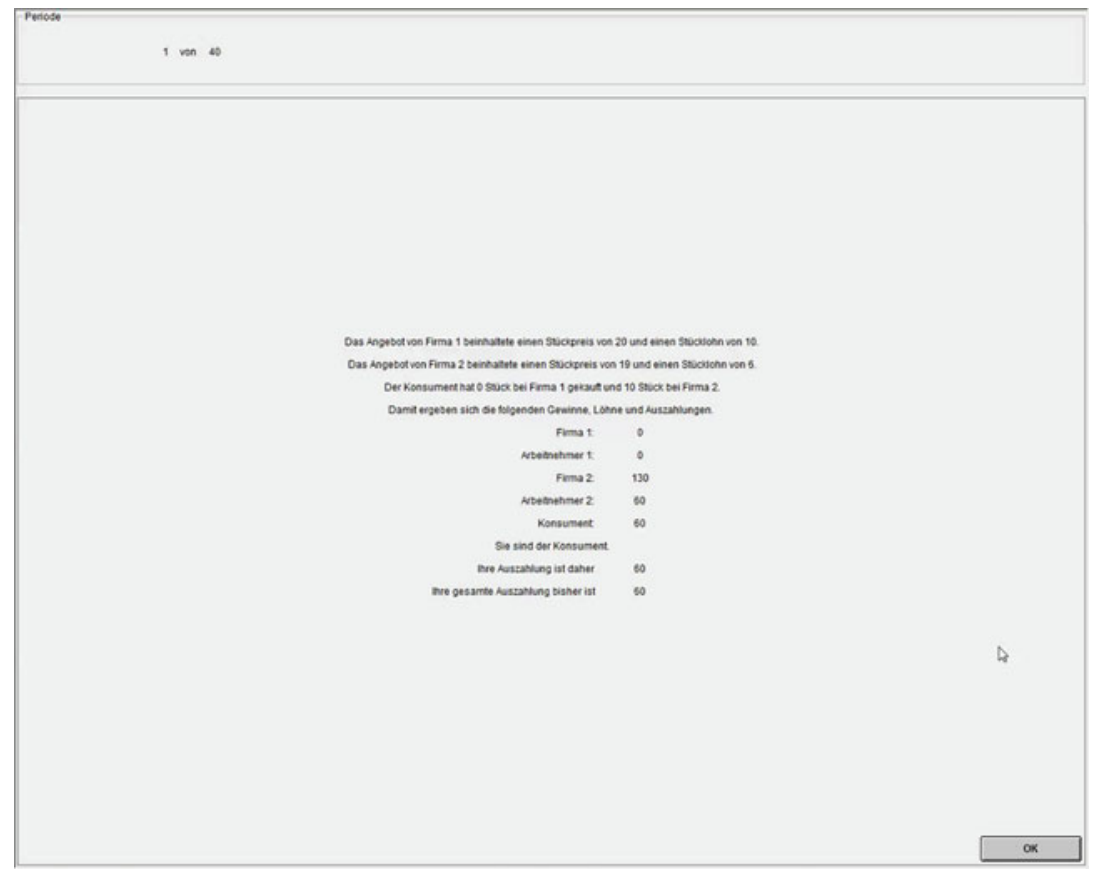

Abb.5.27 Benutzeroberfläche für alle ProbandInnen der Spielgruppe

des Spielmoduls erfolgt hier durch die Programmierung selbst in technischmediatisierter Form. Die ,Spielräume' für die Aktivitäten der ProbandInnen und die Informationen, die ihnen zur Verfügung gestellt werden, sind durch die von ihnen eingenommenen Rollen bestimmt. Das experimentspezifische Spielmodul, welches die Geltung seiner Regeln beansprucht, wird hier durch die technische Vermittlung in die Situation eingeprägt. Durch die technische Vermittlung der Handlungsspielräume wird auf diese Weise das Doing rationaler Verhaltenserwartung gegenüber den ProbandInnen bestärkt. Auf technischer Ebene wird also eine strukturierte Setzung von vorgegebenen Handlungsspielräumen vollzogen, die zugleich das mögliche Spektrum alternativer Handlungen begrenzt. Bei den oben dargestellten Beispielen tritt zugleich die charakteristische Verknüpfung 
zwischen Spiel-, Ökonomie- und Experimentmodul in Erscheinung. Die Entscheidungsabhängigkeit der erspielten Gewinne ${ }^{71}$ wird durch die Sequenzialität der Handlungsschritte und zu bearbeitenden Bildschirmoberflächen deutlich akzentuiert. Handlungen und Wahlentscheidungen, die den technischen Eingabevorgaben entsprechend gemacht werden, erweisen sich als folgenreich und wirken sich auf nachfolgende Situationen aus. Die zeitliche Distanz zwischen den getätigten Eingaben und ihren Konsequenzen ist vom spezifischen Experiment und dem eingesetzten Mechanismus abhängig. Das Induzieren des Rahmens wird hier deutlich verstärkt. Die Möglichkeit dieser Betonung ökonomischer Konsequenzen von Entscheidungen gründet dabei zugleich in der Spezifik der eingesetzten Mediation kommunikativer Handlungen und ihrer Objektivation (siehe Abschnitt 5.3.8.).

So klar der sequentielle Ablauf der Datenerhebung am Computer durch die Software organisiert ist, so klar zeigen die nachfolgenden empirischen Beispiele auf, welchen hohen Stellenwert die Methoden der situativen Handlungskoordination für den geordneten und störungsfreien Ablauf der Laborexperimente besitzen. Mittels performativer Methoden unterstützen die ExperimentatorInnen gezielt den synchronen und sequenziellen Ablauf, der durch die Programmierung der Treatments geplant ist. Der Übersichtsbildschirm der ExperimentatorInnen ist eines der zentralen Tools der anwesenden Labormitglieder und wird in der empirischen Hervorbringung der kommunikativen Konstruktion des Laboratops mit verschiedenen situationsabhängigen Ethnomethoden verknüpft. Es zeigt sich eine spezifische Orchestrierung unterschiedlicher Arten der Vermittlung kommunikativer Handlungen.

\subsubsection{Körperliche Präsenz der ExperimentatorInnen und ihre Nutzung}

$\mathrm{Zu}$ den informellen Regeln des Laborexperiments gehört es, dass die ProbandInnen während der Durchführung des Experiments ihre Arbeitskabinen nicht verlassen dürfen. Den Mitgliedern des Laborteams ist es hingegen erlaubt, sich während dieser Zeit frei im Laborraum zu bewegen. Den ProbandInnen und Labormitgliedern werden im Rahmen des Experiments somit nicht nur unterschiedliche Rederechte (siehe Abschnitt 5.4.2), sondern auch unterschiedliche Bewegungsrechte zuerkannt. Wie im Zuge der Raumstruktur des Labors beschrieben, können die Kabinen der ProbandInnen nur von der Rückseite betreten

\footnotetext{
${ }^{71}$ Die entscheidungsabhängige Entlohnung von ProbandInnen ist, wie dargestellt, gängiger Standard bei Experimenten aus dem Bereich der experimentellen Wirtschaftswissenschaft
} 
werden. Aus dieser Position ist es ebenfalls möglich, in die Arbeitskabine hineinzuschauen, einen Blick auf den Bildschirm der ProbandInnen zu werfen und zu erkennen, mit welcher Benutzeroberfläche sie gerade interagieren. Die Mitglieder des Laborteams nutzen die in ihrer Rolle begründete Bewegungsfreiheit nicht nur, um die visuellen Beschränkungen der Trennwände zu überwinden, sondern setzen ihre körperliche Bewegung im Raum wie auch ihre körperliche Präsenz gezielt in unterschiedlichen typischen Ethnomethoden ein. Die performative Wirkung dieser kommunikativen Handlungen besitzt eine wichtige Bedeutung für die kommunikative Konstruktion des Laboratops. Wie nachfolgend aufgezeigt, hat der Rundgang durch das Labor und die Veränderung der körperlichen Präsenz der Mitglieder des Laborteams eine performative Wirkung. In unterschiedlichen Situationen wird dies gezielt im Labor eingesetzt, um die Wirkung anderer Methoden zu ergänzen, zu verstärken oder zu überprüfen.

Die praktische Methode des Rundgangs ist ein Beispiel für eine typische Handlung der ExperimentatorInnen. Ein Mitglied des Laborteams verlässt dazu den Arbeitsplatz der ExperimentatorInnen und läuft den Gang zwischen den Arbeitsplätzen entlang, folgte diesem auf der anderen Seite des Raumes bis er bzw. sie wieder am Arbeitsplatz der ExperimentatorInnen angekommen ist. Während ein Mitglied des Laborteams die Gänge des Labors entlanggeht, wirft es einen kurzen Blick auf die Bildschirme der einzelnen ProbandInnencomputer, an denen es vorbeigeht.

Eine typische Phase, in der diese Handlung vollzogen wird, ist kurz nachdem die ProbandInnen begonnen haben, die Aufgabenstellungen am Computer zu bearbeiten. Auf ihrem Weg kontrollieren die ExperimentatorInnen hier durch einen kurzen Blick auf die Bildschirme die ordnungsgemäße Funktion des Programms an den Computern der ProbandInnen (Abb. 5.28).

Eine alternative oder ergänzende Methode, um zu kontrollieren, ob die Nutzerapplikationen an allen Arbeitsplätzen der ProbandInnen ordnungsgemäß funktionieren, ist die verbale Nachfrage eines Mitglieds des Laborteams. Diese besteht darin zu erfragen, ob alle im Labor befindlichen Personen den grauen Startbildschirm des Programms z-leaf sehen. Dies erfolgt stets, nachdem die ExperimentatorInnen den Handlungskomplex der Nachfragen zu den Instruktionen $^{72}$ für abgeschlossen erklärt haben. Sollte an einem Arbeitsplatz das Programm nicht wie vorgesehen funktionieren, so werden die ProbandInnen gebeten, dies per Handzeichen zu signalisieren. In den beobachten Experimenten wurden häufig beide Kontrolltechniken nacheinander angewandt.

\footnotetext{
${ }^{72}$ Dies entspricht dem Gliederungspunkt ,3. Zeit für die Beantwortung von Nachfragen der Teilnehmenden“" (siehe Abschnitt 5.3.1)
} 


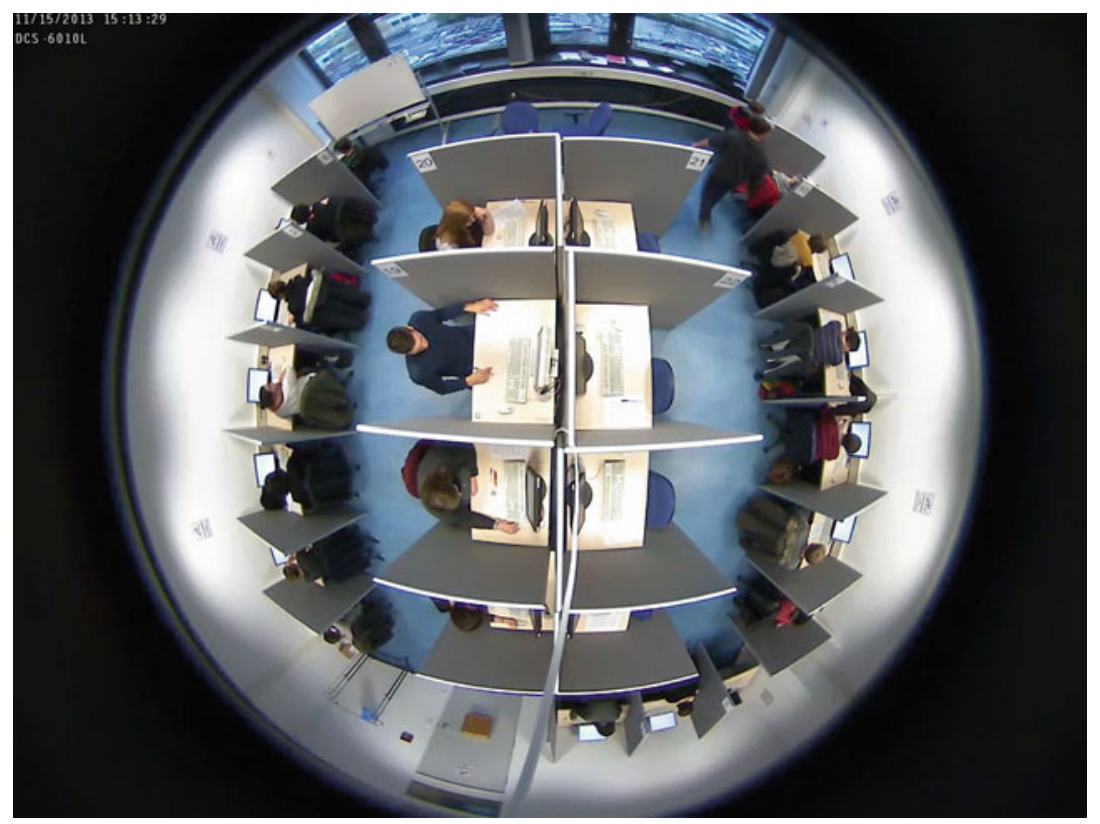

Abb.5.28 Visuelle Kontrolle des Experimentatoren

Auch während die ProbandInnen am Computer ihre Aufgaben erfüllen, vollziehen die ExperimentatorInnen in regelmäßigen Abständen einen Rundgang durch das Labor. Im Unterschied $\mathrm{zu}$ den ProbandInnen ist es den Mitgliedern des Labors gestattet, sich im Raum zu bewegen. Tun sie dies, so wird für die ProbandInnen offenbar, dass die visuelle Abschirmung ihrer Arbeitskabine sie nur solange vor den Blicken anderer anwesender Personen schützt und die Anonymität ihrer Handlungen wahrt, solange sich niemand hinter ihrem Rücken befindet. ${ }^{73}$

\footnotetext{
${ }^{73}$ Wie im Methodenkapitel ausgeführt, versuchte ich in einer frühen Phase der Beobachtung der Abläufe im Labor ebenfalls die Handlungen der ProbandInnen an ihren Arbeitsplätzen direkt zu beobachten. Aufgrund der visuellen Beschränkungen durch die Trennwände wählte ich dafür eine Position an der offenen Rückseite der Arbeitskabinen. Ich stand somit hinter dem Rücken des jeweiligen Teilnehmenden. Es zeigte sich von Seiten der ProbandInnen eine starke Reaktanz. Sie waren sichtlich verunsichert. Sie drehten sich wiederholt zu mir um und nahmen vermehrt eine steifere Sitzposition ein. Teilweise sprachen sie mich auch an, um nachzufragen, ob sie einen Fehler gemacht hätten. Die Situation und auch die ProbandInnen entspannten sich erst wieder, nachdem ich meine Beobachtungsposition verlassen
} 
Ob die ExperimentatorInnen die einzelnen ProbandInnen tatsächlich beobachten, während sie an den Arbeitskabinen vorbeigehen, ist für die ProbandInnen nicht ersichtlich, da die ExperimentatorInnen hinter ihnen vorbeigehen. Da es außer den Klickgeräuschen von Tastatur- und Mauseingaben jedoch sonst keine weiteren Geräusche im Laborraum gibt, können die ProbandInnen das Laufen des Labormitglieds akustisch und unter Umständen auch durch ihr peripheres Sehen wahrnehmen. Die ProbandInnen können so durchaus ausmachen, wann sich die ExperimentatorInnen ihrem Arbeitsplatz nähern und diesen passieren. Der Rundgang ist somit eine Kontrolltechnik, mit der sowohl das geplante Funktionieren der Technik als auch das sichtbare Verhalten der ProbandInnen überwacht werden kann.

Für die interaktive Koordination der Handlungsabläufe im Labor hat der Rundgang jedoch nicht nur diese Kontrollfunktion des regelkonformen Handelns der ProbandInnen. Mit dem Rundgang geht eine deutliche Verringerung der räumlichen Distanz zwischen den Labormitgliedern und den einzelnen ProbandInnen einher. Wie das Beispiel der Meldung der Probandin Katja zeigte ${ }^{74}$, kann die visuelle Abschirmung durch die Trennwände durchaus zu einer Herausforderung für das Stellen einer regelkonformen Nachfrage werden. Der Rundgang der Labormitglieder bildet zugleich ein ,interaktives Angebot' an die ProbandInnen, um Nachfragen zu stellen und die kurzfristige Verringerung der räumlichen Distanz so situativ zu nutzen. In den beobachteten Experimenten wurde das interaktive Angebot des Rundgangs in dieser Funktion auch intensiv von den ProbandInnen genutzt. Typischerweise wenden sich die VersuchsteilnehmerInnen der Laufrichtung der ExperimentatorIn entgegen, versuchen Blickkontakt mit diesen aufzunehmen und heben kurz vor dem Vorbeigehen des Labormitglieds ihre Hand. Tritt das Mitglied des Laborteams die wenigen verbleibenden Schritte an den Arbeitsplatz des Teilnehmenden heran, so beginnt die Frage-Antwort-Interaktion. Das Standbild in Abbildung 5.29 illustriert eben diesen Aspekt. Der Proband Marc, welcher am Arbeitsplatz 20 sitzt, nimmt hier Blickkontakt mit dem Experimentator Tim auf, um eine Frage-Antwort-Interaktion zu initiieren. Kurz bevor Tim den Arbeitsplatz des Probanden Marc erreicht hat, hebt dieser die Hand. Die erfolgreiche Imitierung der Frage-Antwort-Interaktion gelingt. Der Experimentator Tim tritt an die Kabine des Probanden heran, welcher wiederum seine Frage

hatte. Erst durch mein eigenes Erlernen des Einsatzes körperlicher Präsenz als Ethnomethode der ExperimentatorInnen wurde mir deutlich, warum die direkte Beobachtung keine gegenstandsadäquate Erhebungsmethode im Labor war.

${ }^{74}$ Siehe dazu Abschnitt 5.4.3. 
stellen kann. Wie die Abbildung ausweist, ist Marc die einzige Versuchsperson, deren Blickrichtung nicht auf das Innere der Kabine gerichtet ist.

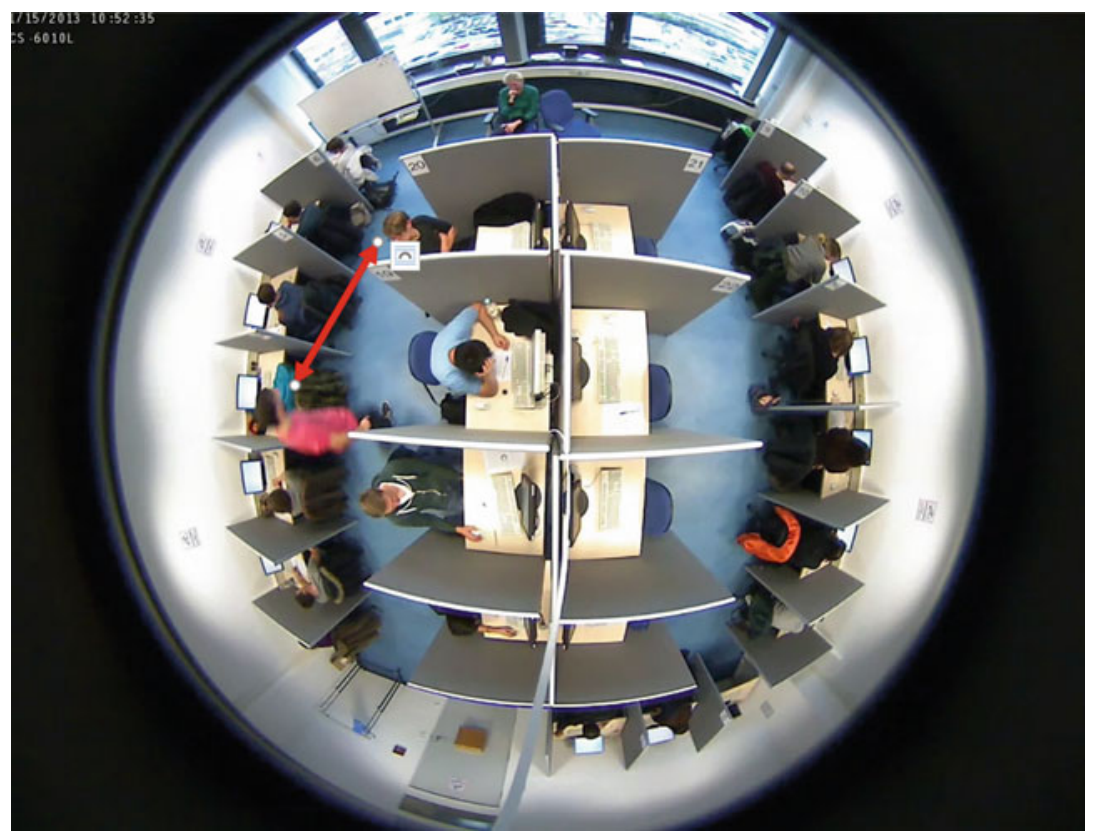

Abb.5.29 Initierung einer Frage-Antwort-Interaktion

Die Musterhaftigkeit, welche diese Handlung der ProbandInnen in der Durchführung von Experimenten zeigt, legt es nahe, dass sie eine Ethnomethode der ProbandInnen ist. Diese bildet ebenfalls ein Element des Doings der Verhaltenserwartungen. Es zeigt sich hier die interaktive Hervorbringung und Anwendung des Spielmoduls im Labor. Das Accounting der Verhaltenserwartungen an die ProbandInnen und ExperimentatorInnen zeigt sich in ihrer anschlussfähigen und situationsangemessenen Nutzung durch die Handelnden selbst. Die Verhaltensregeln im Labor geben vor, dass das laute Stellen von Fragen untersagt ist und dass zur Initiierung von Frage-Antwort-Interaktionen Handzeichen gegeben werden sollen. Es wird jedoch nicht bestimmt, wohin die ProbandInnen innerhalb ihrer Kabine schauen dürfen. Wie oben dargestellt, wird die Körperpositionierung der ProbandInnen während des Agierens mit den Bildschirmoberflächen und 
Eingabegeräten durch die materielle Einrichtung der Arbeitsplätze auf das Innere der Kabinen ausgerichtet. Nehmen die ProbandInnen diese Position ein, so wirken die Trennwände als Objektivationen der visuellen Abschirmungsbemühungen. Die Regeln des Spiels anerkennend, nutzen die ProbandInnen den nicht offiziell bestimmten Freiraum auf typische Weise.

Der Rundgang dient primär der visuellen Kontrolle der Arbeitsplätze und abläufe der ProbandInnen. Er überwindet dabei die visuellen Beschränkungen durch die Trennwände, die es auch den ExperimentatorInnen unmöglich machen, die Arbeitsplätze der ProbandInnen einzusehen. Die Vereinzelung der ProbandInnen in den Arbeitskabinen und die zugesicherte Anonymität der Handlungen und Entscheidungen basiert stark auf der visuellen Abschirmung und dem Gefühl der ProbandInnen, unbeobachtet zu sein. Der Rundgang der ExperimentatorInnen zeigt für die ProbandInnen auch auf, dass diese in der situativen Ordnung des Labors über Bewegungsrechte verfügen, die es ermöglichen, die visuelle Abschirmung auszusetzen und in die Kabinen der ProbandInnen visuell einzudringen. Analog zur Machtasymmetrie, die sich hinsichtlich der Kommunikationsrechte zwischen ProbandInnen und ExperimentatorInnen ergibt, liegt diese auch für die Bewegungsrechte vor als Momente des Spielmoduls, die mit diesen Rollen verbunden sind.

Beim Rundgang handelt sich um eine Ethnomethode, die einerseits das Doing einer spezifischen Laborsituation unterstützt und dazu verhilft, den ordnungsgemäßen und geplanten Ablauf der Sessions zu kontrollieren. Von Seiten der ProbandInnen wird die geringere körperliche Distanz, die mit dem Passieren der einzelnen Arbeitsplätze einhergeht, jedoch auch als typische Chance genutzt, um Nachfrageinteraktionen zu initiieren. Die Inanspruchnahme und der performative Einsatz dieser Bewegungsrechte der ExperimentatorInnen verankern hier diesen Teil des Spielmoduls. Gerade in Kombination mit anderen musterhaften kommunikativen Handlungen wird diese Technik auch vielfach eingesetzt, um das Doing der rationalen und regelkonformen Verhaltenserwartungen zu befördern.

\subsubsection{Die situative Orchestrierung von unterschiedlichen kommunikativen Modalitäten}

In der Durchführung von Experimenten treten die typischen Muster kommunikativen Handelns meist nicht getrennt voneinander auf. Vielmehr werden diese häufig gezielt von den ExperimentatorInnen miteinander verwoben. Anhand verschiedener typischer Beispiele wird nachfolgend aufgezeigt, wie die Labormitglieder die gekonnte Orchestrierung unterschiedlicher technischer, verbaler und körperlicher 
Techniken musterhaften kommunikativen Handelns anwenden, um die kommunikative Konstruktion des ökonomischen Laboratops situativ zu realisieren und die angestrebte situative Ordnung im Labor zu (re-)produzieren. Damit bilden sie wichtige Elemente in der Verankerung des Rahmens.

Wie weiter oben beschrieben ermöglicht der Übersichtsbildschirm den ExperimentatorInnen, den Arbeits- bzw. Bearbeitungsfortschritt der ProbandInnen an ihren Arbeitsplätzen zu überwachen (siehe Abschnitt 5.4.4). Die auf der „Clients' Table" dargestellten Informationen stellen Objektivationen kommunikativer Handlungen der aktuellen Bearbeitungsphasen der einzelnen ProbandInnen dar. Eine zentrale Handlungskompetenz der ExperimentatorInnen besteht darin, auf Basis dieser kommunikativen Objektivationen situativ zu entscheiden, wann sie eingreifen sollten, um den Vollzug der Bearbeitung der gestellten Aufgaben zu animieren. Dafür ist es von entscheidendem Vorteil, wenn die ExperimentatorInnen die aktiven Benutzeroberflächen kennen, die den ProbandInnen angezeigt werden. Alternativ kann auch die in der Programmierung hinterlegte Benennung der Benutzeroberflächen Auskunft darüber geben, welche Handlung von den ProbandInnen vollzogen werden muss. Im oben dargestellten Fall einer "Clients' Table“ (Abb. 5.20) wurden beispielsweise die Benutzeroberflächen „Offer period<max_period“" und „Offer Auswertung < max_period“ abgebildet. Die durchführenden ExperimentatorInnen wussten in diesem Fall, dass einige der ProbandInnen damit befasst waren, ein Gebot einzugeben (,Offer period<max_period"), andere diesen Schritt bereits vollzogen hatten und ihnen bereits das Ergebnis ihrer Handlung des Gebotssetzens angezeigt wurde („Offer Auswertung < max period"), welches sie nachfolgend durch das Drücken des OK-Buttons bestätigen mussten.

In Situationen, in denen sich eine deutliche Differenz zwischen den Bearbeitungsphasen der ProbandInnen zeigte, war vielfach eine typische kommunikative und in diesem Fall zugleich gezielt performative Handlung von Labormitgliedern beobachtbar. Die Kommunikations- und Bewegungsrechte, über welche die ExperimentatorInnen verfügen, wurden hier situativ angemessen eingesetzt, um unterschiedliche Muster kommunikativen Handelns zu realisieren. Je nach Bearbeitungsphase und damit verbundener Benutzeroberfläche werden von den Labormitgliedern gezielt verschiedene Methoden eingesetzt, um die Verhaltenserwartungen gegenüber den ProbandInnen zu kommunizieren und die Ausführung der von ihnen geforderten Handlungen zu beschleunigen.

Handelt es sich um Benutzeroberflächen, die die Bestätigung von Eingaben oder angezeigten Ergebnissen beinhalten, wie beispielsweise „Offer Auswertung<max_period“", wird in einem ersten Schritt durch eine verbale Äußerung versucht, die ProbandInnen daran zu erinnern, dass sie durch ein Klicken 
auf die entsprechende Schaltfläche die Kenntnisnahme der angezeigten Ergebnisse oder Informationen bestätigen müssen. Eine typische Phrase, mittels derer dies bewerkstelligt wird, ist: „Bitte vergessen Sie nicht auf den OK-Button zu drücken“. In dieser Sprechsituation rahmen die ExperimentatorInnen ihre Aussage als eine allgemeine Information. Sie verweisen nicht darauf, dass sie durch die Objektivation(en) der „Clients' Table“ Kenntnis darüber haben, dass einige ProbandInnen diesen Schritt bereits vollzogen haben. ${ }^{75}$ Mittels einer so gearteten performativen Äußerung soll erreicht werden, dass alle ProbandInnen angezeigte Ergebnisse oder Informationen zeitnah bestätigen, nachdem sie die entsprechenden Informationen durchgelesen haben.

Ein anderer Fall liegt vor, wenn eine Handlung vollzogen werden muss, in der eine aktive Eingabe von den ProbandInnen gefordert ist. Um den Zeitpunkt für den Einsatz performativer Handlungen zu bestimmen, greifen die ExperimentatorInnen auch hier auf ihre Erfahrungswerte hinsichtlich üblicher Bearbeitungszeiten zurück. Für das Lesen und Bestätigen von Informationen benötigen die ProbandInnen in der Regel deutlich kürzer, als für das Einsetzen von Zahlenwerten (wie Geboten oder Preisen). Wenn den ExperimentatorInnen durch den Blick auf den Übersichtsbildschirm der „Clients” Table“ deutlich wird, dass eine auffällig große Anzahl der ProbandInnen eine Handlungsphase abgeschlossen hat und sie nun auf einzelne ProbandInnen warten müssen, welche noch mit dem Handlungsschritt befasst sind, vollziehen die ExperimentatorInnen häufig einen, gezielten Rundgang' (zum Rundgang siehe auch Abschnitt 5.4.6).

Auch hier zeigt sich eine übliche Kombination von unterschiedlichen praktischen Methoden. Technische Objektivationen werden mit performativen Handlungen der ExperimentatorInnen verknüpft. Die Ethnomethode des Labormitglieds besteht hier darin, im Zuge eines Rundgangs durch das Labor die Bildschirmoberflächen zu erfassen, welche den einzelnen ProbandInnen angezeigt werden. Zwischen der Benutzeroberfläche, welche die ProbandInnen zum Warten auffordert, und derjenigen, die eine Eingabe oder Aktion erfordert, besteht eine optische Differenz. Durch den Rundgang können die ExperimentatorInnen überprüfen, ob die ProbandInnen, die den Arbeitsschritt noch nicht abgeschlossen haben, mit

\footnotetext{
${ }^{75}$ Diese Vagheit der Formulierung ist auch dadurch begründet, dass den ProbandInnen bei der Anzeige von Ergebnissen eine ausreichend lange Zeit gegeben werden soll, um diese zu betrachten. Die gezielte Präsentation von Informationen soll die Grundlage für weitere Entscheidungen und Handlungen der ProbandInnen schaffen. Wie die Ausführungen zur Methodologie der Experimente und den paradigmatischen Annahmen der experimentellen Wirtschaftswissenschaftler zeigten, ist gezielte Informationskontrolle ein wichtiges Kriterium in diesen Experimenten. Wie viel Zeit einzelne ProbandInnen benötigen, um die für sie relevanten Informationen zu erfassen, ist dabei unterschiedlich.
} 
ebendiesem noch befasst sind oder lediglich vergessen haben, die Eingabe zu bestätigen. Diese Vermutung liegt für die ExperimentatorInnen nahe, wenn bereits Zahlenwerte eingetragen oder Auswahlmöglichkeiten ausgewählt sind.

Das Mitglied des Laborteams vollzieht diese Überprüfung häufig in einem ungleichmäßigen Lauftempo. Sie verlangsamen bei den Arbeitskabinen, die sie durch die differente Benutzeroberfläche selektiert haben. In solchen Fällen, in denen die Gruppe nur noch auf einzelne ProbandInnen wartet, kreuzen die ExperimentatorInnen auch häufiger den Arbeitsplatz oder verharren für einige Momente am Arbeitsplatz einer Versuchsperson.

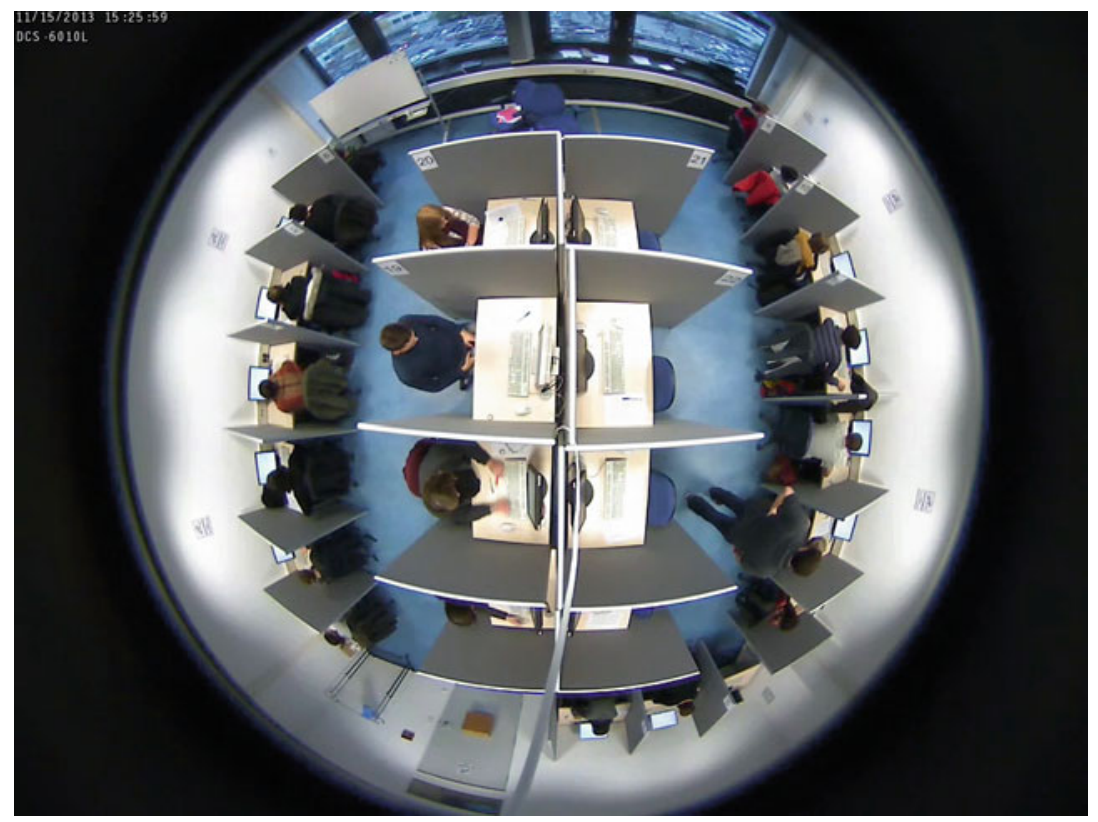

Abb.5.30 visuelle Überprüfung des Experimentatoren

Experimentator Tim tritt im abgebildeten Screenshot (Abb. 5.30) an den Arbeitsplatz 13 heran, um eben diese Überprüfung zu vollziehen. Aus oben 
genannten Gründen der performativen Wirkung körperlicher Präsenz ${ }^{76}$ beschleunigt die Anwesenheit eines Labormitglieds in vielen Fällen den Handlungsprozess der ProbandInnen.

Wie oben beschrieben wird der Rundgang auch genutzt, um Nachfrageinteraktionen zu initiieren. Dies gilt sowohl für die ProbandInnen als auch für die ExperimentatorInnen. Im Unterschied zu den ProbandInnen stellt das Initiieren von Nachfragen durch das „zufällige Vorbeilaufen“ eines Labormitglieds zugleich eine Technik der selbigen dar, um auffällige ProbandInnen zu schnellerem Agieren im Experiment anzuregen oder Probleme aufzudecken. Zeigt sich über mehrere Runden hinweg, dass es wiederholt die gleichen ProbandInnen sind, die auffällig mehr Zeit für die Bearbeitung benötigen, erhöht die ExperimentatorIn die Intensität ihres Eingriffs und tritt dichter an den Arbeitsplatz des entsprechenden Teilnehmenden heran. Unter Rückgriff auf die Kommunikationsrechte, über die ExperimentatorInnen im Vollzug des Experiments verfügen, wird mittels einer freundlichen Ansprache bei den VersuchsteilnehmerInnen nachgefragt, ob es Fragen oder Probleme bei der Bearbeitung der gestellten Aufgabe gibt.

Im Abschnitt über die technische Realisierung des sequenziellen Ablaufs (Abschnitt 5.4.5) wurde dargestellt, wie durch den Einsatz der Software der sequenzielle Ablauf einzelner Phasen des Experiments koordiniert wird. Die Software wird jedoch auch dazu genutzt, um zwischen unterschiedlichen Modalitäten der Kommunikation zu vermitteln. In einer Vielzahl der beobachteten Experimente gab es Phasen, in denen die ProbandInnen nicht nur Eingaben am Computer machten, sondern auch zu anderen Aktivitäten aufgefordert wurden. In einigen Fällen wurden Handlungen im Labor vollzogen, die sich die ProbandInnen anschauen sollten oder sie wurden gebeten, einzelne Abschnitte der Instruktionen erneut durchzulesen. Mittels der Benutzeroberfläche wird hier eine Vermittlung zwischen Handlungen im Labor und Handlungen der ProbandInnen an ihren Eingabegeräten koordiniert und synchronisiert. Dies erfolgt in Kombination mit den kommunikativen Handlungen und Ethnomethoden der ExperimentatorInnen, welche auf den Kommunikationsrechten ihrer sozialen Rolle fußen (Abschnitt 5.4.2). Die kommunikativen Handlungen, die die ExperimentatorInnen im Labor vollziehen, sind in ihrem Ablauf an der im Programm hinterlegten Sequenzstruktur orientiert. Sie bestärken, verdeutlichen oder vermitteln zwischen den auf den Benutzeroberflächen objektivierten Inhalten und den Geschehnissen im Laborraum selbst.

\footnotetext{
${ }^{76}$ Siehe hierzu Abschnitt 5.4.6. Der Rundgang der ExperimentatorInnen macht für die ProbandInnen erfahrbar, dass die Anonymität ihrer Entscheidungen und Handlungen nur gegeben ist, solange niemand hinter ihrer Arbeitskabine steht.
} 
Das nachfolgende Beispiel zeigt auf, wie die Programmierung den Wechsel des Aufmerksamkeitsfokus der ProbandInnen lenkt und wie im Labor zwischen Handlungssequenzen mit unterschiedlichen Modalitäten der Kommunikation strukturiert vermittelt wird. Im Rahmen einiger Treatmentvariationen des Experimentserie „Endowment-Effekt-USB-Stick“ wurden die ProbandInnen aufgefordert, über mehrere Runden ein Spiel zu spielen. Immer wurde der Wechsel zwischen den Handlungsphasen durch das Softwareprogramm und aktive Benutzeroberflächen induziert und gesteuert und zugleich durch kommunikative Handlungen der Labormitglieder situativ gerahmt. Das nachfolgende Bespiel entstammt der Durchführung eines Experiments, in dem mittels eines Wurfs auf einem Rouletterad ein Gewinner und ein Verlierer in einer Zweiergruppe von füreinander anonymen ProbandInnen bestimmt werden sollte. In den schriftlichen Instruktionen wurden die ProbandInnen über die spezifischen Regeln dieses Spiels informiert. Um die visuelle Beschränkung der ProbandInnen in den Kabinen zu reduzieren, wurde der Roulettewurf vor Ort gefilmt und mittels eines Beamers auf einer großen Leinwand im Labor gezeigt. Zu Beginn wurde ein Probedurchgang durchgeführt und im Anschluss der Durchgang, der für die spätere Rollenzuweisung relevant war. Da die technisch realisierte Vermittlungsbeziehung in beiden Durchgängen gleich ist, wird im Folgenden nur der Ablauf des Probedurchgangs betrachtet.

In einem ersten Schritt werden die Handlungen der ProbandInnen synchronisiert (Abb. 5.31). Haben alle ProbandInnen durch das Klicken auf den OK-Button bestätigt, dass sie bereit sind, den Versuch zu sehen, so kann ein Labormitglied dazu übergehen, den Probedurchgang zu beginnen. Durch die Kontrolle der „Clients' Table“ wird von den ExperimentatorInnen bestimmt, wann dieser Zeitpunkt vorliegt und zum nächsten Schritt übergegangen werden kann.

Alle ProbandInnen sehen nun eine Benutzeroberfläche (Abb. 5.32), auf welcher sie aufgefordert werden, ihren Einsatz zu platzieren und eine Zahl zwischen 0 und 36 einzugeben. Ihre Eingabe sollen sie anschließend mittels eines Klicks auf den OK-Button bestätigen. Zeitgleich zur Anzeige der Benutzeroberfläche wird diese Aufgabe durch ein Mitglied des Laborteams zudem noch einmal sprachlich und für alle Anwesenden hörbar kommuniziert. In den ersten beiden Handlungsschritten ist die Aufmerksamkeit der ProbandInnen dabei auf die Benutzeroberfläche gelenkt. Textlich präsentierte Informationen und die strukturierten Eingabe- und Handlungsmöglichkeiten prägen die Vermittlung der kommunikativen Handlungen.

Auf der folgenden Benutzeroberfläche (Abb. 5.33) werden die bisherigen Informationen für die ProbandInnen zusammengestellt. Sowohl die von ihnen gewählte Zahl als auch die ihres „Gegenspielers“ werden ihnen präsentiert. Die Teilnehmenden werden zudem darüber informiert, dass die ExperimentatorIn das 


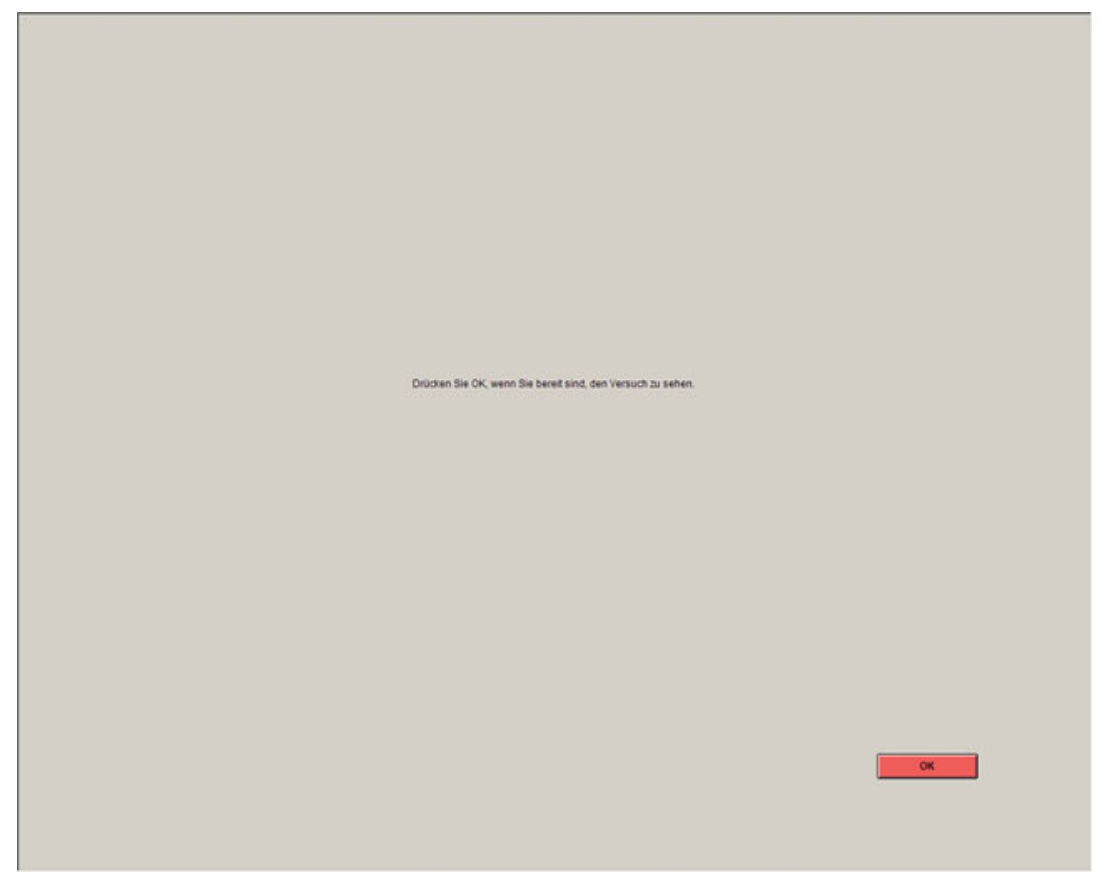

Abb.5.31 Aktiver Screen zur Synchronisierung des Probedurchlaufs

Rouletterad drehen und die Gewinnzahl bestimmen wird. Diese Information wird von einem Labormitglied für alle hörbar wiederholt und initiiert damit einen Wechsel des Aufmerksamkeitsfokus der ProbandInnen. Statt auf ihren Bildschirm zu blicken, sollen sie nun zur ExperimentatorIn bzw. auf die Leinwand schauen, auf der der Versuch gezeigt wird. Mit der Vermittlung kommunikativer Inhalte und dem Übergang zu einer anderen Modalität der Kommunikation geht hier auch in räumlicher Hinsicht eine Änderung der kommunikativen Situation einher. Es erfolgt ein Wechsel von eine multi-zentrierten $\mathrm{zu}$ einer fokussierten Interaktionssituation. Die Vereinzelung der ProbandInnen in ihren Arbeitskabinen wird für diesen Handlungsabschnitt ausgesetzt. Alle ProbandInnen sollen nun wieder gemeinsam als Gruppe nach vorne schauen und die Handlungen des Labormitglieds verfolgen. Haben alle ProbandInnen bestätigt, dass sie die Informationen zur Kenntnis genommen haben, kann zum Roulettewurf übergangen werden. Um 

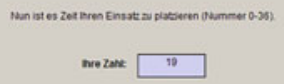

Abb.5.32 Aktive Benutzeroberfläche - Roulettespiel - Zahl eingeben

den Zeitpunkt zu bestimmen, an dem zum nächsten Abschnitt der größeren Handlungssequenz übergegangen werden kann, müssen die ExperimentatorInnen auch hier auf den Übersichtsbildschirm und spezifischer auf die Spalte ,state“ der „Clients' Table“ (Abb. 5.20) des z-Tree-Programms achten. Die praktische Initiierung neuer Handlungsabschnitte ist somit eine Ethnomethode, die an die Kompetenzen der situativen Handlungskoordinierung der Labormitglieder gebunden ist. Die Umsetzung dieser Ethnomethode der ExperimentatorInnen ist jedoch zugleich von den technischen Voraussetzungen abhängig, welche binnenstrukturell und damit situationsunabhängig vorliegen. ${ }^{77}$

\footnotetext{
${ }^{77}$ Diese Programmierung der Treatmentvariation wurde in allen durchgeführten Sessions eingesetzt. Das Ablaufschema der Vermittlung zwischen den Handlungen der ExperimentatorInnen und den Handlungen der ProbandInnen wurde in dieser Phase der Durchführung des Experiments somit in standardisierter und gleichartiger Weise festgelegt.
} 


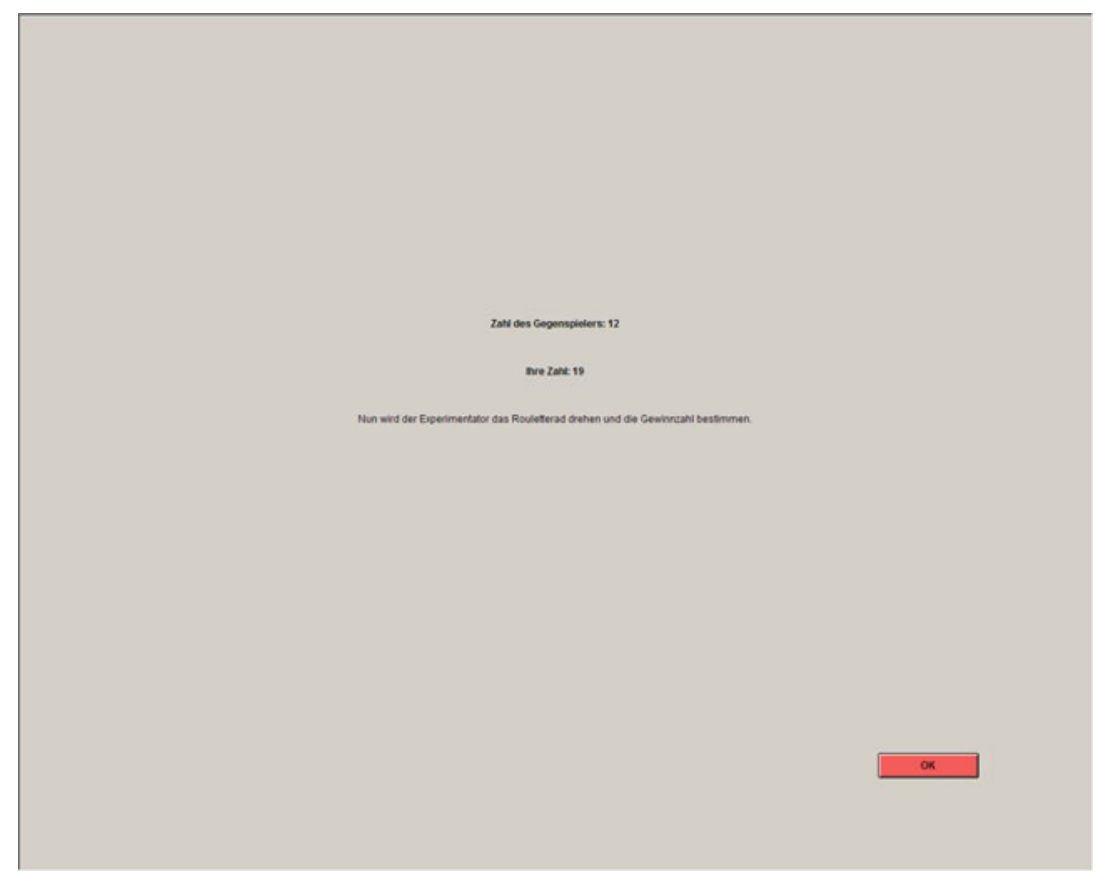

Abb.5.33 Aktive Benutzeroberfläche-Hinweis auf bevorstehenden Roulettewurf (Wechsel des Aufmerksamkeitsfokus)

Das Mitglied des Laborteams vollzieht nun den Roulettewurf. Die ProbandInnen im Labor können dies simultan auf der Leinwand beobachten. Hat die Roulettekugel eine feste Position eingenommen und das Rouletterad seine Geschwindigkeit verringert, so wird das Ergebnis durch die durchführende ExperimentatorIn bekanntgegeben. Die ProbandInnen werden im Folgenden aufgefordert, das soeben beobachtete Ergebnis am Computer in das vorgesehene Feld einzutragen (Abb. 5.34). Die Aufmerksamkeit der ProbandInnen wird hier, vermittelt durch die kommunikativen Handlungen des Mitglieds des Laborteams, wieder auf den Computerbildschirm und die Benutzeroberfläche gelenkt. Der Roulettewurf der ExperimentatorIn, welchen die ProbandInnen gesehenen und gemeinsam erlebt haben, wird nach den Vorgaben der Eingabemaske der Benutzeroberfläche objektiviert. Er wird in eine Zahl überführt, die das Ergebnis des Wurfs wiedergibt und in die Maske eingetragen wird. Der Übergang zur nächsten aktiven 
Benutzeroberfläche wird nicht mehr durch die ExperimentatorInnen bestimmt, sondern durch das Ablaufschema der Programmierung induziert. Er erfolgt, wenn beide ProbandInnen einer Zweiergruppe die gleiche Eintragung hinsichtlich des Ergebnisses des Roulettewurfs gemacht und dieses durch das Klicken auf den OK-Button bestätigt haben.

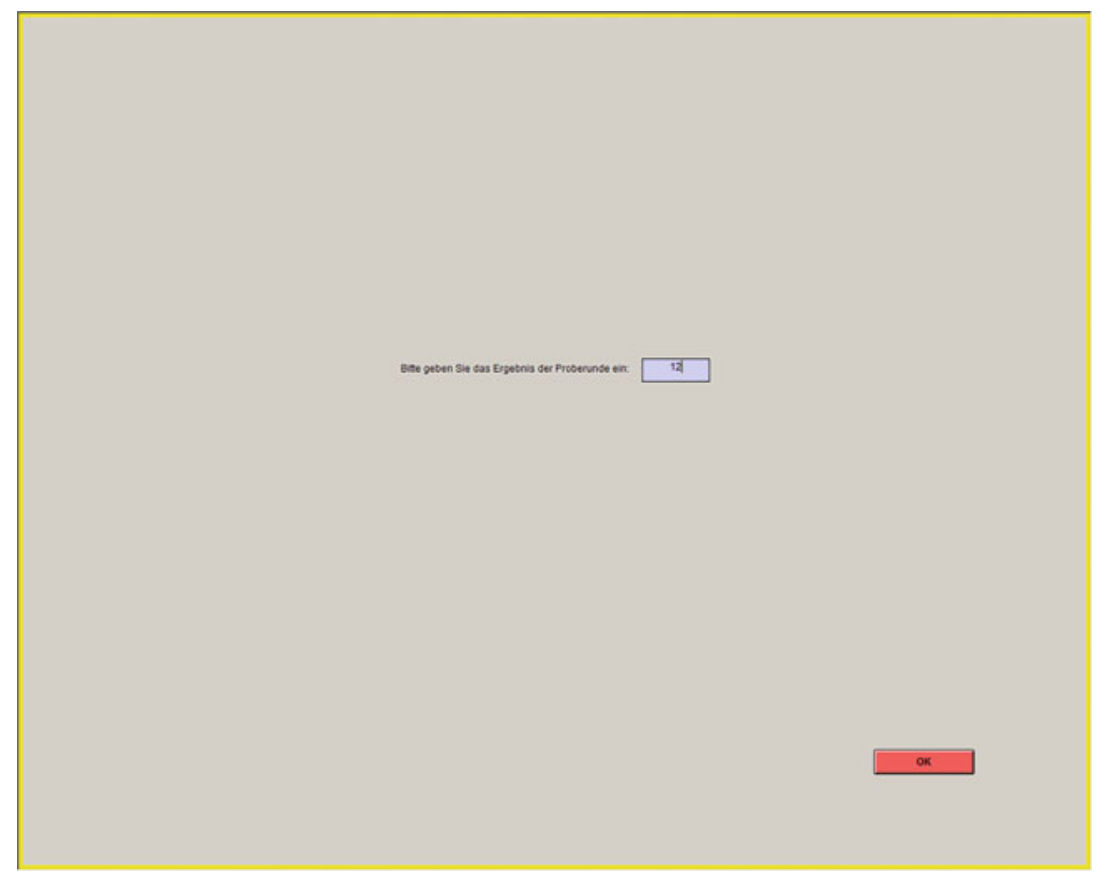

Abb. 5.34 Ergebnis des Roulettewurfs eintragen (Wechsel des Aufmerksamkeitsfokus)

Nach diesem Schritt wird den ProbandInnen das jeweilige Ergebnis ihrer Zweiergruppe angezeigt. Das eingetragene Ergebnis des Roulettewurfs bildet nun die Grundlage, um zu bestimmen, wer von den beiden ProbandInnen das Spiel gewonnen und verloren hat. Im Beispielfall hatte die Person der Zweiergruppe gewonnen, die mit ihrer eingetragenen Zahl dichter an der Zahl des Roulettewurfs lag. Die Entfernung wurde durch die Position der Zahlen auf dem Rouletterad bestimmt und nicht nummerisch. Im nachfolgend abgebildeten Screenshot 
(Abb. 5.35) lag die Zahl des ,Gegenspielers‘ dichter an der Gewinnzahl. Die VersuchsteilnehmerIn musste im Folgenden eine von zwei Lotterien auswählen, die dann am Ende des Experiments ausgespielt wurde.

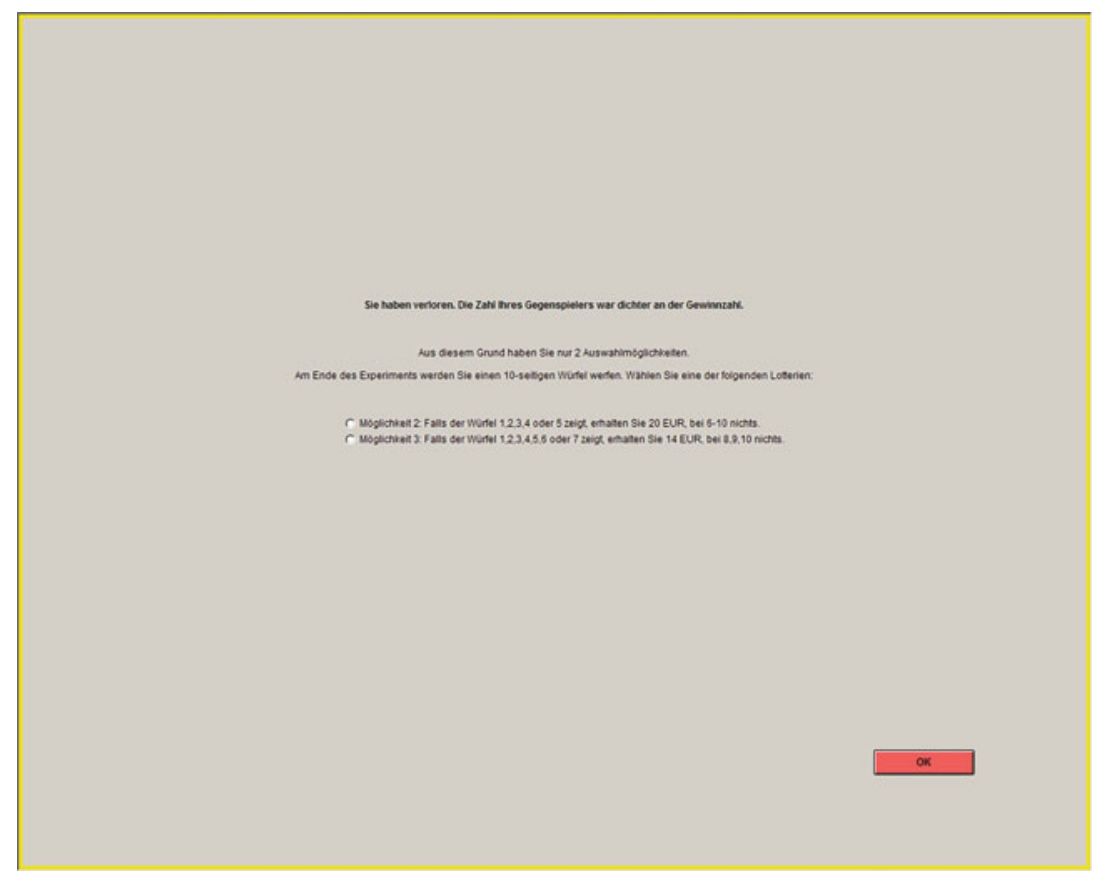

Abb.5.35 Ergebnispräsentation und Lotteriewahl

Die ProbandInnen wurden darüber informiert, dass das Ergebnis der Lotterie am Ende des Experiments von ihnen selbst durch den Wurf eines 10-seitigen Würfel bestimmt wird. Es wurde den ProbandInnen somit die Konsequenz ihrer Handlungen und Wahlentscheidungen aufgezeigt und zugleich auch, wie ihre Gewinnmöglichkeit durch ihre eigenen Entscheidungen geprägt werden.

Zwischen der Auswahl einer Lotterie und dem Vollzug des Würfelwurfs bestand die Aufgabe der ProbandInnen darin, einen Fragebogen zum Thema „Spielen und Exklusivität“ auszufüllen. Am Ende des Experiments, nachdem der Würfelwurf vollzogen wurde, musste das Ergebnis in ein vorgesehenes Bildschirmfeld eintragen werden. Das Ergebnis dieser Eintragung wird im Folgenden 
wiederum auf einer Bildschirmoberfläche angezeigt, um den erspielten Gewinn für das Programm und die Datenerfassung nachvollziehbar zu bestimmen. Auch in dieser Handlungsphase erfolgte somit abermals ein Wechsel zwischen unterschiedlichen kommunikativen Modalitäten, der durch die Programmierung und die Handlungen der ExperimentatorInnen und ProbandInnen vermittelt wurde. Wie im Zusammenhang mit den Instruktionen beschrieben, sind die Begriffe, die in der Darstellung der unterschiedlichen Bildschirmoberflächen genutzten werden, ein deutlicher Ausdruck des sprachlichen Codes, der Ökonomie- und Spielmodul miteinander verbindet.

Die Beispiele für das situative Zusammenspiel von unterschiedlichen Ethnomethoden der Handelnden zeigten auf, wie Kommunikations- und Bewegungsrechte der ExperimentatorInnen gezielt genutzt werden, um die Handlungskoordination im Labor zu unterstützen. Die Verknüpfung von Spiel-, Ökonomieund Experimentalmodul wird hier interaktiv erzeugt, bestärkt und verankert. Das gekonnte Zusammenspiel von technischen, körperlichen und verbalen Aspekten basiert hier maßgeblich auf den praktischen Wissens- und Erfahrungsbeständen der ExperimentatorInnen. Es zeigt sich, wie die unterschiedlichen kommunikativen Modalitäten situativ durch die Mitglieder des Laborteams und ihre kommunikativen Handlungen miteinander verbunden werden. Sie befördern damit nicht nur die geplante sequenzielle Durchführung der Handlungsabfolge und damit das Doing der speziellen Laborsituation. Die Kombination aus technisch vermittelten Eingaberestriktionen und performativen Praktiken und Kontrolltechniken der Labormitglieder trägt maßgeblich zum Doing von rationalen und regelkonformen Verhaltenserwartungen gegenüber den ProbandInnen bei. Die Reduktion der auf den Benutzeroberflächen präsentierten Inhalte und die Objektivation der Handlungen der ProbandInnen durch die von ihnen getätigten Eingaben bedingen dabei auch, dass der Fokus verstärkt auf monetäre Aspekte des Entscheidungsspiels gelegt werden kann. Die Kombinationen der unterschiedlichen kommunikativen Handlungen im Labor führt den ProbandInnen zudem immer wieder die Entscheidungsabhängigkeit ihrer Gewinnmöglichkeiten vor Augen. Diese Aspekte tragen somit zugleich stark dazu bei, dass im ökonomischen Laboratop eine soziale Situation geschaffen wird, die ein Doing rationaler und ökonomischer Verhaltenserwartungen an die ProbandInnen befördert.

\section{Der Umgang mit Regelüberschreitung und situativangemessene Sanktionen}

Die nachfolgenden Beispiele problematischer oder krisenhafter Situationen der Durchführung von Experimenten zeigen auf, wie die Orchestrierung von verschiedenen Ethnomethoden der ExperimentatorInnen situativ eingesetzt wird, um zur Durchsetzung und damit zum Doing regelkonformer Verhaltenserwartungen 
gegenüber den ProbandInnen beizutragen. Sie induzieren und verankern maßgebliche Aspekte des Spielmoduls. Im interaktiven Vollzug der Experimente sorgen sie für die Durchsetzung und Einhaltung der formellen und informellen Regeln des Spiels. Zugleich wird an diesen Beispielen besonders deutlich, dass die Produktion und Reproduktion der situativen Ordnung im Labor ein Ergebnis der gemeinsamen Handlungen und situativen Aushandlung der Beteiligten ist und die ProbandInnen, ebenso wie die ExperimentatorInnen, Erwartungen an Abläufe und Handlungsspielräume im Labor haben.

\subsubsection{Beispiel 1:Zu spätes Erscheinen}

Anhand eines ersten empirischen Beispiels wird dargestellt, dass auch die ProbandInnen die Regeln in ihren Handlungen antizipieren und sich der möglichen Sanktionen bei Zuwiderhandlung bewusst sind. Zugleich zeigt sich, wie gewissenhaft die Regelbefolgung der Teilnehmenden bereits bei der Anmeldung durchgesetzt wird.

In der beobachteten Situation hatten die TeilnehmerInnen gerade das Labor betreten und die Tür hinter ihnen wurde geschlossen, um mit dem geplanten Experiment zur angegebenen Zeit zu beginnen. Ein junger Mann kam schnellen Schrittes auf den Mitarbeiter des Laborteams zu, der sich noch vor der Tür des Experimentallabors befand. Ohne weitere Begrüßung begann der junge Mann sofort, sich für seine Verspätung zu entschuldigen. Er beschrieb detailreich die Gründe für seine Verspätung und bat inständig darum, in seiner Datei keinen Negativeintrag zu vermerken. Die Diskussion mit dem Mitarbeiter des Laborteams dauerte mehrere Minuten an. Wegen der Kürze seiner Verspätung einigte man sich darauf, dass er zwar nicht mehr die 5 Euro Show-up fee erhalten sollte, doch der Malus in der Liste zurückgenommen werde. Der junge Mann war darüber sehr dankbar und beteuerte, dass er beim nächsten Mal mehr als pünktlich sein werde.

Aus der Beschreibung der beobachteten Situation wird deutlich, dass der junge Mann bereits begann, sich zu rechtfertigen und zu entschuldigen, bevor der Mitarbeiter des Labors ihn mit seiner Regelverletzung konfrontierte. Dies kann als Indiz dafür gedeutet werden, dass er den hohen Stellenwert, der der Regelbefolgung im Rahmen der Experimente beigemessen wird, als Hintergrundwissen in dieser Situation aktualisierte und die Konsequenzen seiner Handlung bereits antizipierte. Auch in der Reaktion des Mitarbeiters zeigte sich eine reflexive Bezugnahme auf das standardisierte Vorgehen bei den Experimenten. Es bedurfte keiner Erklärung, dass das Zuspätkommen des Teilnehmers mit einer Sanktion einhergehen würde. Auch wurde als Hintergrundwissen beider vorausgesetzt, dass stets mehr 
ProbandInnen als benötigt zu den Experimenten eingeladen werden und alle ProbandInnen, die rechtzeitig erscheinen, jedoch nicht am Experiment teilnehmen können, Anspruch auf die Show-up fee von 5 Euro als Aufwandsentschädigung haben.

Auch wenn die geschilderte Episode nicht Teil einer Interaktion im Labor ist, so beschreibt sie doch anschaulich, wie die InteraktionsteilnehmerInnen aktiv an der Produktion der situativen Ordnung teilhaben, die für das Laborexperiment charakteristisch ist. Es handelt sich um ein Doing regelkonformer und rationaler Verhaltenserwartungen, welche an die ProbandInnen gestellt werden. Die Aushandlung der beiden Interaktionspartner befasst sich nicht mit der Frage, ob sanktioniert werden sollte, sondern nur damit, welche Form und welches Ausmaß aufgrund der kurzen Verspätung angemessen wären. Die gewählte Sanktion besteht in der Nichtzahlung der üblichen Show-up fee und nimmt somit Bezug auf den monetären Anreizfaktor und die typische Verknüpfung von Spiel- und Ökonomiemodul.

\subsubsection{Beispiel 2: Mindestlohn}

Anhand des zweiten Beispiels wird aufgezeigt, dass auch ProbandInnen Erwartungen an das Geschehen im Labor und die ihnen zugestanden Handlungsspielräume haben. Wie das nachfolgende Beispiel aufzeigt, bildet die Entscheidungsabhängigkeit der Gewinne, die in einem Experiment erzielt werden können, eine dieser Erwartungen. Zugleich wird deutlich, dass auch die technisch vermittelte sequenzielle Strukturiertheit des Handlungsablaufs zur Herausforderung für die geplante Durchführung eines Experiments werden kann.

In einem Experiment zum Thema Mindestlohn wurden jeweils 5er Gruppen von ProbandInnen gebildet. In jeder Gruppe gab es drei Rollen (Konsument, Firma und Arbeitnehmer), von denen die ProbandInnen je eine zufällig zugewiesen bekamen. Jede Spielergruppe bestand aus einem Konsumenten, zwei Firmen und jeweils einem zugeordneten Arbeitnehmer pro Firma. ${ }^{78}$ In einem der beobachteten Experimente dieser Versuchsanordnung gingen ProbandInnen, die die Rolle der Firmen einnahmen, nach einiger Zeit dazu über, gar keinen Lohn an ihre Arbeitnehmer zu zahlen. Diejenigen, die die Rolle der Arbeitnehmer spielten, hatten durch den im Programm vorgegebenen Rahmen keine Einflussmöglichkeit, etwas dagegen zu unternehmen. Ihr Anteil am Gang des Experiments bestand nur darin, in jeder Runde erneut auf den OK-Button zu drücken und die angezeigten Ergebnisse der Wertungsrunde zu bestätigen.

\footnotetext{
${ }^{78}$ Eine detailliertere Beschreibung der Versuchsanordnung des Experiments findet sich weiter oben im Abschnitt zur technischen Realisierung des sequenziellen Ablaufs (Abschnitt 5.4.5).
} 
Nach einigen Runden stockte der Fortgang des Experiments auf einmal. Alle TeilnehmerInnen warteten. Es folgte die allgemeine Aufforderung eines Experimentators, der alle ProbandInnen bat, darauf zu achten, auf ihrem Bildschirm den OK-Button zu drücken. Die Anwesenden warteten jedoch weiterhin. Mit Hilfe des Übersichtsbildschirms (Clients' Table) konnte ermittelt werden, an welchem Computer der Proband saß, der das Experiment zum Erliegen brachte. Einer der Labormitarbeiter trat an seinen Arbeitsplatz heran und fragte nach dem Grund für die Verzögerung. Proband Martin, dem die Rolle eines Arbeitnehmers zugewiesen worden war, hatte aus Unmut über die ihm zugewiesene passive Rolle einfach unterlassen, den besagten OK-Button zu drücken und ließ alle anderen ProbandInnen deshalb warten. Er wurde von dem Experimentator aufgefordert, den OK-Button nun zu drücken, um den Fortgang des Experiments zu ermöglichen. Er tat, wie ihm geheißen. Doch in der nächsten Runde gab es wieder eine Verzögerung durch den Probanden Martin, der erneut alle anderen zum Warten nötigte. Wieder erschien ein Mitglied des Laborteams an seinem Arbeitsplatz und betonte nachdrücklich, dass er den Fortgang des Experiments bitte nicht weiter stören solle. Für den Rest des Experiments blieb ein der Experimentator hinter dem Platz von Martin stehen und beobachte direkt, ob er die geforderte Bestätigung der Rundenergebnisse zeitnah vollzog. Die Anonymität des Probanden, seiner Rolle und seiner Entscheidungen wurde damit aufgrund der krisenhaften Situation aufgehoben.

Durch die krisenhaften Situationen wird ersichtlich, dass auch die ProbandInnen bestimmte Erwartungen an ihren Handlungsspielraum in den Laborexperimenten haben und in ihrem Handeln darauf Bezug nehmen. Die Erwartung des renitenten Probanden Martin an die normale Situation des Experiments bestand darin, dass er selbst maßgeblich beeinflussen könne, wie viel er im Experiment gewinnen könne. Dies war in der ihm zugewiesenen Rolle jedoch nicht möglich. Durch sein Handeln und seine Äußerungen weist er aus, dass die entscheidungsabhängige Entlohnung, als charakteristische Verknüpfung des Spielund das Ökonomiemoduls, ein antizipiertes Rahmungselement des ökonomischen Laboratops bildet. Diese Erwartung an den geltenden Rahmen wird jedoch in der ihm zugewiesenen Rolle nicht erfüllt. ${ }^{79}$ Die Entscheidungsabhängigkeit der

\footnotetext{
${ }^{79}$ Ein grundlegender Anspruch der Forschenden besteht darin, die Spielsituation für die ProbandInnen in ihren zentralen Aspekten abzubilden. Im Fall des Mindestlohns nahmen die Forschenden an, dass die Anwesenheit einer realen Person als ArbeitnehmerIn das Verhalten der ProbandInnen in der Rolle der Firmen und Konsumenten beeinflussen könnte. Deshalb war die Rolle des Arbeitnehmers Teil der Entscheidungssituation im Labor, obwohl diese nur als ,Statisten“ agierten. In späteren Treatmentvariationen dieser Experimentserie wurde die Rolle der Arbeitnehmer zur Darstellung der Spielsituation nicht mehr eingesetzt.
} 
Entlohnung liegt in diesem Experiment vor. Die ProbandInnen in der Rolle der Firmen und Konsumenten fällten hier jedoch die Entscheidungen. Die ProbandInnen in der Rolle der Arbeitnehmer waren lediglich mit den Konsequenzen dieser Entscheidungen konfrontiert, welche ihre eigenen Spielgewinne bestimmten. Die Krise der geltenden Rahmenerwartungen des Probanden wurde so durch seine Handlungen zu einer Krise für alle Beteiligten.

Auch das Handeln des Probanden Martin hinsichtlich des ihm verbliebenen Handlungsspielraums im Labor zeigt seine Erwartung an die geltenden Rahmungselemente des ökonomischen Laboratops. Der Proband wählte das Unterlassen einer geforderten Handlung als Ausdruck seines Protests. Auch wenn die Interventionen des Teilnehmers zu einer faktischen Störung und deutlichen Verlängerung des Experiments beigetragen haben, waren seine Handlungen kein Verstoß gegen die strikt einzuhaltenden offiziellen Laborregeln. In den Instruktionen und mündlichen Ansagen der ExperimentatorInnen wurde nicht mitgeteilt, dass es eine zeitliche Begrenzung für die Bestätigung der Rundenergebnisse gibt. Im Sinne der offiziellen Regeln hatte er nicht zuwidergehandelt. Der Proband Martin verstieß jedoch wiederholt gegen die informelle Regel, keine unnötigen Verzögerungen zu verursachen. Dass seine Handlungen ein unerwünschtes Verhalten im Labor darstellten, wurde ihm durch die Hinweise der LabormitarbeiterInnen und die damit verbundenen unterschiedlich starken Methoden der Sanktionierung deutlich gemacht. Da dem Probanden kein offizieller Regelverstoß vorgeworfen werden konnte und er der direkten Aufforderung nachkam, den OK-Button zu drücken, war seine Auszahlung jedoch nicht gefährdet. Der Zusammenhang zwischen einem regelkonformen Verhalten und der Auszahlung, an welchem sich die ProbandInnen orientieren, wird hier deutlich und zeigt, wie Spiel- und Ökonomiemodul sich in der Situation des ökonomischen Laboratops gegenseitig verstärken.

Das Beispiel dieser krisenhaften Situation zeigt, dass zentrale Aspekte wie die Anonymität der Rolle und der Entscheidungen der ProbandInnen gegebenenfalls außer Kraft gesetzt werden, wenn der Fortgang eines Experiments gefährdet ist. Das allgemeine Anliegen eines Experiments ist es, eine Situation zu schaffen, in der die einzelnen ProbandInnen anonym und individuell ihre Entscheidungen treffen. Sie sollen vor äußeren Störfaktoren und der Wirkung unerwünschter Versuchsleiter-Effekte bewahrt werden. Massive öffentliche Eingriffe durch die LabormitarbeiterInnen laufen diesen Ansprüchen deutlich zuwider. Die Reaktion der Labormitglieder zeigt dem Probanden deutlich, dass er im Rahmen des Experiments nicht vollkommen anonym agiert, da es für das Laborteam ein Leichtes war, ihn als ,Delinquenten“ auszumachen. Die anschließende körperliche Präsenz des Labormitarbeiters stellt eine drastische Sanktionsmaßnahme dar, die 
sich aus diesem devianten Verhalten ergibt. Es wird so überwacht, dass auch im Fortgang des Experiments diese unerwünschten Handlungen unterbunden werden können. Die massive Beeinflussung dieses Probanden erscheint als situativ angemessene Lösung, um den Fortgang des restlichen Experiments zu gewährleisten und zu ermöglichen, dass zumindest die anderen Probanden zur „Normalität“ des Experiments zurückkehren können. Auch für die anderen ProbandInnen ist diese Sanktionsmaßnahme deutlich sichtbar. Die Performanz des Handelns der Labormitglieder ist Ausdruck der Bewegungsrechte und des Handlungsspielraums, welche die Labormitglieder zur Aufrechterhaltung der Experimentsituation nutzen können. Den ProbandInnen wird durch das Handeln der ExperimentatorInnen zudem aufgezeigt, dass auch informelle Regeln eingehalten werden müssen und ein Zuwiderhandeln nicht geduldet wird. Die praktischen Methoden der LabormitarbeiterInnen können dabei als Aspekte des Doings eines regelkonformen Verhaltens der ProbandInnen verstanden werden. In diesem spezifischen Fall handelt es sich um praktische Methoden zur Durchsetzung der informellen Regel „unnötige Verzögerungen des Experiments unterbinden“.

\subsubsection{Beispiel 3: Chatverbot}

Der zweite Beispielfall thematisierte die Überschreitung einer informellen bzw. nicht eindeutig formulierten Regel. Im Unterschied dazu handelt es sich im dritten Beispiel um eine in den Instruktionen klar kommunizierte Regel, deren unbedingte Geltung vom devianten Probanden falsch eingeschätzt wurde.

Die nachfolgende Beobachtung entstammt einer Session der Treatmentvariation „Vier-gewinnt“ der Experimentserie „Endowment-Effekt-USB-Stick“. In der beobachteten Situation hatten die ProbandInnen alle 16 Runden lang individuell Preise für den Kauf und Verkauf von Lotterielosen festgelegt. Im Anschluss daran wurde das Spiel „Vier-Gewinnt ${ }^{\star 80}$ am Computer gespielt. Jeweils zwei Teilnehmende bildeten, über das Netzwerk koordiniert, ein Spielerpaar. ${ }^{81}$ Die beiden SpielpartnerInnen waren füreinander anonym. In den Instruktionen wurden sie darüber informiert, dass sich ihre SpielpartnerIn ebenfalls im Laborraum befindet. Das Setzen der einzelnen Spielsteine erfolgte immer abwechselnd. Bei einem

\footnotetext{
${ }^{80}$ Es handelt sich hier um ein bekanntes Strategiespiel. Das Spielfeld umfasst 7 Spalten und 6 Reihen, die übereinander angeordnet sind. Spielsteine werden von oben in das Spielfeld eingeworfen und rutschen an die tiefstmögliche Reihe. Dem Namen des Spiels entsprechend, besteht das Ziel darin, eine Reihe von vier Spielsteinen zu bilden. Diese kann diagonal oder horizontal verlaufen.

${ }^{81}$ Zur technischen Umsetzung dieser Phase im Experiment wurde das Programm Mustrum genutzt.

URL: https://www.mustrum.de/mustrum.html, (Zugriff am 16.11.2018).
} 
Unentschieden gewann die SpielerIn die Partie, die nicht den ersten Stein gesetzt hatte. Die Spielpaare sollten nun so lange spielen, bis eine der beiden Parteien vier Siege erzielt hatte. Die GewinnerIn des Spielerpaares sollte als Preis ein Objekt erhalten. Aus technischen Gründen war es nicht möglich, die integrierte Chatfunktion des Vier-Gewinnt-Spiels zu deaktivieren. Um die Kommunikation zwischen den SpielpartnerInnen als mögliche Störvariable auszuschließen, wurde in den Instruktionen explizit und nachdrücklich darauf verwiesen, dass es den SpielerInnen untersagt sei, diese Chatfunktion zu nutzen. Für den Fall eines Verstoßes gegen diese Regel wurde damit gedroht, dass die GegenspielerIn zur GewinnerIn des Spielerpaares erklärt werden würde und die SpielerIn, die den Regelverstoß begangen habe, das Labor ohne Bezahlung zu verlassen habe. In einem Experiment ereignete sich nun folgender Zwischenfall:

Ein Proband Sven meldete sich und berichtete, dass sein Spielpartner nicht mehr reagiere. Mittels des Übersichtsbildschirms der ExperimentatorInnen konnte schnell bestimmt werden, welche der TeilnehmerInnen der Spielpartner des betreffenden Probanden war. Der Spielpartner Marc erklärt dem Mitglied des Laborteams sein Handeln damit, dass sein Mitspieler (Sven) im Chat etwas geschrieben habe und er aufgrund dessen nicht mehr reagiert habe. Der Inhalt der Kommunikation bezog sich jedoch nicht auf das Objekt, das als Preis im VierGewinnt-Spiel gewonnen werden konnte. Sven teilt Marc mittels des Chats mit, dass sie aufgrund des Patts einfach ein neues Spiel beginnen könnten. Anliegen des Probanden Sven war es, das Spiel seines Spielpaares zu beschleunigen. Dies änderte aber nichts daran, dass der Proband Sven, der die Chatfunktion genutzt hatte, von einem Experimentator aufgefordert wurde, das Labor ohne Bezahlung zu verlassen. Marc, der andere Proband, wurde hingegen zum Gewinner seiner Gruppe erklärt. Die drei an der Handlungssequenz beteiligten Akteure zeigten hier jeweils ein anderes Verständnis der situativen Regeln, an denen sich ihr Handeln orientierte.

Diese Sequenz zeigt, wie regelkonformes Verhalten im Sinne eines Doings von rationalen und regelkonformen Verhaltenserwartungen durch die Ethnomethoden der Labormitglieder und durch die ProbandInnen selbst gemeinsam situativ produziert wird. Der Proband Sven wird auf seine Regelverletzung erst durch die Handlungen der anderen Akteure aufmerksam gemacht. Sein Abweichen von den Regeln wird erst durch die darauf bezogenen Handlungen der anderen Beteiligten als solche konstruiert. Die ausgeführte Sanktion des Labormitglieds wird durch die Bezugnahme auf die verbal und schriftlich kommunizierten Regeln legitimiert. Auf diese wird als geteiltes Hintergrundwissen Bezug genommen. Der zweite Proband Marc kann durch die Handlungen des Experimentators erkennen, dass er „,richtig“ gehandelt hat, indem er die Handlungsaufforderung von Sven, ein neues 
Spiel zu beginnen, ignorierte. Dass Marc diesen Verstoß den ExperimentatorInnen gegenüber nicht anzeigte und sein Gegenüber warten ließ, ist in dieser Situation für den agierenden Experimentator irrelevant.

In der beobachteten Situation wird deutlich, dass hier verschiedene Beurteilungen des geltenden Rahmens vorlagen. Es zeigt sich hier die Differenz zwischen dem prozessualen Rahmen (frame-in-use) des Probanden Sven und dem Referenzrahmen des Laboratops. Beide enthalten bestimmte Rahmenelemente und weisen diesen in der jeweiligen Situation des aktiven Sense-making bestimmte Rahmungsrelevanzen zu. Das Verständnis der Situation des Probanden Sven bestand in einem Versuch der Verschiebung des offiziellen Rahmens und damit der offiziell geltenden Regeln. Die Geltung der allgemeinen Regel, den Chat nicht zu benutzen, sollte zugunsten der informellen Regel der Zeitersparnis abgewandelt werden. Während der Proband Sven im Sinne Goffmans eine (situative) Modulation des Referenzrahmens vollzog, wurde diese von seinem Mitspieler Marc nicht vollzogen und auch nicht anerkannt. Die Deutung des Experimentators und auch des zweiten Probanden Marc widersprachen der Verschiebung des Rahmens durch Sven. Eine Umdeutung durch den Probanden Sven war hier in diesem Sinne nicht zulässig. Aus diesem Grund entstand eine krisenhafte Situation. Da das Kommunikationsverbot aus Sicht des Experimentators einen zentralen Stellenwert für die Reliabilität des Experiments und seiner Ergebnisse bildet, wurde der Verstoß des Probanden Sven von ihm scharf sanktioniert. Die Deutungsdifferenz zwischen Sven und dem Experimentator, die in anderen Fällen einen Faux Pax hätte darstellen können, war hier die Grundlage für eine Szene. Für den Probanden Sven bot sich keine Chance, die Situation zu reparieren und seine Handlungen zu korrigieren.

Regelkonformes Handeln im Sinne des Sprach- und Verhaltensspiels der Forschenden wird verlangt, auch wenn die situative Bewertung der ProbandInnen - die Relevanz also, die sie der Regel beimessen - zu individuell unterschiedlichen Bewertungen führt. Denn das Anliegen von Sven war es ja eigentlich, der informellen Regel „unnötige Verzögerungen vermeiden“ zu entsprechen. Er verstieß dabei jedoch gegen eine offizielle Regel des "Chatverbots“, welcher ein deutlich höherer Stellenwert beigemessen wurde. Sven hatte seinen Handlungsspielraum in der Auslegung der offiziell kommunizierten Regel falsch eingeschätzt. Die Reaktion der anderen Beteiligten zeigte nun, dass die Situationsdefinition von Sven unangemessen war und folgenreiche Konsequenzen nach sich zog. Um von Seiten des Experimentators die Relevanz und die Aufrechterhaltung der Regeln eindeutig zu kommunizieren, nutzte dieser eine Sanktionsmöglichkeit, die seines Erachtens der Schwere der Regelverletzung angemessen war und verwies den Probanden des Labors. 
Dieses Beispiel zeigt auf, das die Spielräume für informelles Verhalten zwischen den ProbandInnen sehr begrenzt sind. Im Sinne von unkontrollierten Störfaktoren zielen viele Ethnomethoden im Labor gerade darauf ab, diese zu unterbinden oder zumindest zu minimieren. Im Labor sind Interaktions- und Kommunikationsbeziehungen zwischen den ProbandInnen nur im Rahmen der von den ExperimentatorInnen strukturieren Handlungsformen zulässig. Eigenmächtige Erweiterungen des Interaktionsspielraums durch die ProbandInnen werden, wie das Beispiel zeigte, für alle Anwesenden sichtbar und deutlich sanktioniert. Als performativer Sanktionsmechanismus wird dabei, neben der öffentlichen Stigmatisierung des Probanden und seines devianten Verhaltens, zugleich der Verlust des erspielten Gewinns genutzt. Es zeigt sich auch hier, wie das Doing von regelkonformen Verhaltenserwartungen und seine Durchsetzung mit dem Doing von rational-ökonomischen Verhaltenserwartung einhergeht. Der Verlust der erspielten Gewinne wird hier als starkes Sanktionsmedium eingesetzt und verknüpft so Spiel- und Ökonomiemodul auf eine performative Weise: Wer sich nicht an die Regeln des Spiels hält, kann auch nichts gewinnen. Regelkonformes Handeln gewinnt gerade im Licht dieser drohenden Sanktion an Attraktivität.

\subsubsection{Beispiel 4: Gleichzeitige Eingabe}

Anhand eines letzten Beispiels soll abschließend aufgezeigt werden, wie im Verlauf einer Serie von Experimenten Lösungen für krisenhafte Situationen gesucht und umgesetzt werden. Auch hier zeigt sich erneut, wie in der Situation des Experiments eine Aushandlung der situativen Regeln erfolgt. Dieses letzte Beispiel beschreibt eine Beobachtung aus der Treatmentvariation „Vier-Gewinnt“ aus der Experimentserie „Endowment-Effekt-USB-Stick“. Die grundlegende Beobachtungssituation entspricht der in Beispiel 3 eingeführten. Der geplante Handlungsablauf dieser Treatmentvariation bestand darin, dass die ProbandInnen nach der Begrüßung und dem Lesen der Instruktionen in 16 aufeinanderfolgenden Runden den Kauf- oder Verkaufspreis für Lotterielose festlegen sollten. Im Anschluss daran sollte das Spiel „Vier-Gewinnt“ am Computer gespielt werden. Jeweils zwei Teilnehmende bildeten hier, über das Netzwerk koordiniert, ein Spielpaar. Beide Spielpartner waren füreinander anonym. In den Instruktionen wurde ihnen jedoch mitgeteilt, dass sich ihr Spielpartner auch im Laborraum befindet. Wie in Beispiel 3 dargestellt, konnten die ExperimentatorInnen mittels des Übersichtsbildschirms bestimmen, welche beiden Spielpartner zusammengehörten. Die Spielpaare sollten nun so lange spielen, bis jemand von beiden vier Siege erzielt 
hatte. ${ }^{82}$ Das Spielergebnis musste anschließend von beiden SpielpartnerInnen am Computer eingetragen und durch Klicken auf den „OK-Button“ bestätigt werden. Waren die Eingaben der beiden SpielerInnen verschieden, so wurden sie vom Computer erneut gebeten, das richtige Ergebnis einzutragen. Aufgrund der Programmierung des Experiments war es notwendig, dass die Eingabe der zweiten Person innerhalb eines festgelegten Zeitfensters von 30 Sekunden erfolgen musste. Eine zweimalige falsche Eingabe oder einer Überschreitung des Zeitfensters führte zu einem technischen Fehler und einem Absturz des Programms. Zusätzlich wurde in den Instruktionen darauf verwiesen, dass die beiden SpielpartnerInnen den ExperimentatorInnen per Handzeichen anzeigen sollten, wenn einer der Beteiligten vier Mal gewonnen hatte. Erst nach der Überprüfung des Ergebnisses durch das Laborteam sollte die Eintragung am Computer erfolgen.

Es wurden zwei aufeinanderfolgende Sessions durchgeführt, in denen diese Variante des Experiments gespielt wurde. Bereits in der ersten Session trat jedoch der Krisenfall ein. Eine Person des Spielpaares gab sein Ergebnis ein, doch der zweite Mitspieler tätigte und bestätigte seine Eingabe nicht im vorgesehenen Zeitrahmen. Das Programm stürzte als Konsequenz ab. Durch die technische Sachkenntnis meines Kollegen und die Unterstützung der technischen Angestellten des Labors konnten die Daten notdürftig gesichert werden. Das sonst streng befolgte Kommunikationsverbot im Labor wurde in diesem Fall kurzerhand außer Kraft gesetzt, da die technische Mitarbeiterin des Labors öffentlich fragte, bei welchen ProbandInnen das Programm nicht mehr funktionierte. Die sonst klaren Regeln im Labor wurden situativ neu ausgehandelt, sodass nun auch verbale Äußerungen der Teilnehmenden akzeptiert wurden. Durch den Verweis auf den Pilotstatus der Session wurde zugleich eine Begründung für die technischen Probleme gegeben und damit die Veränderung gegenüber den sonst geltenden Laborregeln legitimiert.

In der nachfolgenden Session zeigte sich nun deutlich, mittels welcher Methoden die ExperimentatorInnen eine Erhöhung der Kontrolle der situativen Rahmung dieses Spiel-Aspekts des Experiments anstrebten. Durch die kurze Pause zwischen den Sessions war es nicht möglich, das technische Problem durch eine veränderte Programmierung zu beheben. Aus diesem Grund wurde das Problem der ,gleichzeitigen Eingabe ' der ProbandInnen durch Methoden der situativen Handlungskoordinierung und eine stärkere Explizierung der geltenden Spielregeln in den schriftlichen Instruktionen gelöst. Der Text der Instruktionen

\footnotetext{
${ }^{82}$ In den Instruktionen wurden die ProbandInnen darüber informiert, dass die Person, die das Spiel gewonnen hatte, am Ende dieser Spielphase als Preis ein Objekt erhalten würde.
} 
wurde dazu abgewandelt. Es wurde nun nicht nur darum gebeten, die ExperimentatorInnen davon per Handzeichen in Kenntnis zu setzten, wenn einer der Beteiligten viermal gewonnen hatte. Stattdessen wurde dick gedruckt darauf verwiesen, dass eine Eintragung des Spielergebnisses erst getätigt werden durfte, wenn ein Mitglied des Laborteams dies gestattet hatte. Bei Zuwiderhandlung bestünde sonst die Gefahr, dass die betroffenen ProbandInnen von dem weiteren Experiment ausgeschlossen und alle bisher erspielten Gewinne verlieren würden. Vor Beginn des Vier-Gewinnt-Spiels wurde diese Information erneut und nachdrücklich in verbaler Form durch ein Mitglied des Laborteams wiederholt. Mein Kollege und ich blieben zudem bei je einem der Spielpartner am Platz stehen, bis gesichert war, dass die Eingaben beider sich entsprachen und vom Computerprogramm akzeptiert wurden. Durch Kopfnicken signalisierten wir uns dabei gegenseitig, dass eine gleichzeitige Eingabe stattfinden könnte und kontrollierten so die zeitliche Koordination der Handlungen der zusammengehörenden ProbandInnen. Der reibungslose Ablauf dieses Aspekts des Experiments wurde in diesem Fall durch die körperliche Präsenz der ExperimentatorInnen und ihre visuelle Kontrolle der erfolgreichen Eingabe abgesichert. Zudem wurden die ProbandInnen durch den Verweis auf einen potenziellen Verlust des erspielten Gewinns zu einem regelkonformen Handeln animiert.

Der nachdrückliche Hinweis in den Instruktionen, dass eine Eingabe, die nicht von einem Mitglied des Laborteams genehmigt wurde, zum Ausschluss vom Experiment und zum Verlust des Gewinns führen würde, löste jedoch bei den Teilnehmenden Verunsicherung aus. Dies wurde dadurch deutlich, dass sich zu Beginn des Experiments, in der Phase des Lotteriespiels, vermehrt Teilnehmende per Handzeichen meldeten. Sie taten dies jedoch nicht, um eine Frage-AntwortInteraktion einzuleiten, wie bei dieser labortypischen performativen Handlung zu vermuten gewesen wäre. Die ProbandInnen verwiesen vielmehr darauf, dass sie annahmen, dass jede ihrer Eingaben erst durch die Erlaubnis eines Labormitglieds legitimiert werden müsse. Als dies in der Beantwortung der Nachfragen vor Ort richtiggestellt wurde, begründeten die Teilnehmenden ihr Vorgehen damit, dass sie nur sicher gehen wollten nichts falsch zu machen und so versehentlich ihre weitere Teilnahme am Experiment zu gefährden.

In den nachfolgenden Durchführungen dieses Experiments wurde das Problem auf technische Weise behoben. Nach jeder Runde mussten die ProbandInnen auf einer aktiven Benutzeroberfläche angeben, ob sie oder ihr Gegenspieler die Spielrunde gewonnen hatten. Die Ergebnisse der einzelnen Spielrunden wurden durch das Programm selbst aufsummiert. Der Punktestand wurde den ProbandInnen nach jeder Runde angezeigt. Die Spielerpaare spielten so lange, bis einer der 
Spielenden vier Gewinne erzielt hatte. Im Anschluss wurde eine Bildschirmoberfläche angezeigt, die die Teilnehmenden darüber informierte, dass sie Gewinner oder Verlierer des Spiels seien.

In den nachfolgenden Sessions der Treatmentvariation, in denen das technische Problem behoben war, zeigten sich deutliche Differenzen in der Reaktion der ProbandInnen. Hier wurde das Verhalten der Teilnehmenden weder so offensiv durch die körperliche Präsenz der ExperimentatorInnen kontrolliert, noch wurde nachdrücklich und performativ auf die Sanktion bei Zuwiderhandlung und damit auf den Verlust der erspielten Gewinne hingewiesen. Die oben beschriebene anfängliche Verunsicherung der Teilnehmenden zeigte sich hier nicht. Keine der Nachfragen der ProbandInnen thematisierte, ob alle Entscheidungen durch das Laborteam legitimiert werden müssten. Auch technisch traten keine weiteren Störungen mehr auf. Alle beteiligten ProbandInnen bewerkstelligten die koordinierte und übereinstimmende Eingabe ihrer Spielergebnisse.

Hinsichtlich der eingesetzten Methoden zur interaktiven Handlungskoordinierung ist dies ein anschauliches Beispiel dafür, wie unterschiedlich auf diese Probleme reagiert werden kann. Körperliche Präsenz der ExperimentatorInnen und visuelle Kontrolle ermöglichten es, das Problem der Handlungskoordination situativ zu lösen. Zugleich wurde jedoch ersichtlich, welchen Einfluss der Einsatz performativer Methoden durch die ExperimentatorInnen auf die gesamte situative Rahmung des Experiments ausübt. Der deutliche Verweis auf den möglichen Verlust des Gewinns und die Gefahr des Ausschlusses vom weiteren Experiment erscheint maßgeblich für die Verunsicherung der ProbandInnen im oben dargestellten Beispiel.

Wie die Beispiele verdeutlichten, ist die Rahmenbildung im ökonomischen Laboratop ein interaktives Produkt der Handelnden. Auch die ProbandInnen haben spezifische Erwartungen an das Geschehen im Labor. Sie antizipieren Rahmenaspekte und weisen diesen situationsspezifische Relevanzen zu. Diese erweisen sich als prägend für ihre eigene Situationsdefinition und die Handlungsspielräume, die die Teilnehmenden in spezifischen Situationen für ihr eigenes Handeln sehen. In Fällen, in denen die relevanten Rahmenaspekte und ihre situative Hierarchisierung von ProbandInnen und Labormitglieder eine ausreichende Übereinstimmung besitzen, zeigt sich das Handeln beider Gruppen in einer ,harmonischen“ Anschlussfähigkeit. Welche Konsequenzen sich für die ProbandInnen und das Laborgeschehen ergeben können, wenn die Rahmungsrelevanzen zu stark voneinander abweichen, zeigten insbesondere die Beispielfälle des „Chatverbots“ und „Mindestlohns“ auf. Im Beispielfall des „Chatverbots“ führte die Fehldeutung des Probanden Sven hinsichtlich der Auslegung der im 
Labor kommunizierten Regeln zum Verweis aus dem Labor und zum Verlust seiner erspielten Gewinne. Das Beispiel „Mindestlohn“ hingegen zeigte auf, dass die Erwartung hinsichtlich des geltenden Rahmens kein rein situatives Phänomen ist. Vielmehr wurde die problematische Situation dadurch ausgelöst, dass der Proband Rahmungselemente als relevant antizipierte, die sich in der Situation des Experiments nicht einlösen ließen. Im beobachteten Fall war es die Annahme, dass er durch seine eigenen Handlungen Einfluss auf seine Gewinnmöglichkeiten nehmen könnte. Dass diese Erwartung nicht erfüllt wurde, gab den Anstoß für sein krisenhaftes Handeln. Zugleich zeigte sich in der Ausführung seines Protestes eine gekonnte Bezugnahme auf die konstitutiven Rahmenelemente der Laborsituation. Sein ,Nicht-Handeln“ erfüllte seinen performativen Zweck und provozierte eine Irritation des Laborgeschehens. Zugleich konnte ihm jedoch kein , offizieller' Verstoß gegen die kommunizierten Verhaltensregeln vorgeworfen werden, sodass er seine Auszahlung nicht gefährdete. Aus Sicht der Labormitglieder zeigte sich hier bei beiden Probanden eine Überschreitung des zulässigen Handlungsspielraums, welcher den Probanden in ihrer Rolle zugemessen wird. Ihren eigenen Handlungs-, Bewegungs- und Kommunikationsrechte entsprechend, reagierten sie auf diese Überschreitung mit Sanktionsmaßnahmen, die sie für situationsangemessen erachteten.

Zudem wurde ersichtlich, dass die ExperimentatorInnen über vielfältige kommunikative Methoden und Eingriffsmöglichkeiten verfügen, um die Durchführung der Experimente zu gewährleisten und situativ auf aufkommende Probleme zu reagieren. Die ExperimentatorInnen bemühten sich, Störungen zu überwinden und abweichendes Verhalten der ProbandInnen ,in Ordnung zu bringen“ und sie $\mathrm{zu}$ rationalem und regelgeleitetem Verhalten $\mathrm{zu}$ motivieren. Eine Gemeinsamkeit der dargestellten Beispiele besteht darin, dass die performativen Handlungen der ExperimentatorInnen maßgeblich auf das Doing rationaler und regelkonformer Verhaltenserwartungen der ProbandInnen zielen. Das Einhalten, Bestärken und Verankern der Regeln des geltenden Spiels im Labor zeigt sich hier als ein dominantes Handlungsmotiv. Die performativen Eingriffe von Seiten der ExperimentatorInnen sind dabei unterschiedlich stark und reichen von freundlichen Hinweisen bis zum Verweis der ProbandInnen aus dem Labor. Das typische Sanktionsmittel, welches eingesetzt wird, um Regelüberschreitungen zu vermeiden oder $\mathrm{zu}$ ahnden, ist der Verlust von Gewinnmöglichkeiten oder bereits erspielten Gewinnen. Grundlegend ist es jedoch nicht im Interesse der Labormitglieder, solche starken Sanktionsmaßnahmen zu vollziehen wie im Beispielfall des „Mindestlohn-Experiments“ und des „Chatverbots“. Diese gefährden die Geltung des Experimentmoduls. Gerade unter Beachtung des Anspruchs des Konstanthaltens der Randbedingungen zielen die ExperimentatorInnen vielmehr darauf ab, 
deutliche und öffentlich sanktionierende Eingriffe zu vermeiden, um nicht Gefahr zu laufen, undokumentierte Störvariablen zu produzieren, die die Ergebnisse der Datenerhebung beeinflussen könnten. Vielmehr werden standardisierte und am besten technisch gestützte Lösungen herangezogen, um potenzielle Störfälle zu vermeiden und die Handlungskoordination im Labor zu erleichtern.

\subsubsection{Zusammenfassung der Analyseebene der situativen Realisierung}

Im Analyseabschnitt der situativen Realisierung wurden solche typischen kommunikativen Handlungsmuster herausgestellt, die zur situativen Handlungskoordination beitragen. Es wurden unterschiedliche kommunikative Handlungen und Objektivationen identifiziert, die in ihrem Doing die kommunikative Konstruktion des ökonomischen Laboratops auf verschiedene Weise unterstützen und auf diese Weise die Induzierung und Verankerung zentraler Rahmenelemente unterstützen. Materielle und technische Aspekte der Handlungssituation erweisen sich dabei als ebenso zentral wie die kommunikativen Handlungen der anwesenden Personen. Nachfolgend wird kurz zusammengefasst zu welchen Rahmenmoduln die einzelnen Merkmale der situativen Realisierungsebene maßgeblich beitragen.

Anhand der nummerierten Spielchips wurde dargestellt, wie durch den gezielten Einsatz materieller Objekte Probleme der individuellen Handlungskoordination mit standardisierten Mitteln und zugleich auf situative Weise bewältigt werden. Die nummerierten Spielchips stellen objektivierte Handlungsanweisungen für die ProbandInnen dar und sind Teil des Doings von rationalen und regelkonformen Verhaltenserwartungen. Sie geben vor, welchen Arbeitsplatz einzelne ProbandInnen im Labor aufsuchen sollen. Der Einsatz der Spielchips ist ein Aspekt des Doings der spezifischen Laborsituation. Es wurde hier deutlich, welche Bedeutung der Vereinzelung der ProbandInnen bei der Umsetzung der außenstrukturellen Ansprüche wie der Randomisierung und Anonymisierung im Vollzug des Experiments zukommt. Die nummerierten Spielchips tragen damit sowohl zur Verankerung des Spiel(er)-Moduls als auch zum Experiment-Modul bei.

Die Aufgabe der Mitglieder des Laborteams besteht darin, die geplante Durchführung der Experimente im Labor zu überwachen und performativ durch ihre kommunikativen Handlungen zu stimulieren und zu unterstützen.

In diesem Zusammenhang wurde auf die unterschiedlichen Kommunikationsund Bewegungsrechte von Labormitgliedern und ProbandInnen hingewiesen. Diese rollenspezifischen Unterschiede werden nicht nur durch die schriftlichen und mündlichen Instruktionen vermittelt, sondern in den Interaktionen zwischen 
ProbandInnen und ExperimentatorInnen im Labor stetig situativ produziert und reproduziert und tragen gemeinsam zum Doing rationaler und regelkonformer Verhaltenserwartungen bei. Die Betrachtung der Handlungsprozesse des Stellens und Beantwortens von Fragen oder des Initiierens von neuen Handlungsphasen durch die ExperimentatorInnen stellen typische Muster kommunikativer Handlungen dar, an denen dies ersichtlich wird. Die Ethnomethoden der ProbandInnen und ExperimentatorInnen müssen sich zudem den speziellen räumlich-materiellen Gegebenheiten des Labors anpassen. Dies wurde am Beispiel der Meldung einer Probandin illustriert, welche bemüht war, die visuelle Abschirmung in ihrer Kabine zu überwinden. Die Methode des ,gezielten Rundgangs' ist ebenfalls stark durch die räumlich-materille Struktur des Laborraums geprägt. Zugleich wurde hier verdeutlicht, wie die körperliche Präsenz der ExperimentatorInnen im Labor auf performative Weise zur Handlungskoordinierung und -kontrolle eingesetzt wird. An vielen Beispielen wurde illustriert, wie die Labormitglieder die ihnen zur Verfügung stehenden Handlungsspielräume gezielt im performativen Vollzug ihrer kommunikativen Handlungen nutzen, um das Spiel(er)-Modul durchzusetzen und zu verankern. Auch die ProbandInnen tragen im Sinne des Accountings durch regelkonforme Initiierung von Interaktionen zur Bestärkung und Aufrechterhaltung der Spielregeln im Labor bei.

Auch der Einsatz der technischen Medien zeigt sich als einflussreicher Aspekt hinsichtlich der situativen Realisierung des Handlungsgeschehens im Labor. Die technische Realisierung unterstützt und strukturiert den sequenziellen Ablauf des Laborgeschehens. Die eingesetzten technischen Medien sind Teil des Doings der spezifischen Laborsituation. Durch die Standardisierung der Handlungsabfolgen unterstützen sie die Verankerung des Experimentmoduls. Die Prägkräfte der technischen Medien zeigen ihre Wirkung in der begrenzenden Funktion, die sie auf die Entäußerung, Darstellung und Vermittlung kommunikativer Inhalte haben. Sie bilden aus diesem Grund Aspekte des Doings von rationalen und regelkonformen Verhaltenserwartungen. Durch diese prägende Kraft auf die Strukturierung der Handlungsspielräume trägt die technische Realisierung maßgeblich zur Verankerung des Spiel(er)-Moduls bei. Zugleich wird auf diese Weise die Fokussierung auf die Inhalte unterstützt, die aus ökonomischer Sicht für die Handlungssituation relevant sind. Die technische Realisierung ist Teil des Doings der ökonomisch-rationalen Verhaltenserwartungen und verankert so zugleich das Mikroökonomie-Modul als Rahmungselement der Laborsituation.

Auch auf Seiten der ExperimentatorInnen erweist sich die technische Vermittlung von kommunikativen Inhalten als folgenreich für die situative Koordination ihrer Handlungen. Der kompetente Umgang mit dem Übersichtsbildschirm der ExperimentatorInnen ist eine wichtige Ethnomethode der Labormitglieder. Diese 
trägt entscheidend zum Doing der rationalen und regelkonformen Verhaltenserwartungen bei und unterstützt die Aufrechterhaltung des Spiel(er)-Moduls. Der Übersichtsbildschirm fungiert als ein zentrales Tool der Kontrolle der Situation, wobei die Kontrollfunktion für die ProbandInnen unsichtbar bleibt. Der Übersichtsbildschirm ist zudem ein zentrales Bindeglied zwischen den unterschiedlichen Ethnomethoden der Labormitglieder, die zur Koordinierung und Kontrolle der Handlungssituation im Labor genutzt werden.

Das Zusammenspiel verschiedener Ethnomethoden der Labormitglieder wurde als Orchestrierung bezeichnet. Es wurde hier an verschiedenen typischen Beispielen illustriert, wie durch den Rückgriff auf typische Kombinationen von verbalen, körperlichen und technisch vermittelten kommunikativen Handlungen die situative Handlungskoordination und die Hervorbringung der situativen sozialen Ordnung im Laboratop erfolgte. Zugleich wurde deutlich, dass trotz aller Intentionen und gezielten Beschränkungen von Handlungsspielräumen die Situation des Laborexperiments ein gemeinsames Produkt von ProbandInnen und ExperimentatorInnen ist. Anhand von Beobachtungen problematischer und krisenhafter Situationen wurde dargestellt, dass das Spiel(er)-Modul zwar als Referenzrahmen der Situationsdeutung eingesetzt wird, es jedoch stets einer situativen Auslegung durch die Labormitglieder und ProbandInnen bedarf. Situationsrahmungen und deutungen, die in Übereinstimmung mit den von den Forschenden angestrebten Sprach- und Verhaltensspielen verlaufen, werden positiv bestärkt. Der Versuch der Verschiebung des Referenzrahmens für die Rahmung wird jedoch negativ sanktioniert. Auf diese Weise wird angestrebt, mit den Regeln des Spiels auch die Regeln der Situationswahrnehmung und -rahmung zu kommunizieren.

\subsection{Das Qualitative Experiment zur experimentellen Validierung eigener Ergebnisse}

Die Gegenstandsadäquanz angewandter Methoden für die Erforschung eines empirischen Gegenstandes bildet ein zentrales Gütekriterium der qualitativen Sozialforschung (Steinke 2000; Flick et al. 1995; Lamnek 1988). Zentrales Anliegen istes, eine passende Methode für das Forschungsdesign, die Datenerhebung und -analyse zu finden, die den Eigenheiten des zu untersuchenden Forschungsgegenstands entspricht. Die Fokussierung der qualitativen Forschung auf natürliche Situationen geht damit einher, dass Eingriffe in die Erhebungssituation diesem Prinzip zuwiderlaufen. Doch die Forderung der qualitativen Sozialforschung, Phänomene in einer natürlichen Situation mittels einer gegenstandsadäquaten Erhebungsmethode zu untersuchen, stößt dort an ihre Grenzen, wo sich der interessierende Forschungsgegenstand einer 
direkten Beobachtung entzieht. Wie in der Entwicklung der konzeptionellen Perspektive dieser Arbeit dargestellt, war mein eigener Forschungsprozess von dem Spannungsverhältnis verschiedener Forschungsstile und -ansprüche geprägt. Das iterativ-zyklische Vorgehen von Datenerhebung und -auswertung und forschungsleitende Ansprüche, wie die des ethnomethodologischen ,,unique adequacy requirements" (Garfinkel \& Wieder 1992), führten zu einer intensiven Beschäftigung und Suchbewegung hinsichtlich der Methodenwahl sowie den Möglichkeiten und Grenzen der Anwendung. Im Zuge des Forschungsprozesses wurde eine Kombination unterschiedlicher Methoden der Datenerhebung eingesetzt, um sich dem Forschungsgegenstand schrittweise explorativ anzunähern.

Wie die bisherige Analyse aufzeigte, konnten die unterschiedlichen Daten dabei gewinnbringend durch ein an der Gattungsanalyse orientiertes Vorgehen trianguliert werden. Im Zuge der Analyse wurden die musterhaften Formen kommunikativen Handelns und die Objektivationen herausgearbeitet, die für die Hervorbringung des ökonomischen Laboratops typisch sind. Zugleich wurde so aufgezeigt, welchen Anteil diese kommunikativen Handlungen und Objektivationen daran haben, einen Rahmen zu induzieren, der es den wirtschaftswissenschaftlichen ForscherInnen ermöglicht, das Verhalten der ProbandInnen als Ausdruck (rationalen) Entscheidens interpretieren und messen zu können.

Anhand der nachfolgenden Anwendung des in Vergessenheit geratenen Verfahrens des qualitativen Experiments soll aufgezeigt werden, welchen Wert eine Infragestellung der üblichen kritisch-ablehnenden Position der qualitativen Forschung gegenüber gezielten Eingriffen haben kann. Das Verfahren des qualitativen Experiments nimmt im Forschungsprozess eine ungewöhnliche Zwitterstellung ein. Es ist zugleich ein Ergebnis der Auseinandersetzung mit dem Feld der experimentellen verhaltensökonomischen Laborforschung und ein Verfahren zur Erkenntnisgewinnung über den Gegenstand selbst. In meiner Auseinandersetzung mit den methodischen Grundlagen des Experiments und der Suche nach einer gegenstandsangemessenen Methode für mein Forschungsvorhaben stieß ich auf die in Vergessenheit geratene Methode des "qualitativen Experiments“ nach Gerhard Kleining (1986), die vielen meiner forschungspraktischen Bedürfnisse zu entsprechen schien. Im Rahmen des Forschungsprozesses wurde die Anwendung dieses Verfahrens erprobt. Zum Zeitpunkt der Konzeption und Erhebung der qualitativen Experimente wollte ich ermitteln, ob dieses Verfahren geeignet wäre, das Framing der Laborsituation aus Sicht der ProbandInnen zu untersuchen. ${ }^{83}$ Die

\footnotetext{
${ }^{83}$ Erste Reflexionen und Erkenntnisse, die durch den Einsatz des qualitativen Experiments im ökonomischen Labor über das Framing der ProbandInnen gewonnen wurden, habe ich bereits in Haus (2018) veröffentlicht.
} 
Erkenntnisse, die durch das qualitative Experiment gesammelt werden konnten, werden im Rahmen der vorliegenden Arbeit argumentativ jedoch etwas anders verwendet. Das qualitative Experiment wird hier nun als ein experimentelles Vorgehen der Validierung eines Aspekts meiner eigenen Forschungsergebnisse herangezogen. Während im Methodenkapitel der Arbeit bereits grundlegende Aspekte des qualitativen Experiments eingeführt wurden, zielt dieser Abschnitt der Analyse darauf ab, die Anwendung des Verfahrens und ihre Ergebnisse zu illustrieren.

Gegenstand der nachfolgend dargestellten experimentellen Variation ist dabei die Informationsdarstellung im Zuge der Instruktionen zu Beginn eines Experiments und die Frage, inwiefern eine Variation dieser Informationen den nachfolgenden Interaktionsprozess des Laborgeschehens beeinflussen würde. Wie im Methodenteil dargestellt, schlägt Kleining als Optionen für einen gegenstandsadäquaten Eingriff sechs konkrete Techniken vor (Kleining 1986: 736 ff.; Kleining 1991: 265). In der hier dargestellten eigenen Serie von qualitativen Experimenten wurde die Technik der Reduktion und Abschwächung ${ }^{84}$ angewendet.

Wie im Methodenteil ausgeführt, fußt die Idee des qualitativen Experiments nach Gerhard Kleining auf der Annahme, dass die Methode der Variation das Grundmotiv des Experiments bildet und nicht die Kausalerklärung von Zusammenhängen zwischen Variablen. ${ }^{85}$ Kleinings Definition folgend, lässt sich das qualitative Experiment dabei wie folgt charakterisieren: „Das qualitative Experiment ist der nach wissenschaftlichen Regeln vorgenommene Eingriff in einen (sozialen) Gegenstand zur Erforschung seiner Struktur. Es ist die explorative, heuristische Form des Experiments“ (Kleining 1986: 724). Der Eingriff in den sozialen Gegenstand ist eine notwendige Bedingung für die aktive Vorgehensweise des Experiments.

Im Folgenden soll geschildert werden, auf welcher Grundlage der gegenstandsadäquate Eingriff ausgewählt und seine Erhebung konzipiert wurde. In der Planung meines eigenen Vorgehens zielte ich darauf ab, einen Eingriff vorzunehmen, der möglichst feldsensitiv und angemessen für die typischen Geschehnisse im Labor war. Im Zusammenhang mit der Nutzung der Videokameras wurde darauf verwiesen, dass die wirtschaftswissenschaftlichen LaborforscherInnen (methodologisch nachvollziehbare) Bedenken hinsichtlich einer bleibenden Beeinflussung

\footnotetext{
${ }^{84}$ Die Techniken der Reduktion und Abschwächung nehmen auf die Frage Bezug, welche Elemente eines Gegenstandes entfernt (Reduktion) oder reduziert (Abschwächung) werden können, ohne dass sich maßgeblich etwas ändert bzw. welche Elemente sensibel auf Eingriffe dieser Art reagieren und sich in der Konsequenz deutlich verändern.

${ }^{85}$ So schreibt schon Ernst Mach (1917/2004), den Kleining als Begründer der Methode des qualitativen Experiments ausweist: „Die Grundmethode des Experiments ist die Methode der Variation“ (Mach 1917/2004: 201).
} 
ihres ProbandInnenpools hegten. Aus diesem Grund waren für mich alle Techniken für einen Eingriff ausgeschlossen, die zu einer starken Störung und Irritation und damit zur Gefährdung der Reliabilität der im Labor erhobenen Daten geführt hätten. In der Auseinandersetzung mit den eigenen Ansprüchen suchte ich nach einem gleichermaßen forschungsethisch vertretbaren und auch inhaltlich relevanten und aufschlussreichen Eingriff. Als Konsequenz dessen wurde der Aspekt der Instruktionen und der Informationsdarstellung für die Variationen ausgewählt. Es handelt sich dabei um einen Aspekt, der für das Laborexperiment sehr charakteristisch ist und dem feldintern für die Durchführung und die Reliabilität des Experiments und seiner Ergebnisse ein hoher Stellenwert beigemessen wird. Für die Anerkennung und Rezeption der Ergebnisse der Erhebung in der Peergroup der Forschenden ist die Einhaltung der Standards bei der Durchführung von Experimenten von maßgeblicher Bedeutung. Werden die Ergebnisse von Experimenten in Publikationen veröffentlicht, so entspricht es dem Standard in der Peergroup der wirtschaftswissenschaftlichen ExperimentalforscherInnen, dass die Instruktionen interessierten LeserInnen ebenfalls freizugänglich gemacht werden. Gleiches gilt für relevante Informationen, die den ProbandInnen zusätzlich gegeben wurden. Bei der Planung von Baseline Treatments (Kontrolltreatments) experimenteller Erhebungen orientieren sich die Forschenden häufig an den Instruktionen bereits existierender und gut publizierter Experimente.

Dies zeigte sich auch in der Planung und Konzeption der Experimente im Rahmen des interdisziplinären Projekts mit den wirtschaftswissenschaftlichen ForscherInnen. Die Erstellung und Ausformulierung der Instruktionen für die Treatmentvariationen erfolgte stets sehr gewissenhaft und in Abstimmung und Diskussion mit der Projektgruppe. Wie im Analyseteil sehr deutlich wurde, stellen die Instruktionen und die Art der Informationspräsentation aus der Perspektive der WirtschaftswissenschaftlerInnen einen zentralen Framingfaktor dar. Als Begründung für den hohen Stellenwert, der der gewissenhaften Erarbeitung der Instruktionen beigemessen wird, wurde auf die Anerkennung und Berücksichtigung der Ergebnisse von Kahneman und Tversky zu (semantischen) Framing-Effekten ${ }^{86}$ verwiesen.

In der letzten durchgeführten Experimentserie „Endowment-Effekt - USBStick“ orientierte man sich in der Vorbereitung und Durchführung des Kontrollbzw. Baseline-Treatments stark an einem anerkannten Experiment der beiden verhaltensökonomischen ForscherInnen Charles Plott und Kathryn Zeiler (Plott \&

\footnotetext{
${ }^{86}$ Eine ausführliche Darstellung dieses Aspekts findet sich im Analyseteil der Außenstruktur (5.2.2.1).
} 
Zeiler 2005). ${ }^{87}$ Plott und Zeiler hatten im Zuge ihrer Publikation sowohl die schriftlichen Instruktionen als auch den Text der mündlichen Erklärung veröffentlicht, welche sie für die Durchführung ihrer Experimente genutzt hatten. Sowohl in den Instruktionen als auch der mündlichen Erklärung wurde anhand von Beispielen versucht, den ProbandInnen zu erklären ${ }^{88}$, was die „beste Strategie“ sei, um in den nachfolgenden Spielrunden den Kauf- oder Verkaufspreis von Lotterielosen festzulegen. Zugleich wurden sie darüber informiert, welcher Mechanismus eingesetzt werden würde, um über den erfolgreichen Kauf oder Verkauf von Lotterielosen zu bestimmen.

Im Zuge der Vorbereitung der im Rahmen des Projekts durchgeführten Experimente erfolgte eine nahezu wörtliche Übersetzung dieser Dokumente von Plott und Zeiler (2005) aus dem Englischen ins Deutsche. Wie an anderer Stelle beschrieben ${ }^{89}$, führte ich in dieser Serie von Experimenten 18 der 19 erhobenen Sessions als Experimentatorin im Labor durch. ${ }^{90}$ Dabei wurden die einleitenden Informationen zur Erklärung des im Experiment verwendeten BDM-Mechanismus stets von mir präsentiert. In der teilnehmenden Beobachtung anderer Experimente hatte ich zudem weitere Varianten der Informationsdarstellung und der Unterstützung des Text- und Regelverständnisses der ProbandInnen beobachtet. Da quantitative Experimente, orientiert am Credo des Konstanthaltens der Randbedingungen, eine möglichst vollständige Standardisierung der Erhebungsbedingungen anstreben, konnte in den unterschiedlichen Serien von Experimenten jeweils nur eine Art der Variation beobachtet werden. Diese inhaltlichen Aspekte wurden aufgegriffen und für eine Reihe von Variationen im Sinne des qualitativen Experiments genutzt. Die vier durchgeführten Treatmentvariationen und die in ihnen vorgenommenen aktiven Eingriffe können in besonderem Maße als gegenstandsadäquat betrachtet werden. Denn diese basieren auf ähnlichen Ausprägungen der Informationspräsentation, die ich in unterschiedlichen Experimenten beobachtet hatte. Die Technik, die für die Hervorbringung der

\footnotetext{
${ }^{87}$ Im Unterschied zum Experiment von Plott und Zeiler (2005) wurden zwei weitere Lotterierunden hinzugefügt. Es handelte sich dabei um eine Kauf- und eine Verkaufsentscheidung. In beiden Fällen waren die Ergebnisse A und B, welche beim Ausspielen der Lotterie hätten eintreten können, identisch.

${ }^{88}$ Es handelte sich hier um den BDM-Mechanismus. Den ProbandInnen gegenüber wurde der Mechanismus nicht als BDM-Mechanismus vorgestellt, sondern als „Strategie“, die sie bei der Bestimmung des Kauf- bzw. Verkaufspreises für die Lotterielose anwenden sollten.

${ }^{89}$ Siehe dazu Abschnitt 4.1.1. Hier wurde ausgeführt, wie sich meine Feldrolle im Verlauf der Projektarbeit änderte.

${ }^{90}$ Stets war dabei mindestens ein weiteres Mitglied des Laborteams anwesend, in der Mehrzahl der Erhebungen auch mein Projektkollege.
} 
Variationen eingesetzt wurde, kann im Sinne Kleinings als Reduktion oder Abschwächung verstanden werden. Die Informationsvermittlung durch die Instruktionen vor Beginn der computergestützten Datenerhebung wurde unterschiedlich intensiv gestaltet.

Alle vier Experimente wurden von mir zusammen mit einem geschulten Labormitarbeiter durchgeführt. Die zusätzliche mündliche Erklärung (in Variation 1 und 2) erfolgte, wie in der Experimentserie „Endowment-Effekt USB-Stick“, auf der Grundlage der übersetzten Textvorlage von Plott und Zeiler (2005). Auch die Darstellung der Entscheidungssituationen, die die Teilnehmenden am Computer bewerten mussten, änderte sich in den vier Variationen nicht. Die Programmierung der Bildschirmoberflächen, die den ProbandInnen präsentiert wurden und mit denen sie interagieren sollten, wurden in gleicher Weise im Baseline Treatments, der oben beschriebenen Experimentserie, genutzt. $^{91}$

Die Methoden, die von mir zur Datenerhebung in den Sessions der qualitativen Experimente eingesetzt wurden, blieben in allen vier Fällen gleich. Es wurden Videodaten mittels einer Deckenkamera erhoben und zugleich an jeweils vier Arbeitsplätzen ${ }^{92}$ Aufnahmen von den ProbandInnen mittels Webcams gemacht. ${ }^{93}$ Direkt nach den Experimenten wurden mit jeweils sechs ProbandInnen pro Experiment leitfadengestützte Interviews geführt. Die Interviews wurden in drei zeitlich parallel verlaufenden Zweiergruppen durchgeführt und mittels Diktiergeräten aufgezeichnet. Eine Zweiergruppe interviewte ich stets selbst. Um potenzielle Interviewereffekte zu minimieren, wurden die beiden anderen Interviews jeweils von zwei weiteren SoziologInnen geführt. Es wurde dabei, wie im Methodenteil ausgeführt, der Leitfaden LF3c verwendet. Ziel der Datenerhebung war es, auf dieser Grundlage zu einer dichten Beschreibung der jeweiligen Variation zu gelangen. Diese Beschreibungen der unterschiedlichen Variationen des Phänomens wurden im Nachhinein miteinander verglichen und in Beziehung gesetzt, um genauere Kenntnis über die ,innere Struktur ‘ des Forschungsgegenstands zu erhalten. Die vier Variationen sollen im Folgenden kurz dargestellt werden:

\footnotetext{
${ }^{91}$ Beispiele dieser Bildschirmoberflächen wurden im Abschnitt der technischen Realisierung thematisiert. Es handelt sich um die Benutzeroberflächen, anhand derer die ProbandInnen gebeten werden, einen Preis für den Kauf bzw. Verkauf eines Lotterietickets einzutragen.

${ }^{92}$ Es handelte sich um die Arbeitsplätze in den Kabinen 18, 19, 22 und 23 im Mittelblock des Laborraums. Die Webcams wurden an den Bildschirmen der ProbandInnen befestigt.

${ }^{93}$ Die Experimente beinhalteten zudem einen Fragebogen, den alle ProbandInnen im Labor am Ende des Experiments ausfüllten. Neben soziodemographischen Daten der ProbandInnen wurden neun offenen Fragen zum Experiment gestellt. Dies entspricht dem Vorgehen im Baseline Treatment (Kontrollgruppe) des Experiments, welches hier als Orientierung diente. Die Daten der Fragebögen wurden in die vorliegende Auswertung nicht einbezogen.
} 

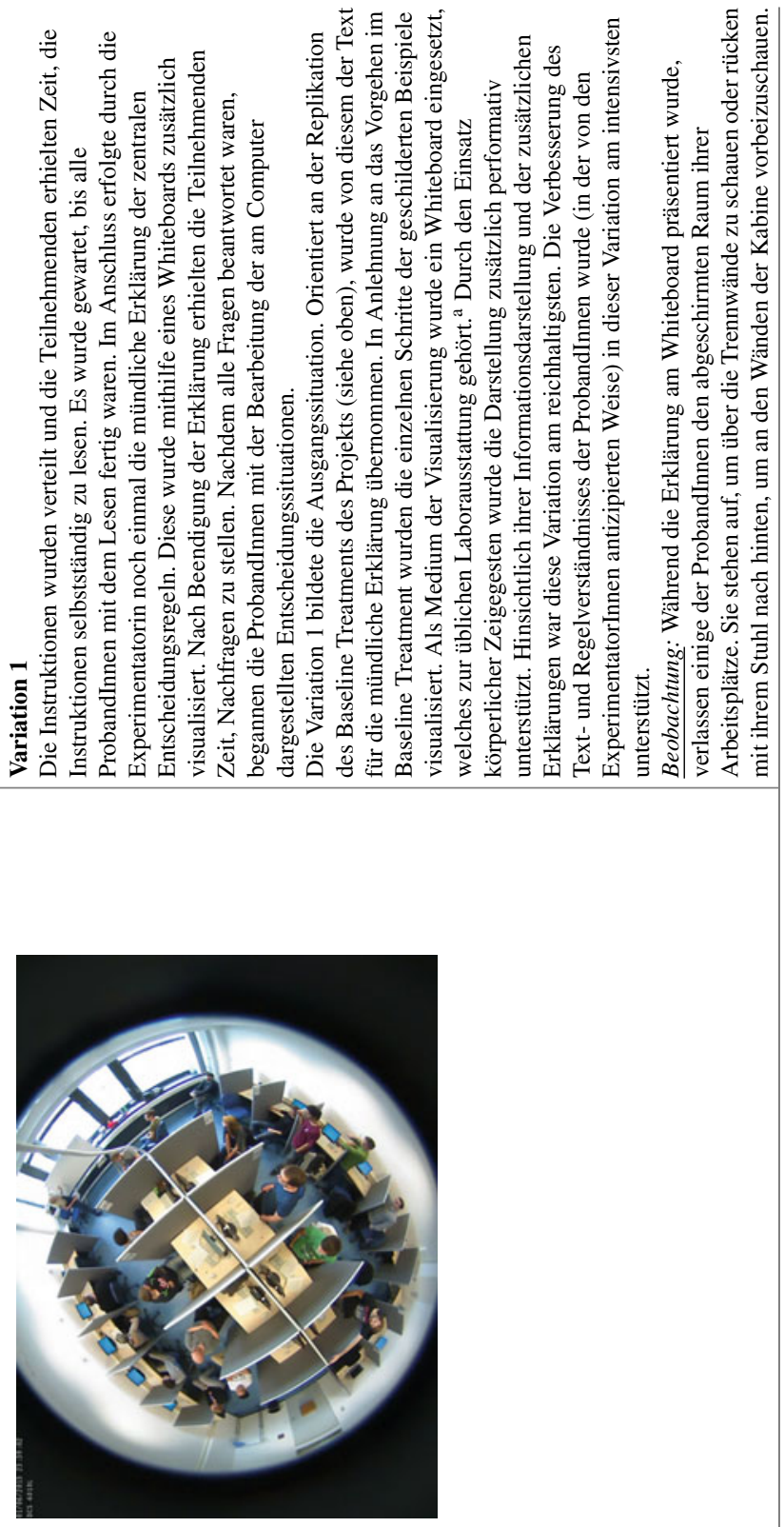

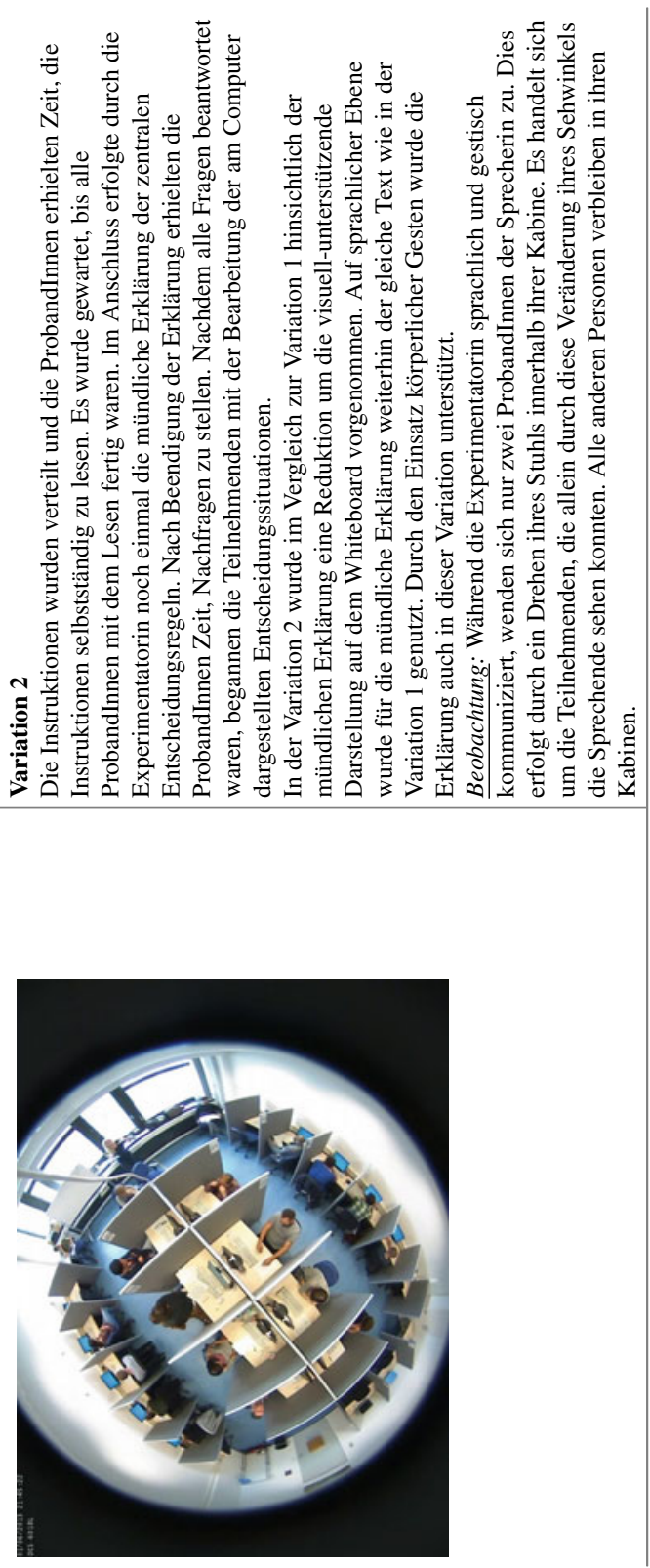


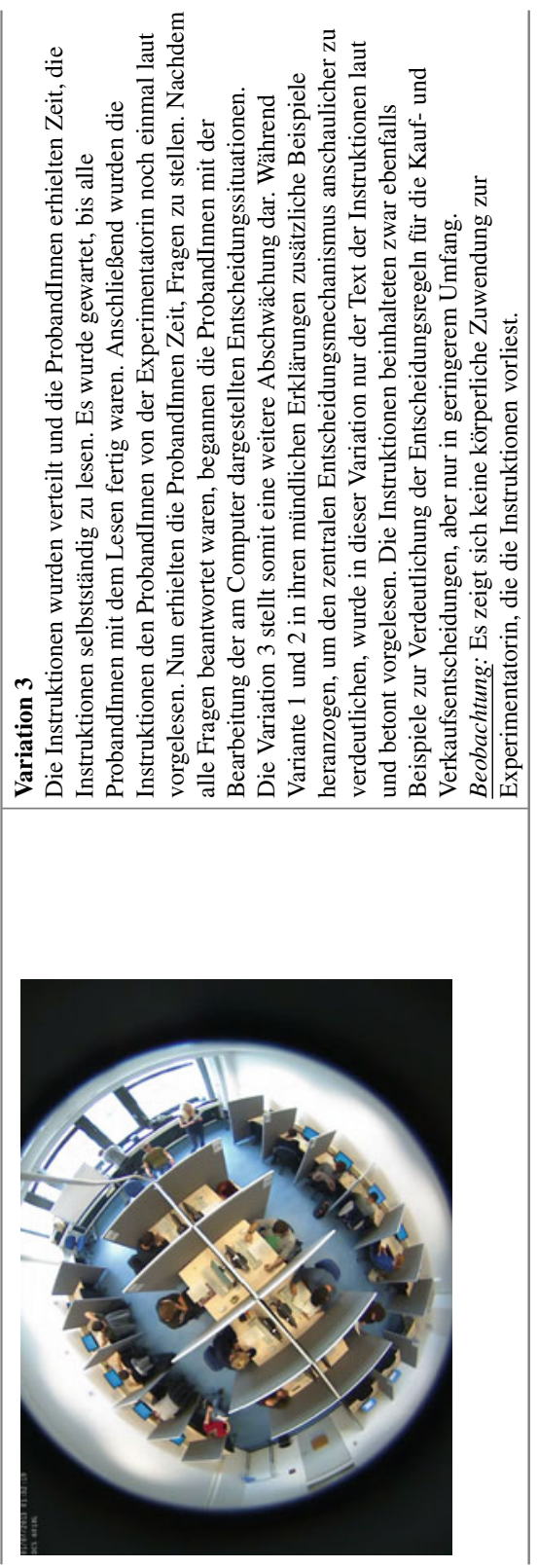




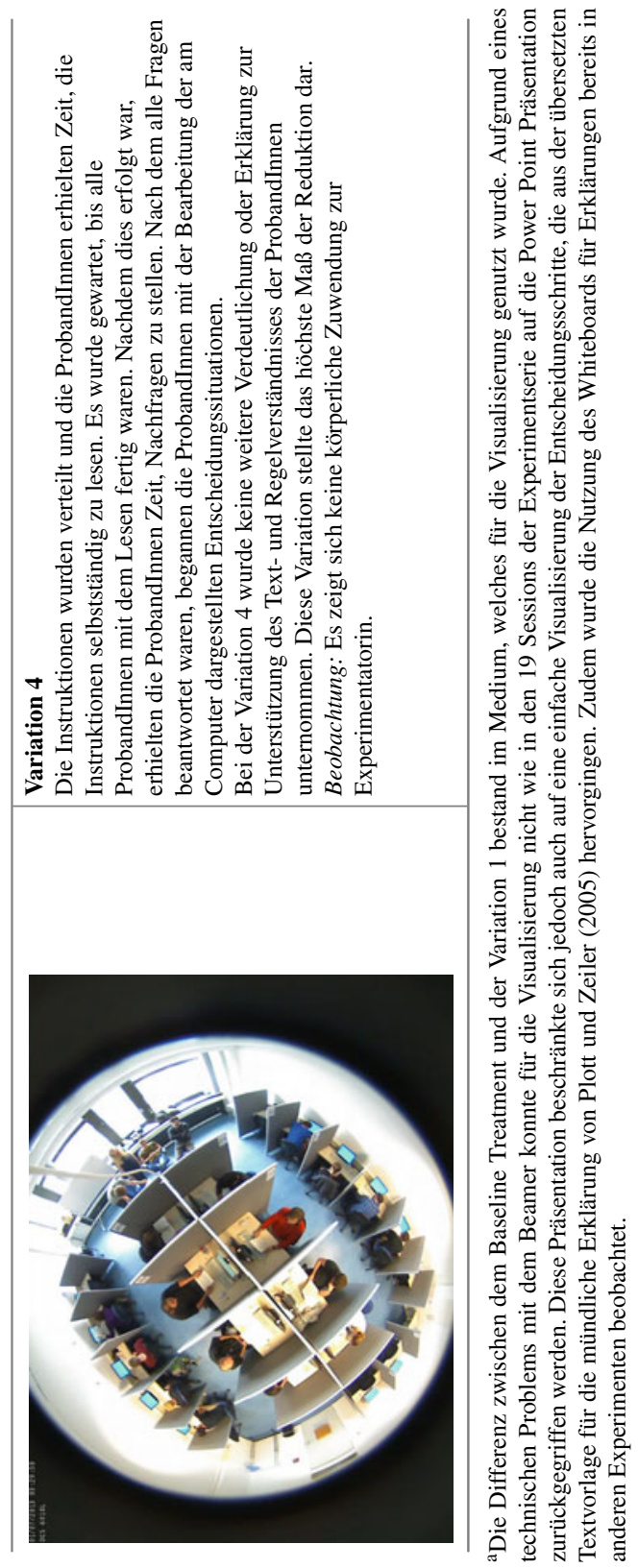


Es zeigt sich, dass eine reine Beschreibung der vier Variationen des Forschungsgegenstands nicht genügt, um die im Zuge der Gattungsanalyse herausgearbeiteten Merkmale des ökonomischen Laboratops zu valorisieren. Dieses Vorgehen ermöglicht es jedoch, gezielt strukturelle Variationen zu beobachten. Im Folgenden werden nun einige Analyseergebnisse dargestellt, die sich aus diesen Variationen ziehen ließen. Die Triangulation der Beobachtungsdaten mit den Interviews bleibt weiterhin von zentralem Stellenwert. In der Kombination mit den Interviews zeigte sich ein interessanter Effekt. Hinsichtlich des Stellenwerts der Instruktionen lässt sich auf Grundlage der vier durchgeführten Variationen eine gewisse Validierung einiger Erkenntnisse der Gattungsanalyse erzielen.

Wie im Abschnitt der Binnenstruktur dargestellt, ist es ein typisches Merkmal von Laborexperimenten, dass die ProbandInnen vor Beginn der Datenerhebung schriftliche Instruktionen erhalten. Die Instruktionen verwenden einfache Darstellungen der Spielsituation, die aus Sicht der Forschenden die relevanten Dimensionen und Aspekte erläutern. Im Zentrum der schriftlichen Erklärung stehen dabei die unterschiedlichen Rollen der ProbandInnen im Experiment, die Gewinnmodalitäten und zentralen Verhaltensregeln. Mit Hilfe von Beispielen im Text der Instruktionen, der Durchführung von Quizzen oder dem betonten, lauten Vorlesen des Instruktionstextes wird in vielen Experimenten versucht, das angestrebte Text- und Regelverständnis der ProbandInnen zu überprüfen und zu verbessern. Aus ökonomischer Perspektive wird davon ausgegangen, dass mittels der Instruktionen gezielt eine Rahmung für die Spielsituationen des Experiments induziert werden kann. In Anerkennung der Ergebnisse der Experimente von Kahneman und Tversky sind die Forschenden sensibilisiert gegenüber Rahmungseffekten, die auf semantischen Veränderungen des Instruktionstextes beruhen.

Auf der Ebene der Binnenstruktur zeigten sich zugleich Verbindungen von typischen verbalen und schriftlichen Elementen in einer geplanten sequentiellen Abfolge. Es handelt sich somit um eine multimodale Kommunikationssituation. Die zulässigen Arten der Interaktion sind in den unterschiedlichen Handlungsphasen dabei jeweils klar strukturiert und reglementiert. Im Zuge der Ergebnisdarstellung der Gattungsanalyse wurde aufgezeigt, dass die unterschiedlichen verwendeten Medien auch verschiedene Prägkräfte auf die kommunikativen Handlungen ausüben. Die Prägkräfte der Medien sind dabei unterschiedlich stark auf eine Übernahme der antizipierten Sprach- und Verhaltensspiele der Forschenden ausgerichtet. Die Engführung auf das Sprach- und Verhaltensspiel der Forschenden erfolgt dabei schrittweise.

Die Instruktionen sind ein interessantes Beispiel für die unterschiedlichen Modalitäten der Kommunikationssituation im Labor. Es zeigt sich im Labor ein Wechsel zwischen den unterschiedlichen Prägkräften, die ein Medium in 
der Vermittlung kommunikativer Inhalte ausübt. Hinsichtlich der Modalität der Kommunikation liegen sowohl am Bildschirm als auch in den Instruktionen die Inhalte primär in textlicher Form vor. Die Engführung auf das Sprachspiel und somit den Code der Forschenden ist im Fall der aktiven Bildschirmoberflächen jedoch deutlich gesteigert. Die detailreichen Beispiele zur technischen Realisierung im Analyseabschnitt der situativen Realisierung legten dar, wie der Handlungsspielraum der ProbandInnen durch die Festlegung von Parametern für zulässige Eingaben deutlich begrenzt wird.

Die Erkenntnisse, die sich aus den Erhebungen des qualitativen Experiments gewinnen lassen, bestätigen dieses Ergebnis der Gattungsanalyse. Trotz der Tatsache, dass den Teilnehmenden in den durchgeführten Variationen 3 und 4 des qualitativen Experiments weit weniger zusätzliche Erklärungen des Entscheidungsmechanismus geboten wurden, nahm die Anzahl der gestellten Frage oder auftauchenden Probleme im Experiment nicht markant zu. Die in den Variationen 3 und 4 vorgenommenen Reduktionen hinsichtlich der eingesetzten Techniken zur Unterstützung des Text- und Regelverständnisses führt in der Triangulation der Methoden zu aufschlussreichen Ergebnissen, die zugleich als Bestätigung der Ergebnisse der Gattungsanalyse interpretiert werden können. Im Rahmen des Leitfadeninterviews (LF3b) wurden die ProbandInnen zu ihrem Verständnis der Regeln im Experiment und zur Verständlichkeit der Instruktionen befragt. Es zeigt sich, dass die Benutzeroberflächen und die Instruktionen am Bildschirm für das praktische Handeln und die situative Rahmenbildung der ProbandInnen von zentraler Bedeutung sind. Die nachfolgenden exemplarischen Interviewausschnitte illustrieren diesen Aspekt:

Interviewausschnitt 1 (aus Variation 1):

Interviewerin1: Sehr schön. Und würdet Ihr sagen, waren die Regeln verständlich von dem Experiment?

Heidi: $\quad J a$, ich würde schon sagen, also da, wo-also bei mir ist es so, dass, ja klar, erstmal die ganzen Instruktionen, das 


\begin{tabular}{|c|c|}
\hline & $\begin{array}{l}\text { ist ja, erstmal kommt ein Standardtext von Euch }{ }^{94} \text {, dann } \\
\text { währenddessen lese ich mir in der Regel schon durch, was } \\
\text { genau zu tun ist. Der nächste Schritt in meinem Kopf ist } \\
\text { dann, wirklich zu verstehen, wie das Experiment abläuft } \\
\text { und mir dann ne Strategie festzulegen, möglichst vor dem } \\
\text { Experiment, in der Regel schaffe ich es nicht komplett, } \\
\text { sondern muss die ersten ein, zwei Versuche dann so ein } \\
\text { bisschen nach gut Glück machen und dann in der Regel ab } \\
\text { dem dritten habe ich dann meine Strategie, wie ich dann } \\
\text { verfahre. }\end{array}$ \\
\hline Isad: & $\begin{array}{l}\text { Ich glaube immer, das hängt auch an der Art des Expe- } \\
\text { rimentes ab. Also das Experiment an sich, wenn ich mir } \\
\text { die Anleitung durchlese, verstehe ich es meistens, denke } \\
\text { mir okay, es passiert, es wird irgendwie, ich weiß, was ich } \\
\text { machen soll, ich weiß, in was für einem Rahmen ich mich } \\
\text { bewegen soll, aber ganz den Durchblick kriege ich eigent- } \\
\text { lich immer erst, wenn das erste Mal gestartet ist, wenn } \\
\text { dann steht, ,Ach hier, ah, okay, Lose', ,Ach so, hm, jetzt } \\
\text { okay 'und dann sozusagen nach dem ersten, zweiten Mal, } \\
\text { dann weiß ich eigentlich immer ganz, genau, was los ist } \\
\text { und dann in Kombination mit den Anweisungen macht's } \\
\text { dann auch immer Sinn, ist eigentlich verständlich, aber } \\
\text { dass ich's jetzt wüsste nur durch's Lesen, was jetzt auf } \\
\text { mich zukommt, so halt= }\end{array}$ \\
\hline Heidi: & $\begin{array}{l}\text { Ja, man kriegt natürlich noch mal ne genauere Vorstellung, } \\
\text { wenn man den Bildschirm hat= }\end{array}$ \\
\hline Isad: & Genau. \\
\hline Heidi: & $\begin{array}{l}\text { Wenn man den Bildschirm hat, wenn man wirklich sieht, } \\
\text { welche Daten bekomme ich, beispielsweise jetzt hier mit } \\
\text { den Losen, mit den Gewinnchancen, dann ist es natürlich } \\
\text { noch mal übersichtlicher, aber an sich fand ich das Spiel } \\
\text { schon einfach muss ich sagen. }\end{array}$ \\
\hline
\end{tabular}

${ }^{94}$ Probandin Heidi nimmt hier Bezug auf die einleitende Begrüßung durch das Labormitglied 
Interviewausschnitt 2: (aus Variation 2)

Interviewerin1: Könnt Ihr bitte mal beschreiben, welche Schritte man so in einem Ablauf von dem Experiment wahrnimmt, also wie das so üblicherweise, was so das typische Experiment ausmacht? Gibt's das?

Mirco: $\quad J a$, ich würde schon sagen, also da, wo-also bei mir ist es so, dass, ja klar, erstmal die ganzen Instruktionen, das ist ja, erstmal kommt ein Standardtext von Euch, dann währenddessen lese ich mir in der Regel schon durch, was genau zu tun ist. Der nächste Schritt in meinem Kopf ist dann, wirklich zu verstehen, wie das Experiment abläuft und mir dann ne Strategie festzulegen, möglichst vor dem Experiment, in der Regel schaffe ich es nicht komplett, sondern muss die ersten ein, zwei Versuche dann so ein bisschen nach gut Glück machen und dann in der Regel ab dem dritten habe ich dann meine Strategie, wie ich dann verfahre.

Interviewerin 1: Okay.

Christian: $\quad$ Also bei mir ist es ähnlich, also ich lese mir die Instruktionen durch und dann, wenn man dann noch mal gemeinsam durchgeht, dann und eben dann, wenn dann diese Bildschirmoberfläche mit den Formularen dann aufgerufen wird, ja und dann meistens gehe ich dann auch vielfach intuitiv vor und probiere mich dann so =

Interviewerin1: So ein bisschen aus einfach durch, ja, okay?

Christian: Jagenau, genau.

Interviewausschnitt 3: (aus Variation 4)

Interviewer: $\quad$ Erst mal zu den Instruktionen: habt ihr die verstanden? Waren die klar, oder hat euch da was gefehlt?

D: $\quad$ Nö, die waren klar.

L: $\quad$ Das war eigentlich klar.

D: $\quad$ Also ich fand das gut mit dem Beispiel, da hat man das auch nen bisschen besser verstanden. 
I:

Hmhm, okay.

L:

Ja, das war eigentlich ziemlich. Na ja, die Unterscheidung, dass es eigentlich immer um die Lose geht und nur am Schluss um nen Gegenstand. Das musste ich zweimal lesen. Das hab ich nicht gleich gerafft. Aber in dem Moment, wo man's auf dem Bildschirm sieht, ist eigentlich alles klar.

Wie die Ausführungen der ProbandInnen in den Interviews zeigen, werden die Instruktionen als Orientierung für ein allgemeines Verständnis der Regeln der nachfolgenden Handlungen und Entscheidungen am Computer betrachtet. Es ist allerdings nicht immer gegeben, dass ein genaues Verständnis einzelner Begriff oder Aspekte beim Lesen der Instruktionen erreicht wird. Vielmehr verhilft den ProbandInnen das Lesen der Instruktionen dazu, einen Überblick über das nachfolgende Experiment zu erhalten und die grundlegenden Regeln des Spiels zu erfassen. Der ,Rahmen', in dem sich die nachfolgende Spielsituation entfaltet, wird damit abgesteckt. Die Instruktionen genügen jedoch nicht, um die vollständige Rahmenbildung zu vollziehen - erst die technische Realisierung durch die Benutzeroberflächen erlaubt dies. Sie baut die Brücke zwischen den in den Instruktionen formulierten Regeln und Erklärungen der Spielsituation und ihrer Umsetzung und Anwendung in den konkreten Entscheidungssituationen. Die visuelle Erfahrbarkeit bezieht sich jedoch nicht nur auf die Darstellung an sich, sondern auch auf die Konsequenzen, die die Handlungen der ProbandInnen mit sich bringen.

Den schriftlichen Instruktionen wird in der Konstruktion der Entscheidungssituation von den Forschenden ein zentraler Stellenwert für das Framing der Laborsituation beimessen. Die empirischen Beobachtungen und die Analyseergebnisse der Gattungsanalyse verweisen darüber hinaus jedoch auf die Relevanz der Multimodalität der Kommunikationssituation im Labor. Dies wurde insbesondere auch durch die Analysebeispiele auf der Ebene der situativen Realisierung ersichtlich. Diese stellten anschaulich dar, wie unterschiedliche Modalitäten kommunikativen Handelns in ihrem Zusammenspiel gezielt eingesetzt werden, um die situative Ordnung des Laborexperiments $\mathrm{zu}$ (re-)produzieren. Die ProbandInnen verweisen in beiden Interviewausschnitten deutlich darauf, dass man von den eigentlichen Spielsituationen erst einen klaren Eindruck erhält, wenn diese am Computerbildschirm präsentiert werden. Die schrittweise Engführung des Handlungsspielraums der ProbandInnen nimmt mit der Auseinandersetzung und im Zuge des Ausprobierens an den Bildschirmoberflächen zu. Durch die 
Ergebnisse der qualitativen Experimente lässt sich somit bestätigen, dass eine zunehmende Fokussierung auf das Sprach- und Verhaltensspiel der Ökonomen mittels der Bildschirmoberflächen erfolgt. Durch die Nutzung von speziellen Softwareprogrammen und die damit verbundene Standardisierung der präsentierten Inhalte wird so das Doing einer standardisierten Laborsituation unterstützt und das Experiment-Modul verankert. Durch die Prägkraft des technischen Mediums und die Begrenzung der Eingabemöglichkeiten wird zugleich zum Doing von rationalem und regelkonformem Verhalten beigetragen. Die Verankerung des Spiel(er)-Moduls und die Suche nach Spielregeln und Strategien beginnt bereits mit dem Lesen der Instruktionen. Doch die technisch-situative Realisierung ist ein notwendiger Schritt, um die Spielrahmung für die ProbandInnen auch durch die Konsequenzen ihres Handelns erlebbar zu machen. In den Interviews wurde von den ProbandInnen immer wieder auf das Ausprobieren oder auf ein intuitives Vorgehen in den ersten Spielrunden verwiesen. Es muss ausprobiert werden, wie das Experiment tatsächlich abläuft und wie ,richtig' gespielt wird. Zugleich erhalten die ProbandInnen durch die von ihnen getätigten Handlungen auch ein Feedback über die ihnen möglichen Eingaben und Handlungen. Erst in der Interaktion mit den Benutzeroberflächen vollzieht sich für die Handelnden somit das situative Sensemaking und damit die Rahmenbildung für die ihnen verbleibenden Handlungsspielräume. In der Auseinandersetzung mit den Bildschirmoberflächen zeigt sich daher nicht nur, welche Eingaben möglich sind, sondern auch, welche ,Strategie" man wählen muss, um Gewinne zu erzielen. Eben diese Gewinndimension verbindet wiederum das Spiel- mit dem Ökonomiemodul.

Im diesem letzten Abschnitt des Analyseteils wurde ein exemplarischer Versuch unternommen, um darzustellen, wie das Verfahren des qualitativen Experiments zur experimentellen Validierung der eigenen qualitativen Forschungsmethoden und -Ergebnisse der Gattungsanalyse eingesetzt werden kann. Es wurde dabei beschrieben, wie die experimentellen Eingriffe ausgewählt wurden. Der grundlegende Anspruch der qualitativen Forschung, lebensweltliche Zusammenhänge in natürlichen Situationen zu erforschen, und das Problem der Künstlichkeit einer Situation, die sich durch den Eingriff von ForscherInnen ergibt, scheinen nur schwer vereinbar. Im spezifischen Fall meines Forschungsfeldes des wirtschaftswissenschaftlichen Labors ist die Künstlichkeit der Situation jedoch ein zentrales und naturwüchsiges Element des Phänomens selbst. Die Herstellung von Variationen des Forschungsgegenstands durch einen aktiven Eingriff erscheint den ProbandInnen aus diesem Grund nicht als ungewöhnlich und irritierend, da es im Rahmen der Experimente üblich ist, dass sie sich mit verschiedenartigen Situationen auseinandersetzen müssen. Die vier Variationen der Experimente, 
die sich durch die Eingriffe ergeben, sind nicht beliebig, sondern in besonderem Maße gegenstandsadäquat, da sie aus einer intensiven Auseinandersetzung mit dem Feld und dem gängigen Vorgehen der LaborforscherInnen entstanden sind. Die hier dargestellten Variationen verfolgten nicht das Ziel, alle Ergebnisse der Gattungsanalyse zu prüfen. Sie zielten primär darauf ab, zu ermitteln, auf welche Weise das qualitative Experiment in der qualitativen Forschung gewinnbringend eingesetzt werden kann. Das qualitative Experiment erweist sich hier als eine gegenstandsadäquate Methode für die qualitative Erforschung einer experimentellen Laborsituation und bereichert die Analyse über den Prozess der Rahmenbildung im Labor. Basierend auf den vier oben dargestellten Variationen konnten einige Ergebnisse des Analyseteils, die das Zusammenspiel von Instruktionen und Bildschirmoberflächen betreffen, einem experimentellen Validitätstest unterzogen werden. Auch die ProbandInnen nehmen diese Typik als Teil der sozialen Veranstaltung des Laborexperiments wahr. Während die Instruktionen zu einer allgemeinen Orientierung in der Spielsituation verhelfen, erfolgt die weitere Ausbildung und Prüfung von Handlungsstrategien im Zuge der Auseinandersetzung mit den Bildschirmoberflächen. Die schrittweise Verengung auf die Sprachund Verhaltensspiele der Forschenden nimmt dabei von den Instruktionen zu den Bildschirmoberflächen deutlich zu. Es zeigte sich eine Robustheit der Ergebnisse der Gattungsanalyse über die vier Variationen hinweg.

\subsection{Zusammenfassung der Analyseergebnisse}

Die kommunikative Konstruktion des ökonomischen Laboratops wurde als der Prozess betrachtet, innerhalb dessen sich die Rahmenbildung im Labor vollzieht. Die soziale Situation des wirtschaftswissenschaftlichen Laborexperiments besteht jedoch nicht in einer, schlichten " sprachlichen Formulierung eines ökonomischen Rahmens, in welchem die folgenden Handlungen und Entscheidungen der ProbandInnen auszuführen sind. Wie in der Darstellung der Analyseergebnisse deutlich wurde, handelt es sich vielmehr um die gezielte Transformation des Alltags, in der drei zentrale Moduln zusammenspielen und unterschiedlich ineinander verschachtelt sind. Der Prozess der Rahmenbildung zeichnet sich dadurch aus, dass die ExperimentatorInnen in der Konzeption und Durchführung der Experimente gezielt ganz verschiedene Formen kommunikativer Handlungen einsetzen, um den von ihnen angestrebten Rahmen in der Situation des Experiments zu induzieren und zu verankern. 
Das nachfolgende Kapitel fasst zentrale Erkenntnisse zusammen, die in der Auseinandersetzung mit dem Forschungsgegenstand des Rahmenbildungsprozesses im ökonomischen Laborexperiment gewonnen werden konnten. Wie in dieser Arbeit deutlich wurde, zeichnete sich der Forschungsprozess dieser Arbeit durch ein fruchtbares Wechselspiel methodischer und theoretisch-konzeptioneller Auseinandersetzungen mit dem Forschungsgegenstand aus. Auch im abschließenden Resümee soll diesen beiden Dimensionen Rechnung getragen werden. Dieses Kapitel ist aus diesem Grund in zwei Abschnitte geteilt. In einem ersten Schritt werden die methodischen und konzeptionellen Erkenntnisse vorgestellt, die aus der Suche und dem Finden der geeigneten Methode hervorgingen. Den Fokus bildet hier das genutzte Analyseverfahren, welches als ,erweiterte Gattungsanalyse " bezeichnet wurde. In einem zweiten Schritt werden schließlich die inhaltlichen Ergebnisse der Analyse resümiert. Es werden hier nicht alle Analyseergebnisse wiedergegeben. Dieser Abschnitt bündelt vielmehr die Ergebnisse der unterschiedlichen konzeptionellen Zugänge und Zugriffe, die für die Bearbeitung der Fragestellung genutzt wurden. Er bildet eine Synthese, welche die abschließende Beantwortung der Forschungsfrage ermöglicht.

\subsubsection{Konzeptionelle und methodische Erkenntnisse}

Dem empirischen Programm des kommunikativen Konstruktivismus folgend, ist auch die vorliegende Arbeit von dem Anspruch geprägt, dem Spannungsverhältnis zwischen Ethnomethodologie und der systematischen Anerkennung und Einbeziehung des institutionellen Kontexts des Handelns Rechnung zu tragen (Knoblauch 2017: 53). Der Wert, der der Gattungsanalyse im Rahmen dieser Arbeit beigemessen wird, besteht darin, den Anspruch einer Verbindung von Ethnomethodologie und verfestigten übersituativen Kontextelementen anhand einer empirischen und datengeleiteten Analyse praktisch einzulösen. Die Gattungsanalyse wird hier als ein wissenssoziologisches Analyseverfahren genutzt und dient im Rahmen dieser Arbeit der praktischen Umsetzung des empirischen Programms des kommunikativen Konstruktivismus.

Die Anwendung der Gattungsanalyse als Analyseheuristik erlaubt es dabei, den institutionellen Kontext, der die kommunikativen Handlungen prägt, als integralen Bestandteil in die Analyse aufzunehmen. Die allgemeine inhaltliche Zielstellung der drei Analyseebenen wurde für die eigene Analyse beibehalten. Wie weiter unten dargestellt, bedingt jedoch die Spezifik des Forschungsgegenstands eine Abwandlung der dort thematisierten Inhalte gegenüber einer ,klassischen ' Gattungsanalyse (orientiert an Luckmann 1986), welche sich auf sprachliche und alltägliche Kommunikationsmuster fokussiert. 
Die Außenstruktur erfasste dabei den institutionellen Kontext des Handelns. Inhalt dieser Analyseebene sind solche Elemente, die die musterhafte Kommunikation von außen bestimmen, wie das spezifische soziale Milieu oder die soziale Situation, in welcher diese kommunikativen Handlungen typischerweise anzutreffen sind. Die Merkmale der Außenstruktur geben Auskunft über die milieuspezifischen Wissensbestände, aus welchen die indexikalen Wissenselemente entstammen, deren Aktualisierung sich auf der Ebene der Binnenstruktur und der situativen Realisierung zeigt. Die Analyseebene der Binnenstruktur fokussiert solche Merkmale des empirisch beobachtbaren Handlungsvollzugs, welche als typisch für die Hervorbringung des Phänomens betrachtet werden können und in ihrer Musterhaftigkeit nicht vom interaktiven und sequenziellen Handlungsvollzug geleitet sind. Als Merkmale der situativen Realisierungsebene wiederum wurden solche musterhaften kommunikativen Merkmale der Handlungssituation herausgestellt, die sich auf die sequenzielle Abfolge der Handlungen und ihre situative Koordination bezogen. Die Ebene der situativen Realisierung betont dabei, dass es sich bei den beobachteten Phänomenen um Interaktionsphänomene handelt und ihre situative Realisierung von entscheidender Relevanz für ein Verständnis der Kommunikationsmuster ist. Merkmale der Ebene der situativen Realisierung sind somit solche, die sich der interaktiven Bewältigung der Kontingenzen der spezifischen empirischen Situationen widmen.

Die Gattungsanalyse diente hier darüber hinaus als integratives Verfahren der Datentriangulation. Inspiriert durch das Vorgehen in der Studie zu den PowerPoint Präsentationen (Schnettler \& Knoblauch 2007) wurden auch in der vorliegenden Arbeit auf den drei unterschiedlichen Analyseebenen verschiedene Datensorten priorisiert für die Analyse herangezogen. Die vielfältigen Daten, die durch die intensive und langjährige Ethnographie gewonnen wurden, konnten so gewinnbringend mit den datenintensiven Videomaterialien der aufgezeichneten Laborexperimente kombiniert werden. Mittels der Gattungsanalyse konnte auf diese Weise eine systematische Datentriangulation erreicht werden. Die unterschiedlichen Datensorten zielten nicht auf eine gleichartige Beschreibung des untersuchten Phänomens aus verschiedenen Perspektiven, sondern bereicherten unterschiedliche Aspekte der Analyse. Die Gattungsanalyse erwies sich für den vorliegenden Forschungsgegenstand als ein besonders gegenstandsadäquates Analyseverfahren. Wie die Arbeit aufzeigte, sind ökonomische Laborexperimente durch vielfältige Standardisierungsbemühungen und durch eine starke Orientierung an methodischen Prinzipien gekennzeichnet. Die Berücksichtigung dieser und weiterer institutioneller Kontextelemente als integraler Bestandteil der Analyse ist für die Beantwortung der Forschungsfrage von enormem Wert. Mit der Anwendung der Gattungsanalyse im Rahmen meiner Arbeit gehen jedoch gewisse 
Überschreitungen des klassischen konzeptionellen Rahmens und des Gegenstandsbereichs einher. Diese bieten Anstöße für mögliche Weiterentwicklungen des konzeptionellen Rahmens sowie des Gegenstandsbereichs der kommunikativen Gattungsanalyse.

Die Ausweitung der Außenstruktur - zur adäquaten Betrachtung eines wissenschaftlichen Kommunikationsphänomens

Kommunikative Gattungen betrachten typische kommunikative Handlungsvollzüge, in denen soziale Wirklichkeit vermittelt und erzeugt wird. Sie zielen dabei darauf ab, die Struktur zu bestimmen, mittels derer Wissensbestände mit unterschiedlichem Expliziertheitsgrad vermittelt werden. Kommunikative Gattungen bilden somit ,verfestigte Formen ihrer Vermittlung“ (Luckmann 1986: 196). Während die Analyse kommunikativer Gattungen musterhafte Formen alltäglicher Kommunikation untersucht, handelt es sich bei meinem Forschungsgegenstand um ein kommunikatives Phänomen aus dem Bereich der Wissenschaft. Diese Differenzierung soll nicht darauf verweisen, dass die Struktur kommunikativer Handlungen von Alltagsmenschen und WissenschaftlerInnen grundlegend unterschiedlich ist. Ganz im Gegenteil. Gerade die Laborstudien (Knorr-Cetina 1984; Knorr-Cetina \& Mulkay 1983; Latour \& Woolgar 1979) haben vielfältig aufgezeigt, dass sich die Muster der Hervorbringung von sozialer Wirklichkeit in beiden Sphären nicht maßgeblich unterscheiden. Die Wissensbestände, auf die die Akteure im Vollzug ihrer kommunikativen Handlungen indexikal verweisen, unterscheiden sich jedoch sehr wohl. Im Fall der ökonomischen LaborforscherInnen können sie nicht als Teil eines allgemeinen gesellschaftlichen Wissensvorrats betrachtet werden. Die aus der Perspektive des kommunikativen Konstruktivismus eingeforderte Berücksichtigung der institutionellen Seite des Handelns bedingt jedoch eine Intensivierung und Ausweitung der außenstrukturellen Analyseebene. Dies gilt sowohl für den Forschungsprozess als auch für die Darstellung dieser Analyseebene. Für ein Verständnis der typischen kommunikativen Handlungen, die im Vollzug der ökonomischen Laborexperiments beobachtet werden können, ist dieses Wissen und seine Explizierung von entscheidender Bedeutung. Auf diese Weise wird es möglich, das Ziel oder die Funktion, die den musterhaften Formen kommunikativer Handlungen beigemessen wird, zu verstehen und zu offenbaren. Die Ebene der Außenstruktur wurde aufgrund dessen deutlich vertieft und ausgebaut.

Orientiert am Vorgehen der Videographie wurde die Analyse von Interaktionssituationen mit einer soziologischen Ethnographie verwoben. Die mehrjährige Dauer und Intensität der Ethnographie überstieg dabei deutlich die gängige Anwendung der fokussierten Ethnographie (Knoblauch 2001). Die Fokussierung, 
die im eigenen Forschungsprozess vorgenommen wurde, bezog sich vielmehr auf die gezielte Auseinandersetzung mit den Techniken und Kompetenzen der Handelnden, die zur Rahmenbildung im Labor beitragen. Es wurde dabei versucht, dem ethnomethodologischen Anspruch der Aneignung des „Mitgliederwissens“ (Ten Have 2002) nachzukommen und diesen als reflexive Grundlage der institutionellen Einbettung auch in der Analyseebene der Außenstruktur darzustellen. Die Analyseebene der Außenstruktur stellt aus diesem Grund die paradigmatischen Grundlagen dar, an denen sich die Forschenden bei der Durchführung der ökonomischen Laborexperimente typischerweise orientieren. Das Hinzuziehen ethnographischer Informationen wurde nicht nur verwendet, um die allgemeine institutionelle Einbettung des kommunikativen Ereignisses auszuweisen, sondern auch, um das ,Warum“ der typisch genutzten Ethnomethoden und kommunikativen Muster (des, Wie') in der analytischen Darstellung explizieren zu können.

\section{Erweiterung der Ebene der Binnenstruktur zur Integration materieller Objek- tivationen}

Wie oben ausgeführt, bilden musterhafte Formen der sprachlichen Kommunikation den primären Gegenstand klassischer kommunikativer Gattungsanalysen. Kommunikatives Handeln wurde dabei im Anschluss an Luckmann (1986) als eine besondere Art sozialen Handelns aufgefasst. Die soziale Situation und ihre materiellen Elemente ebenso wie Aspekte der körperlichen Performanz wurden in dieser Konzeption der Ebene der situativen Realisierung zugerechnet. Die Zuordnung zu dieser Ebene bedingt, dass diesen Aspekten ,nur' eine handlungskoordinative Wirkung zugesprochen wurde. Der Status eines binnenstrukturellen Merkmals, das nicht auf die Koordination des sequentiellen Handlungsablaufs gerichtet ist, wurde ihnen damit jedoch abgesprochen. Am Beispiel von PowerPoint-Präsentationen (Knoblauch \& Schnettler 2007) wurde aufgezeigt, wie fruchtbar die Öffnung der Gattungsanalyse für die körperlichen Akte des Zeigens ist. In meiner Studie wird ein ähnliches Ziel verfolgt. Anhand des eigenen Forschungsbeispiels geht es um eine Öffnung des Konzepts für materielle Aspekte der Handlungssituation. Die Analyse verdeutlichte, dass körperliche Präsenz, materielle Elemente und die strukturierte Einbindung technischer Medien zentrale Merkmale der typischen kommunikativen Handlungen im Vollzug des Laborexperiments bilden.

Im Anschluss an den kommunikativen Konstruktivismus wird in dieser Arbeit davon ausgegangen, dass auch Objekte kommunikative Handlungen vollziehen können. Wie in Knoblauch $(2013,2017)$ ausgeführt, tragen auch diese Aspekte 
maßgeblich zur kommunikativen Konstruktion der geteilten Wirklichkeit der Handelnden bei. Objekte, die sich in der Interaktion als bedeutsam erweisen, können dabei als Verdinglichungen (,Thingification“ (Barad 2003)) von Wissenselementen betrachtet werden. Sie bilden Objektivationen kommunikativer Handlungen und der damit verbundenen Wissensbestände, welche in der Interaktionssituation durch das Handeln der Interagierenden aktualisiert werden. Die Erweiterung des Kommunikationsbegriffs bzw. des kommunikativen Handelns, die der kommunikative Konstruktivismus vornimmt, bietet dabei die Chance, auch die Gattungsanalyse als wissenssoziologische Methode auf einen erweiterten empirischen Gegenstand anzuwenden. Übernimmt man diesen tiefer angelegten Kommunikationsbegriff für die Suche nach typischen Mustern kommunikativen Handelns, so können auch materielle und technische Aspekte als integrale Bestandteile der typischen und musterhaften kommunikativen Lösungen von Handlungsproblemen berücksichtigt werden. Dies erwies sich in meiner eigenen Arbeit als sehr fruchtbar und verweist zugleich auf das Potenzial dieser Betrachtungsweise für andere Interaktionssituationen, die ebenfalls durch ein charakteristisches Zusammenspiel materieller, technischer, körperlicher und sprachlicher Formen kommunikativen Handelns geprägt sind. Die Nutzung von Videodaten zur Erfassung und Analyse dieser Interaktionselemente erwies sich dabei als höchst vorteilhaft.

Wie die Analyse aufzeigte, sind materielle Aspekte in der sozialen Situation des ökonomischen Laborexperiments von solch prägender Relevanz, dass sie selbst einen zentralen Teil der kommunikativen Konstruktion des Phänomens bilden. Es sind dabei unterschiedliche Arten von materiellen Objekten und Objektivationen zu unterschieden. Zwei wichtige Aspekte sollen nachfolgend kurz am Beispiel des Laborraums und des Einsatzes der Spielchips verdeutlicht werden.

Im Fall des beobachteten Experimentallabors sind die Trennwände, die Nummerierung und die grundlegende Ausstattung der Arbeitskabinen im Labor vorhanden, unabhängig davon, ob sich Personen im Labor befinden oder nicht. Zentrale Aspekte der Raumstruktur können somit als ,permanent' bezeichnet werden. Aus der Perspektive des kommunikativen Konstruktivismus besteht die zentrale Frage jedoch stets darin, welche Bedeutung Objekten im Vollzug kommunikativer Handlungen beigemessen wird, wie und in welcher Form sie also handlungswirksam werden. Durch den Einsatz materieller Elemente wird bereits vor der Aktualisierung des Handlungsvollzugs bestimmt, wo im Laborraum gesessen, gelaufen, gearbeitet und überwacht werden soll - und dies unabhängig vom Vollzug einzelner Handlungssituationen. Zugleich wird der Möglichkeitsspielraum für Handlungen begrenzt. Da, wo die Trennwände stehen, kann nicht gelaufen werden. Wo es eine Wand gibt, kann nicht einfach unauffällig zum Nachbarn geschaut werden. Durch das gebaute permanente materielle Setting 
werden die typisch ablaufenden Interaktionen in ihrem Vollzug beeinflusst. Die permanenten materiellen Aspekte der Raumstruktur werden aus diesem Grund der Ebene der Binnenstruktur der Gattungsanalyse zugeordnet. Gerade im Fall des Experimentallabors erfüllen die eingesetzten materiellen Elemente gezielt kommunikative Funktionen. Die Raumstruktur des Labors bildet einen zentralen Anteil an der Standardisierung. Die potenzielle Kontingenz von Rahmenbedingungen und -interpretationen, die mit körperlichen und verbalen Formen kommunikativer Handlungen einhergehen, werden hier durch den Einsatz materieller und technisch vermittelter Objektivationen so weit wie möglich zu reduzieren versucht.

Am Beispiel des Einsatzes von Spielchips lässt sich eine weitere Dimension der gezielten Nutzung materieller und technischer Objektivationen thematisieren. Vor dem Betreten des Labors zieht jeder der Eintretenden einen nummerierten Spielchip. Die Nummer des Spielchips weist den ProbandInnen einen Arbeitsplatz im Labor zu. Als Zeichenträger kommunikativer Handlungen und abgelöst von der Körperlichkeit der ExperimentatorInnen sind die Spielchips geeignet, das Problem sozialer Störfaktoren, wie Versuchsleitereffkete oder die Vermutung einer Manipulation oder Täuschung, in spezifischen Phasen im Prozess des Experimentierens zu verringern. Das Setting des Experimentallabors zielt hier, wie dargestellt, auf die Vereinzelung der ProbandInnen in ihren Arbeitskabinen ab, um ihre individuellen Entscheidungen in ökonomisch relevanten Situationen zu erheben. Die Qualität der materiellen Objektivation des Spielchips besteht dabei gerade in ihrer performativen ,Entkörperlichung`. Die Loslösung vom körperlichen Handeln des Labormitglieds ist hier der entscheidende Schritt, um die Zufälligkeit der Platzzuweisungen auch den ProbandInnen gegenüber zu verdeutlichen. Direkte Manipulation der Zuweisung einzelner Arbeitsplätze wird hier auf standardisierte Weise ausgeschlossen, da der Spielchip von den ProbandInnen selbst gezogen werden kann. Die Nummer des Spielchips wiederum weist den einzelnen ProbandInnen ihren konkreten Arbeitsplatz zu. Als binnenstrukturelles Merkmal ist der Spielchip hier eine Objektivation der randomisierten Zuweisung. Der von den ProbandInnen selbst erlebte Zufall ist eine Qualität der Objektivation. Das gleiche Moment zeigt sich im charakteristischen Einsatz von Würfeln, dem Ziehen von Karten oder Losen. Die in der Situation des Experiments standardisierten und randomisierten Aspekte werden durch die disziplinäre Perspektive der ExperimentalforscherInnen und der jeweiligen Peergroup oder Forschungscommunity bestimmt, für die sie ihre Ergebnisse publizieren möchten.

Im Unterschied zu körperlichen und verbalen Formen kommunikativer Handlungen besitzen materielle Objektivationen eine andere Art von Performativität, 
die durch die Permanenz und den Eigensinn begründet wird, die mit der Materialität ihrer Zeichenträger zusammenhängen. Kommunikatives Handeln als Wirkhandeln zu verstehen, ist eine der zentralen Grundannahmen des kommunikativen Konstruktivismus. Körperliche und verbale Formen kommunikativer Handlungen werden in ihren potenziellen oder faktischen situativen Realisierungen handlungswirksam. In ihrer Musterhaftigkeit können sie auf die Koordination der Handlungsabfolge gerichtet oder von dieser unabhängig sein. Je nach ihrer Spezifik und Verfestigung können sie analytisch der Ebene der Binnenstruktur oder situativen Realisierung zugeordnet werden. Die Durchführung kommunikativer Handlungen ist zwar an diesen Mustern orientiert, geht jedoch nicht in diesen auf. In konkreten Interaktionssituationen stellen sie dabei ,individuelle Variationen über ein Muster“ (Ayaß 2011: 281) dar. Ganz im ethnomethodologischen Sinne werden somit die ordnungsstiftenden Muster in der Interaktionssituation gleichzeitig produziert und reproduziert. Die Varianz materieller Objektivationen, die sich in der Interaktionssituation als wirksam erweisen, ist deutlich eingeschränkt. Gerade in Situationen, die sich um eine starke Standardisierung bemühen, werden materielle Elemente gezielt eingesetzt, um die Varianz kommunikativer Handlungen zu reduzieren. Es sollen hier gezielt gleichartige Situationen mit gleichen Randbedingungen geschaffen werden. Im Fall des Laborexperiments weist der geschilderte Einsatz von Objektivationen darauf hin, dass ihre Einordnung auf der Ebene der situativen Realisierung problematisch ist. In solchen Situationen können materielle Objektivationen vielfach passender der Ebene der Binnenstruktur zugeordnet werden. Gerade Situationen, in denen materielle Objekte wichtige Rahmungselemente bilden und entäußern, sollte ihre Einordnung auf der Ebene der situativen Realisierung kritisch hinterfragt werden.

Wie aufgezeigt wurde, erwies sich die Anwendung der Gattungsanalyse als Verfahren für die Analyse des Rahmenbildungsprozesses im ökonomischen Laboratop als sehr geeignet. Dies lag insbesondere daran, dass die drei Ebenen der Gattungsanalyse nicht nur eine theoretisch-konzeptionelle Synthese ermöglichten, sondern zugleich auch eine Chance für die Integration unterschiedlicher Datensorten eröffneten. Die Anwendung dieses Verfahrens motivierte in Auseinandersetzung mit dem untersuchten empirischen Gegenstand zu zwei Öffnungsprozessen des ,klassischen' konzeptionellen Vorgehens. Einerseits erwies sich die Erweiterung der Ebene der Außenstruktur für einen Forschungsgegenstand aus dem Bereich der Wissenschaft als fruchtbar. Auf diese Weise wurde es möglich, die beobachtbare Typizität kommunikativer Handlungen an die Wissensbestände rückzubinden, die eben diesen Legitimität, Notwendigkeit und Sinn verleihen. Ein zweiter Anstoß für eine Erweiterung der Gattungsanalyse ergab 
sich aus der Relevanz materieller Objektivationen für das beobachtete Kommunikationsphänomen. Dieser lässt die generelle Zuordnung materieller Aspekte zur Ebene der situativen Realisierung als problematisch erscheinen und regt zu einer gegenstandsangemessenen Einordnung zu den verschiedenen Strukturebenen der Gattungsanalyse an. Die vorliegende Arbeit versteht sich hier als ein Diskussionsanstoß, um den Anwendung der Gattungsanalyse als integratives Analyse- und Auswertungsverfahren zu erweitern.

\subsubsection{Inhaltliche Ergebnisse}

Das ökonomische Laboratop ist das wissenschaftliche Umfeld, welches zur Untersuchung des Verhaltens von rationalen Akteuren in ökonomisch relevanten Entscheidungssituationen genutzt wird. Aufbauend auf der dargestellten konzeptionellen Perspektive wurde der Prozess der Hervorbringung des Phänomens als kommunikative Konstruktion des ökonomischen Laboratops bezeichnet. Die hier vorgestellte Analyse hatte nicht zum Ziel, zu untersuchen, ob das Handeln der Akteure im Labor wissenschaftlichen Kriterien der soziologischen oder ökonomischen Rationalität entspricht. Sie wandte sich vielmehr der Frage zu, wie die spezifische Rahmung des wirtschaftswissenschaftlichen Laborexperiments hervorgebracht wird. Es wurde anhand der Analyse herausgestellt, wie durch die Handelnden schrittweise eine Situationsrahmung hervorgebracht wird, welche aus Sicht der Forschenden das Handeln der ProbandInnen als rationales Entscheidungsverhalten analysierbar macht. Der analytische Fokus war darauf ausgerichtet, zu untersuchen, wie die Dominanz eines Orientierungsrahmens situativ und zugleich in einer typischen Ordnungshaftigkeit erzeugt wird. Die vorliegende Arbeit zielte somit auf eine Analyse der performativen Erzeugung von Rahmungsrationalitäten, welche im Prozess des Experiments selbst auf beobachtbare Weise erzeugt, verankert, aufrechterhalten und durchgesetzt werden.

Im Sinne der Goffmanschen Terminologie sollte auf diese Weise differenziert betrachtet werden, welche Arten von Modulationen der sozialen Alltagswirklichkeit die Rahmenbildung im ökonomischen Laboratop prägen. Die Modulationen der Transformationsprozesse von Sinn können in empirischen Situationen beobachtet werden. Die Moduln, die als System von Konventionen die Wissensbasis der Modulationen bilden, treten in den empirischen Situationen selbst jedoch meist nur vermittelt in Erscheinung. Die analytische Perspektive dieser Arbeit zielte darauf $a b$, dem Sprung zwischen den Moduln und Modulationen in der Analyse Rechnung zu tragen. Orientiert an der Gattungsanalyse wurde dazu die Trennung in drei Analyseebenen übernommen. Die analytische Trennung 
in die Ebenen der Außen- und Binnenstruktur und der situativen Realisierung zielte dabei nicht darauf ab, das ökonomische Laborexperiment als kommunikative Gattung zu rekonstruieren. Vielmehr wurde das Verfahren angewandt, um die Bezugnahme und Verfestigung unterschiedlicher Wissensbestände und ihre Aktualisierung zu verdeutlichen.

Die Rahmenbildung ist ein sozialer Prozess, der in der Interaktion der Handelnden hervorgebracht wird. Modulationen, welche Sinn transformieren, zeigen sich in der empirischen Wirklichkeit. Die soziale Situation der Durchführung von Experimenten wurde aus diesem Grund als ein Doing konzeptualisiert. Diese Bezeichnung diente dazu, die prozesshafte Hervorbringung des Phänomens und die Gleichzeitigkeit von Produktion und Reproduktion sozialer Wirklichkeit zu unterstreichen.

Im Sinne einer Analyseheuristik wurden dabei verschiedene Ebenen dieses Doings unterschieden. Der Zweck dieses Vorgehens bestand darin, differenziert aufzuzeigen, welchen Anteil die unterschiedlichen typischen kommunikativen Handlungen und Muster an der empirisch beobachtbaren Hervorbringung des Untersuchungsphänomens besitzen. Die drei Ebenen des Doing bezeichnen somit drei Arten von Modulationen, die sich im Vollzug des ökonomischen Laborexperiments zeigen. Sie sind zugleich Ausdruck der jeweiligen Moduln, auf denen sie gründen. Die nachfolgende abschließende Synthese fasst nun zusammen, auf welche Elemente die drei Moduln maßgeblich Bezug nehmen und wie sie in ihrer spezifischen Verknüpfung zur Eigengesetzlichkeit des Phänomens des ökonomischen Laboratops beitragen.

\section{Experiment-Modul}

Experimente sind eine Form des wissenschaftlichen Forschungsdesigns. Das soziale Milieu des Laborexperiments ist das der Wissenschaft. Laborexperimente sind kein Selbstzweck, sondern zielen darauf ab, Daten für wissenschaftliche Untersuchungen zu erheben. Um als Laborexperimente gelten zu können, müssen sie spezifische wissenschaftliche Kriterien erfüllen. Auf eben diese Ansprüche nimmt das Experiment-Modul Bezug. In empirischen Situationen zeigt sich hier eine Bezugnahme auf zentrale Prämissen der experimentellen Forschung im Labor und auf die mit ihnen verbundenen Ansprüche. Zugleich wird durch die Beachtung und Umsetzung dieser Anforderungen der Anspruch erhoben, dass im Zuge von Laborexperimenten wissenschaftlich valide Daten produziert werden können. Zentrale Rahmungselemente dieser Modul-Ebene zeigen sich empirisch in den vielfältigen Standardisierungsbemühungen der Forschenden. Andere Elemente stellen Realsierungen von typischen und wissenschaftlich anerkannten Kontrolltechniken zur Ausschaltung von Störvariablen dar. Ausdruck finden diese 
Rahmungselemente in ganz unterschiedlichen empirisch beobachtbaren Formen kommunikativen Handelns und materieller Objektivationen. Diese Rahmungsaspekte wurden als Doings einer spezifischen Laborsituation bezeichnet. Um dem Anspruch des Konstanthaltens von Untersuchungsbedingungen über den Zeitraum eines einzelnen Experiments hinweg zu entsprechen, sind viele dieser Rahmungselemente binnenstrukturelle Merkmale der Rahmung des ökonomischen Laboratops.

Das Experiment-Modul kann auch als maßgeblicher Grund dafür betrachtet werden, dass den ProbandInnen gegenüber nicht formuliert wird, dass sie sich an den ökonomischen Aspekten der Spielsituation orientieren müssen. DemandEffekte könnten sonst wiederum selbstreferenziell als Störfaktor in der Situation wirken. Stattdessen werden Rahmungsrationalitäten induziert, die eine Orientierung an ökonomischen Dimensionen der Situationsdefinition als priorisierte Rahmungselemente nahelegen.

\section{Mikroökonomie-Modul}

Das experimentelle Forschungsdesign und die mit diesem verbundenen Ansprüche geben das Thema eines Experiments nicht vor. Wirtschaftswissenschaftliche Laborexperimente zielen auf die Untersuchung von Entscheidungsverhalten in wirtschaftlich relevanten Situationen. Das Mikroökonomie-Modul nimmt Bezug auf eben diesen Forschungsgegenstand. Auf der Ebene des Doings der spezifisch ökonomisch-rationalen Verhaltenserwartungen stehen die Methoden und Praktiken im Fokus, die die Forschenden einsetzen, um den ProbandInnen eine Situationsdefinition nahezulegen, welche an ökonomisch relevanten Aspekten orientiert ist. Zugleich entäußern sich disziplinspezifische Paradigmen, die mit der Untersuchung und der Quantifizierbarkeit von eben diesem Entscheidungsverhalten einhergehen. Das Akteursmodell eines begrenzt rationalen Akteurs und Annahmen über die Strukturierung von Situationen, die eben diesem ein Entscheiden ermöglichen, zeigen sich hier als wirkmächtig. Verhaltensökonomische Erkenntnisse zur Abweichung von Rationalitätsidealen integrieren psychologische Aspekte der Betrachtung und bilden konstitutive Hintergründe für die Auswahl relevanter Forschungsfragen. Der Erklärwert, welcher dem Einsatz bestimmter Messverfahren zugeschrieben wird, verweist ebenfalls auf disziplinspezifische Wissensbestände der wirtschaftswissenschaftlichen Forschungsdisziplin. Der sprachliche Code der Welt der Ökonomie und der Einsatz monetärer Anreize als (positive und negative) Sanktionen sind Momente dieser Modulation. Gleichsam ist der technisch vermittelte Handlungs- und die Objektivierungszwang Ausdruck eben dieses Mikroökonomie-Moduls. Es werden technisch vermittelte Situationen strategischen Handelns produziert. Zwischen den Optionen oder 
innerhalb des gegebenen Entscheidungsrahmens herrscht Wahlfreiheit für die ProbandInnen, doch allgemein befinden sie sich in einer Situation des Wahl- oder Handlungszwangs.

\section{Spiel(er)-Modul}

Ein zentrales Moment der Rahmenbildung im Laboratop bildet die Ebene des Doings der rationalen und regelkonformen Verhaltenserwartungen. Das System der Konventionen, auf welches diese Ebene Bezug nimmt, wurde als Spiel(er)Modul bezeichnet. Diese Bezeichnung wurde nicht primär gewählt, um darauf hinzuweisen, dass viele der produzierten Handlungssituationen solche des strategischen Handelns von Akteuren sind. Vielmehr zielte dieser Begriff darauf ab, die Spieler und Spielzüge zu charakterisieren, welche das ökonomische Laboratop auszeichnen. Jedes Spiel braucht seine Spieler. Die Spieler in der Welt des ökonomischen Laboratops sind die ProbandInnen und die ExperimentatorInnen. Sie sind situierte Akteure und das Produkt ihres gemeinsamen Handelns in der sozialen Situation des ökonomischen Labors. Ein zentrales Rahmungselement sind die unterschiedlichen Handlungsspielräume, die diesen Spielern zur Verfügung stehen. Die Ethnomethoden und Objektivationen, die sich hier in der empirischen Situation als handlungswirksam erweisen, sind eben solche, die aufzeigen, welche Art regelkonformen Verhaltens von den Handelnden erwartet wird und welche Konsequenzen ein Zuwiderhandeln nach sich zieht. Nicht nur sprachliche und körperliche Formen kommunikativen Handelns erweisen sich hier als relevante Rahmungselemente, sondern auch materielle und technische Objektivationen, die Handlungsspielräume eröffnen und begrenzen. Diese Rahmungselemente treten vor allem auf der Ebene der situativen Realisierung in Erscheinung.

Die ineinander verschachtelte Verknüpfung und Verankerung dieser Moduln führt zur Hervorbringung des Phänomens des ökonomischen Laboratops in seiner typischen Ordnungshaftigkeit. Nachfolgend werden die zentralen Verknüpfungen der Moduln resümiert. Ein konstitutives Element der Spielerrollen und der damit einhergehenden Handlungsspielräume bildet die Verbindung zwischen Spiel(er)-Modul und Experiment-Modul. Mit diesem geht der Anspruch einer standardisierten wissenschaftlichen Untersuchung des Verhaltens der ProbandInnen einher. Den Spielern, in der Rolle der ExperimentatorInnen, werden in dieser sozialen Situation beispielsweise andere Kommunikations- und Bewegungsrechte zuerkannt als den ProbandInnen. Wie die empirischen Beispiele zeigten, setzen die ExperimentatorInnen diese in musterhaften Formen kommunikativen Handelns zur Strukturierung und Kontrolle des Laborgeschehens ein. Das Spiel(er)-Modul bildet das zentrale integrative Moment, welches die rahmeninduzierende Wirkung der Prinzipien der anderen beiden Moduln in der empirischen 
Vollzugswirklichkeit des Laborgeschehens ermöglicht. Räumlich-materielle Rahmungselemente, wie die Trennwände zwischen den Arbeitskabinen oder die Spielchips zur Platzzuweisung, sind Ausdruck der Standardisierungsansprüche des Experiment-Moduls. Als induzierte Rahmungselemente werden sie jedoch erst wirksam, wenn die eintretenden Personen das Spiel(er)-Modul anerkennen, ihre Rolle als ProbandInnen annehmen und dem handlungsstrukturierenden und sinnstiftenden Aufforderungscharakter dieser Objektivationen Folge leisten. Auch im Fall der Instruktionen, welche als zentrales Mittel der Situationsdarstellung betrachtet werden, zeigt sich die Verbindung der drei Moduln. Einerseits sind sie Ausdruck der Standardisierungsansprüche des Experiment-Moduls und bieten auf textlicher Ebene eine gleichartige Informationsgrundlage für die ProbandInnen. Durch ihren sprachlichen Code, die Betonung von Gewinnmöglichkeiten und das gleichzeitige Ausweisen der experimentspezifischen Spielerrollen und Handlungsspielräume verknüpfen sie das Spiel(er)-Modul mit dem Mikroökonomie-Modul. Diese Art der textlichen Darstellung der Spielsituation zielt darauf ab, die von den Forschenden als relevant antizipierten Rahmungselemente des jeweiligen Experiments zu explizieren. Um die Chance einer Übernahme dieser Rahmungsrationalitäten durch die ProbandInnen zu erhöhen, werden unterschiedliche kommunikative Methoden eingesetzt, wie Tests, Beispielerörterungen oder der Rückverweis auf die Instruktionen bei der Beantwortung von Nachfragen. Die Datenerhebung, welche die Grundlage für die wissenschaftliche Analyse bildet, erfolgt durch das Interagieren der ProbandInnen mit Bildschirmoberflächen, welche mit Hilfe der Eingabegeräte (Tastatur und Maus) realisiert werden muss. Auch hier basieren die Darstellungen der Spielsituationen primär auf textlichen Elementen. Die für die Instruktionen dargestellte Verbindung von Experiment- und Mikroökonomie-Modul wirkt hier gleichsam. Die technische Mediation kommunikativer Handlungen verschiebt die Handlungsstrukturierung und -begrenzung des Spiel(er)-Moduls hier jedoch maßgeblich auf die technische Ebene. Es sind nur Eingaben möglich, die auch dem vorgesehenen Handlungsspielraum und den Maßgaben der Objektivierung kommunikativen Handelns entsprechen. In der Konsequenz sind auch die Möglichkeiten einer Überschreitung des Handlungsspielraums technisch begrenzt. Innerhalb des gegebenen Entscheidungsrahmens besteht Wahlfreiheit für die ProbandInnen. Zugleich bedingt die sequenzielle Organisation des Ablaufs einen Handlungszwang für die ProbandInnen, der eine Eingabe notwendig macht. Dies regt zu einer Fokussierung auf die induzierten Rahmungselemente an und ermöglicht es aus Sicht der Forschenden zugleich, die Handlungen der ProbandInnen als Entscheidung zu deuten. Einen zentralen Anreiz für die Entwicklung von Strategien und ein bewusstes Wählen einzelner Handlungsmöglichkeiten bildet der in Aussicht gestellte Gewinn 
am Ende des Experiments. Diese Bezugnahme auf die monetäre Dimension des Mikroökonomie-Modul bestärkt zugleich die Wirkung des Spiel(er)-Moduls während des gesamten Laborgeschehens. Als positive Sanktion wird spielkonformes Verhalten während der Durchführung der Experimente belohnt. Als negative Sanktion für eine Missachtung der Spielregeln wird jedoch mit dem Verlust der erspielten Gewinne gedroht.

Die drei in der Analyse herausgestellten Moduln und die charakteristischen Formen ihrer Entäußerung im Vollzug des Laborgeschehens bilden zusammen die „Modulation des ökonomischen Laboratops“. Das Spiel(er)-Modul bildet hier das zentrale Verbindungsmoment, welches die Realisierung des Experiment- und des Mikroökonomie-Moduls in der sozialen Situation des Experiments bestärkt und ermöglicht.

Das ökonomische Laboratop als Entrückung vom Alltag beansprucht seine Geltung innerhalb der zeitlichen und räumlichen Klammer der Durchführung des Experiments. Wie bei der Betrachtung der Prä- und Postsequenzen und des Rekrutierungsprozesses ausgewiesen wurde, gibt es jedoch zugleich ,lose Enden', an denen bestimmte Rahmungsaspekte durch vorgängige oder nachfolgende Situationen beeinflusst werden.

Der Werbeslogan der wirtschaftswissenschaftlichen Experimente ,Spielend Geld verdienen“ erweist sich im Anschluss an diese Ausführungen als bezeichnend für das untersuchte Kommunikationsphänomen. Dies jedoch nicht aus dem Grund, dass es im ökonomischen Laboratop ,einfach“ ist, Geld zu verdienen. Vielmehr gilt es im Rahmen dieser Modulation, den Spielkosmos und seine Regel anzuerkennen, wenn man Teil des Spiels sein möchte. Die ProbandInnen entscheiden sich freiwillig dazu, Teil eben dieses Spiels zu sein. Viele von ihnen sind sogar häufige Besucher in der von mir untersuchten Spielwelt des ökonomischen Laboratops. 
Open Access Dieses Kapitel wird unter der Creative Commons Namensnennung 4.0 International Lizenz (http://creativecommons.org/licenses/by/4.0/deed.de) veröffentlicht, welche die Nutzung, Vervielfältigung, Bearbeitung, Verbreitung und Wiedergabe in jeglichem Medium und Format erlaubt, sofern Sie den/die ursprünglichen Autor(en) und die Quelle ordnungsgemäß nennen, einen Link zur Creative Commons Lizenz beifügen und angeben, ob Änderungen vorgenommen wurden.

Die in diesem Kapitel enthaltenen Bilder und sonstiges Drittmaterial unterliegen ebenfalls der genannten Creative Commons Lizenz, sofern sich aus der Abbildungslegende nichts anderes ergibt. Sofern das betreffende Material nicht unter der genannten Creative Commons Lizenz steht und die betreffende Handlung nicht nach gesetzlichen Vorschriften erlaubt ist, ist für die oben aufgeführten Weiterverwendungen des Materials die Einwilligung des jeweiligen Rechteinhabers einzuholen. 